Supporting Information for:

\title{
Synthesis and Magnetism of Neutral, Linear Metallocene Complexes of Terbium(II) and Dysprosium(II)
}

Colin A. Gould, ${ }^{\dagger}{ }^{\S}$ K. Randall McClain, ${ }^{\star \S}$ Jason M. Yu, ${ }^{\mathbb{I}}$ Thomas J. Groshens, ${ }^{\ddagger}$ Filipp Furche, ${ }^{\mathbb{I} *}$ Benjamin G. Harvey, ${ }^{\dagger *}$ and Jeffrey R. Long ${ }^{\dagger \|_{*}}$

$\dagger$ U.S. Navy, Naval Air Warfare Center, Weapons Division, Research Department, Chemistry Division, China Lake, California, 93555, United States

tDepartment of Chemistry and "Department of Chemical and Biomolecular Engineering, University of California, Berkeley, Berkeley, California 94720, United States

${ }^{I}$ Department of Chemistry, University of California, Irvine, 1102 Natural Sciences II, Irvine California 92697-2025, United States

${ }^{\perp}$ Materials Sciences Division, Lawrence Berkeley National Laboratory, Berkeley, California 94720, United States 


\section{Table of Contents}

$\begin{array}{ll}\text { Synthesis of 1-3 } & \text { S3 }\end{array}$

Infrared Spectroscopy $\quad$ S5

UV-vis-NIR Spectroscopy $\quad$ S7

X-ray Crystallography Data Collection and Refinement Details $\quad$ S11

Magnetic Measurements $\quad$ S16

$\begin{array}{ll}\text { Dc Magnetic Susceptibility Measurements } & \text { S16 }\end{array}$

Ac Magnetic Susceptibility Measurements and Cole-Cole Plots $\quad$ S20

Dc Magnetic Relaxation Measurements $\quad$ S40

Analysis of Magnetic Relaxation Dynamics $\quad$ S55

Magnetic Hysteresis Measurements $\quad$ S63

$\begin{array}{ll}\text { DFT Calculations } & \text { S70 }\end{array}$

$\begin{array}{ll}\text { References } & \text { S86 }\end{array}$ 
General Procedures. All manipulations were performed using Schlenk or glovebox techniques under an atmosphere of purified argon with rigorous exclusion of water and oxygen. All solvents were purchased from Sigma-Aldrich as anhydrous grade in Sure/Seal ${ }^{\mathrm{TM}}$ bottles, purged for several hours with purified argon and stored over activated $3 \AA$ molecular sieves in an argon filled glovebox. Celite (AW Standard Super-Cel ${ }^{\circledR}$ NF) was purchased from Sigma-Aldrich and dried under vacuum at $150-200{ }^{\circ} \mathrm{C}$ overnight before being transferred to the glovebox. $\mathrm{KC}_{8}$ was purchased from Strem and used as received. Anhydrous $\mathrm{LnI}_{3}(\mathrm{Ln}=\mathrm{Dy}, \mathrm{Tb})$ were purchased from Alfa Aesar as Ultra Dry ${ }^{\mathrm{TM}}$ grade reagents and used as received. The halide abstraction reagent, $\left[\mathrm{H}\left(\mathrm{SiEt}_{3}\right)_{2}\right]\left[\mathrm{B}\left(\mathrm{C}_{6} \mathrm{~F}_{5}\right)_{4}\right]$, was prepared freshly before use via a reported method. ${ }^{1}$ The salt $\mathrm{NaCp}{ }^{i \mathrm{Pr} 5}$ was prepared via a previously published method. ${ }^{2}$ NMR spectra were recorded on a Bruker Avance $300 \mathrm{MHz}$ or $500 \mathrm{MHz}$ spectrometer and internally referenced to the residual solvent signals. FTIR spectra were recorded on a Perkin Elmer Avatar Spectrum 400 FTIR Spectrometer equipped with an attenuated total reflectance (ATR) attachment. UV-vis-NIR absorption spectra were collected with a CARY 5000 spectrophotometer interfaced with Varian WinUV software. Matrix Assisted Laser Desorption Ionization Time of Flight (MALDI-TOF) mass spectra were recorded on an Applied Biosystems Voyager-DE PRO Workstation in positive ion mode. Samples were cocrystalized in an anthracene matrix on an AB SCIEX MALDI-TOF stainless steel sample plate. Spectra were averaged over 200 laser pulses with a low mass gate of 300 Dalton and a high mass gate of 2000 Dalton. Elemental analyses $(\mathrm{C}, \mathrm{H}, \mathrm{N})$ were performed by the Microanalytical Facility at the University of California, Berkeley using a Perkin-Elmer 2400 Series II combustion analyzer. Magnetic susceptibility measurements were collected using a Quantum Design MPMS2 SQUID magnetometer.

Synthesis of $\left[\mathbf{T b}\left(\mathbf{C p}^{i \mathrm{Pr}^{5}}\right)_{2}\right]\left[\mathbf{B}\left(\mathbf{C}_{6} \mathbf{F}_{5}\right)_{4}\right](\mathbf{1})$. Under argon, $\mathrm{TbI}_{3}(0.250 \mathrm{~g} / 0.463 \mathrm{mmol})$ and $\mathrm{NaCp}^{i \mathrm{Pr} 5}$ $(0.350 \mathrm{~g} / 1.17 \mathrm{mmol})$ were combined in a $35 \mathrm{~mL}$ pressure flask containing a glass-coated magnetic stirring bar and toluene $(15 \mathrm{~mL})$ was added. The reaction flask was covered in $\mathrm{Al}$ foil, and heated gradually to $160^{\circ} \mathrm{C}$ with vigorous stirring. After $72 \mathrm{~h}$ at $160{ }^{\circ} \mathrm{C}$, the flask was allowed to cool to room temperature, the reaction mixture was filtered through Celite and the pad washed with additional toluene $(3 \times 5 \mathrm{~mL})$. Solvent was removed from the filtrate under vacuum and the residue was gently heated $\left(50{ }^{\circ} \mathrm{C}\right)$ under vacuum for $1 \mathrm{~h}$. The residue was taken up in pentane $(25 \mathrm{~mL})$, stirred for $0.5 \mathrm{~h}$, filtered through Celite and the pad washed with additional pentane ( $3 \mathrm{X} 5 \mathrm{~mL}$ ). Solvent was removed from the filtrate under vacuum and the residue was gently heated $\left(50{ }^{\circ} \mathrm{C}\right)$ under vacuum for $0.5 \mathrm{~h}$ to give crude $\mathrm{Tb}\left(\mathrm{Cp}^{i \mathrm{Pr} 5}\right)_{2} \mathrm{I}$ as a yellow powder. This was dissolved with benzene $(10 \mathrm{~mL})$ in a $25 \mathrm{~mL}$ Schlenk flask equipped with a Teflon magnetic stirring bar to give a yellow solution. Under vigorous stirring at room temperature, a solution of $\left[\mathrm{H}\left(\mathrm{SiEt}_{3}\right)_{2}\right]\left[\mathrm{B}\left(\mathrm{C}_{6} \mathrm{~F}_{5}\right)_{4}\right]$ $(0.342 \mathrm{~g} / 0.375 \mathrm{mmol})$ in benzene $(5 \mathrm{~mL})$ was added dropwise to the $\mathrm{Tb}\left(\mathrm{Cp}^{\mathrm{iPr} 5}\right)_{2} \mathrm{I}$ solution over 5 min. After stirring for $72 \mathrm{~h}$ at room temperature, solvent was removed from the reaction mixture under vacuum, the residue was slurried with pentane $(20 \mathrm{~mL})$ for $0.5 \mathrm{~h}$ and isolated as an orange powder on a medium-porosity fritted filter after washing with pentane $(3 \mathrm{X} 10 \mathrm{~mL})$. This was dried under vacuum, dissolved in dichloromethane $(4 \mathrm{~mL})$, filtered with aid of Celite through a $0.2 \mu \mathrm{m}$ porosity PTFE syringe filter and layered with pentane $(15 \mathrm{~mL})$ in a $20 \mathrm{~mL}$ vial. After $48 \mathrm{~h}$ at 25 ${ }^{\circ} \mathrm{C}$ bright orange needle crystals were obtained. These were isolated on a medium-porosity fritted filter, washed with pentane $(5 \mathrm{~mL})$, benzene $(5 \mathrm{~mL})$ and pentane $(5 \mathrm{~mL})$. The crystallization was repeated and the crystals dried under vacuum to yield 1 as orange needles $(0.330 \mathrm{~g} / 0.238 \mathrm{mmol} /$ $51 \%$ based on $\mathrm{TbI}_{3}$ ). $\mathrm{C}_{64} \mathrm{H}_{70} \mathrm{BTbF}_{20}$ (1388.97): calcd (\%) C 55.34, $\mathrm{H} 5.08$; found (\%) C 55.18, $\mathrm{H}$ 5.00 . 
Synthesis of $\left[\mathbf{T b}\left(\mathbf{C p}^{i \mathrm{Pr} 5}\right)_{2}\right]$ (2). Under argon, $\mathrm{TbI}_{3}(0.361 \mathrm{~g} / 0.670 \mathrm{mmol})$ and $\mathrm{NaCp}^{i \mathrm{Pr} 5}(0.50 \mathrm{~g} /$ $1.67 \mathrm{mmol}$ ) were combined in a $35 \mathrm{~mL}$ pressure flask containing a glass-coated magnetic stirring bar and toluene $(20 \mathrm{~mL})$ was added. The reaction flask was covered in $\mathrm{Al}$ foil, and heated gradually to $160{ }^{\circ} \mathrm{C}$ with vigorous stirring. After $72 \mathrm{~h}$ at $160{ }^{\circ} \mathrm{C}$, the flask was allowed to cool to room temperature, the reaction mixture was filtered through Celite and the pad washed with additional toluene ( $3 \mathrm{X} 5 \mathrm{~mL})$. Solvent was removed from the filtrate under vacuum and the residue was gently heated $\left(50^{\circ} \mathrm{C}\right)$ under vacuum for $1 \mathrm{~h}$. The residue was taken up in pentane $(25 \mathrm{~mL})$, stirred for $0.5 \mathrm{~h}$, filtered through Celite and the pad washed with additional pentane ( $3 \mathrm{X} 5 \mathrm{~mL})$. Solvent was removed from the filtrate under vacuum and the residue was gently heated $\left(50{ }^{\circ} \mathrm{C}\right)$ under vacuum for $0.5 \mathrm{~h}$ to give crude $\mathrm{Tb}\left(\mathrm{Cp}^{i \mathrm{Pr} 5}\right)_{2} \mathrm{I}$ as a yellow powder. Under argon, the crude $\mathrm{Tb}\left(\mathrm{Cp}^{\mathrm{iPr} 5}\right)_{2} \mathrm{I}$ was placed in a $50 \mathrm{~mL}$ Schlenk flask and dissolved with benzene $(15 \mathrm{~mL})$ to give a yellow solution, $\mathrm{KC}_{8}(0.180 \mathrm{~g} / 1.34 \mathrm{mmol})$ was added and the flask was covered in $\mathrm{Al}$ foil and allowed to stir at $25{ }^{\circ} \mathrm{C}$ for 6 days (reaction solution gradually changed from yellow to vibrant orange and bronze $\mathrm{KC}_{8}$ slowly converted to black graphite). Solvent was removed under vacuum, the residue was extracted by stirring with hexane $(25 \mathrm{~mL})$ for $30 \mathrm{~min}$., filtered through Celite and the pad washed with additional hexane $(3 \times 5 \mathrm{~mL})$ to give a vibrant orange filtrate. The solution was concentrated in a $40 \mathrm{~mL}$ vial under vacuum until copious precipitation of orange/amber solid $(\sim 10 \mathrm{~mL})$; this was re-dissolved by heating the solution to boiling. The vial was then wrapped in $\mathrm{Al}$ foil and allowed to cool slowly to $25^{\circ} \mathrm{C}$, after several days, orange amber crystals appeared and the vial was then left at $-35{ }^{\circ} \mathrm{C}$ to complete the crystallization. Orange/amber prisms of 2 were isolated in two crops, washed with cold pentane $(2 \mathrm{X} 2 \mathrm{~mL})$ and dried under vacuum $(0.194 \mathrm{~g} \mathrm{/}$ $0.273 \mathrm{mmol} / 41 \%$ based on $\mathrm{TbI}_{3}$ ). MALDI ToF MS m/z: 709.2 ([M] $\left.{ }^{+}\right) . \mathrm{C}_{40} \mathrm{H}_{70} \mathrm{~Tb}$ (709.93): calcd (\%) C 67.67, H 9.94; found (\%) C 67.53, H 9.68.

Synthesis of [Dy(C $\left.\left.\mathbf{C p}^{i \mathrm{Pr} 5}\right)_{2}\right]$ (3). Following the general procedure used for synthesis of $\mathbf{2}, \mathbf{3}$ was synthesized from $\mathrm{DyI}_{3}(0.364 \mathrm{~g} / 0.670 \mathrm{mmol}), \mathrm{NaCp}^{\mathrm{iPr} 5}(0.50 \mathrm{~g} / 1.67 \mathrm{mmol})$ and $\mathrm{KC}_{8}(0.180 \mathrm{~g} /$ $1.34 \mathrm{mmol})$. The crude product was recrystallized from hexane using the same method outlined for 2, yielding orange/amber prisms in two crops $\left(0.231 \mathrm{~g} / 0.324 \mathrm{mmol} / 48 \%\right.$ based on $\left.\mathrm{DyI}_{3}\right)$. MALDI ToF MS m/z: 713.4 ([M] $]^{+}$). $\mathrm{C}_{40} \mathrm{H}_{70}$ Dy (713.50): calcd (\%) C 67.34, H 9.89; found (\%) C 67.20, H 9.47. 


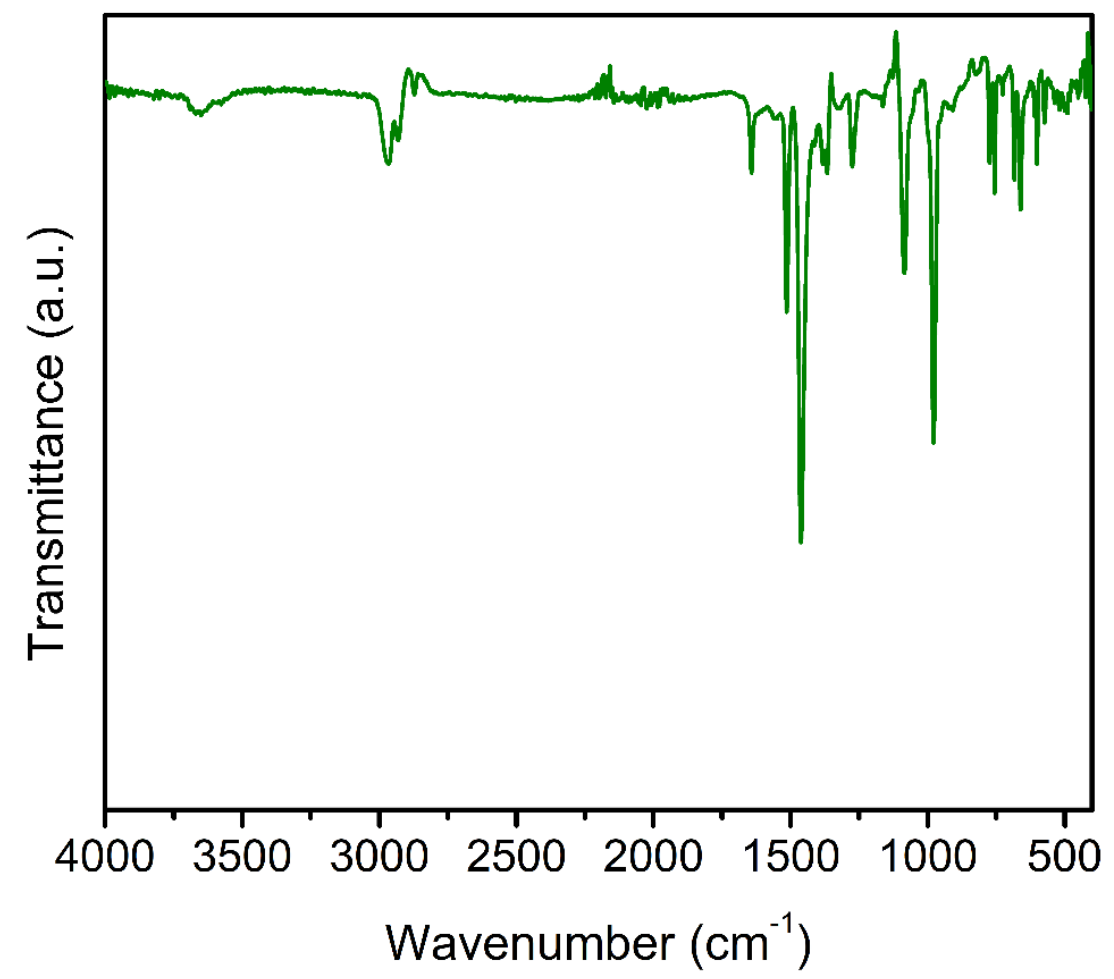

Figure S1. Infrared spectrum of $\mathbf{1}$.

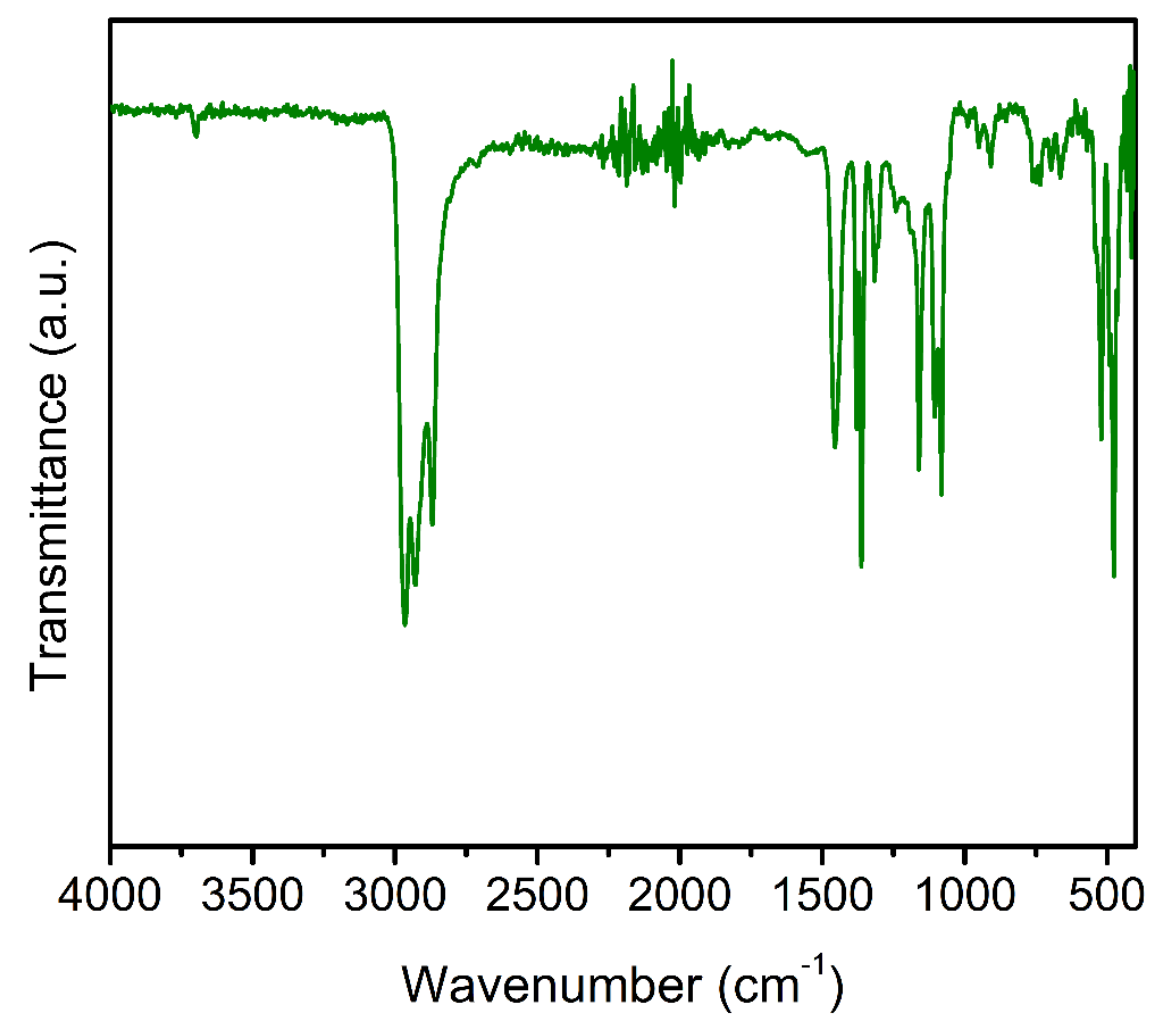

Figure S2. Infrared spectrum of 2. 


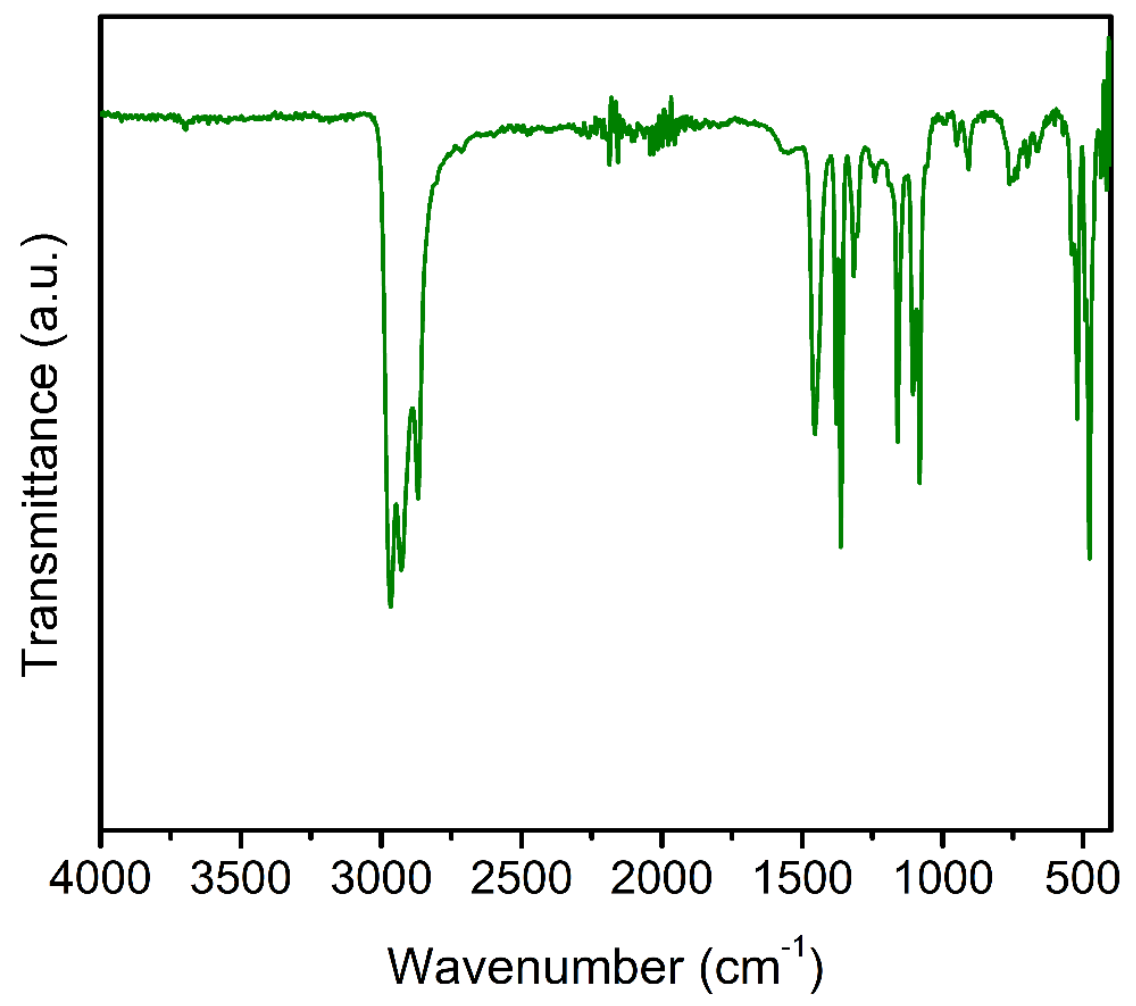

Figure S3. Infrared spectrum of 3 . 
UV-vis-NIR Spectroscopy. UV-Vis-NIR spectroscopy was performed on solutions of $\mathbf{2}$ and $\mathbf{3}$ in hexanes. Two stock solutions were made for each sample (to avoid mass error), $0.46 \mathrm{mM}$ and $0.53 \mathrm{mM}$ for 2 and 0.48 and $0.58 \mathrm{mM}$ for 3 . A total of seven measurements were made for each sample, four with serial dilutions of the first stock solution and three with serial dilutions of the second stock solution. Plots of concentration versus absorbance were used to extract extinction coefficients for each of the features observed in the spectrum.

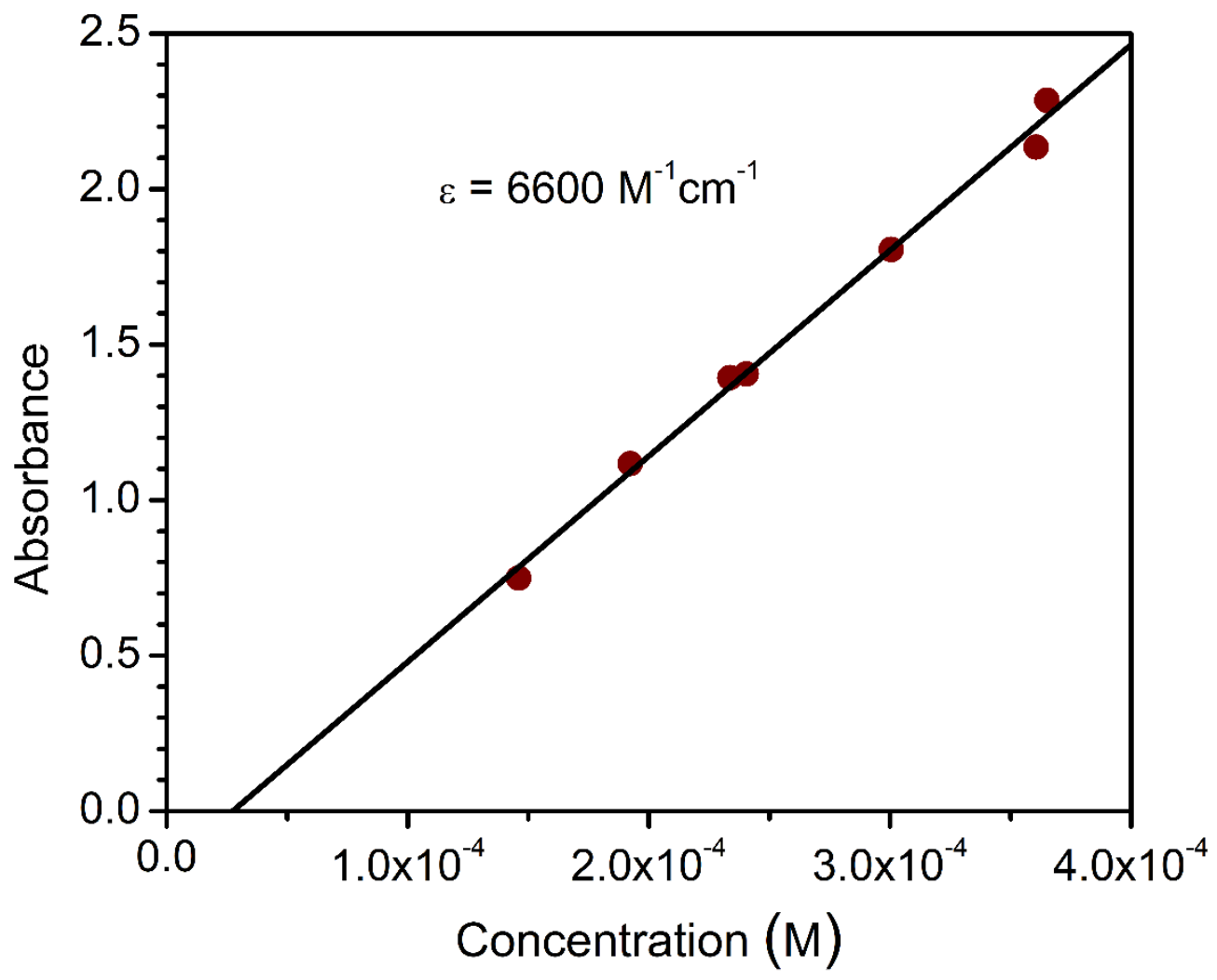

Figure S4. Plot of absorbance versus concentration for the feature at $303 \mathrm{~nm}$ in the UV-Vis-NIR spectrum of 2. Maroon points represent experimental data and the black line represents the fit to the data used to extract the extinction coefficient. 


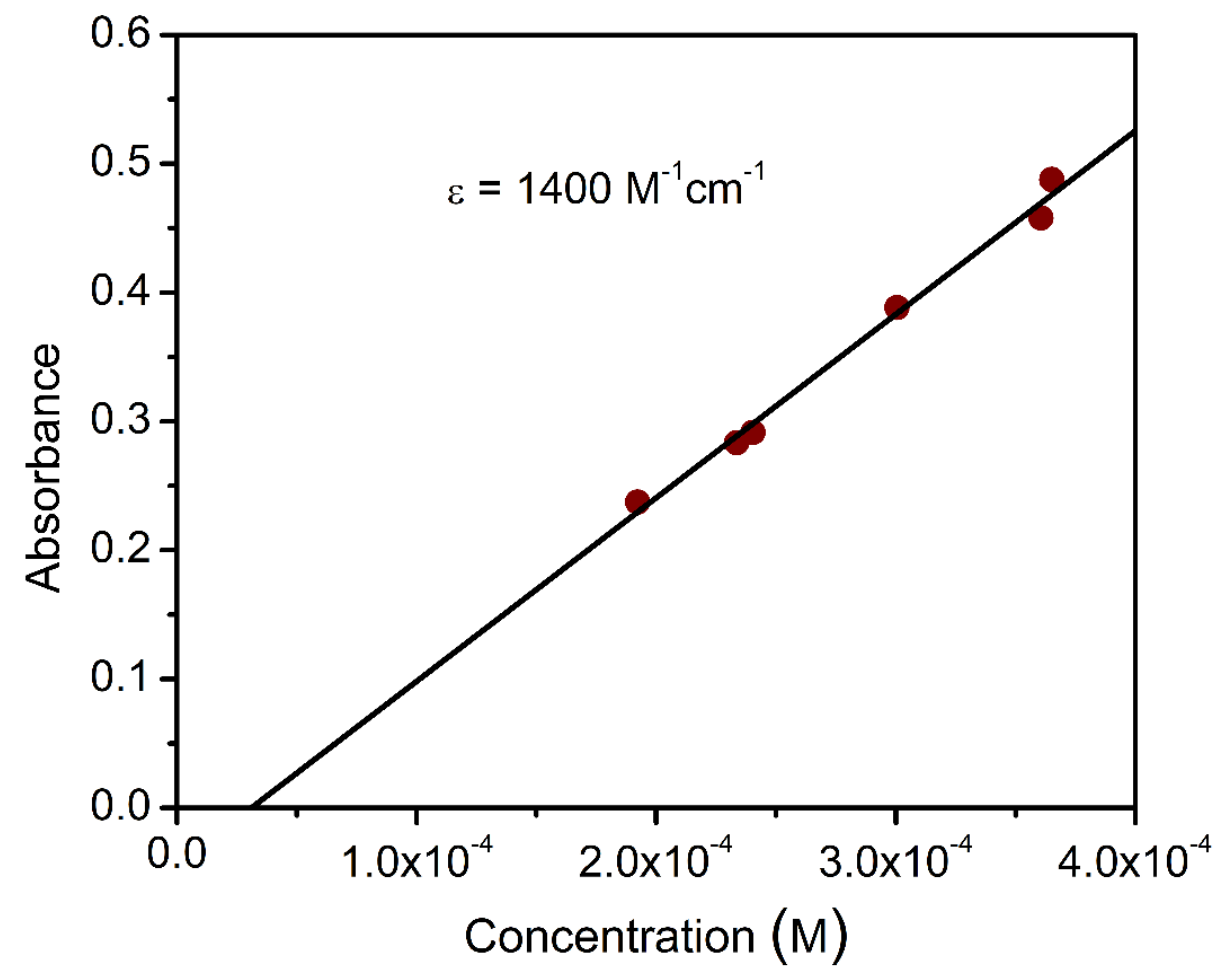

Figure S5. Plot of absorbance versus concentration for the feature at $429 \mathrm{~nm}$ in the UV-Vis-NIR spectrum of 2. Maroon points represent experimental data and the black line represents the fit to the data used to extract the extinction coefficient.

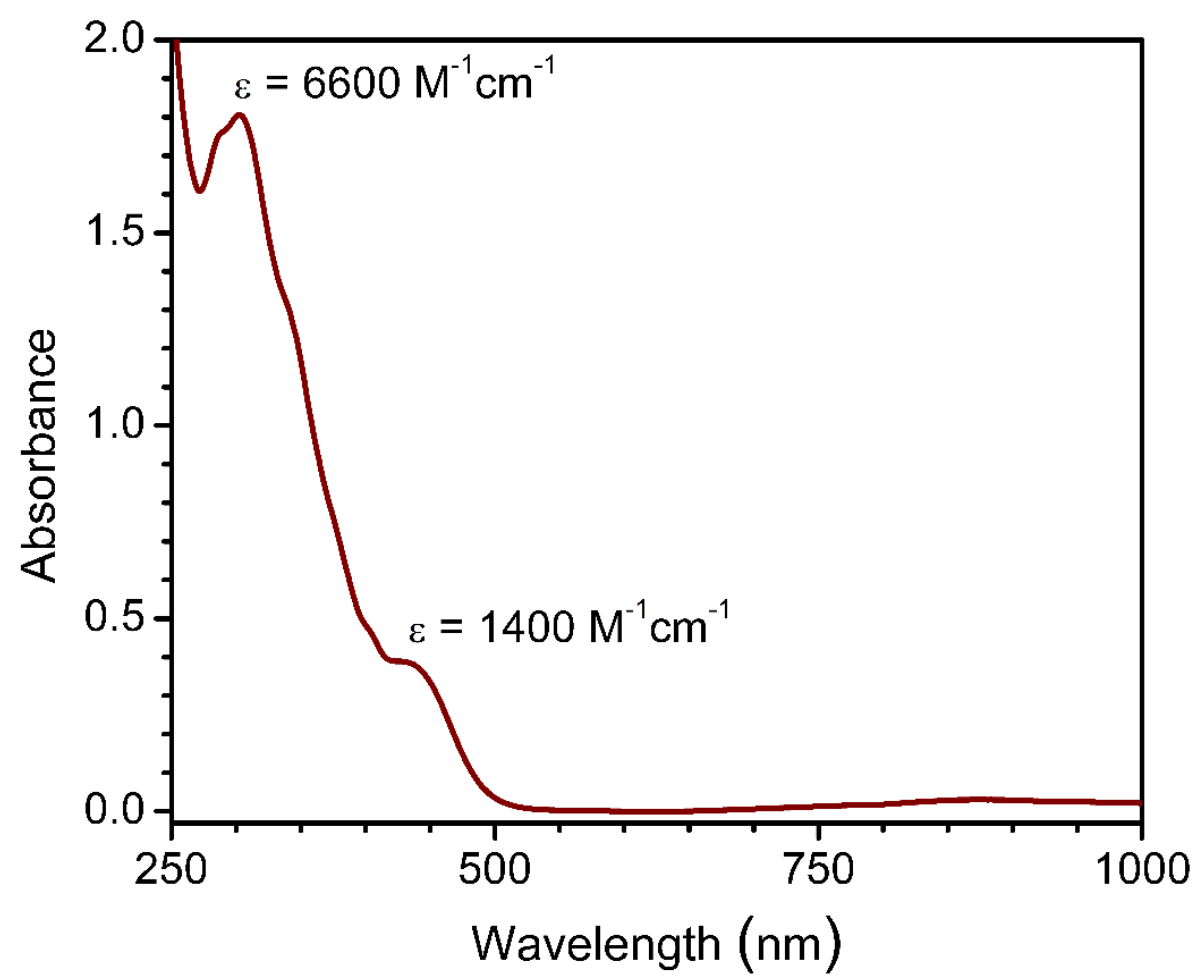

Figure S6. UV-Vis-NIR spectrum of 2. 


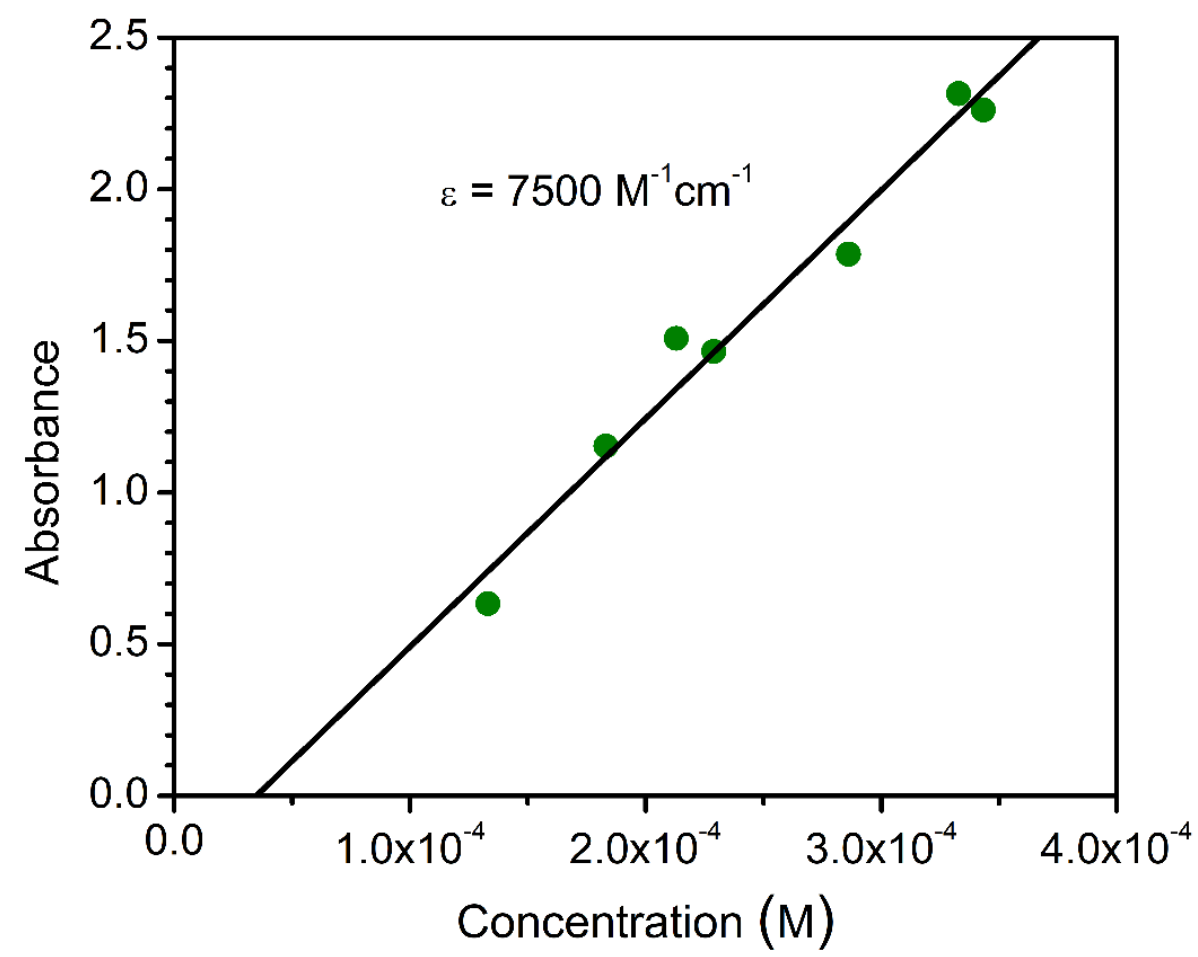

Figure S7. Plot of absorbance versus concentration for the feature at $298 \mathrm{~nm}$ in the UV-Vis-NIR spectrum of 3. Green points represent experimental data and the black line represents the fit to the data used to extract the extinction coefficient.

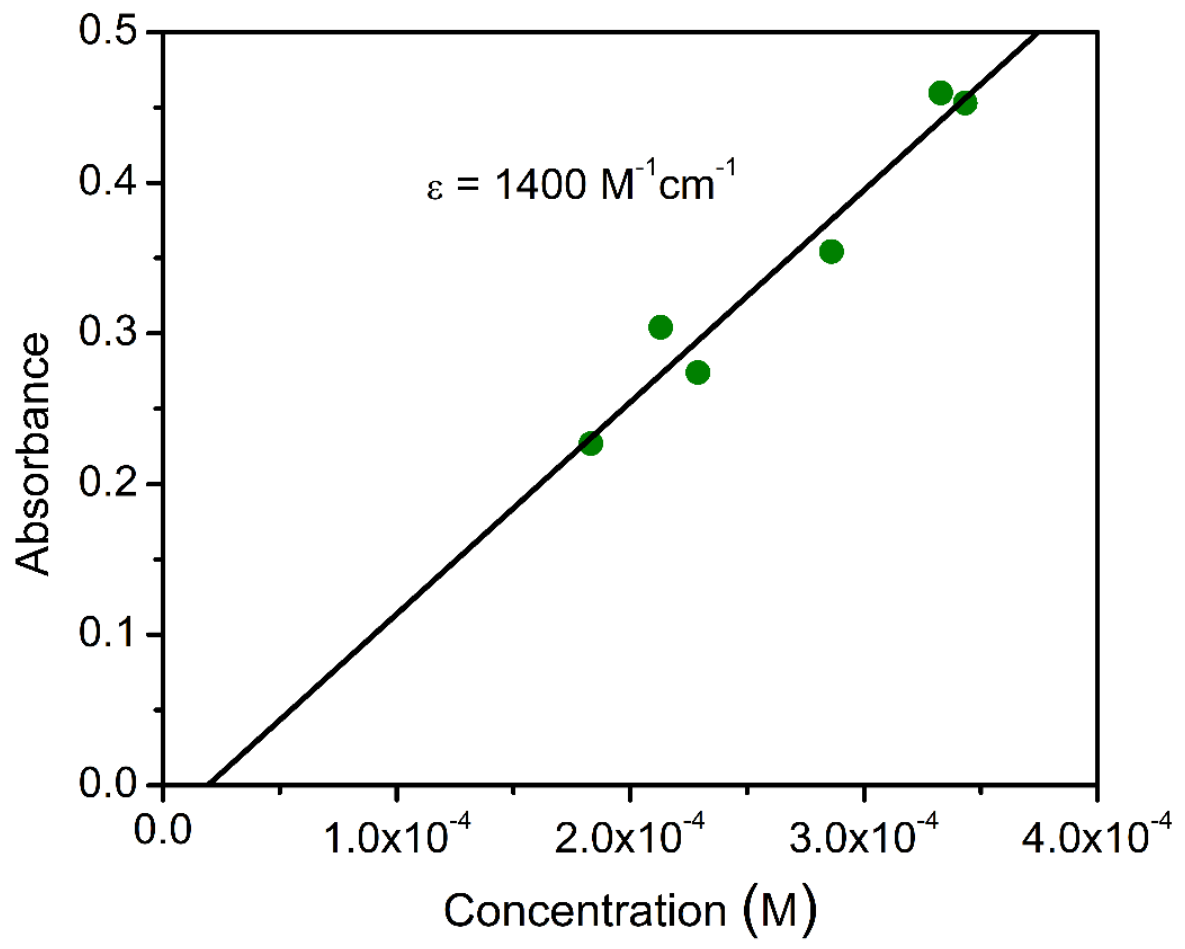

Figure S8. Plot of absorbance versus concentration for the feature at $428 \mathrm{~nm}$ in the UV-Vis-NIR spectrum of 3. Green points represent experimental data and the black line represents the fit to the data used to extract the extinction coefficient. 


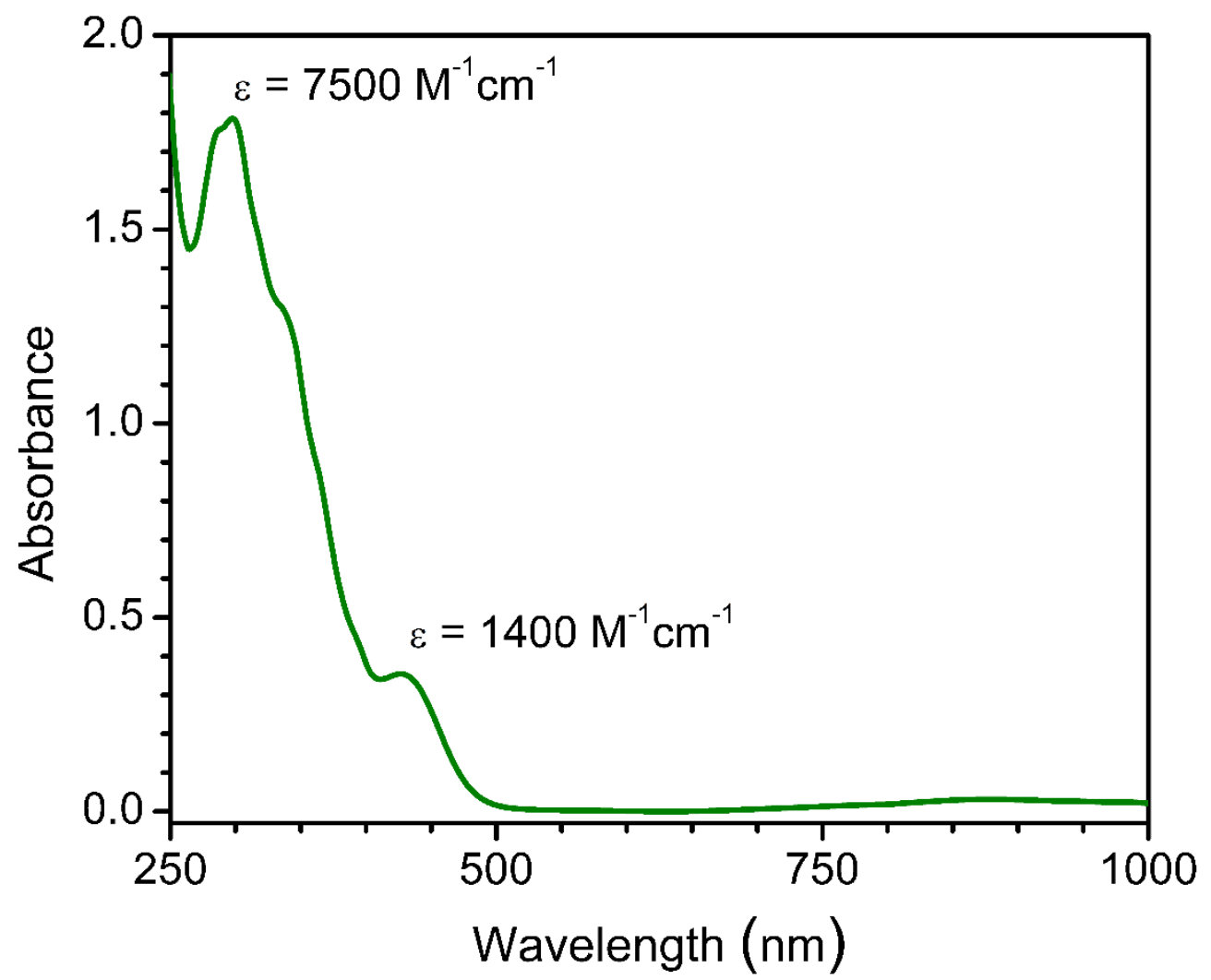

Figure S9. UV-Vis-NIR spectrum of 3. 


\section{X-ray Crystallography Data Collection and Refinement Details}

Single crystal X-ray diffraction data of $\mathbf{1}$ and $\mathbf{2}$ were collected as follows. The sample was coated with Parabar oil and mounted on a MiTeGen polyimide loop in a nitrogen filled glovebox. The X-ray intensity data were measured using a Bruker SMART Apex II diffractometer at $100 \mathrm{~K}$ under a N2 stream of an Oxford Cryostems Cryostream with MoK $\alpha$ radiation (graphite monochrometer). ${ }^{3}$ Data was collected from four $180^{\circ} \omega$ scans for $\mathbf{1}$ and seven scans for $\mathbf{2}$ with $0.5^{\circ}$ steps at $90^{\circ}$ rotation intervals about $\varphi$ and a CCD detector distance of $5 \mathrm{~cm}$. The frames were integrated with the Bruker SAINT Software package using a narrow-frame algorithm. ${ }^{4}$

Single crystals of $\mathbf{3}$ were coated in Paratone-N-oil and mounted on a Kaptan loop. X-ray diffraction data were collected at UC, Berkeley using a Rigaku XtaLAB p200 equipped with a MicroMax-007 HF microfocus rotating anode and a Pilatus 200K hybrid pixel array detector at $100 \mathrm{~K}$ under a N2 stream of an Oxford Cryostems Cryostream with MoK $\alpha$ radiation (graphite monochrometer). The frames were integrated with CrysAlis ${ }^{\text {Pro }}$ software, including a multi-scan absorption correction that was applied using the SCALE3 ABSPACK scaling algorithm within CrysAlis ${ }^{\text {Pro } 5}$. Initial structure solutions were determined using direct methods (SHELXT) and refinements were carried out using SHELXL-2014. ${ }^{6-7}$

$\left[\mathbf{T b}\left(\mathbf{C} \mathbf{p}^{i \mathbf{P r 5}}\right)_{2}\right]\left[\mathbf{B}\left(\mathbf{C}_{6} \mathbf{F}_{5}\right)_{4}\right]$ (1). The compound crystallized in the space group $P 2_{1} / n$ with one molecule in the asymmetric unit. As in the structure of $\left[\mathrm{Dy}\left(\mathrm{Cp}^{i \mathrm{Pr} 5}\right)_{2}\right]\left[\mathrm{B}\left(\mathrm{C}_{6} \mathrm{~F}_{5}\right)_{4}\right]$, anisotropic refinement of the $\mathrm{Tb}$ atom resulted in large thermal displacement parameters, suggesting positional disorder. Reasonable thermal displacement parameters were achieved by modeling the Tb atom over four positions. The sum of the four Tb positions was constrained to equal an occupancy of one using the SUMP command, however competitive refinement did not converge satisfactorily to an integer value. Instead, occupancies were fixed to values close to those obtained by competitive refinement (rounded to two significant figures), resulting in a ratio of 0.36:0.10:0.21:0.33. This disorder may indicate that the $\mathrm{Tb}$ atom can move freely between the two $\mathrm{Cp}^{\mathrm{iPr} 5}$ rings. The ten isopropyl groups of the $\mathrm{Cp}^{\mathrm{iPr} 5}$ rings also showed positional disorder and were each modeled over two positions. The refinement was stabilized through application of 1,2 $(\mathrm{CH}-$ $\left.\mathrm{CH}_{3}\right)$ and 1,3 $\left(\mathrm{CH}_{3} \cdots \mathrm{CH}_{3}\right)$ distance restraints, as well as enhanced rigid bond restraints. In addition, $\mathrm{C}_{\mathrm{Cp}}-\mathrm{C}_{\mathrm{Cp}}-\mathrm{C}(\mathrm{H})$ angles were restrained to be the same. Two of the isopropyl methyl groups could not be stabilized in the refinement unless identical anisotropic displacement parameters were used for them, which were determined by the other methyl carbon in each isopropyl group. All non-H atoms were refined anisotropically.

[Tb(Cp$\left.\left.{ }^{i \operatorname{Pr} 5}\right)_{2}\right]$ (2). The compound crystallized in the space group $P 2{ }_{1} / n$ with half a molecule in the asymmetric unit. All non-H atoms were refined anisotropically.

[Dy $\left.\left(\mathbf{C p}^{i \mathbf{P r 5}}\right)_{2}\right]$ (3). The compound crystallized in the space group $P 2{ }_{1} / n$ with half a molecule in the asymmetric unit. All non-H atoms were refined anisotropically. 
Table S1. Structure details for 1-3.
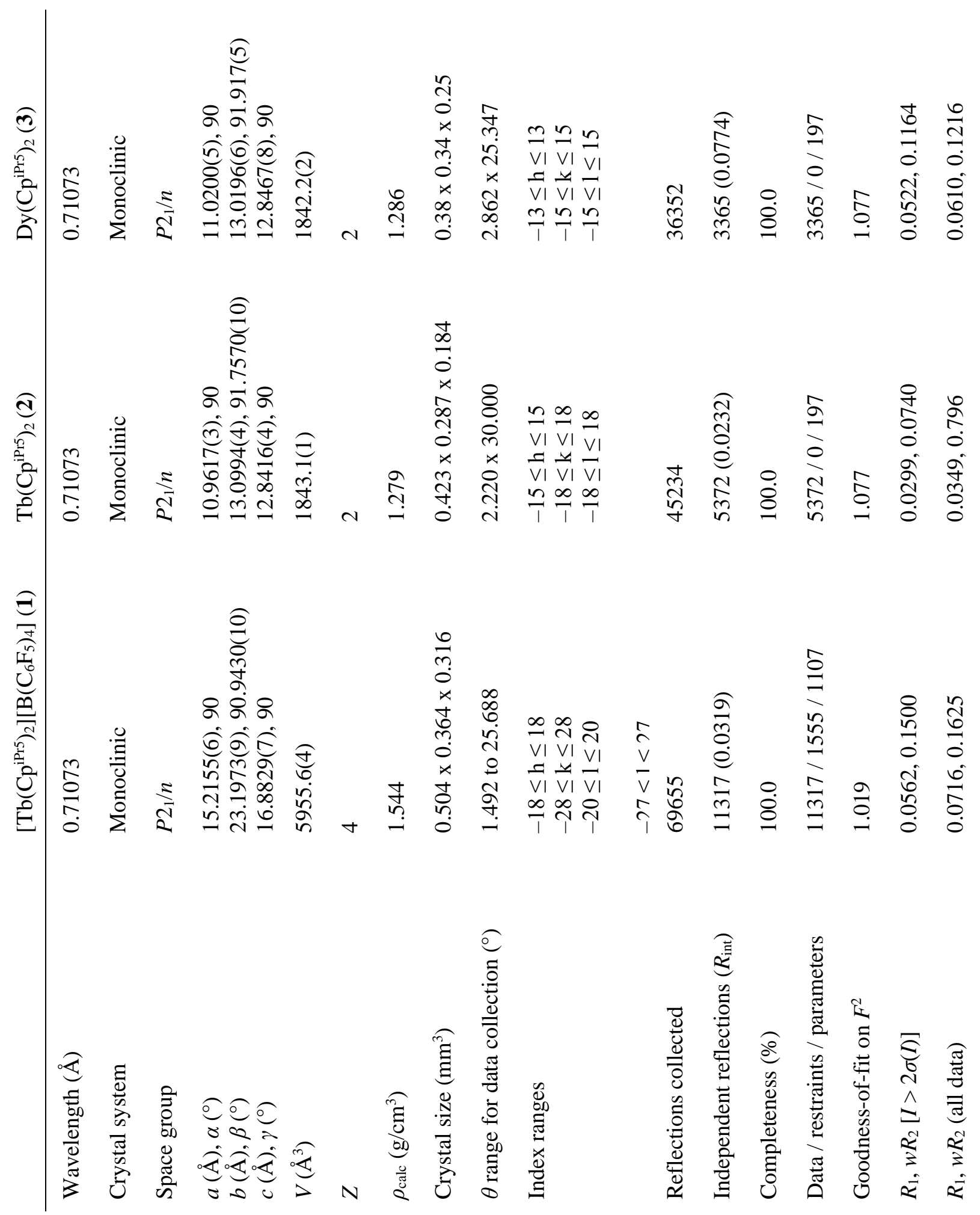


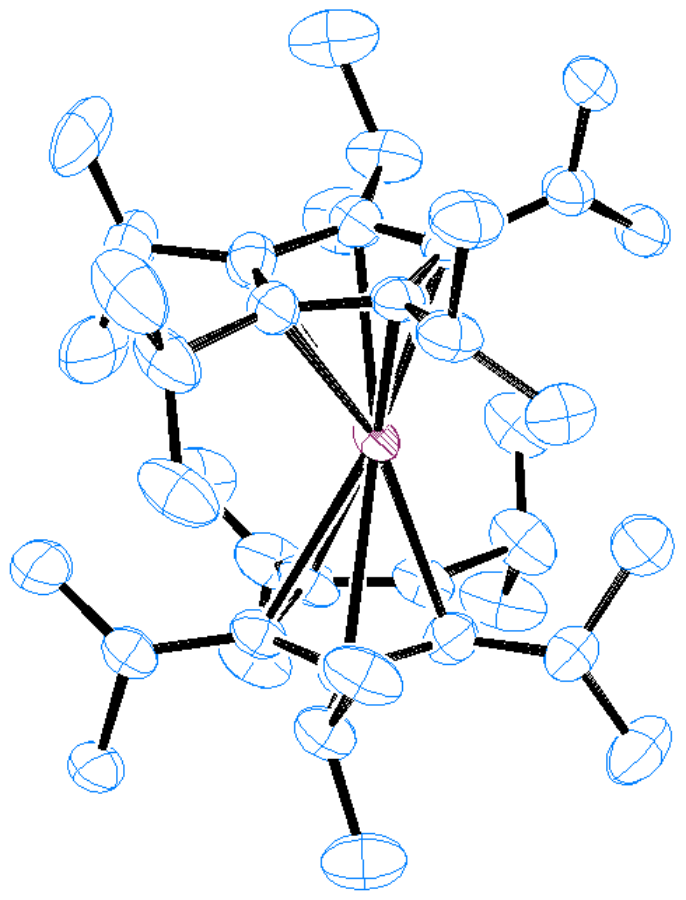

Figure S10. Solid-state structure of the $\left[\mathrm{Tb}\left(\mathrm{Cp}^{i \mathrm{Pr} 5}\right)_{2}\right]^{+}$cation in 1 with thermal ellipsoids at the $30 \%$ probability level. Hydrogen atoms and the disordered component are omitted for clarity.

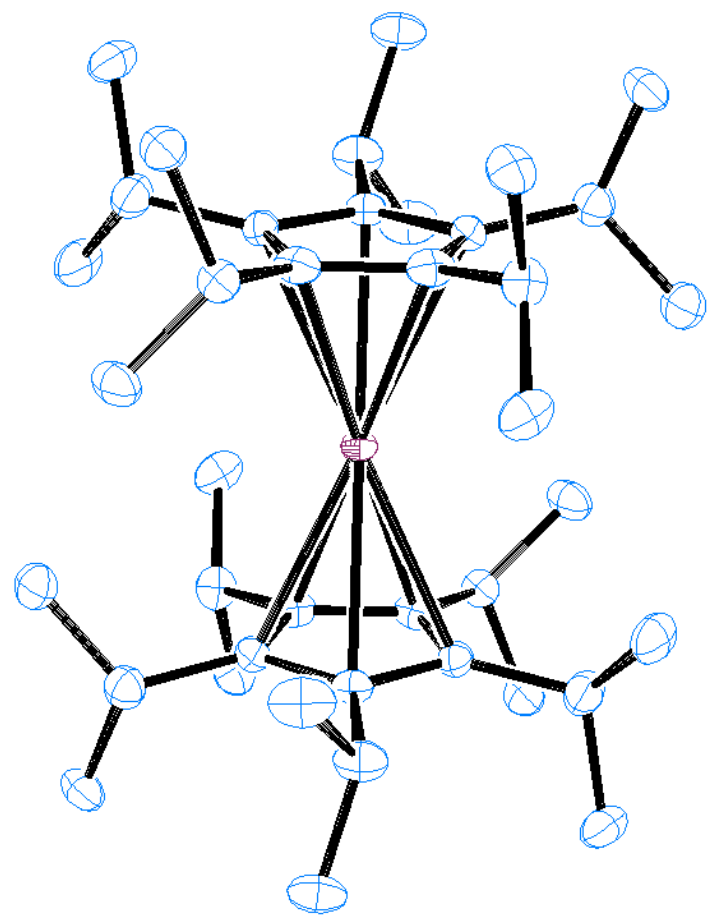

Figure S11. Solid-state structure of the $\mathrm{Tb}\left(\mathrm{Cp}^{i \operatorname{Pr} 5}\right)_{2}$ metallocene 2 with thermal ellipsoids at the $50 \%$ probability level. Hydrogen atoms are omitted for clarity. 


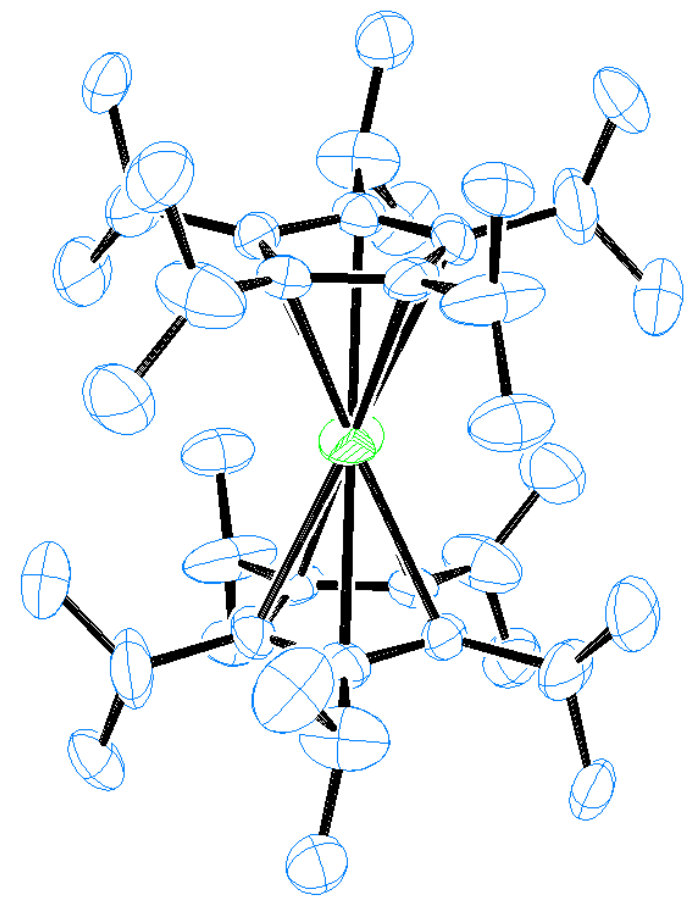

Figure S12. Solid-state structure of the $\operatorname{Dy}\left(\mathrm{Cp}^{i \mathrm{Pr} 5}\right)_{2}$ metallocene 3 with thermal ellipsoids at the $50 \%$ probability level. Hydrogen atoms are omitted for clarity. 
Table S2. Selected bond distances $(\AA)$ and angles $\left(^{\circ}\right)$ for $\mathbf{1}-\mathbf{3}$.

\begin{tabular}{|c|c|c|c|}
\hline & $\begin{array}{c}{\left[\mathrm{Tb}\left(\mathrm{Cp}^{\mathrm{iPr} 5}\right)_{2}\right]\left[\mathrm{B}\left(\mathrm{C}_{6} \mathrm{~F}_{5}\right)_{4}\right]} \\
(\mathbf{1})^{a}\end{array}$ & $\begin{array}{c}{\left[\mathrm{Tb}\left(\mathrm{Cp}^{\mathrm{iPr} 5}\right)_{2}\right]} \\
(\mathbf{2})\end{array}$ & $\begin{array}{c}{\left[\mathrm{Dy}\left(\mathrm{Cp}^{\mathrm{iPr} 5}\right)_{2}\right]} \\
(\mathbf{3})\end{array}$ \\
\hline $\mathrm{Ln}-\mathrm{C} 1$ & $2.660(7)$ & $2.703(2)$ & $2.662(5)$ \\
\hline $\mathrm{Ln}-\mathrm{C} 2$ & $2.625(7)$ & $2.704(2)$ & $2.682(5)$ \\
\hline $\mathrm{Ln}-\mathrm{C} 3$ & $2.616(8)$ & $2.711(2)$ & $2.680(4)$ \\
\hline $\mathrm{Ln}-\mathrm{C} 4$ & $2.600(7)$ & $2.703(2)$ & $2.680(4)$ \\
\hline $\mathrm{Ln}-\mathrm{C} 5$ & $2.662(8)$ & $2.702(2)$ & $2.660(4)$ \\
\hline $\mathrm{Ln}-\mathrm{C} 21$ & $2.587(8)$ & - & - \\
\hline $\mathrm{Ln}-\mathrm{C} 22$ & $2.702(7)$ & - & - \\
\hline $\mathrm{Ln}-\mathrm{C} 23$ & $2.725(7)$ & - & - \\
\hline $\mathrm{Ln}-\mathrm{C} 24$ & $2.634(9)$ & - & - \\
\hline $\mathrm{Ln}-\mathrm{C} 25$ & $2.536(9)$ & - & - \\
\hline $\mathrm{Ln}-\mathrm{C}(\operatorname{avg})^{b}$ & $2.635(8)$ & $2.705(2)$ & $2.673(4)$ \\
\hline $\mathrm{Ln}-\mathrm{Cp}(\mathrm{A})^{c}$ & $2.360(5)$ & $2.417(1)$ & $2.385(1)$ \\
\hline $\mathrm{Ln}-\mathrm{Cp}(\mathrm{B})^{c}$ & $2.353(6)$ & - & - \\
\hline $\mathrm{Ln}-\mathrm{Cp}(\mathrm{avg})^{b, c}$ & $2.356(6)$ & $2.417(1)$ & $2.385(1)$ \\
\hline $\mathrm{Cp}(\mathrm{A})-\mathrm{Ln}-\mathrm{Cp}(\mathrm{B})^{c}$ & $159.8(4)$ & 180.0 & 180.0 \\
\hline
\end{tabular}

${ }^{b}$ Values for $\mathbf{1}$ are averages over the four positions of the metal atom.

${ }^{b}$ The standard deviation of the average value was estimated from $\sigma=\sqrt{\sum \sigma_{i}^{2} / N}$, where $\sigma_{\mathrm{i}}$ is the standard deviation of each bond distance $i$ and $N$ is the number of distances averaged.

${ }^{c} \mathrm{Ln}-\mathrm{Cp}$ distances and angles were measured from the $\mathrm{Ln}$ atom to the centroid of the $\mathrm{Cp} \operatorname{ring}(\mathrm{s})$. 


\section{Magnetic Measurements}

General. Magnetic samples were prepared by adding crystalline powder $(18.2 \mathrm{mg}$ of $\mathbf{1}, 18.2 \mathrm{mg}$ of $2,22.0 \mathrm{mg}$ of 3 ) to a $5 \mathrm{~mm}$ i.d. $/ 7 \mathrm{~mm}$ o.d. quartz tube with a raised quartz platform. A layer of eicosane was added to the samples $(20.4 \mathrm{mg}$ for $\mathbf{1}, 29.9 \mathrm{mg}$ for $\mathbf{2}, 30.5 \mathrm{mg}$ for 3 ) to provide good thermal contact between the sample and the bath and to prevent crystallite torqueing. The tubes were fitted with Teflon sealable adapters, evacuated using a glovebox vacuum pump, and then flame sealed with an $\mathrm{O}_{2} / \mathrm{H}_{2}$ flame under vacuum. After flame-sealing, the eicosane was melted in a $40{ }^{\circ} \mathrm{C}$ water bath.

Magnetic susceptibility measurements were collected using a Quantum Design MPMS2 SQUID magnetometer. All data were corrected for diamagnetic contributions from the core diamagnetism and for the diamagnetism of the eicosane used to suspend the sample, estimated using Pascal's constants to give corrections of $\chi_{\mathrm{dia}}=-0.000741 \mathrm{emu} / \mathrm{mol},-0.000464 \mathrm{emu} / \mathrm{mol}$, and -0.000464 $\mathrm{emu} / \mathrm{mol}$ for $\mathbf{1}, \mathbf{2}$, and $\mathbf{3}$, respectively.

Magnetic measurements were also conducted on frozen toluene solutions of $\mathbf{2}$ and $\mathbf{3}$ (19 mM and $28 \mathrm{mM}$, respectively) to ensure that the observed magnetic properties were molecular in origin and not due to long-range interactions in the solid-state. These frozen solutions correspond to an average $\mathrm{Tb} \cdots \mathrm{Tb}$ separation of $44 \AA$ in $19 \mathrm{mM} 2$ and an average Dy $\cdots$ Dy separation of $39 \AA$ in 28 $\mathrm{mM} 3$.

Dc Magnetic Susceptibility Measurements. Dc magnetic susceptibility measurements were performed at temperatures ranging from 2 to $300 \mathrm{~K}$ under applied fields of 1,5 , and $10 \mathrm{kOe}(0.1$, 0.5 , and $1 \mathrm{~T}$ ) for 1-3.

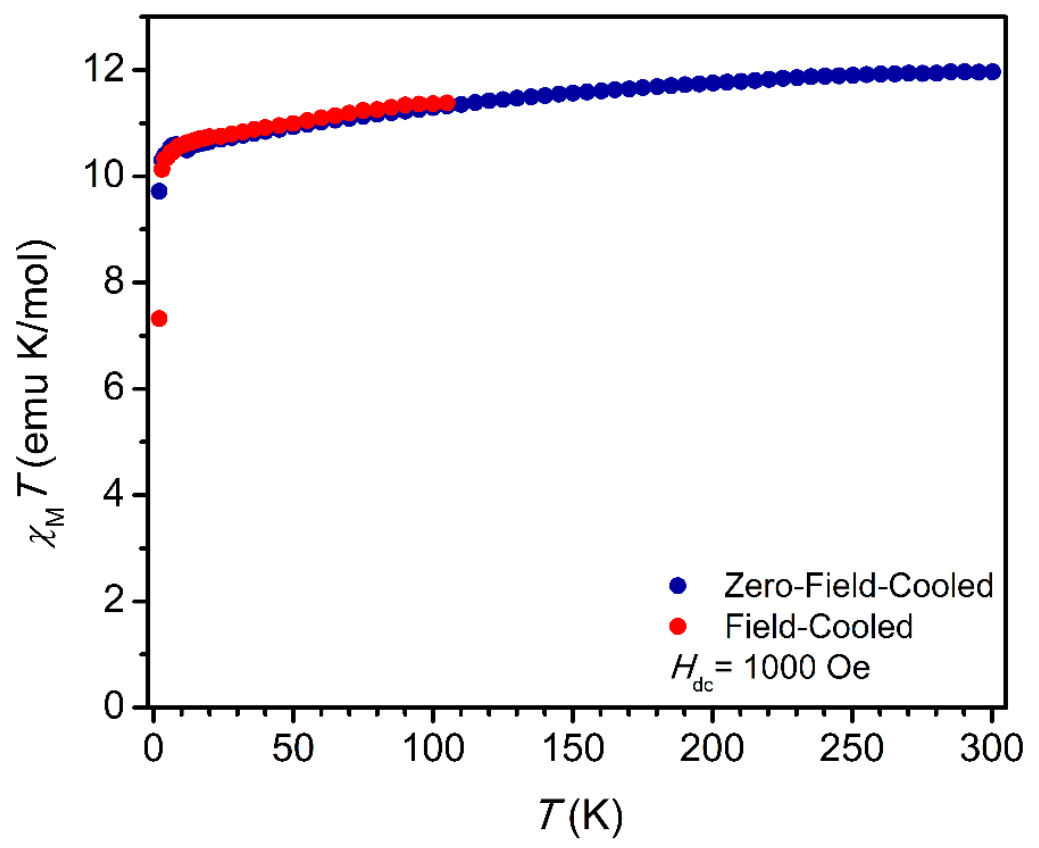

Figure S13. Field-cooled (red) and zero-field cooled (blue) measurements of $\mathbf{1}$ under an applied field of $1 \mathrm{kOe}$. 


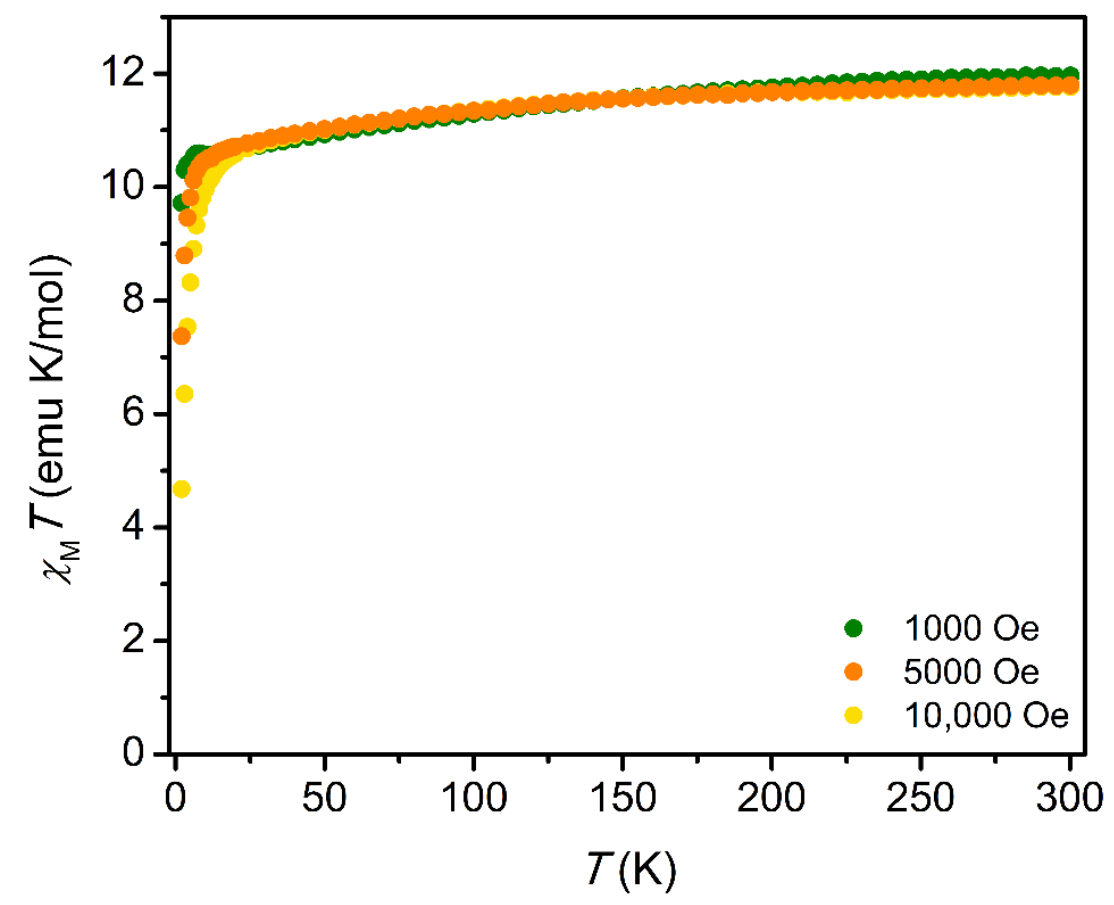

Figure S14. Zero-field cooled dc magnetic susceptibility measurements of 1 under applied fields of 1 (green), 5 (orange), and $10 \mathrm{kOe}$ (yellow).

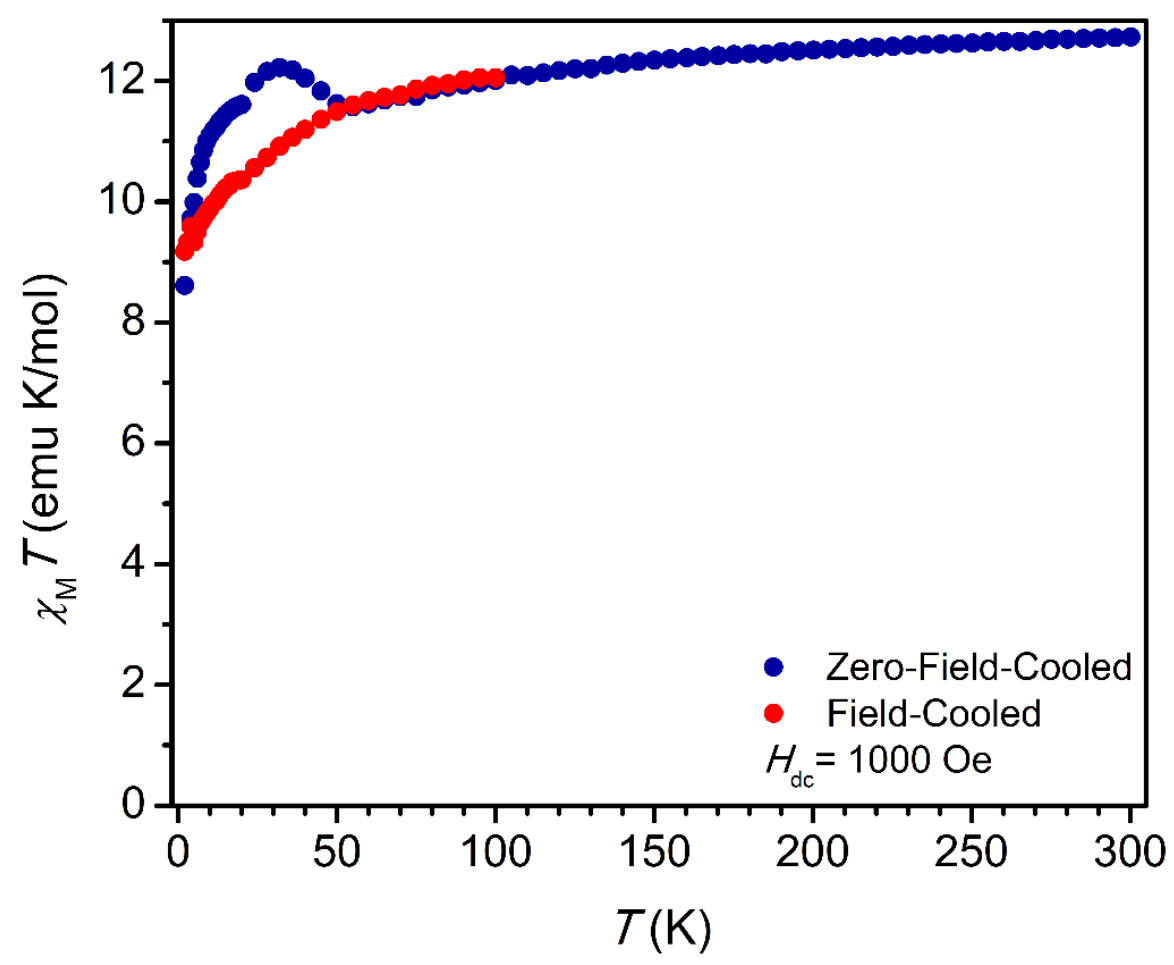

Figure S15. Field-cooled (red) and zero-field cooled (blue) measurements of $\mathbf{2}$ under an applied field of $1 \mathrm{kOe}$. 


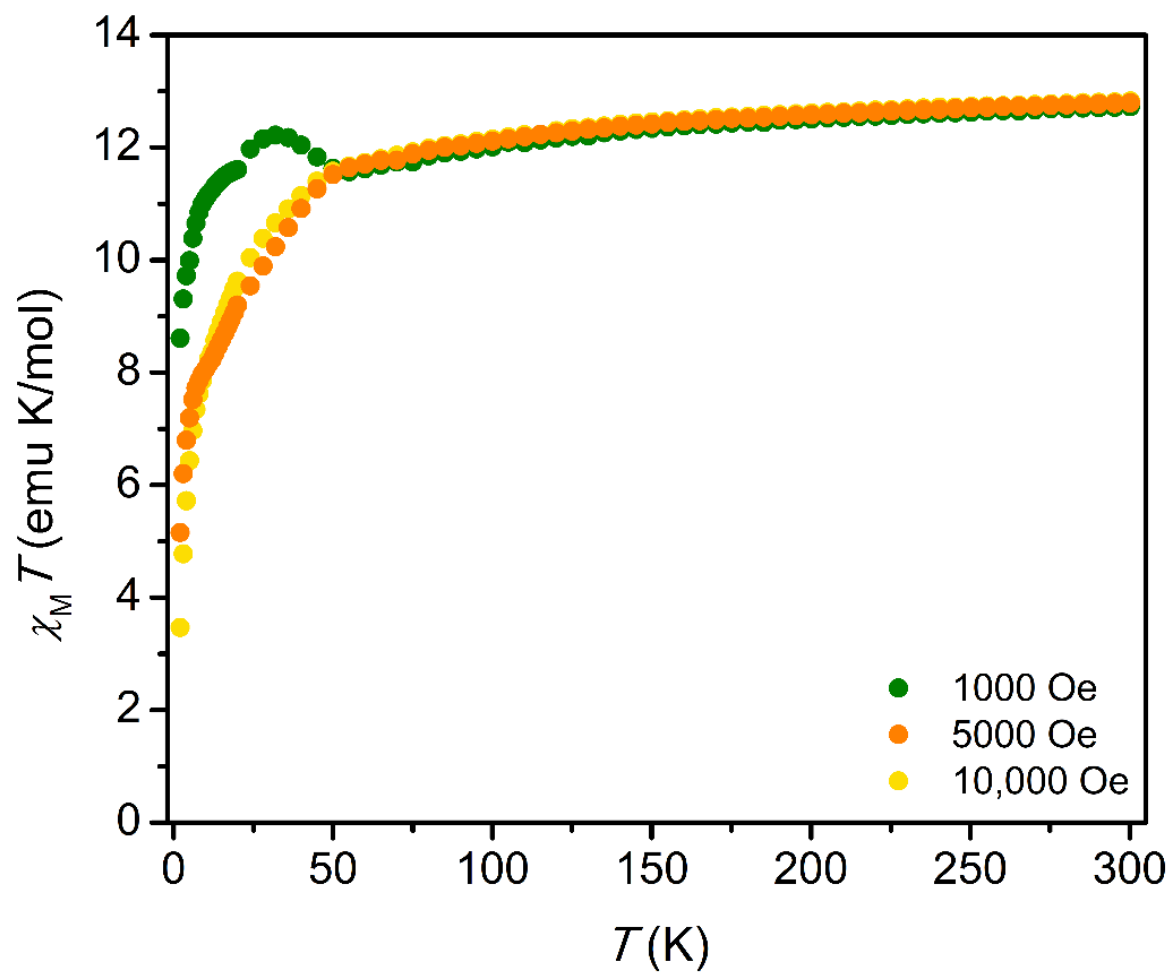

Figure S16. Zero-field cooled dc magnetic susceptibility measurements of $\mathbf{2}$ under applied fields of 1 (green), 5 (orange), and $10 \mathrm{kOe}$ (yellow).

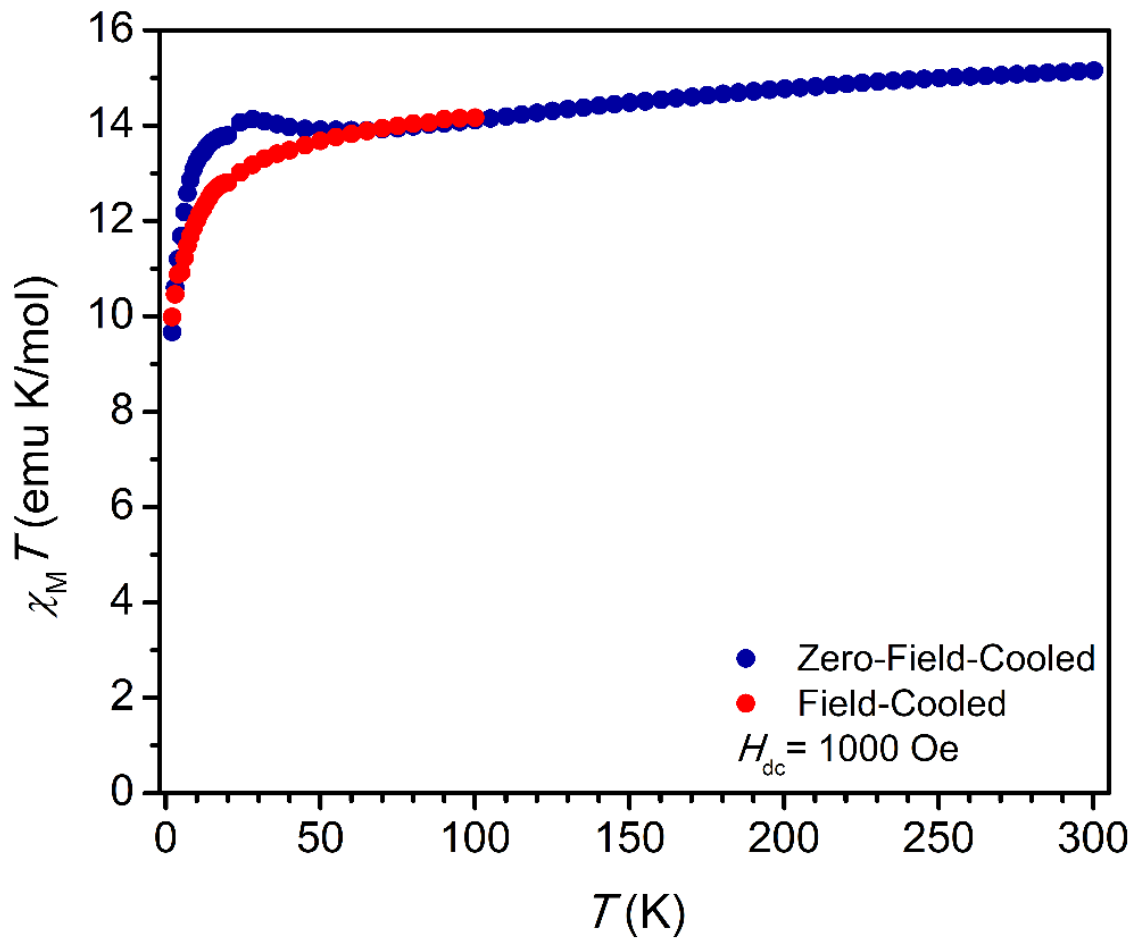

Figure S17. Field-cooled (red) and zero-field cooled (blue) measurements of $\mathbf{3}$ under an applied field of $1 \mathrm{kOe}$. 


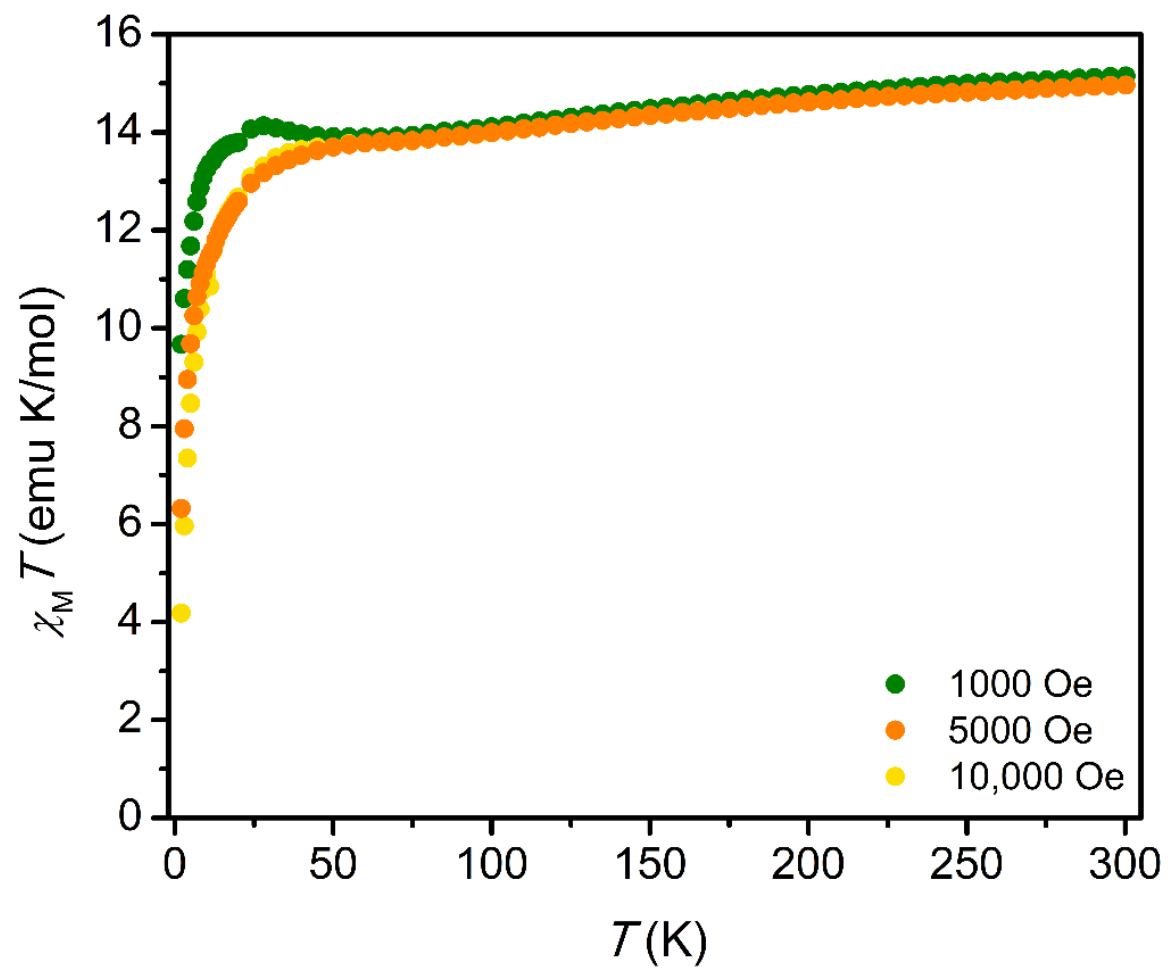

Figure S18. Zero-field cooled dc magnetic susceptibility measurements of $\mathbf{3}$ under applied fields of 1 (green), 5 (orange), and $10 \mathrm{kOe}$ (yellow). 
Ac Magnetic Susceptibility Measurements. Ac magnetic susceptibility measurements were performed with a probe field of 4 Oe at frequencies ranging from 0.1 to $1500 \mathrm{~Hz}$ under external applied fields of 0 and 500 Oe $(0,0.05 \mathrm{~T})$ for 1-3. Magnetic relaxation times, $\tau$, were extracted from a simultaneous fit of in-phase $\left(\chi_{\mathrm{m}^{\prime}}\right)$ and out-of-phase $\left(\chi_{\mathrm{m}^{\prime \prime}}\right)$ components of the magnetic susceptibility to a generalized Debye model.
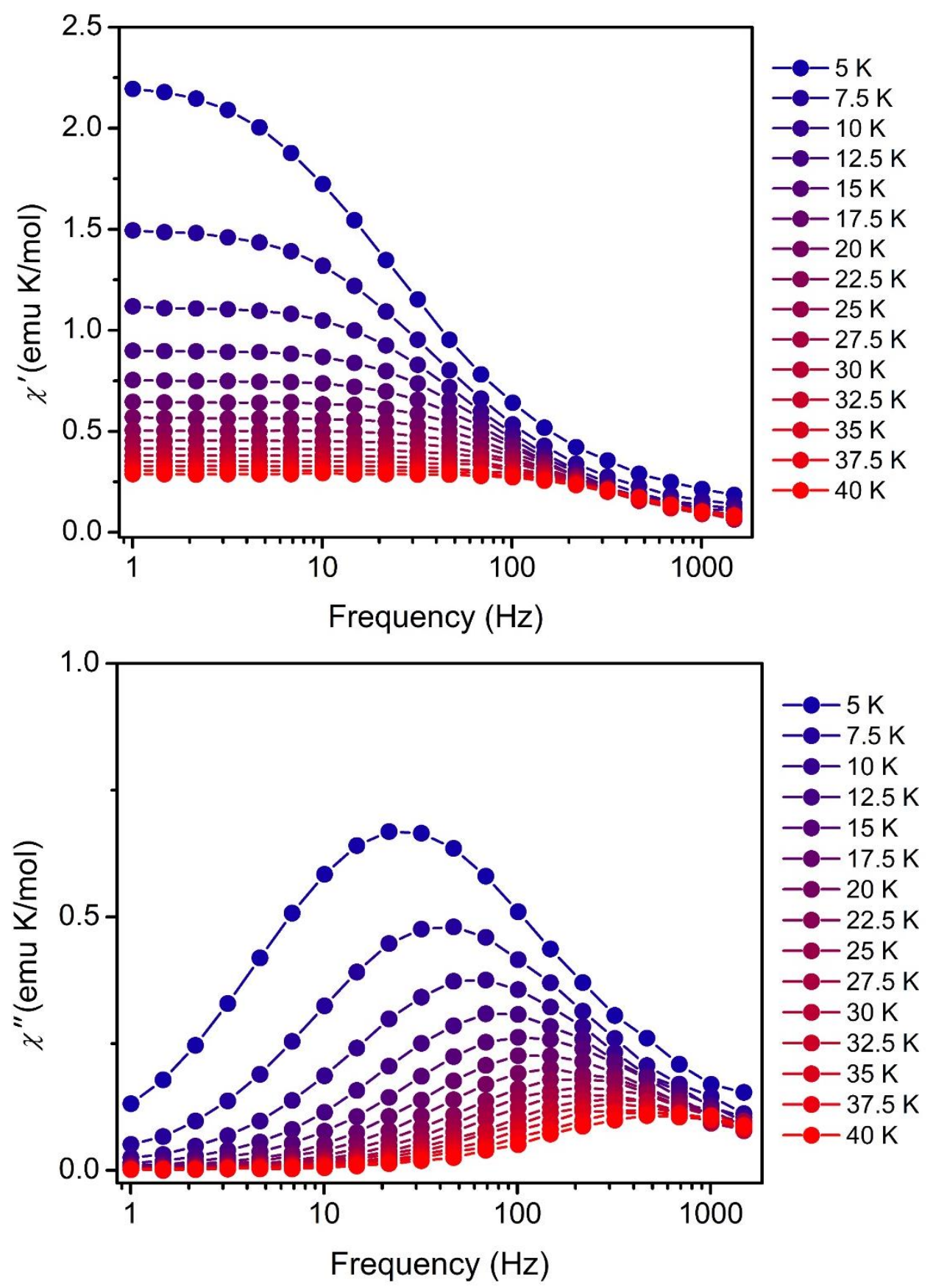

Figure S19. In-phase $\left(\chi_{\mathrm{m}^{\prime}}\right.$, top) and out-of-phase $\left(\chi_{\mathrm{M}}{ }^{\prime \prime}\right.$, bottom) components of the ac magnetic susceptibility for 1 under zero applied dc field at frequencies ranging from 1-1500 $\mathrm{Hz}$ and temperatures from 5-40 K ( $2.5 \mathrm{~K}$ steps). The colored lines are guides for the eye. 


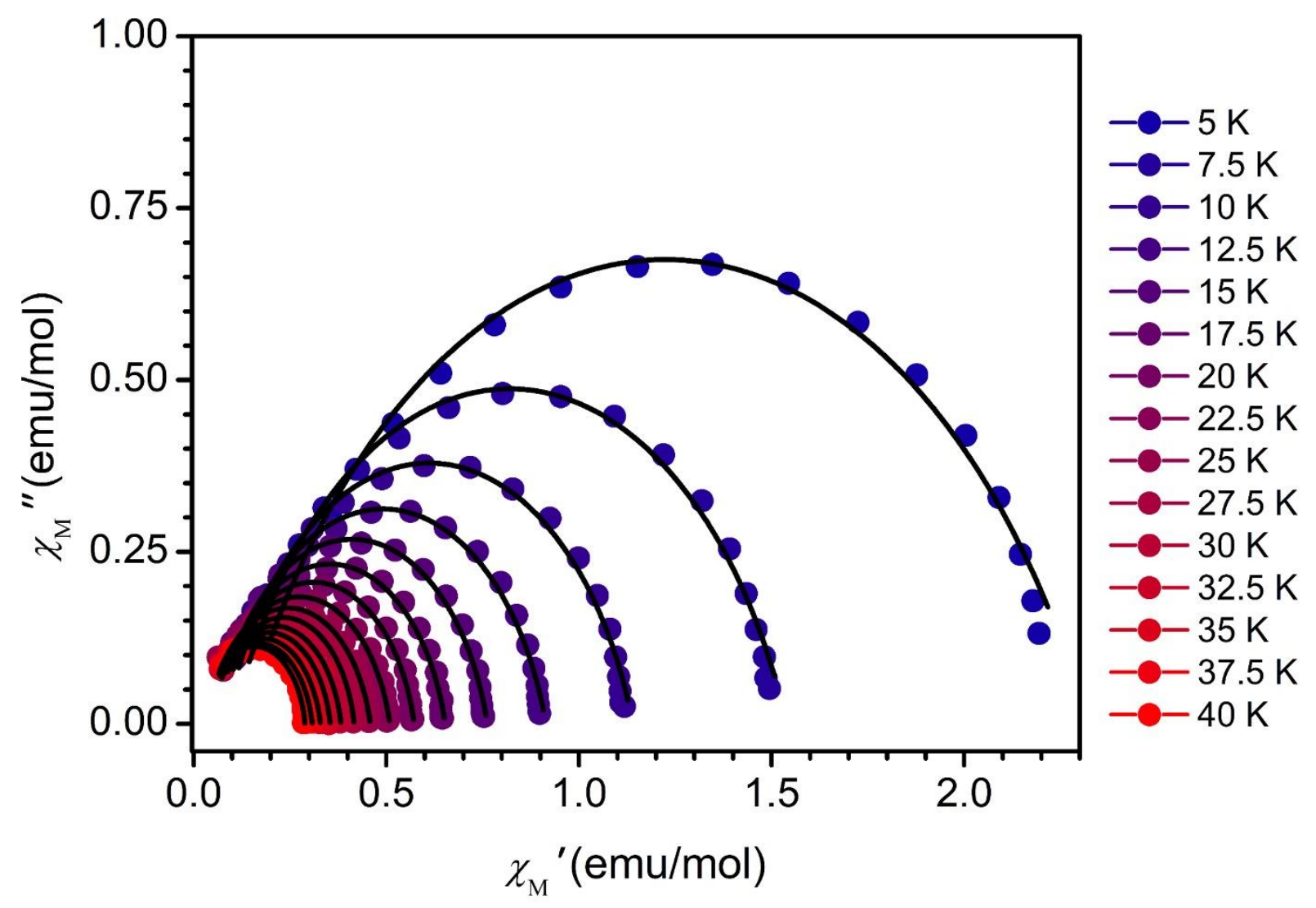

Figure S20. Cole-Cole plots for 1 from $5-40 \mathrm{~K}$ under zero applied dc field ( $2.5 \mathrm{~K}$ steps). The black lines represent fits to the data using a generalized Debye model, which were used to extract $\tau$ values at each temperature. 

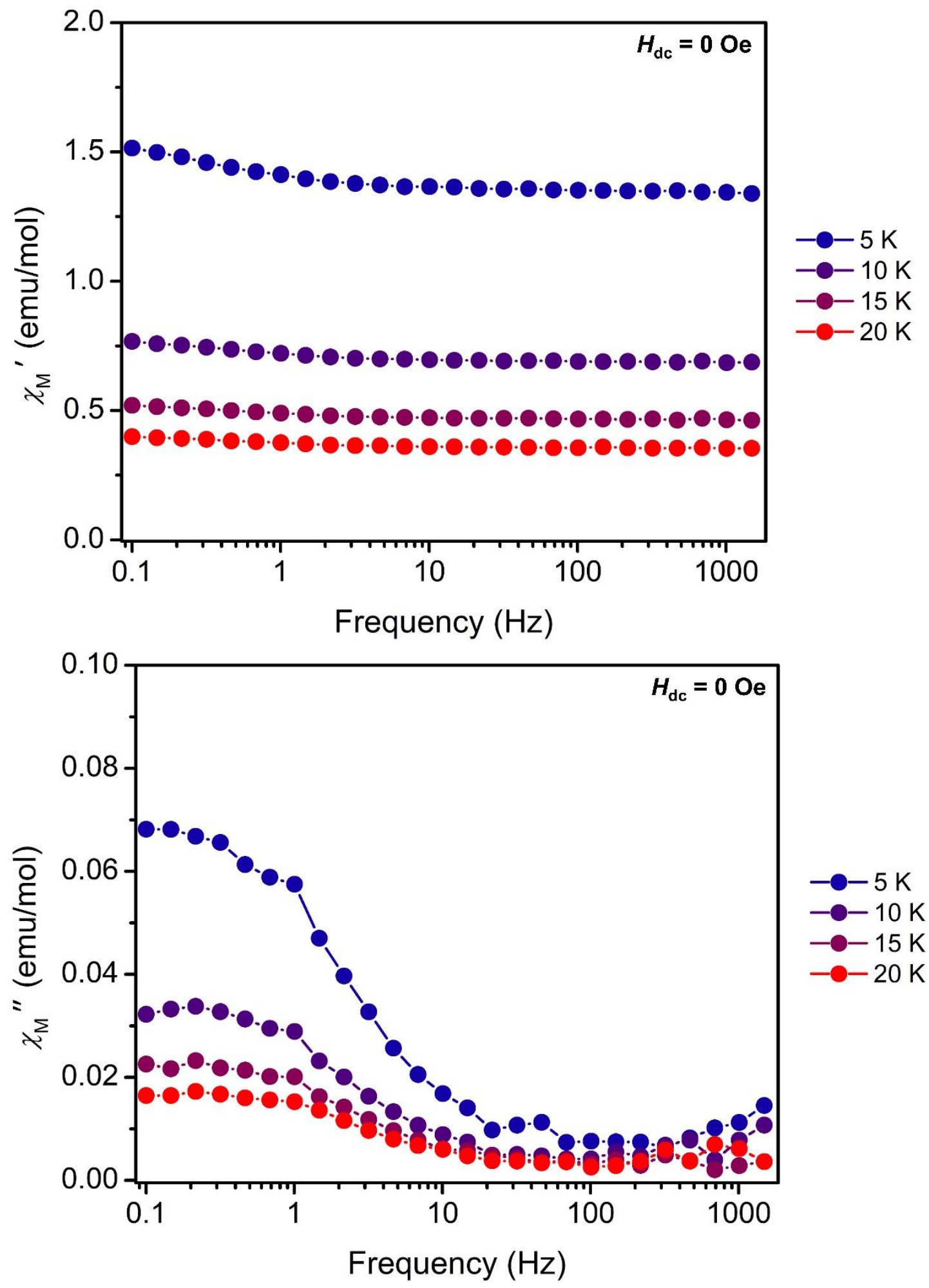

Figure S21. In-phase $\left(\chi_{\mathrm{M}^{\prime}}\right.$, top) and out-of-phase $\left(\chi_{\mathrm{M}}{ }^{\prime \prime}\right.$, bottom) components of the ac magnetic susceptibility for 2 under zero applied dc field at frequencies ranging from $0.1-1500 \mathrm{~Hz}$ and temperatures from $5-20 \mathrm{~K}$ ( $5 \mathrm{~K}$ steps). The colored lines are guides for the eye. 


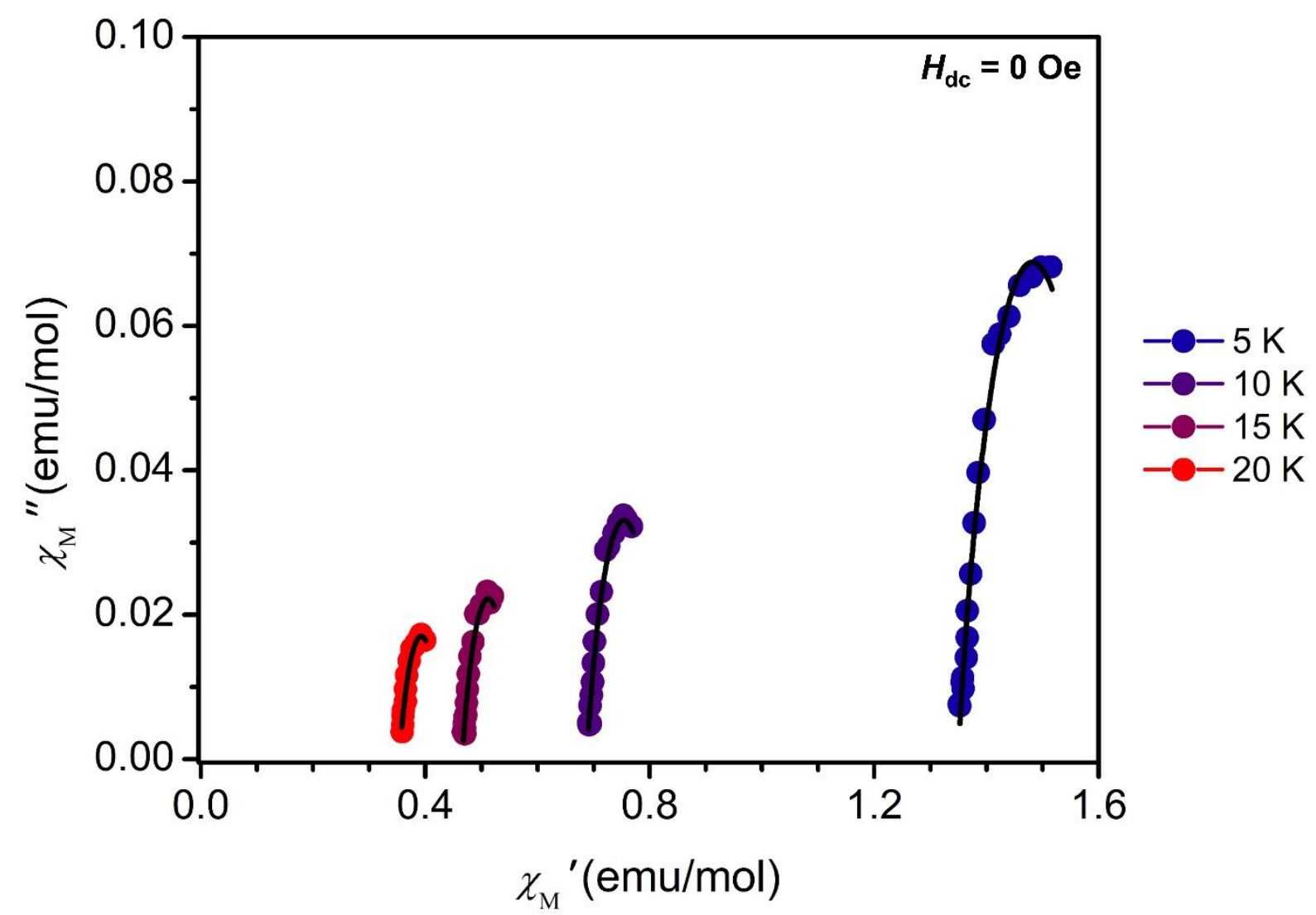

Figure S22. Cole-Cole plots for 2 from 5-20 K under zero applied dc field ( $5 \mathrm{~K}$ steps). The black lines represent fits to the data using a generalized Debye model, which were used to extract $\tau$ values at each temperature. 

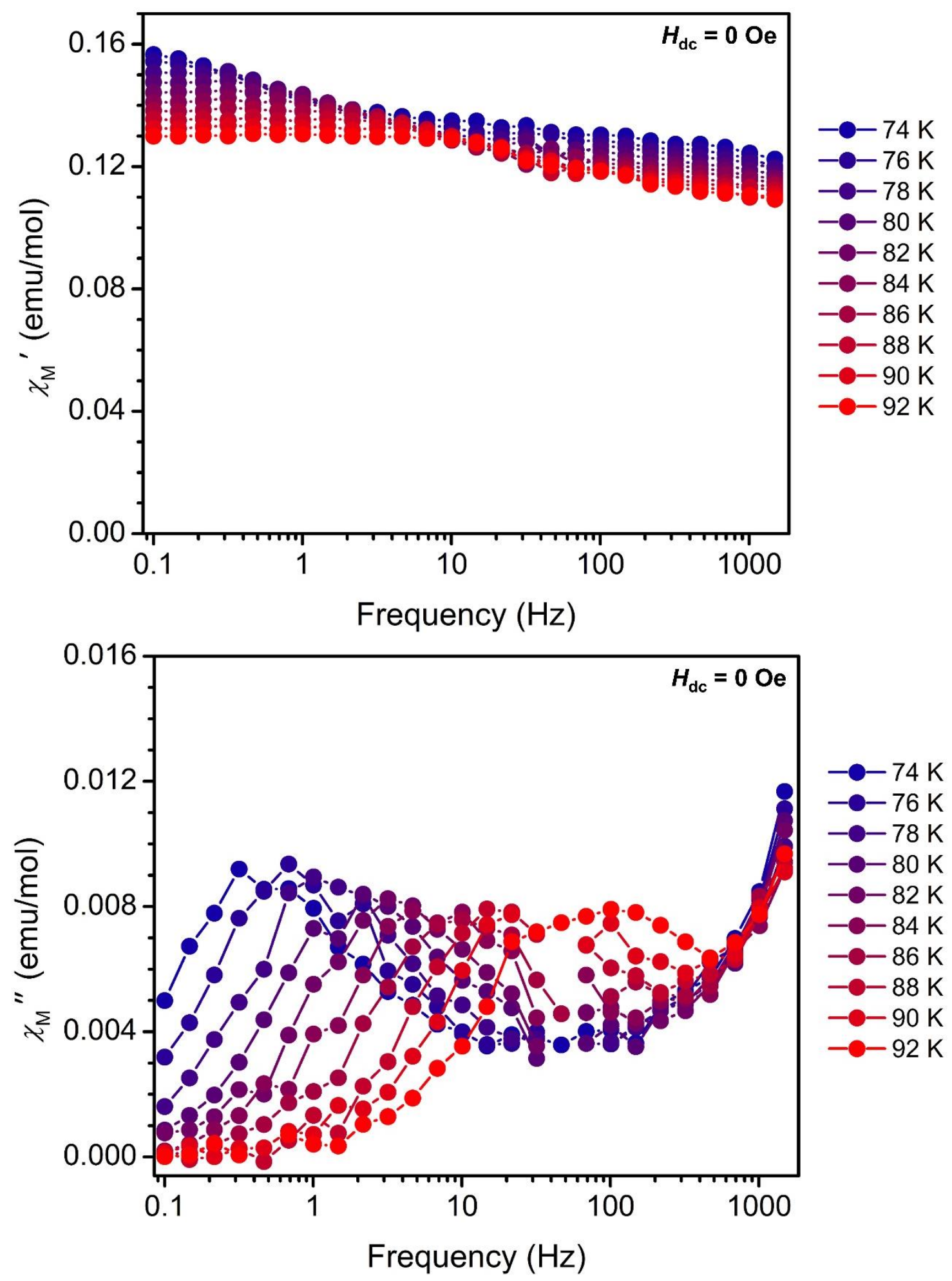

Figure S23. In-phase $\left(\chi_{\mathrm{M}^{\prime}}\right.$, top) and out-of-phase $\left(\chi_{\mathrm{M}}{ }^{\prime \prime}\right.$, bottom) components of the ac magnetic susceptibility for 2 under zero applied dc field at frequencies ranging from $0.1-1500 \mathrm{~Hz}$ and temperatures from 74-92 K ( $2 \mathrm{~K}$ steps). The colored lines are guides for the eye. 


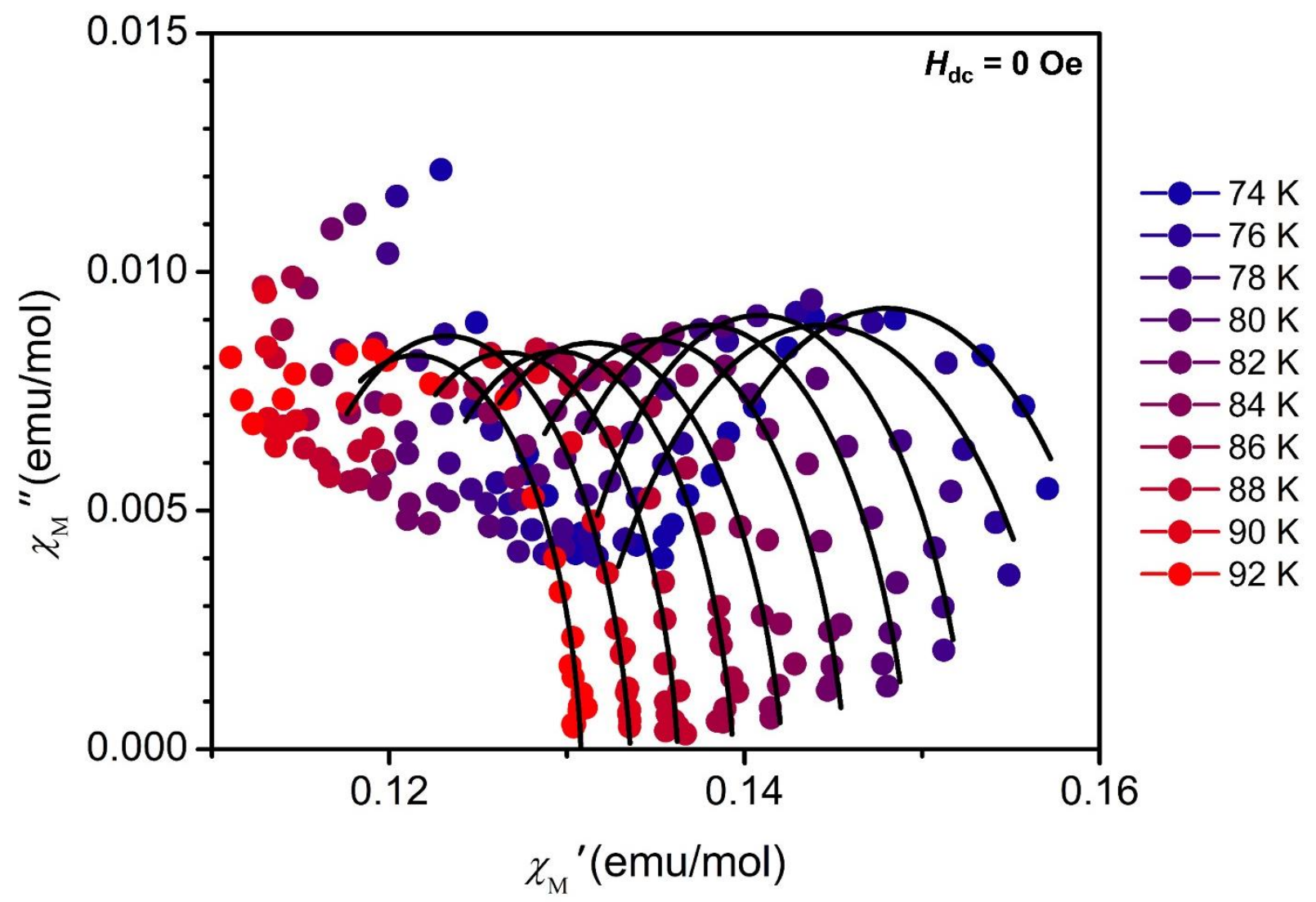

Figure S24. Cole-Cole plots for 2 from 74-92 K under zero applied dc field ( $2 \mathrm{~K}$ steps). The black lines represent fits to the data using a generalized Debye model, which were used to extract $\tau$ values at each temperature. 

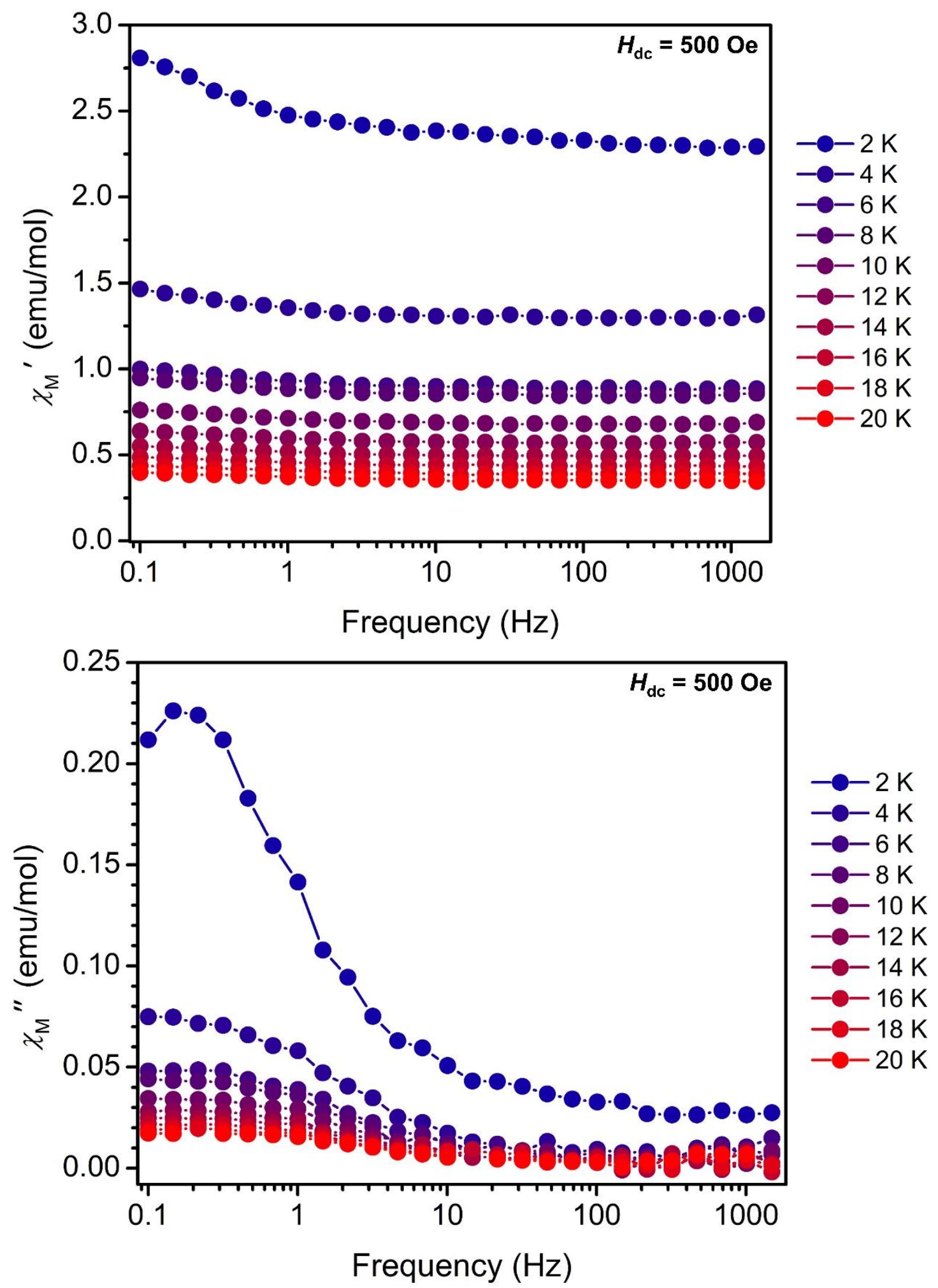

Figure S25. In-phase $\left(\chi_{\mathrm{M}^{\prime}}\right.$, top) and out-of-phase $\left(\chi_{\mathrm{M}}{ }^{\prime \prime}\right.$, bottom) components of the ac magnetic susceptibility for 2 under a 500 Oe applied dc field at frequencies ranging from $0.1-1500 \mathrm{~Hz}$ and temperatures from $2-20 \mathrm{~K}$ ( $2 \mathrm{~K}$ steps). The colored lines are guides for the eye. 


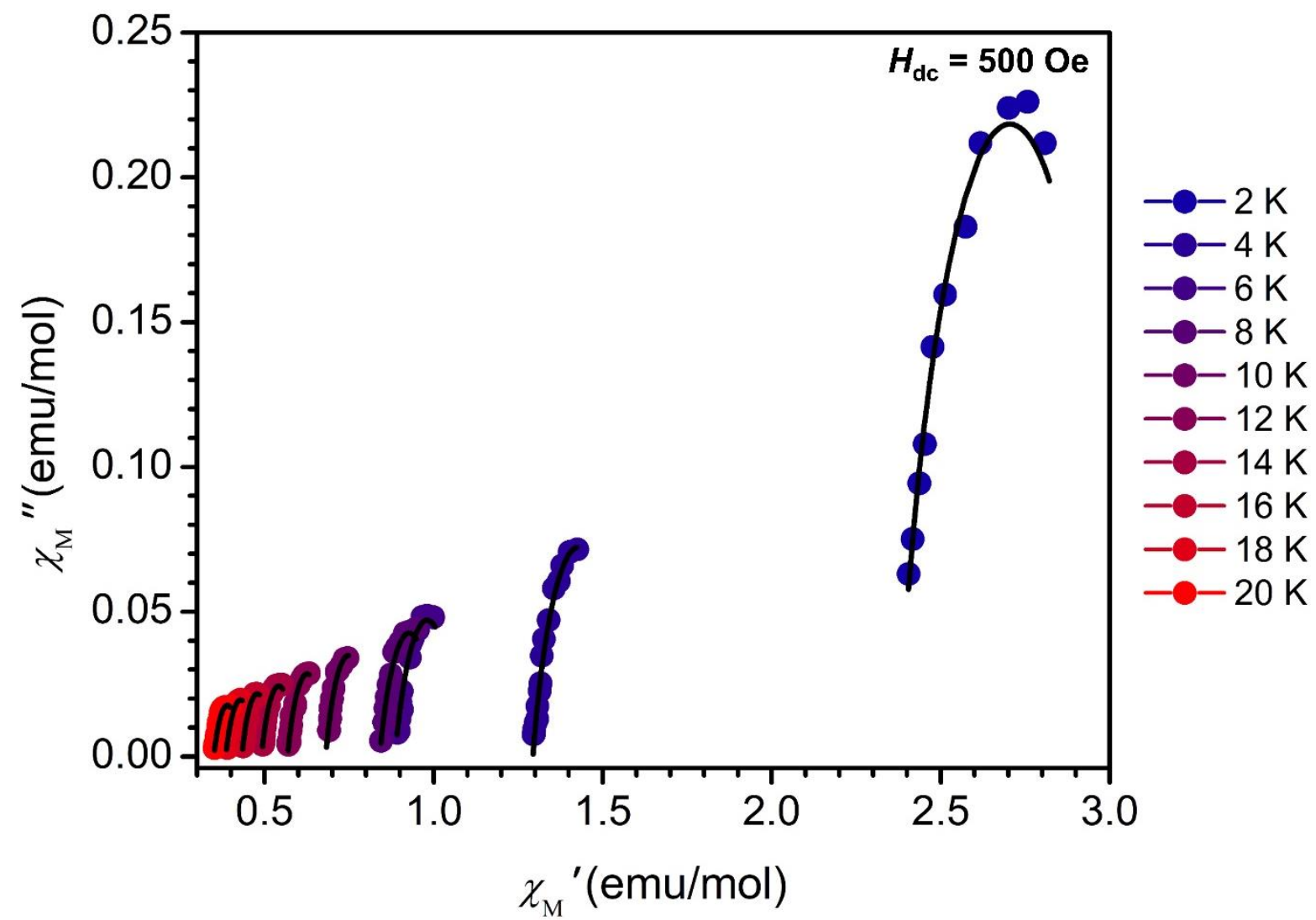

Figure S26. Cole-Cole plots for 2 from 2-20 K under a 500 Oe applied de field (2 K steps). The black lines represent fits to the data using a generalized Debye model, which were used to extract $\tau$ values at each temperature. 

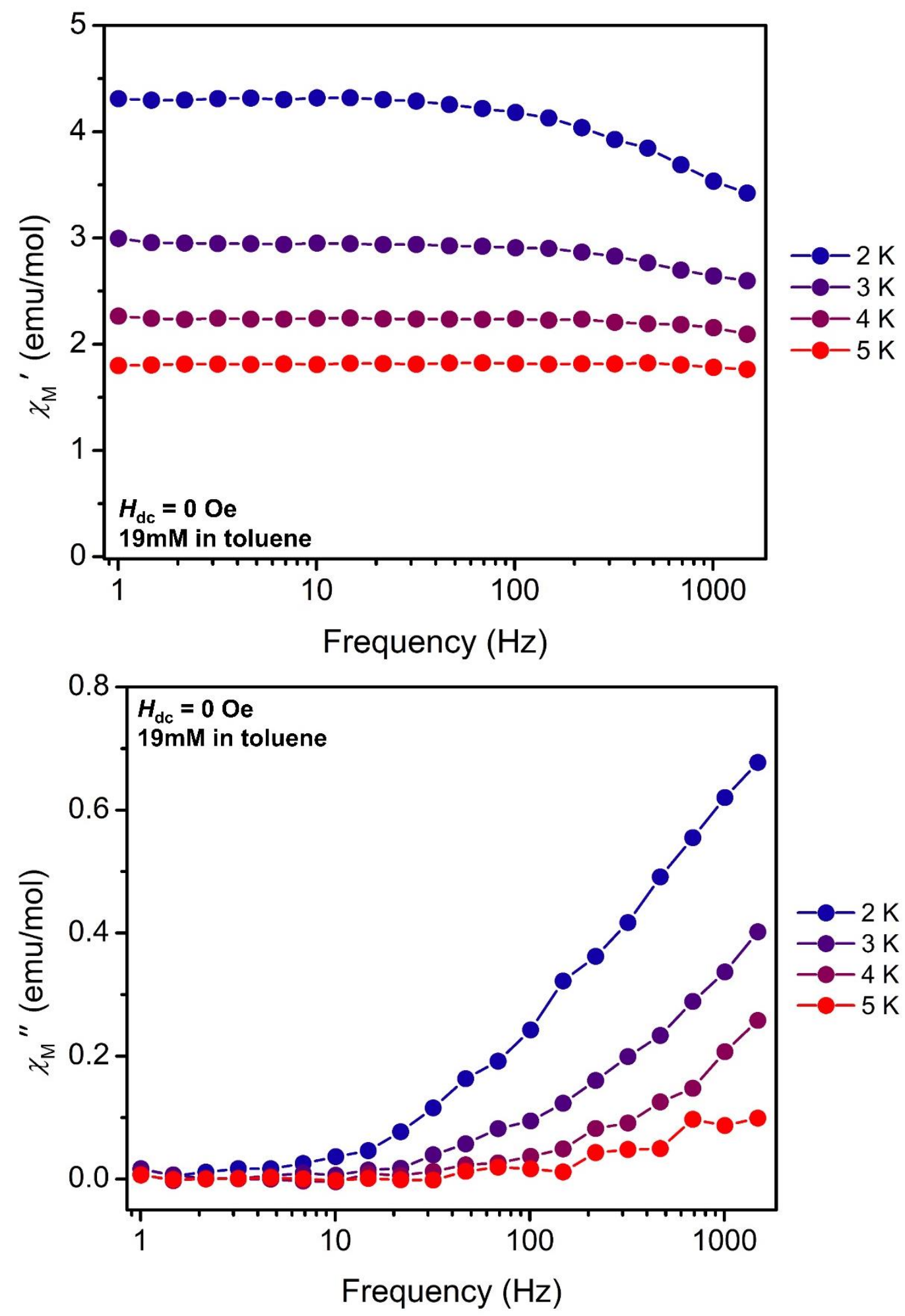

Figure S27. In-phase $\left(\chi_{\mathrm{M}}{ }^{\prime}\right.$, top) and out-of-phase ( $\chi_{\mathrm{M}}{ }^{\prime \prime}$, bottom) components of the ac magnetic susceptibility for a $19 \mathrm{mM}$ solution of 2 in toluene under zero applied dc field at frequencies ranging from $1-1500 \mathrm{~Hz}$ and temperatures from $2-5 \mathrm{~K}$ (1 K steps). The colored lines are guides for the eye. 


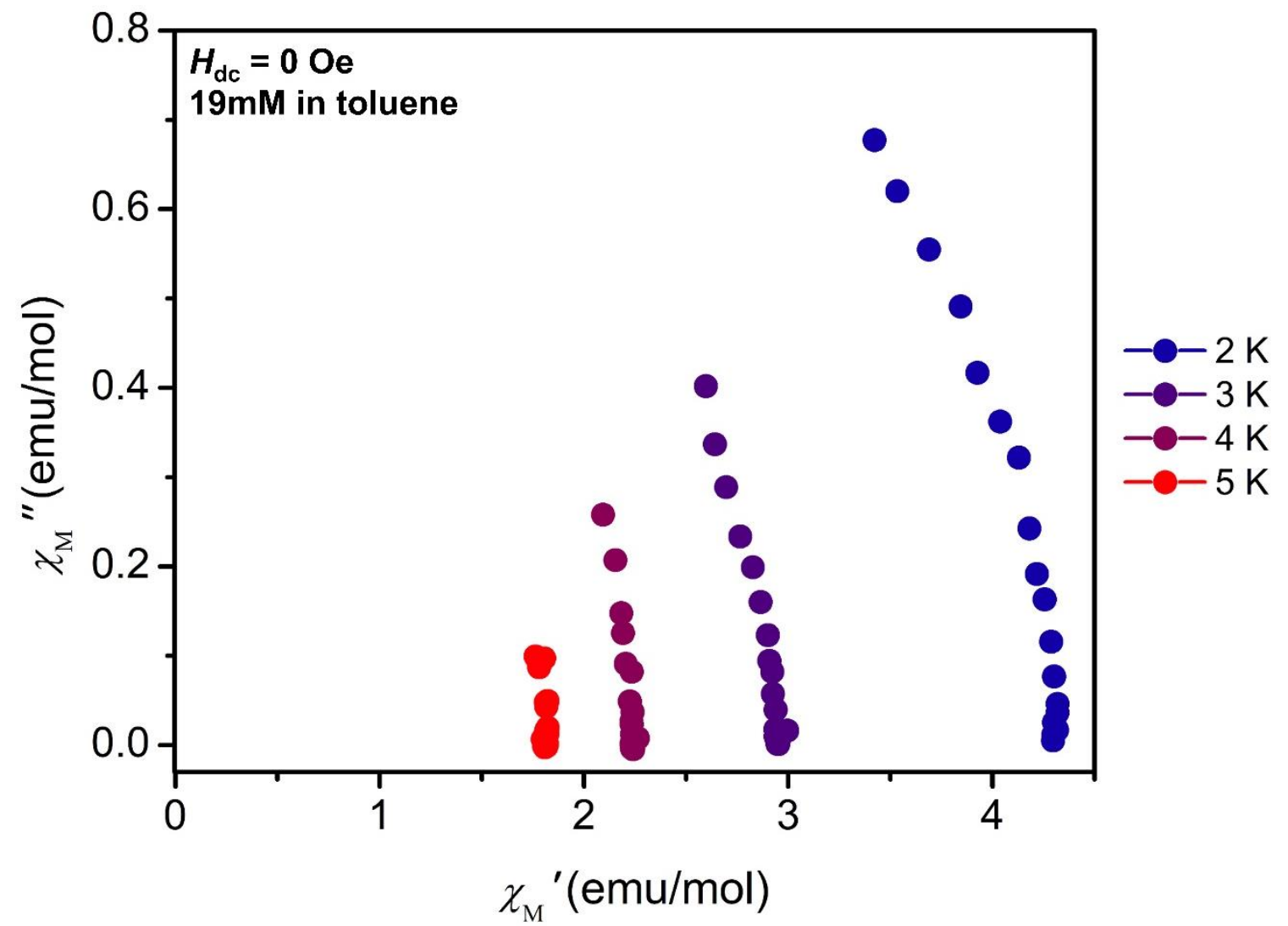

Figure S28. Cole-Cole plots for a $19 \mathrm{mM}$ solution of 2 in toluene from $2-5 \mathrm{~K}$ under zero applied dc field (1 K steps). 

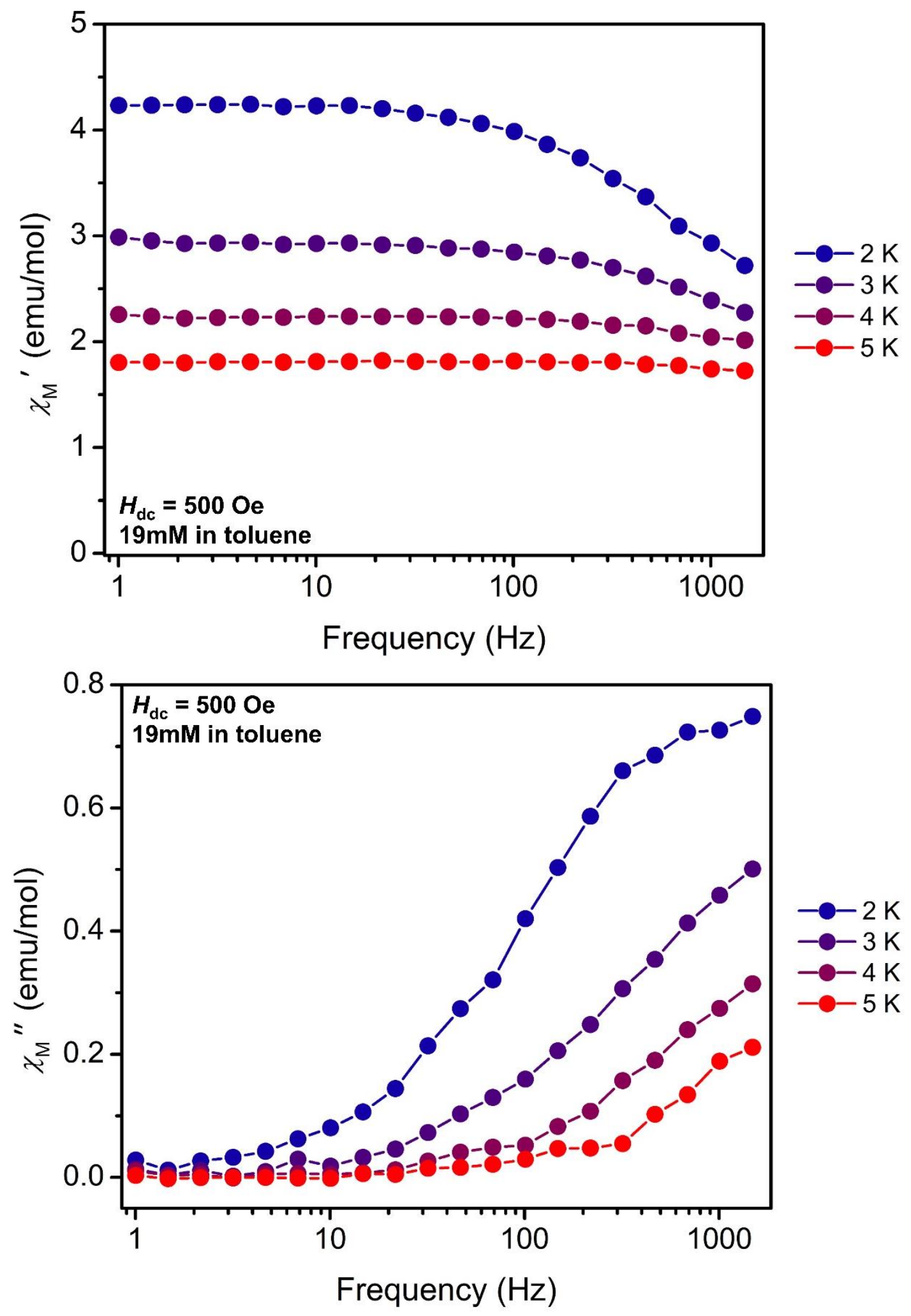

Figure S29. In-phase $\left(\chi_{\mathrm{M}^{\prime}}\right.$, top) and out-of-phase ( $\chi_{\mathrm{M}}{ }^{\prime \prime}$, bottom) components of the ac magnetic susceptibility for a $19 \mathrm{mM}$ solution of 2 in toluene under a 500 Oe applied dc field at frequencies ranging from $1-1500 \mathrm{~Hz}$ and temperatures from $2-5 \mathrm{~K}$ (1 K steps). The colored lines are guides for the eye. 


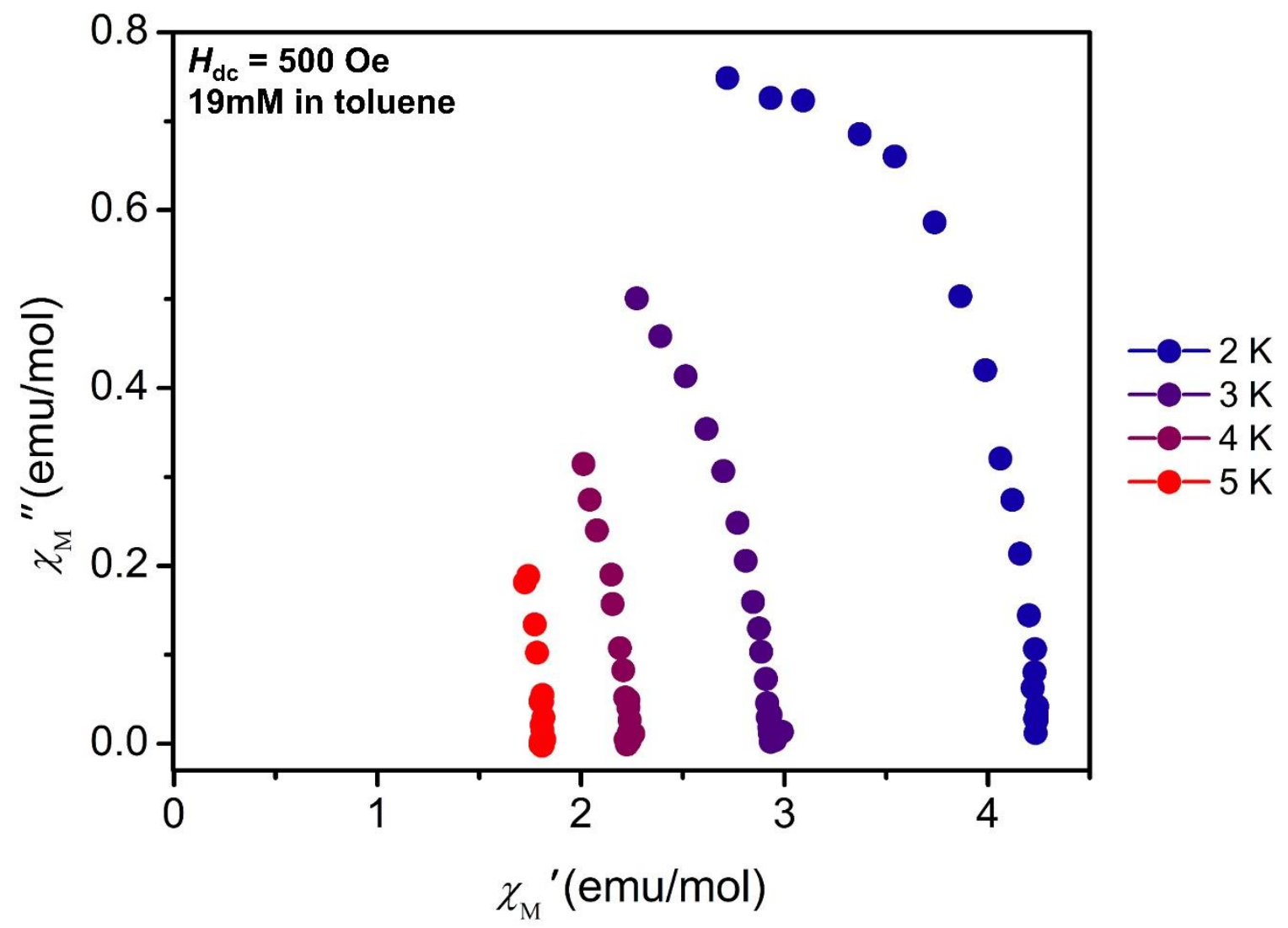

Figure S30. Cole-Cole plots for a $19 \mathrm{mM}$ solution of $\mathbf{2}$ in toluene from 2-5 K under a 500 Oe applied dc field (1 K steps). 

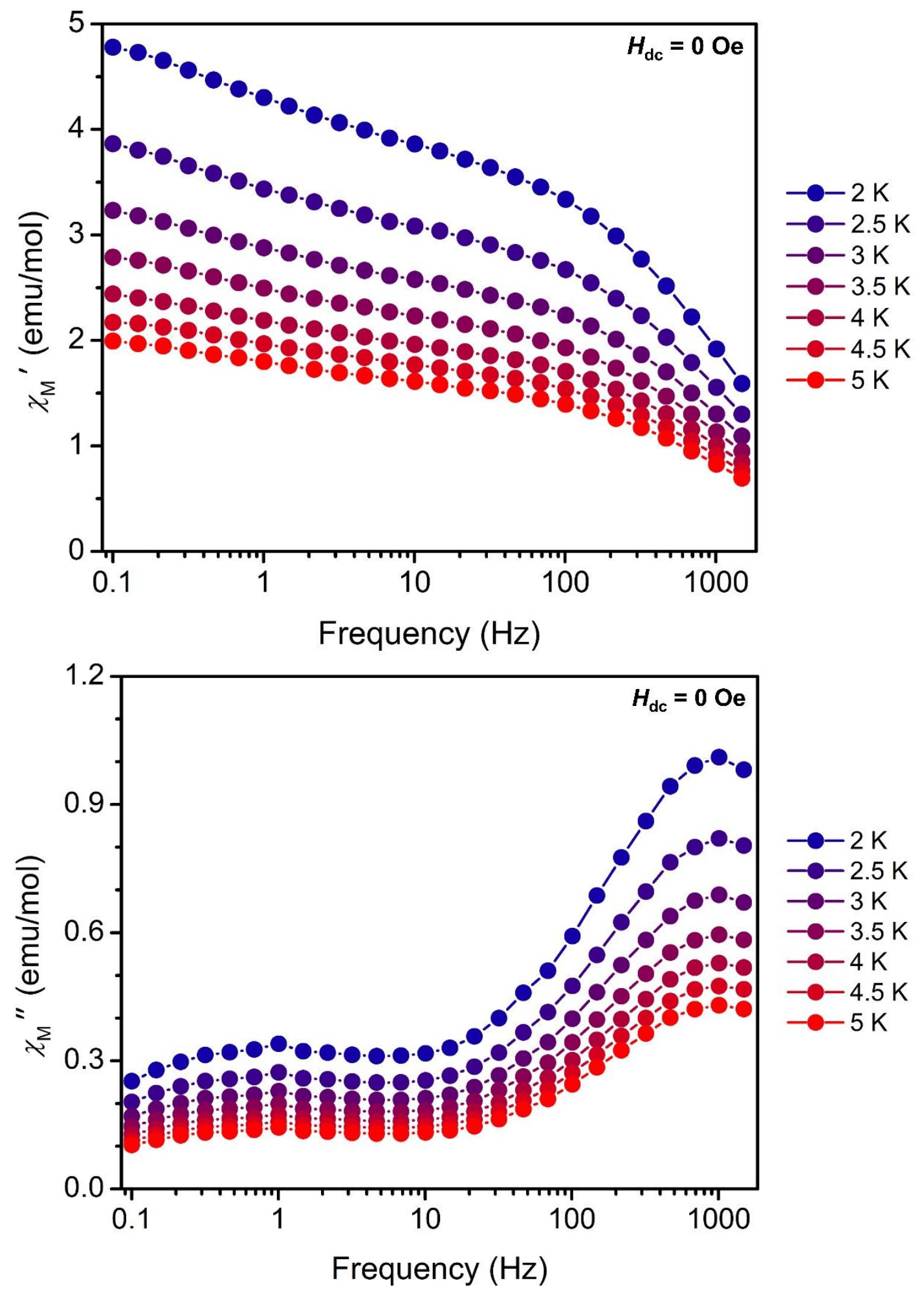

Figure S31. In-phase $\left(\chi_{\mathrm{M}^{\prime}}\right.$, top) and out-of-phase $\left(\chi_{\mathrm{M}}{ }^{\prime \prime}\right.$, bottom) components of the ac magnetic susceptibility for 3 under zero applied dc field at frequencies ranging from $0.1-1500 \mathrm{~Hz}$ and temperatures from $2-5 \mathrm{~K}(0.5 \mathrm{~K}$ steps). The colored lines are guides for the eye. 


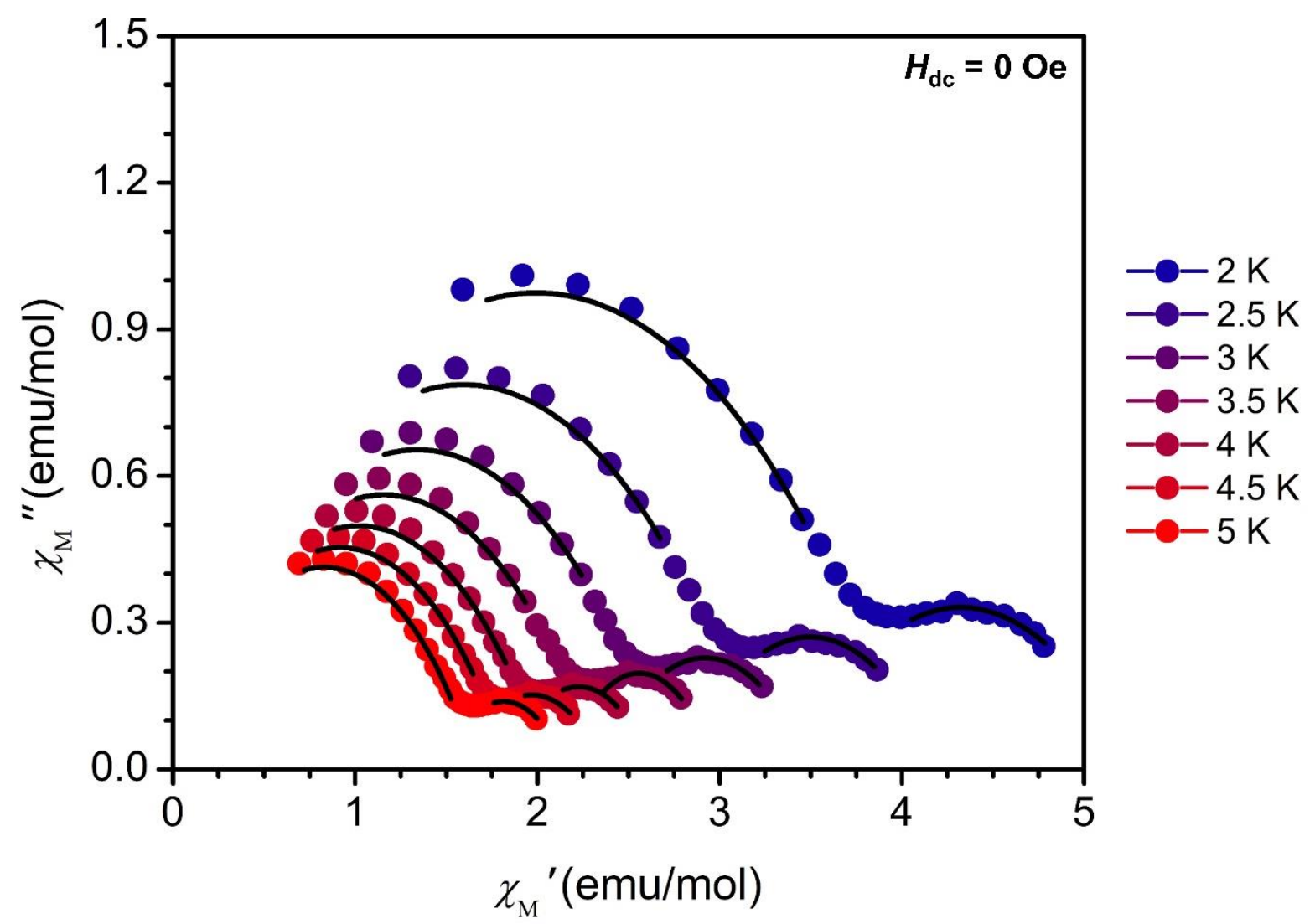

Figure S32. Cole-Cole plots for 3 from $2-5 \mathrm{~K}$ under zero applied dc field ( $0.5 \mathrm{~K}$ steps). The black lines represent fits to the data using a generalized Debye model, which were used to extract $\tau$ values at each temperature. Data was fit using two processes. 

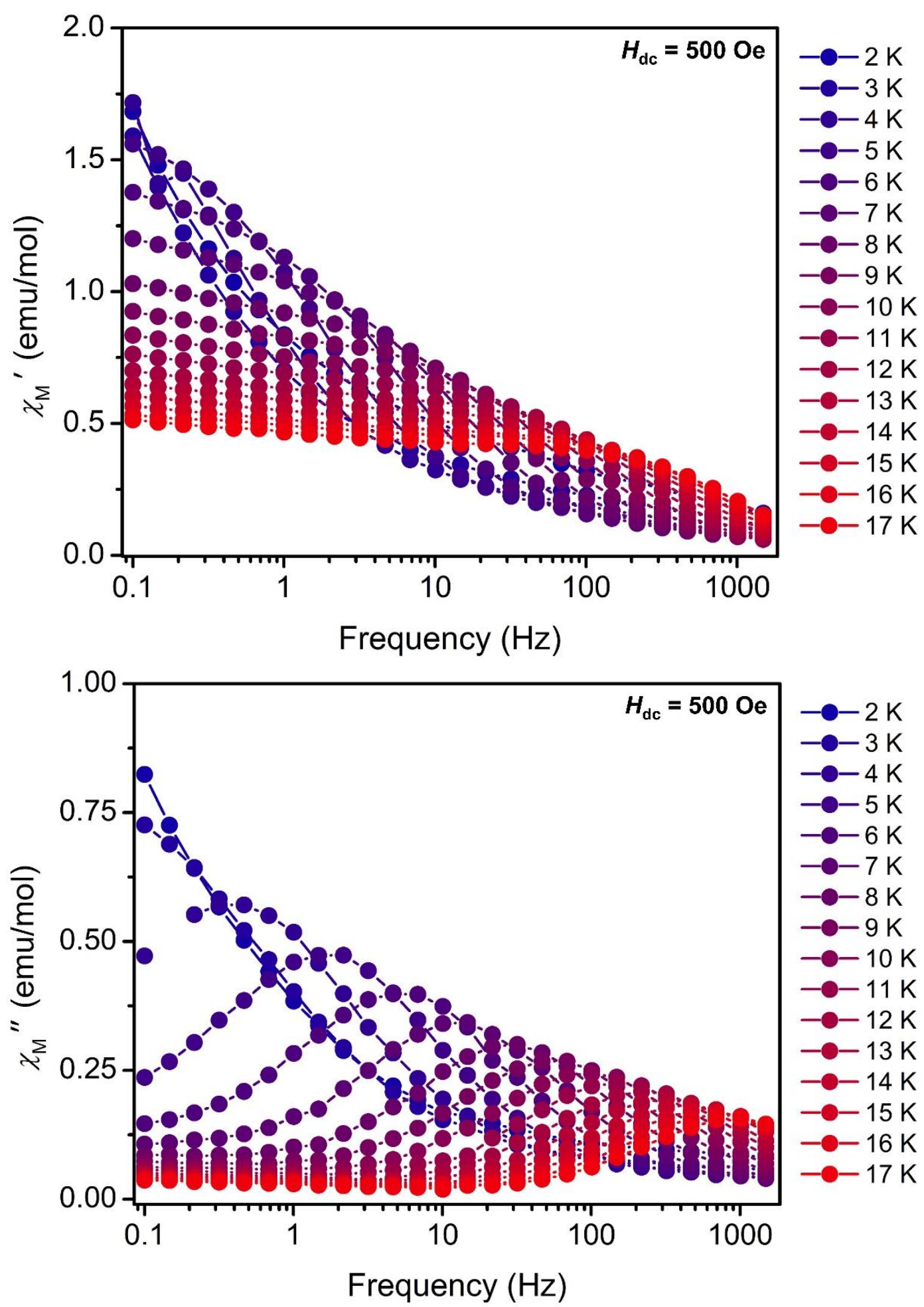

Figure S33. In-phase $\left(\chi_{\mathrm{M}^{\prime}}\right.$, top) and out-of-phase $\left(\chi_{\mathrm{M}}{ }^{\prime \prime}\right.$, bottom) components of the ac magnetic susceptibility for 3 under a 500 Oe applied dc field at frequencies ranging from $0.1-1500 \mathrm{~Hz}$ and temperatures from $2-17 \mathrm{~K}$ (1 K steps). The colored lines are guides for the eye. 


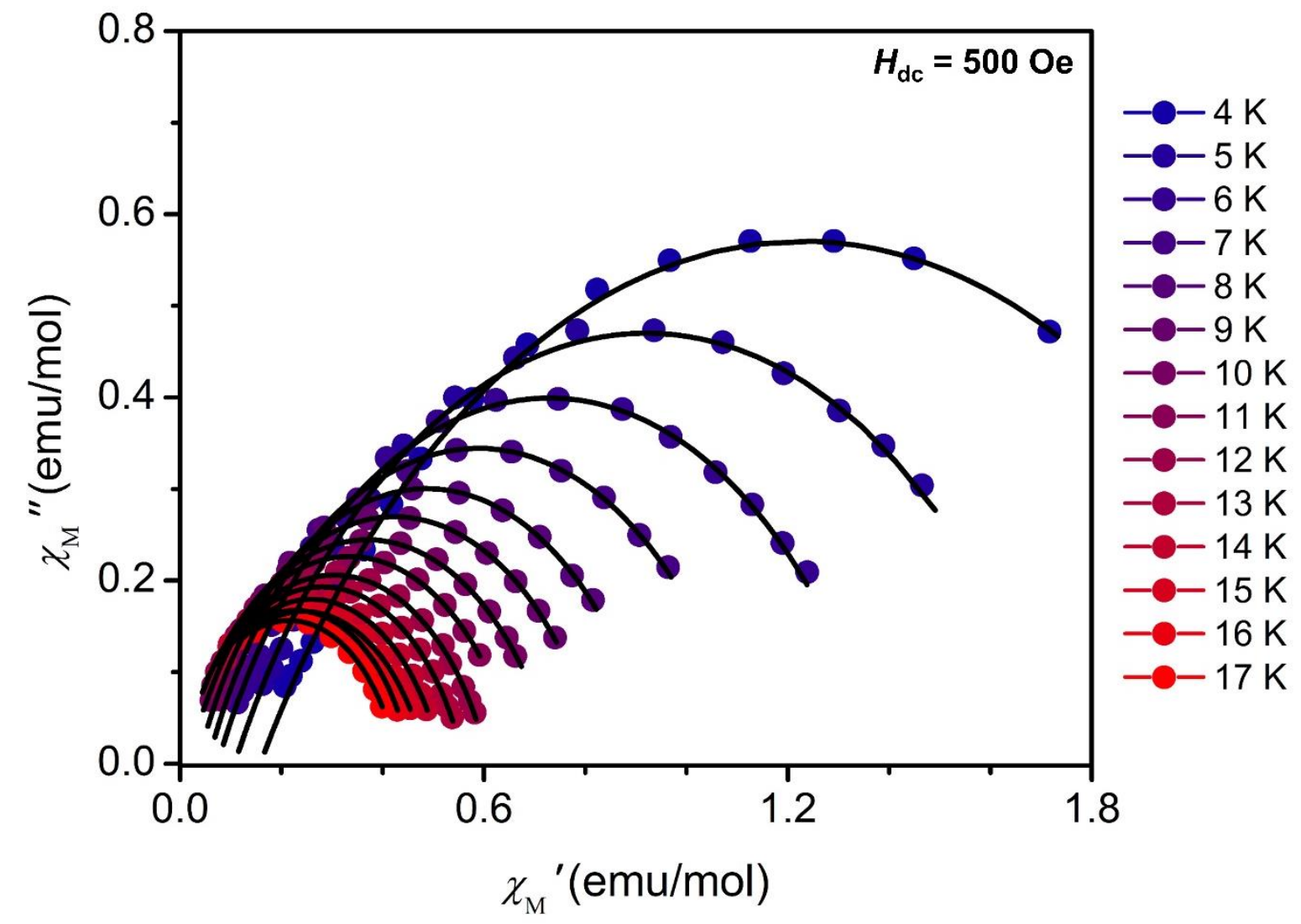

Figure S34. Cole-Cole plots for 3 from $4-17 \mathrm{~K}$ under a 500 Oe applied dc field (1 K steps). The black lines represent fits to the data using a generalized Debye model, which were used to extract $\tau$ values at each temperature. 

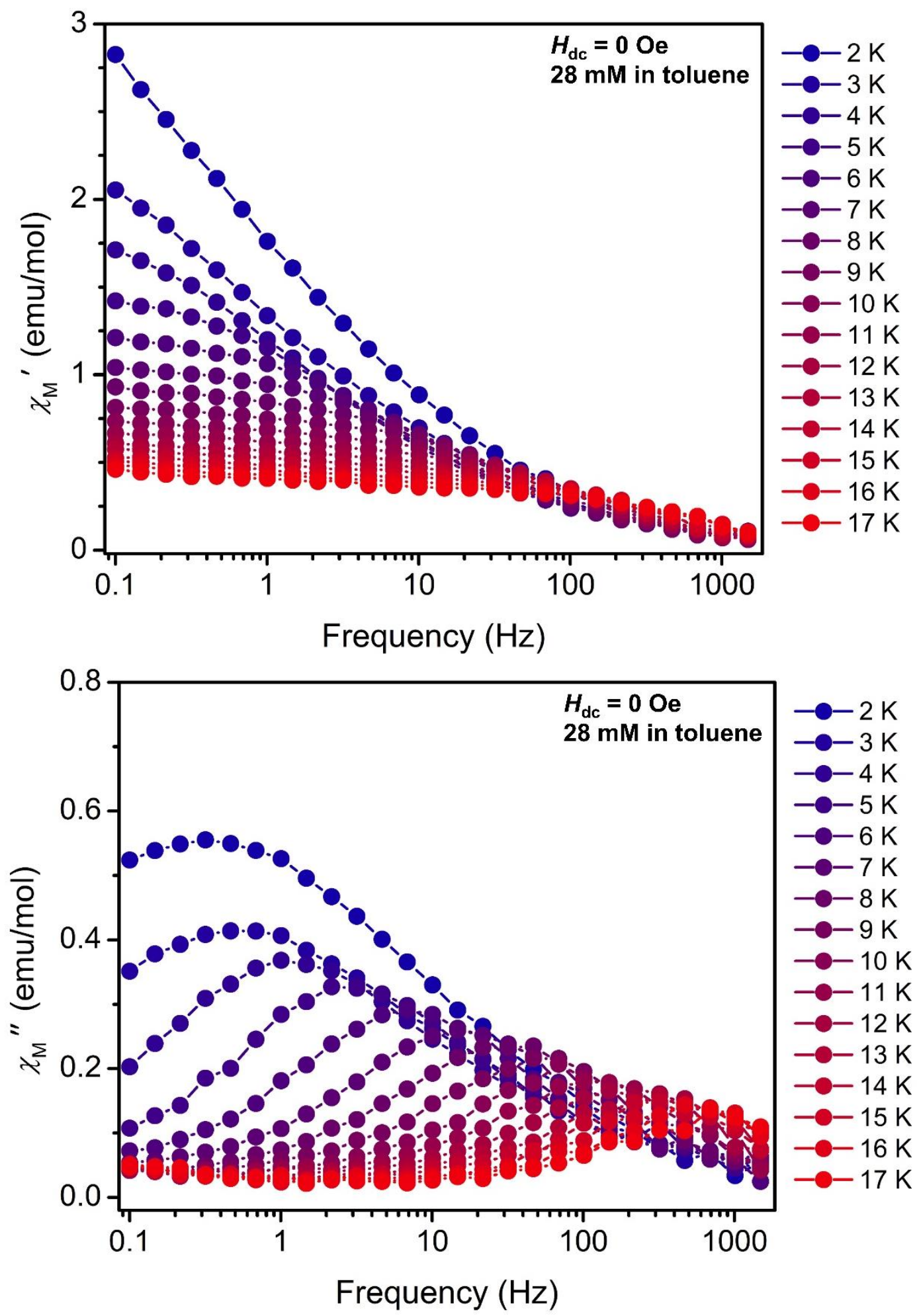

Figure S35. In-phase $\left(\chi_{\mathrm{M}^{\prime}}\right.$, top) and out-of-phase ( $\chi_{\mathrm{M}}{ }^{\prime \prime}$, bottom) components of the ac magnetic susceptibility for a $28 \mathrm{mM}$ solution of $\mathbf{3}$ in toluene under zero applied dc field at frequencies ranging from $0.1-1500 \mathrm{~Hz}$ and temperatures from $2-17 \mathrm{~K}(1 \mathrm{~K}$ steps $)$. The colored lines are guides for the eye. 


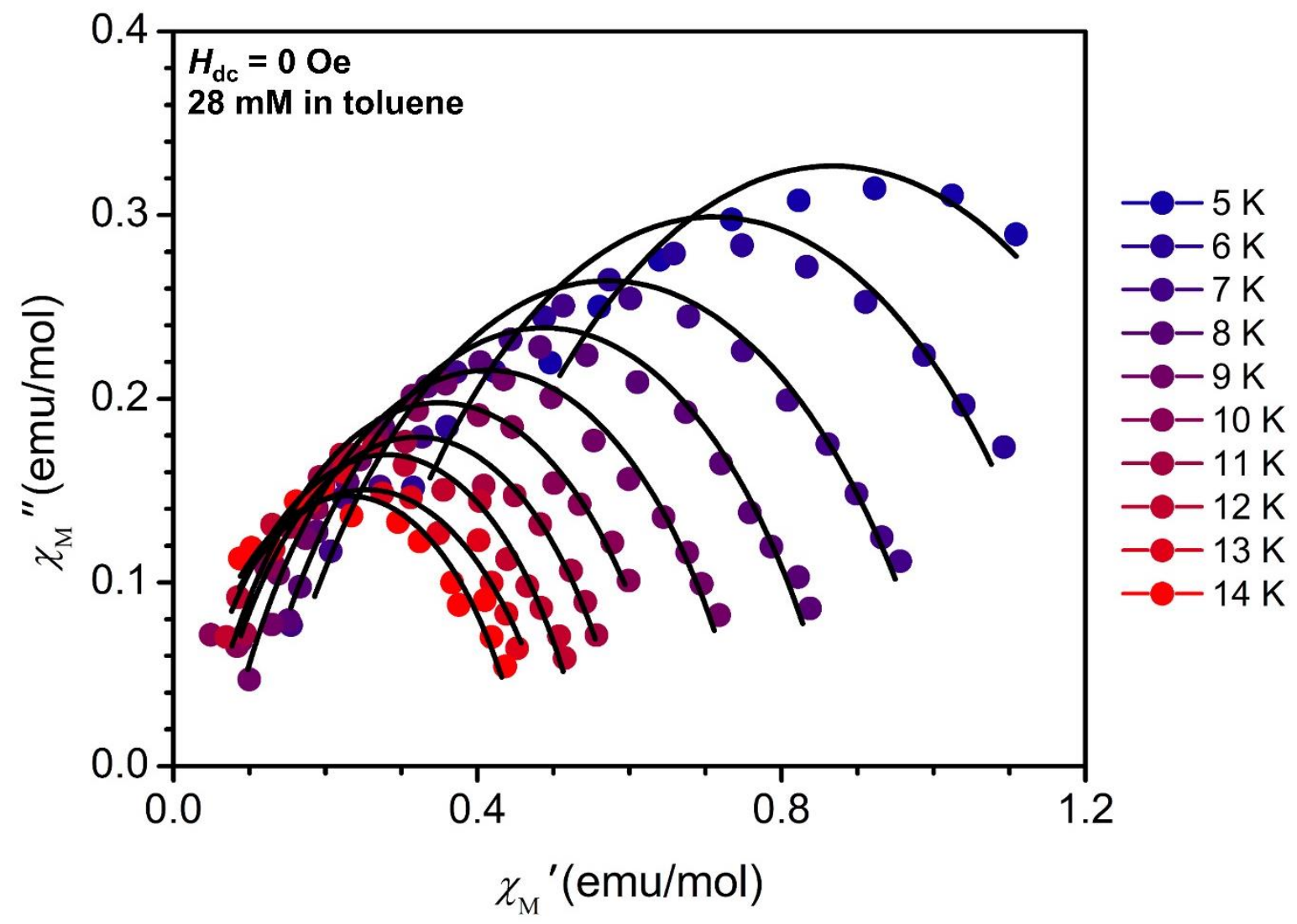

Figure S36. Cole-Cole plots for a $28 \mathrm{mM}$ solution of $\mathbf{3}$ in toluene from 5-14 K under zero applied dc field (1 K steps). The black lines represent fits to the data using a generalized Debye model, which were used to extract $\tau$ values at each temperature. 

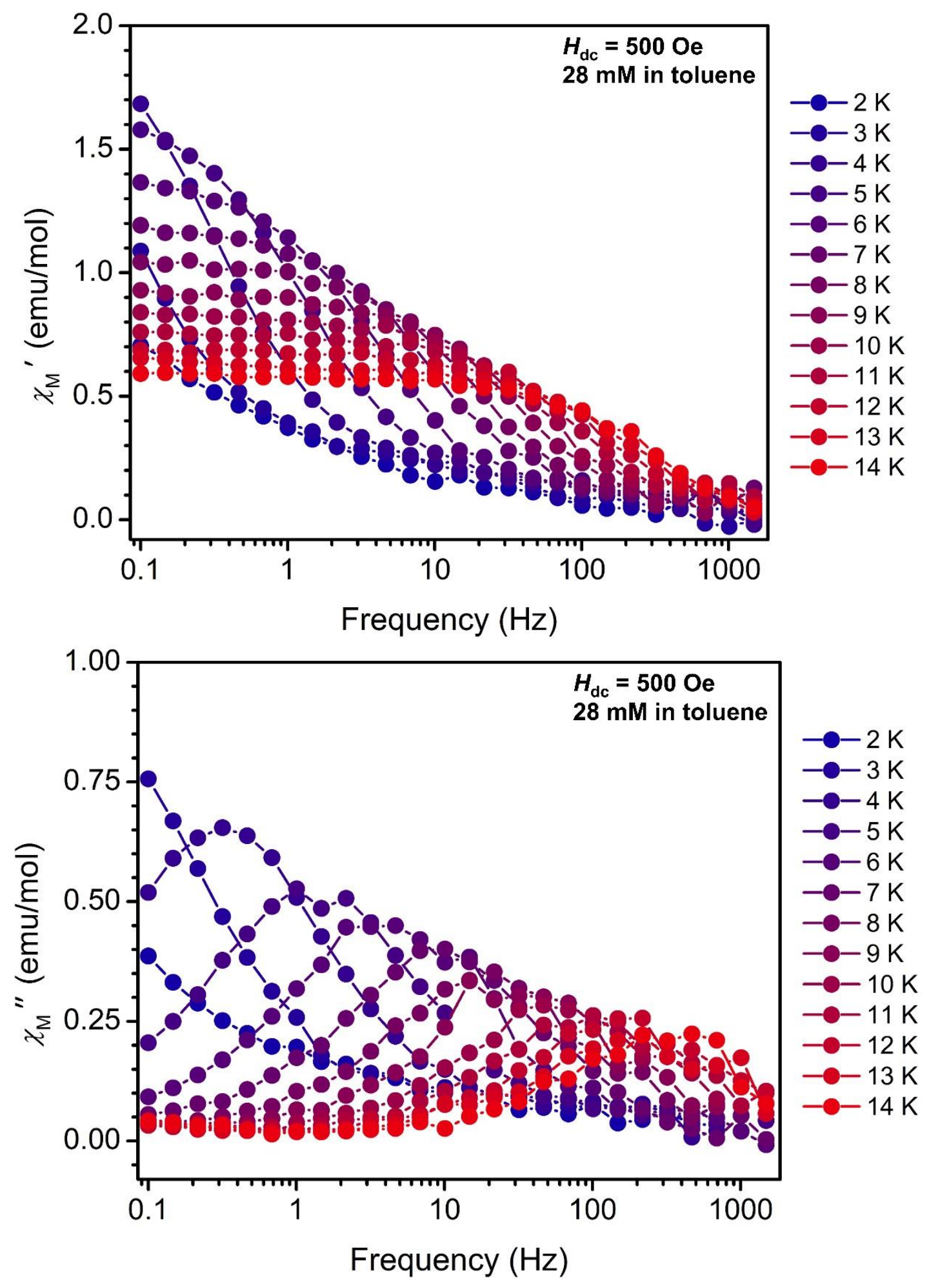

Figure S37. In-phase $\left(\chi_{\mathrm{M}}{ }^{\prime}\right.$, top) and out-of-phase ( $\chi_{\mathrm{M}}{ }^{\prime \prime}$, bottom) components of the ac magnetic susceptibility for a $28 \mathrm{mM}$ solution of $\mathbf{3}$ in toluene under a 500 Oe applied dc field at frequencies ranging from $0.1-1500 \mathrm{~Hz}$ and temperatures from $2-14 \mathrm{~K}(1 \mathrm{~K}$ steps $)$. The colored lines are guides for the eye. 


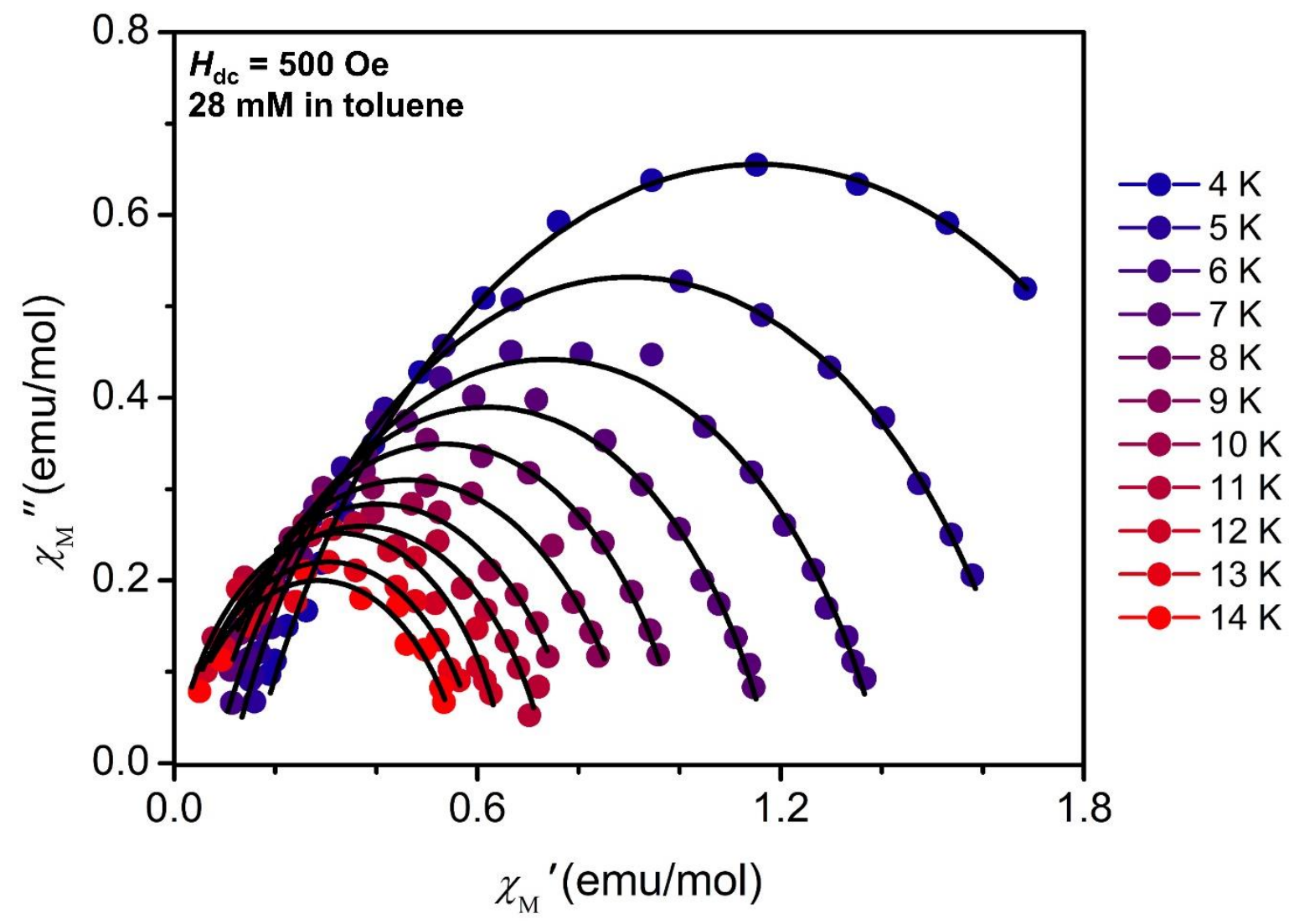

Figure S38. Cole-Cole plots for a $28 \mathrm{mM}$ solution of 3 in toluene from 4-14 K under a 500 Oe applied dc field (1 K steps). The black lines represent fits to the data using a generalized Debye model, which were used to extract $\tau$ values at each temperature. 
Dc Magnetic Relaxation Measurements. Dc magnetic relaxation measurements were collected by magnetizing a sample under an applied field of $5 \mathrm{~T}$, allowing 5 minutes for equilibration, and then subsequently returning the field to 0 Oe (no overshoot, hi-res disabled) or 500 Oe and measuring the magnetization at periodic time intervals. Dc magnetic relaxation data were fit to a stretched exponential function according to the equation:

$$
M(t)=M_{1}+\left(M_{0}-M_{1}\right)^{(t / \tau)^{n}}
$$

where $M(t)$ is the magnetization at time $t, M_{0}$ is the initial magnetization measured after the field has been removed, $M_{1}$ is the final value of the magnetization at $t=\infty, \tau$ is the magnetic relaxation time, and $n$ is a free variable. Though $M_{1}$ should equal zero at zero field, we found non-zero values for all dc magnetic relaxation measurements $T$ zero field, likely due to a small remnant field in the superconducting magnet. We therefore set $M_{1}$ equal to the value of the magnetization measured at the last time point of each experiment. At high temperatures, it was possible to measure the full decay of the magnetization within $1000 \mathrm{~s}$. At lower temperatures where magnetic relaxation slowed, experiments were conducted for $5000 \mathrm{~s}$. Even this length of time was not sufficient to observe the full decay of the magnetization for measurements of 2-3 at the lowest temperatures measured ( 2 to $20 \mathrm{~K}$ ), however, and therefore setting $M_{1}$ equal to the last value of the magnetization measured for these experiments represented an overestimate. Better fits to the data for these experiments were obtained by treating $M_{1}$ as a free variable $\left(M_{1}\right.$ values marked with an $*$ in the tables below).

In order to test the validity of the strategy, fits to higher temperature experiments with $M_{1}$ as a free variable were obtained and compared to previous fits. This resulted in values of $\tau$ within 5\% of those obtained for fits with $M_{1}$ equal to the last value of the magnetization measured in the experiment. Furthermore, the values obtained for $\tau$ with $M_{1}$ as a free variable were consistently lower than those obtained with $M_{1}$ equal to the last value of the magnetization, demonstrating that this alternative fitting strategy did not overestimate the value of $\tau$ at low temperatures.

For dc relaxation measurements at 500 Oe, field-cooled susceptibility measurements were collected at 500 Oe and these values were used for $M_{1}$. 
Table S3. Parameters used to fit dc magnetic relaxation data for $\mathbf{2}$ at zero applied field and magnetic relaxation times extracted from these fits.

\begin{tabular}{ccccc}
\hline $\boldsymbol{T}(\mathbf{K})$ & $\boldsymbol{M}_{\mathbf{0}}\left(\boldsymbol{\mu}_{\mathbf{b}}\right)$ & $\boldsymbol{M}_{\mathbf{1}}\left(\boldsymbol{\mu}_{\mathbf{b}}\right)$ & $\boldsymbol{n}$ & $\boldsymbol{\tau}(\mathbf{s})^{\mathrm{a}}$ \\
\hline 2 & 0.42546 & $0.04007^{*}$ & 0.644 & 569.527 \\
5 & 0.42881 & $0.04074^{*}$ & 0.661 & 611.137 \\
10 & 0.43225 & $0.03625^{*}$ & 0.635 & 630.158 \\
15 & 0.33748 & $0.02633^{*}$ & 0.696 & 781.132 \\
20 & 0.32891 & $0.03096^{*}$ & 0.711 & 645.590 \\
25 & 0.28351 & $0.02015^{*}$ & 0.725 & 642.143 \\
30 & 0.23763 & $0.01244^{*}$ & 0.749 & 609.369 \\
35 & 0.19042 & $0.00541^{*}$ & 0.775 & 559.920 \\
40 & 0.14173 & 0.00131 & 0.804 & 432.189 \\
45 & 0.08062 & 0.00680 & 0.881 & 297.966 \\
50 & 0.02577 & 0.00021 & 0.934 & 142.592 \\
55 & 0.00348 & 0.00017 & 0.910 & 62.242 \\
\hline
\end{tabular}

${ }^{a}$ Values for $\tau$ are likely only accurate to three significant figures. The full value extracted from the fit is reported for reproducibility.

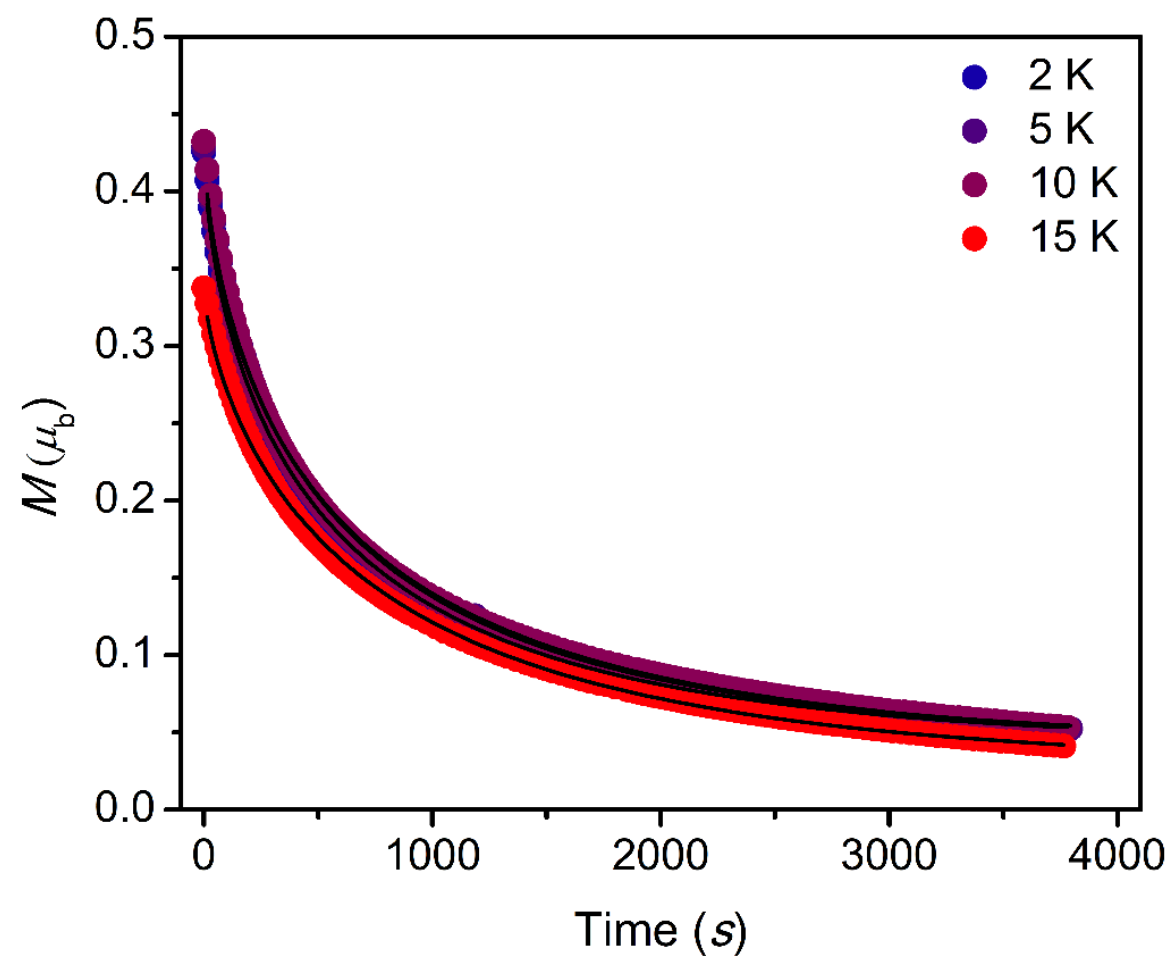

Figure S39. De magnetic relaxation data for 2 collected at 2, 5, 10, and $15 \mathrm{~K}$ and under zero applied dc field. Black lines represent the fits to the data using stretched exponential functions, which were used to extract $\tau$. 


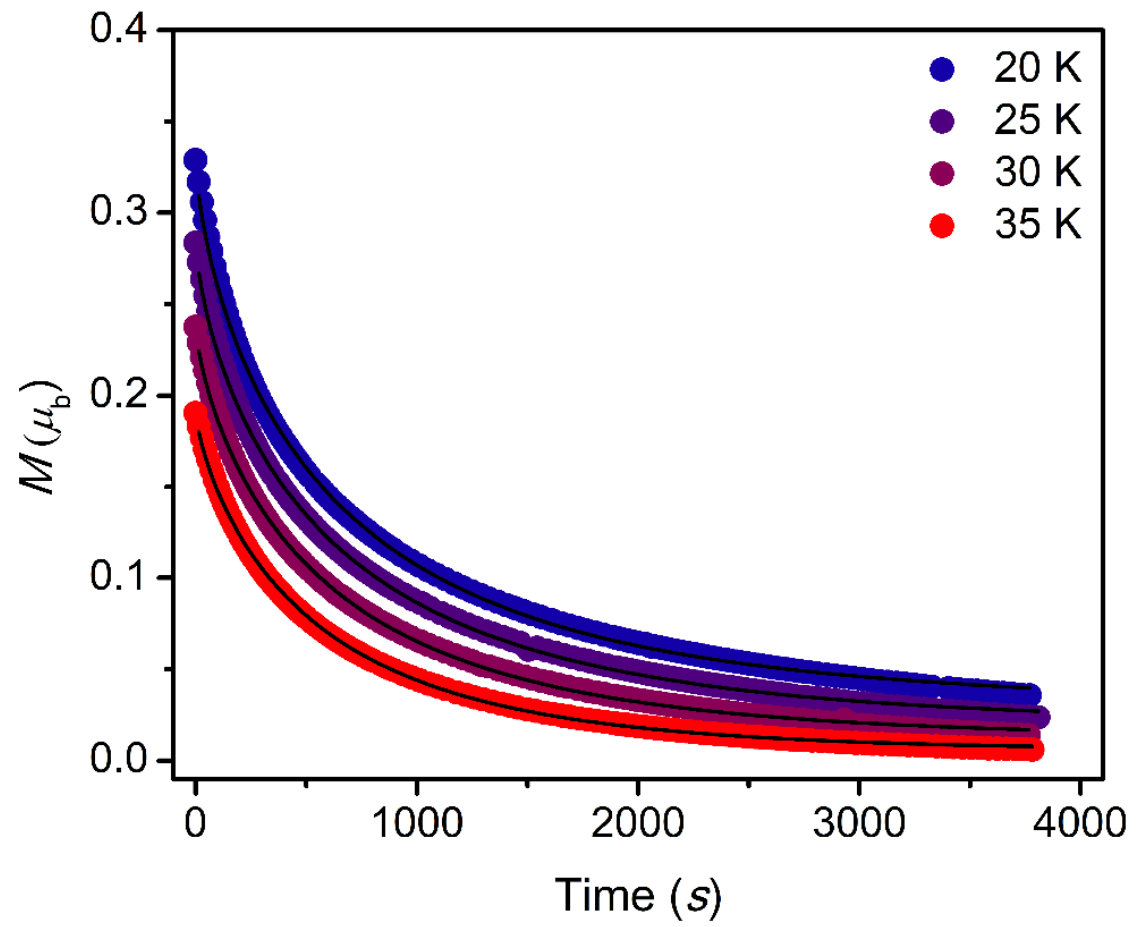

Figure S40. Dc magnetic relaxation data for 2 collected at 20, 25, 30, and $35 \mathrm{~K}$ and under zero applied dc field. Black lines represent the fits to the data using stretched exponential functions, which were used to extract $\tau$.

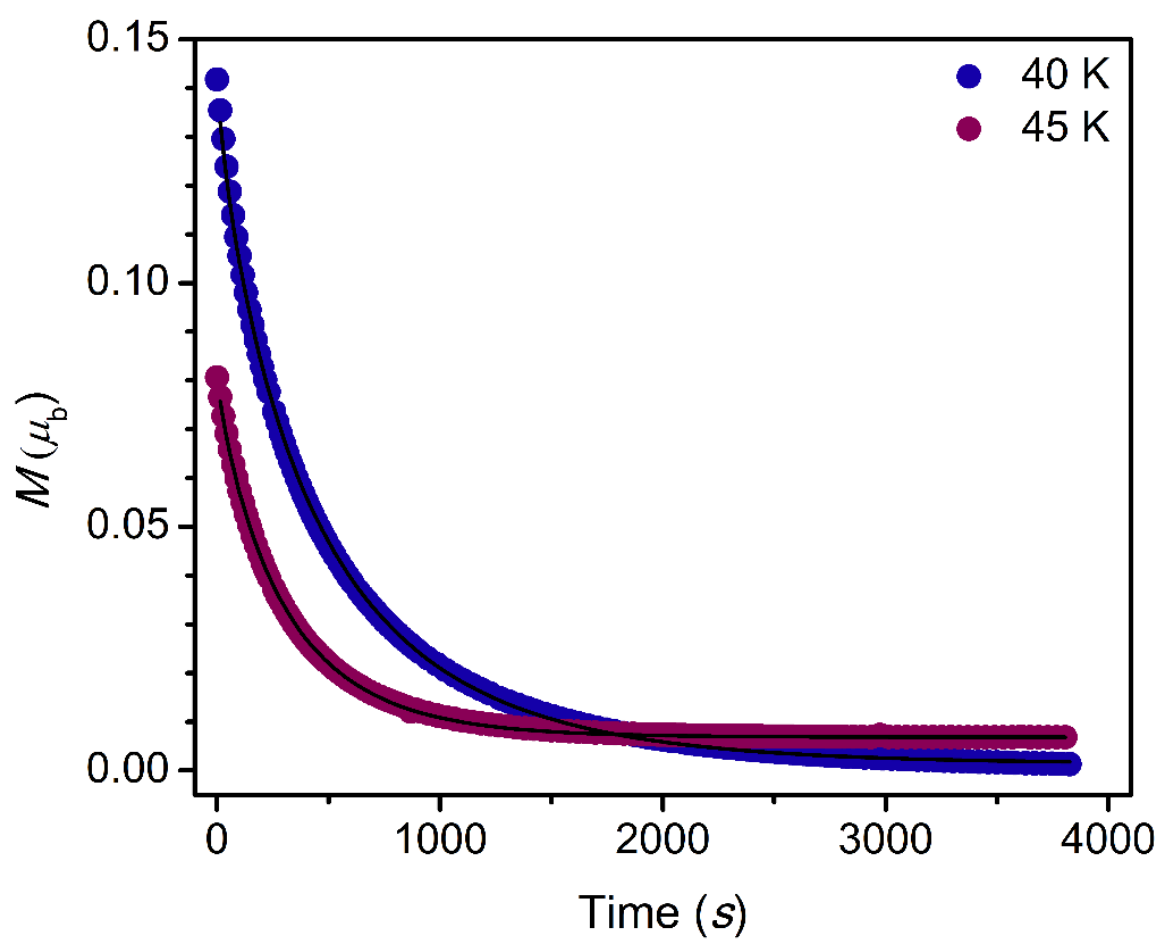

Figure S41. Dc magnetic relaxation data for 2 collected at 40 and $45 \mathrm{~K}$ and under zero applied dc field. Black lines represent the fits to the data using stretched exponential functions, which were used to extract $\tau$. 


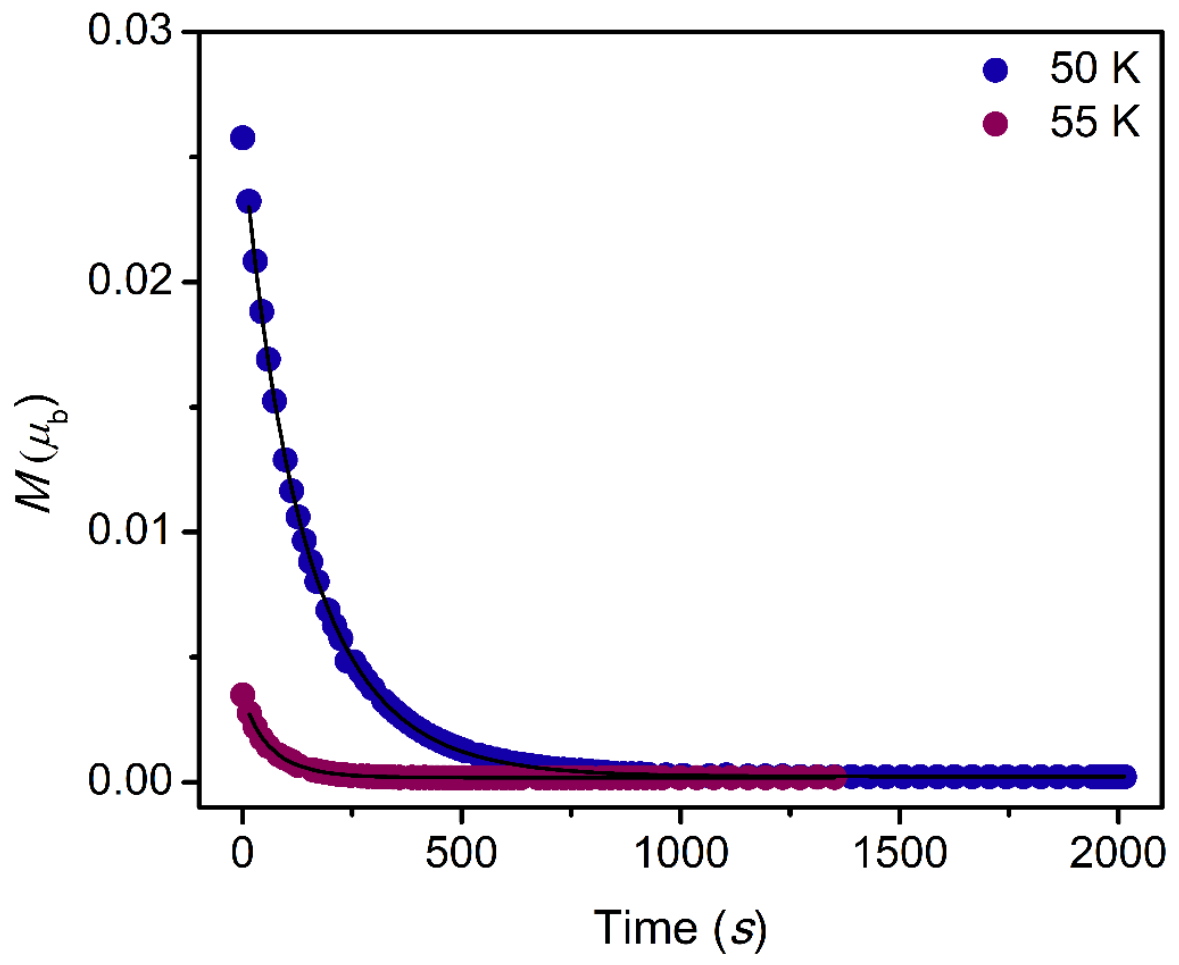

Figure S42. Dc magnetic relaxation data for 2 collected at 50 and $55 \mathrm{~K}$ and under zero applied dc field. Black lines represent the fits to the data using stretched exponential functions, which were used to extract $\tau$. 
Table S4. Parameters used to fit dc magnetic relaxation data for 2 at 500 Oe and magnetic relaxation times extracted from these fits.

\begin{tabular}{ccccc}
\hline $\boldsymbol{T}(\mathbf{K})$ & $\boldsymbol{M}_{\mathbf{0}}\left(\boldsymbol{\mu}_{\mathbf{b}}\right)$ & $\boldsymbol{M}_{\mathbf{1}}\left(\boldsymbol{\mu}_{\mathbf{b}}\right)$ & $\boldsymbol{n}$ & $\boldsymbol{\tau}(\mathbf{s})^{\mathrm{a}}$ \\
\hline 2 & 0.91201 & $0.46652^{*}$ & 0.565 & 794.888 \\
5 & 0.68669 & 0.20796 & 0.597 & 794.888 \\
10 & 0.60115 & 0.11227 & 0.586 & 794.888 \\
15 & 0.52809 & 0.08374 & 0.660 & 794.888 \\
20 & 0.45144 & 0.06563 & 0.642 & 795.371 \\
25 & 0.38208 & 0.05390 & 0.670 & 719.045 \\
30 & 0.31331 & 0.04526 & 0.717 & 653.577 \\
35 & 0.24366 & 0.03830 & 0.780 & 568.018 \\
40 & 0.17834 & 0.03239 & 0.858 & 419.568 \\
45 & 0.10482 & 0.02407 & 0.879 & 280.659 \\
50 & 0.04586 & 0.02159 & 0.879 & 138.179 \\
\hline
\end{tabular}

${ }^{a}$ Values for $\tau$ are likely only accurate to three significant figures. The full value extracted from the fit is reported for reproducibility.

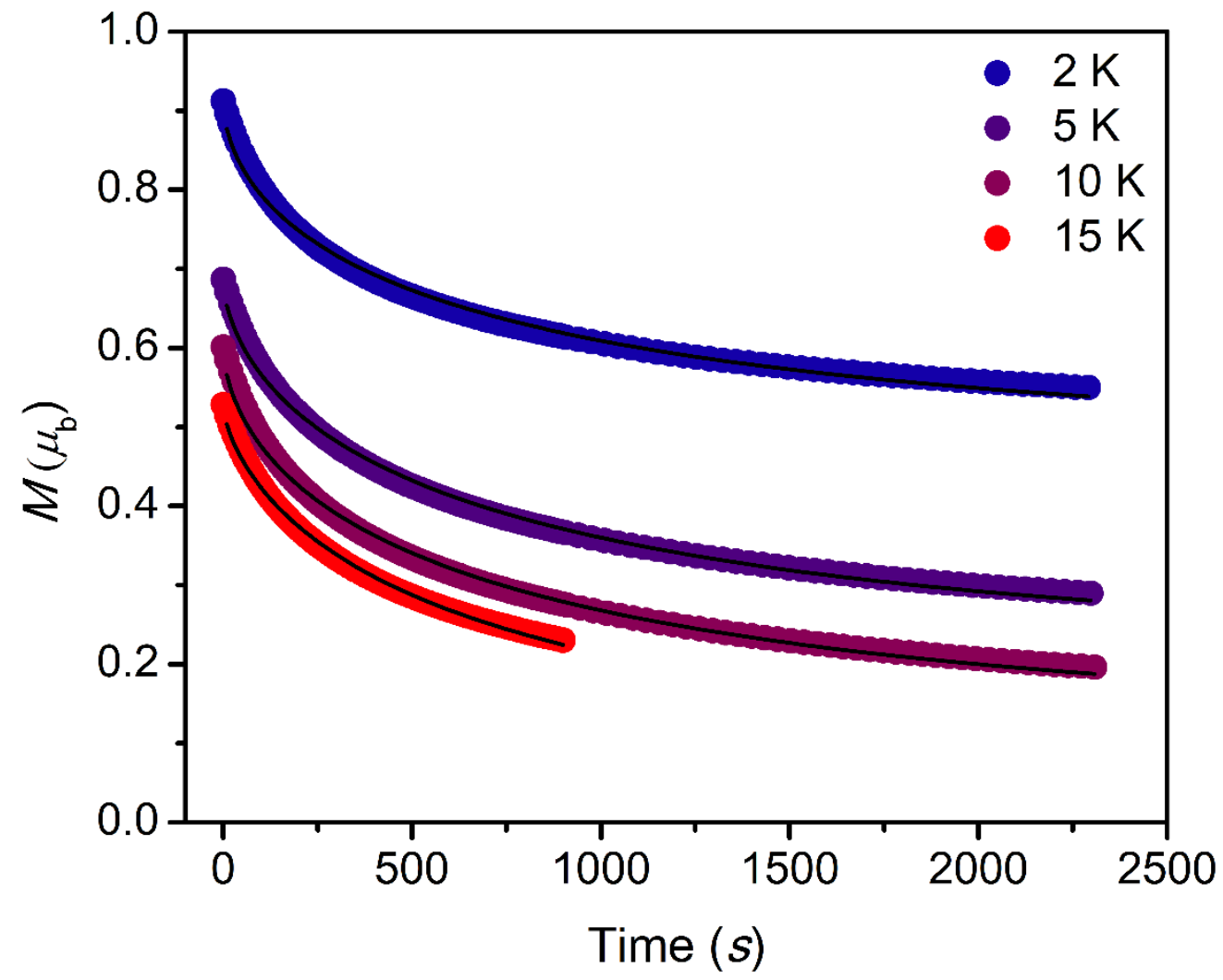

Figure S43. Dc magnetic relaxation data for 2 collected at 2, 5, 10, and $15 \mathrm{~K}$ and under an applied dc field of 500 Oe. Black lines represent the fits to the data using stretched exponential functions, which were used to extract $\tau$. 


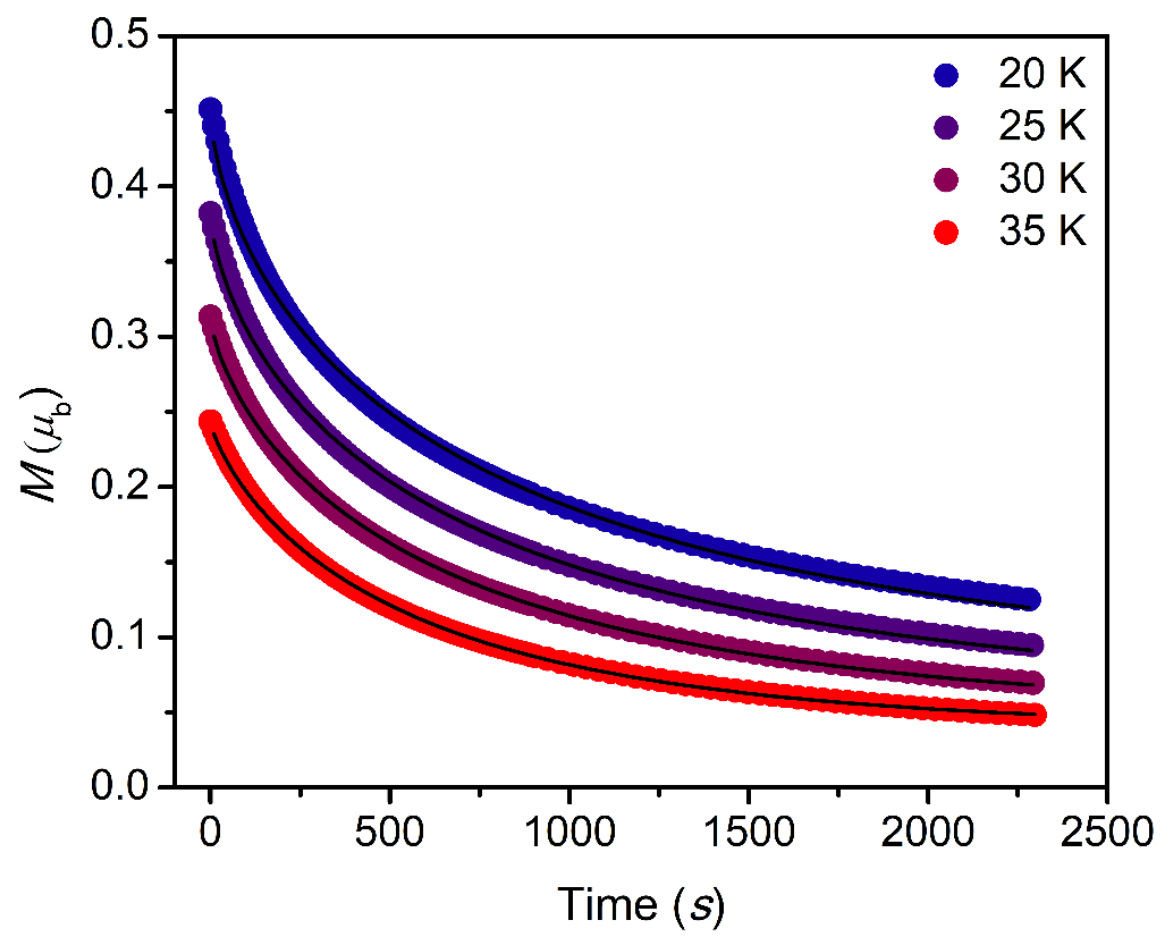

Figure S44. De magnetic relaxation data for 2 collected at 20, 25, 30, and $35 \mathrm{~K}$ and under an applied dc field of 500 Oe. Black lines represent the fits to the data using stretched exponential functions, which were used to extract $\tau$.

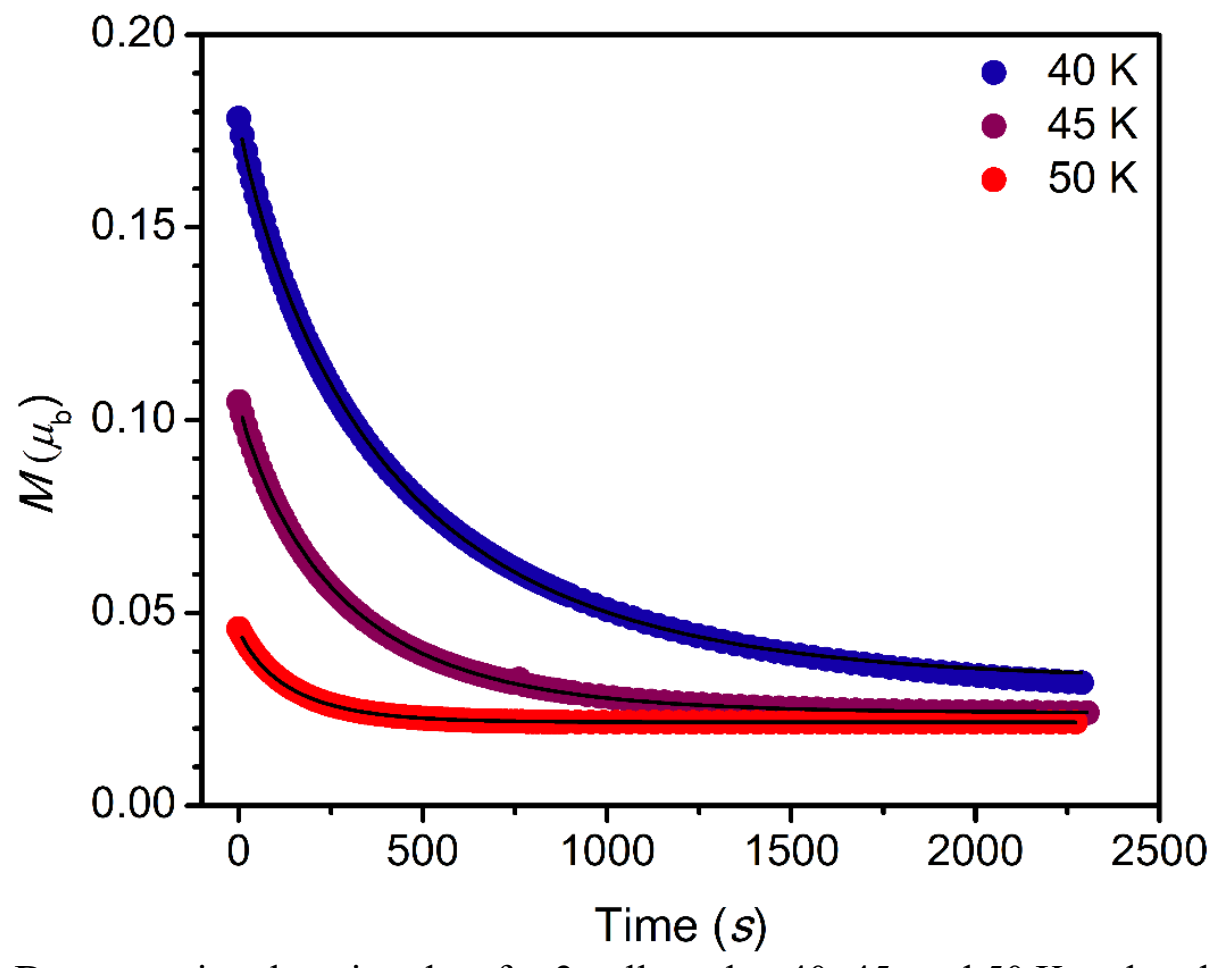

Figure S45. Dc magnetic relaxation data for 2 collected at 40, 45, and $50 \mathrm{~K}$ and under an applied dc field of 500 Oe. Black lines represent the fits to the data using stretched exponential functions, which were used to extract $\tau$. 
Table S5. Parameters used to fit dc magnetic relaxation data for a $19 \mathrm{mM}$ solution of 2 in toluene at zero applied field and magnetic relaxation times extracted from these fits.

\begin{tabular}{ccccc}
\hline $\boldsymbol{T}(\mathbf{K})$ & $\boldsymbol{M}_{\mathbf{0}}\left(\boldsymbol{\mu}_{\mathbf{b}}\right)$ & $\boldsymbol{M}_{\mathbf{1}}\left(\boldsymbol{\mu}_{\mathbf{b}}\right)$ & $\boldsymbol{n}$ & $\boldsymbol{\tau}(\mathbf{s})^{\mathrm{a}}$ \\
\hline 2 & 0.13301 & $0.10975^{*}$ & 0.510 & 695.989 \\
5 & 0.04169 & $0.00920^{*}$ & 0.571 & 238.122 \\
10 & 0.03652 & $0.00801^{*}$ & 0.558 & 269.979 \\
15 & 0.03775 & $0.00675^{*}$ & 0.506 & 228.571 \\
20 & 0.03281 & $0.00520^{*}$ & 0.542 & 280.109 \\
25 & 0.02911 & $0.00320^{*}$ & 0.548 & 297.812 \\
30 & 0.02288 & $0.00181^{*}$ & 0.637 & 357.986 \\
35 & 0.02502 & $0.00069^{*}$ & 0.526 & 242.411 \\
40 & 0.01705 & 0.00036 & 0.695 & 239.050 \\
45 & 0.01270 & 0.00027 & 0.734 & 142.756 \\
50 & 0.00500 & 0.00023 & 1.000 & 99.098 \\
\hline
\end{tabular}

${ }^{a}$ Values for $\tau$ are likely only accurate to three significant figures. The full value extracted from the fit is reported for reproducibility.

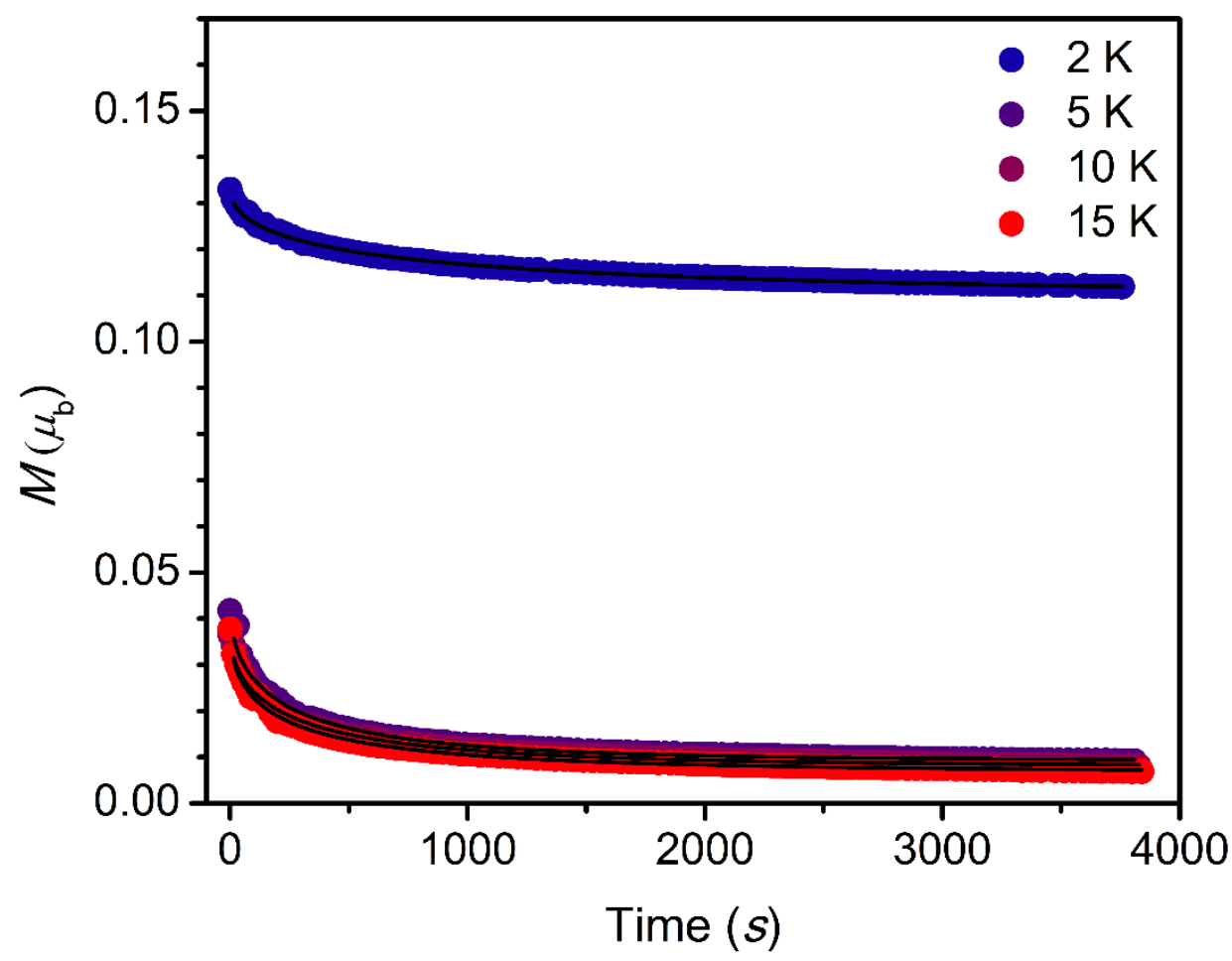

Figure S46. Dc magnetic relaxation data for a $19 \mathrm{mM}$ solution of 2 in toluene collected at 2, 5, 10 , and $15 \mathrm{~K}$ and under zero applied dc field. Black lines represent the fits to the data using stretched exponential functions, which were used to extract $\tau$. 


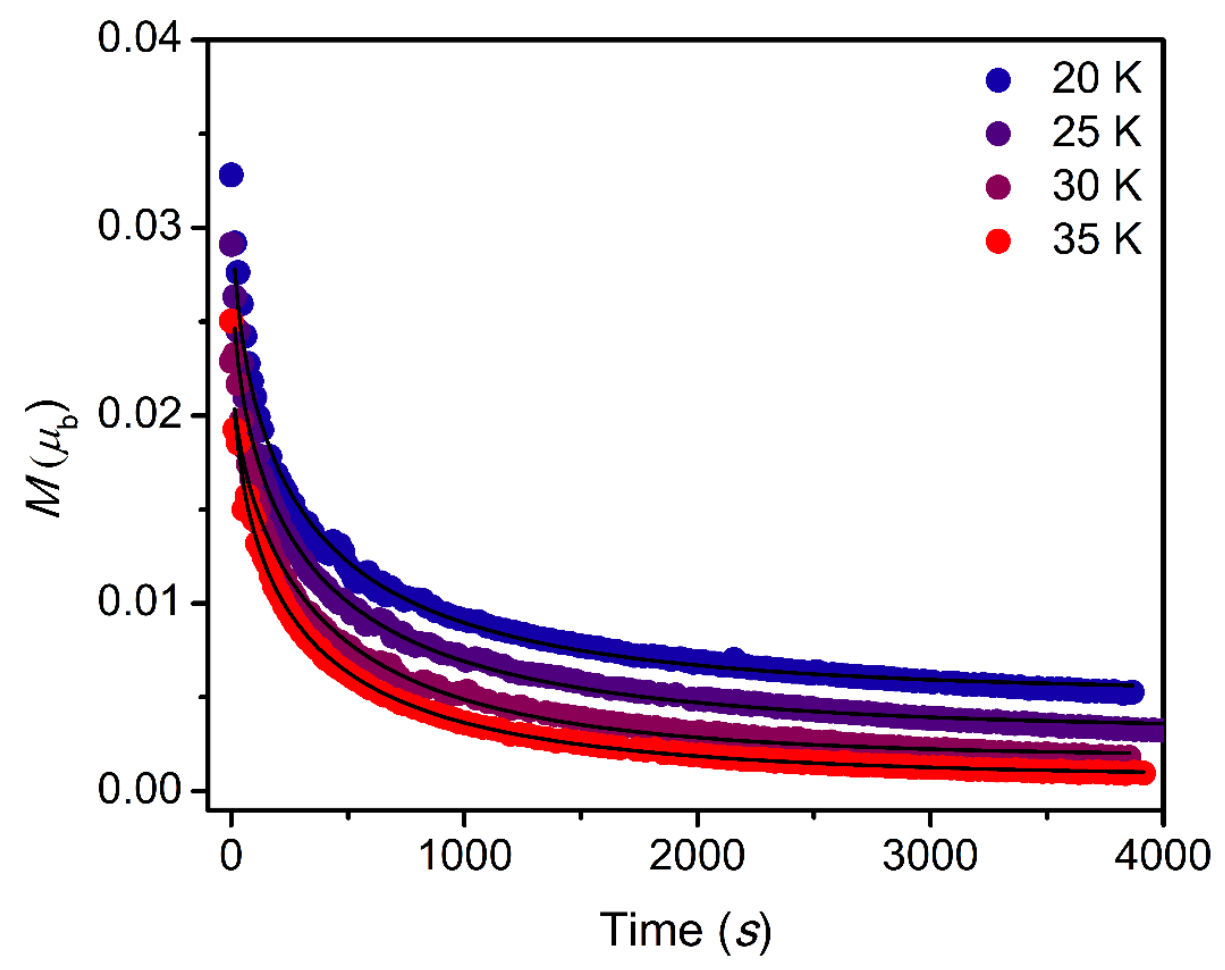

Figure S47. Dc magnetic relaxation data for a $19 \mathrm{mM}$ solution of $\mathbf{2}$ in toluene collected at 20, 25, 30 , and $35 \mathrm{~K}$ and under zero applied dc field. Black lines represent the fits to the data using stretched exponential functions, which were used to extract $\tau$.

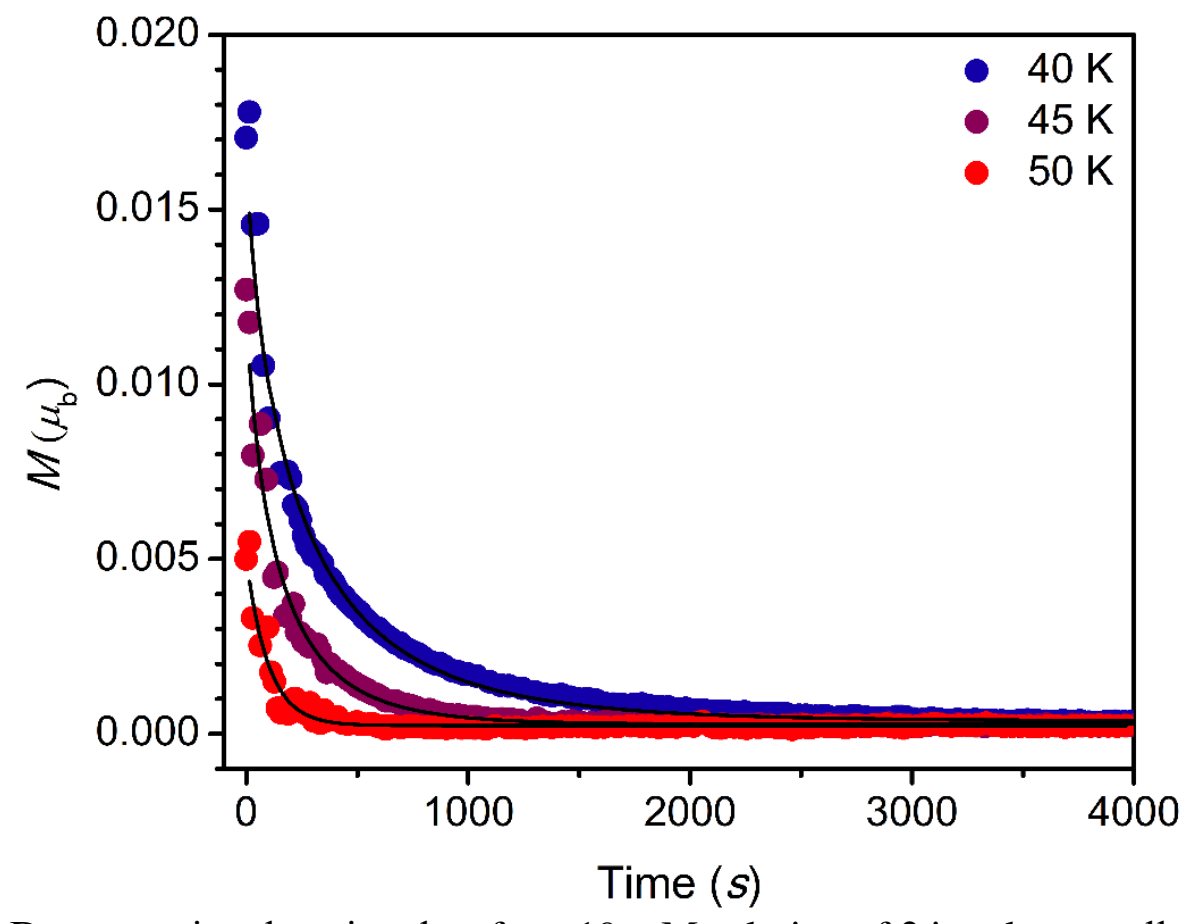

Figure S48. Dc magnetic relaxation data for a $19 \mathrm{mM}$ solution of $\mathbf{2}$ in toluene collected at 40, 45, and $50 \mathrm{~K}$ and under zero applied dc field. Black lines represent the fits to the data using stretched exponential functions, which were used to extract $\tau$. 
Table S6. Parameters used to fit dc magnetic relaxation data for a $19 \mathrm{mM}$ solution of $\mathbf{2}$ in toluene at 500 Oe and magnetic relaxation times extracted from these fits.

\begin{tabular}{ccccc}
\hline $\boldsymbol{T}(\mathbf{K})$ & $\boldsymbol{M}_{\mathbf{0}}\left(\boldsymbol{\mu}_{\mathbf{b}}\right)$ & $\boldsymbol{M}_{\mathbf{1}}\left(\boldsymbol{\mu}_{\mathbf{b}}\right)$ & $\boldsymbol{n}$ & $\boldsymbol{\tau}(\mathbf{s})^{\mathrm{a}}$ \\
\hline 2 & 0.53515 & 0.40510 & 0.360 & 3542479.980 \\
5 & 0.33221 & 0.17403 & 0.366 & 1508689.837 \\
10 & 0.25195 & 0.09128 & 0.479 & 202520.071 \\
15 & 0.21301 & 0.06075 & 0.586 & 47303.034 \\
20 & 0.18323 & 0.04602 & 0.647 & 19883.087 \\
25 & 0.15536 & 0.03706 & 0.724 & 7910.162 \\
30 & 0.13101 & 0.03091 & 0.758 & 3844.352 \\
35 & 0.10878 & 0.02646 & 0.832 & 1902.502 \\
40 & 0.08491 & 0.02305 & 0.870 & 904.773 \\
45 & 0.05737 & 0.02029 & 0.910 & 402.213 \\
50 & 0.03051 & 0.01784 & 0.958 & 182.543 \\
\hline
\end{tabular}

${ }^{a}$ Values for $\tau$ are likely only accurate to three significant figures. The full value extracted from the fit is reported for reproducibility.

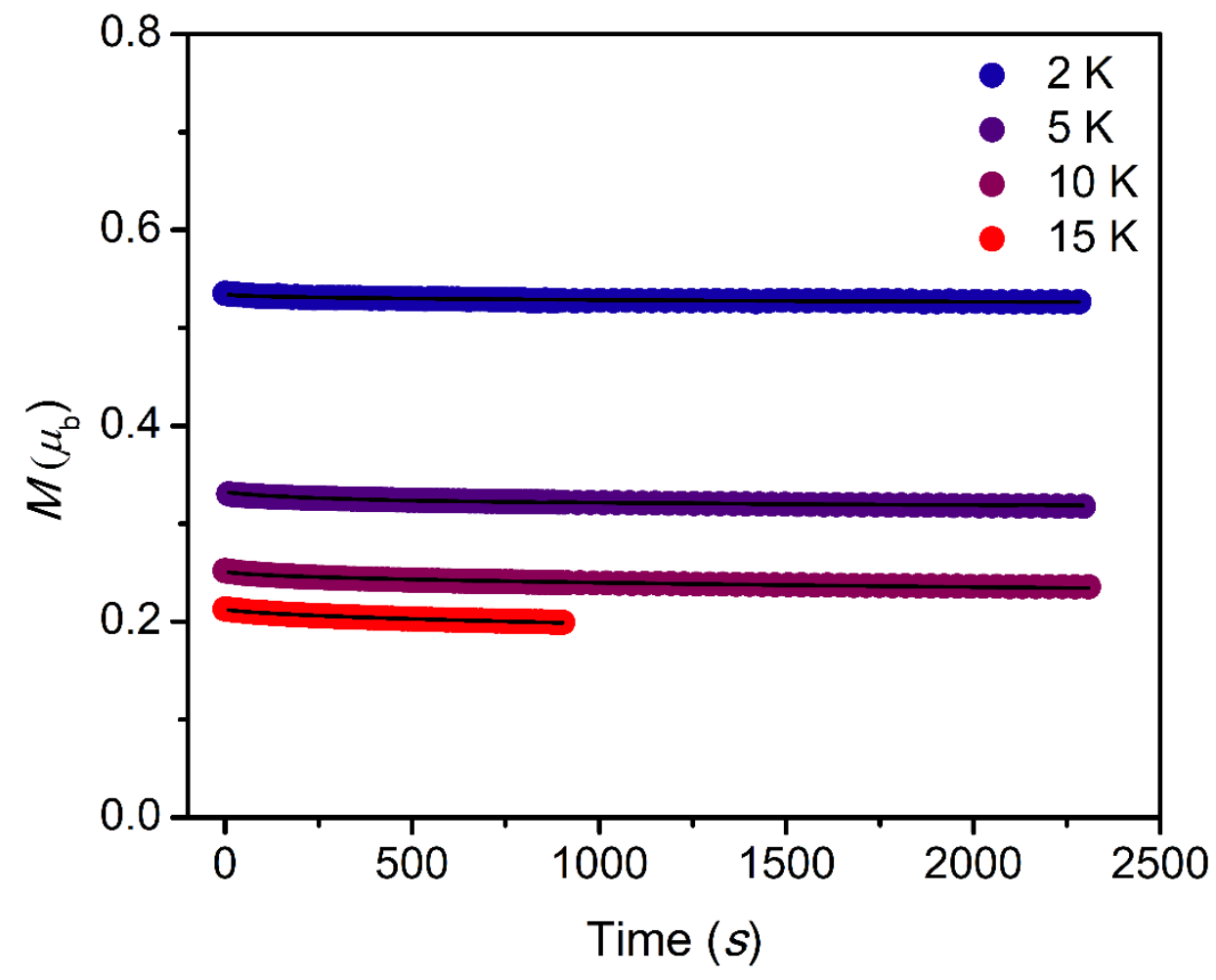

Figure S49. Dc magnetic relaxation data for a $19 \mathrm{mM}$ solution of $\mathbf{2}$ in toluene collected at 2, 5, 10 , and $15 \mathrm{~K}$ and under an applied dc field of 500 Oe. Black lines represent the fits to the data using stretched exponential functions, which were used to extract $\tau$. 


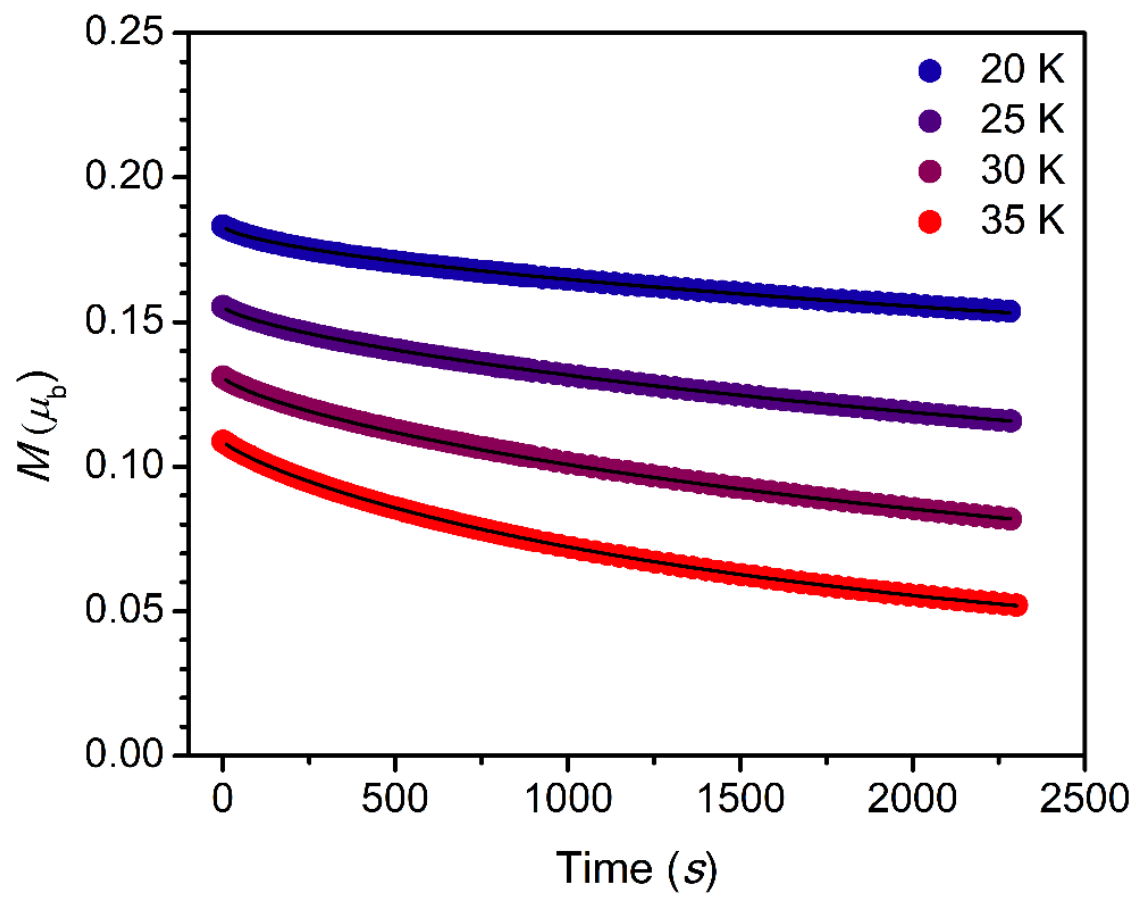

Figure S50. Dc magnetic relaxation data for a $19 \mathrm{mM}$ solution of $\mathbf{2}$ in toluene collected at 20, 25, 30 , and $35 \mathrm{~K}$ and under an applied dc field of 500 Oe. Black lines represent the fits to the data using stretched exponential functions, which were used to extract $\tau$.

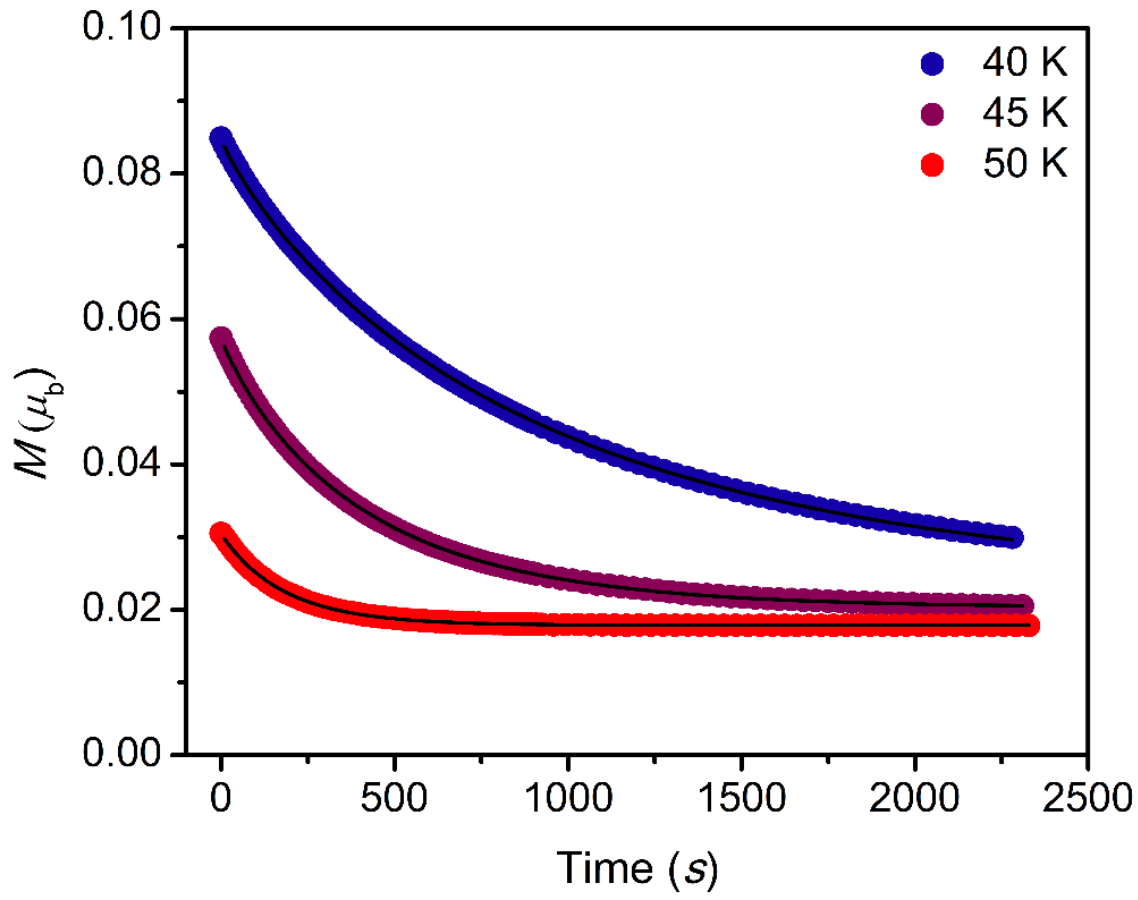

Figure S51. Dc magnetic relaxation data for a $19 \mathrm{mM}$ solution of 2 in toluene collected at 40, 45, and $50 \mathrm{~K}$ and under an applied dc field of 500 Oe. Black lines represent the fits to the data using stretched exponential functions, which were used to extract $\tau$. 
Table S7. Parameters used to fit dc magnetic relaxation data for 3 at 500 Oe and magnetic relaxation times extracted from these fits.

\begin{tabular}{ccccc}
\hline $\boldsymbol{T}(\mathbf{K})$ & $\boldsymbol{M}_{\mathbf{0}}\left(\boldsymbol{\mu}_{\mathbf{b}}\right)$ & $\boldsymbol{M}_{\mathbf{1}}\left(\boldsymbol{\mu}_{\mathbf{b}}\right)$ & $\boldsymbol{n}$ & $\boldsymbol{\tau}(\mathbf{s})^{\mathrm{a}}$ \\
\hline 2 & 0.75484 & 0.60027 & 0.531 & 223.793 \\
5 & 0.33039 & 0.24931 & 0.669 & 223.882 \\
10 & 0.19735 & 0.12884 & 0.656 & 223.864 \\
15 & 0.15168 & 0.08660 & 0.678 & 224.727 \\
20 & 0.12399 & 0.06539 & 0.664 & 229.226 \\
25 & 0.10147 & 0.05163 & 0.687 & 213.936 \\
30 & 0.08415 & 0.04264 & 0.700 & 194.638 \\
35 & 0.06979 & 0.03632 & 0.717 & 184.311 \\
40 & 0.05878 & 0.03160 & 0.732 & 171.191 \\
45 & 0.04967 & 0.02808 & 0.730 & 161.758 \\
50 & 0.04237 & 0.02529 & 0.764 & 148.359 \\
\hline
\end{tabular}

${ }^{a}$ Values for $\tau$ are likely only accurate to three significant figures. The full value extracted from the fit is reported for reproducibility.

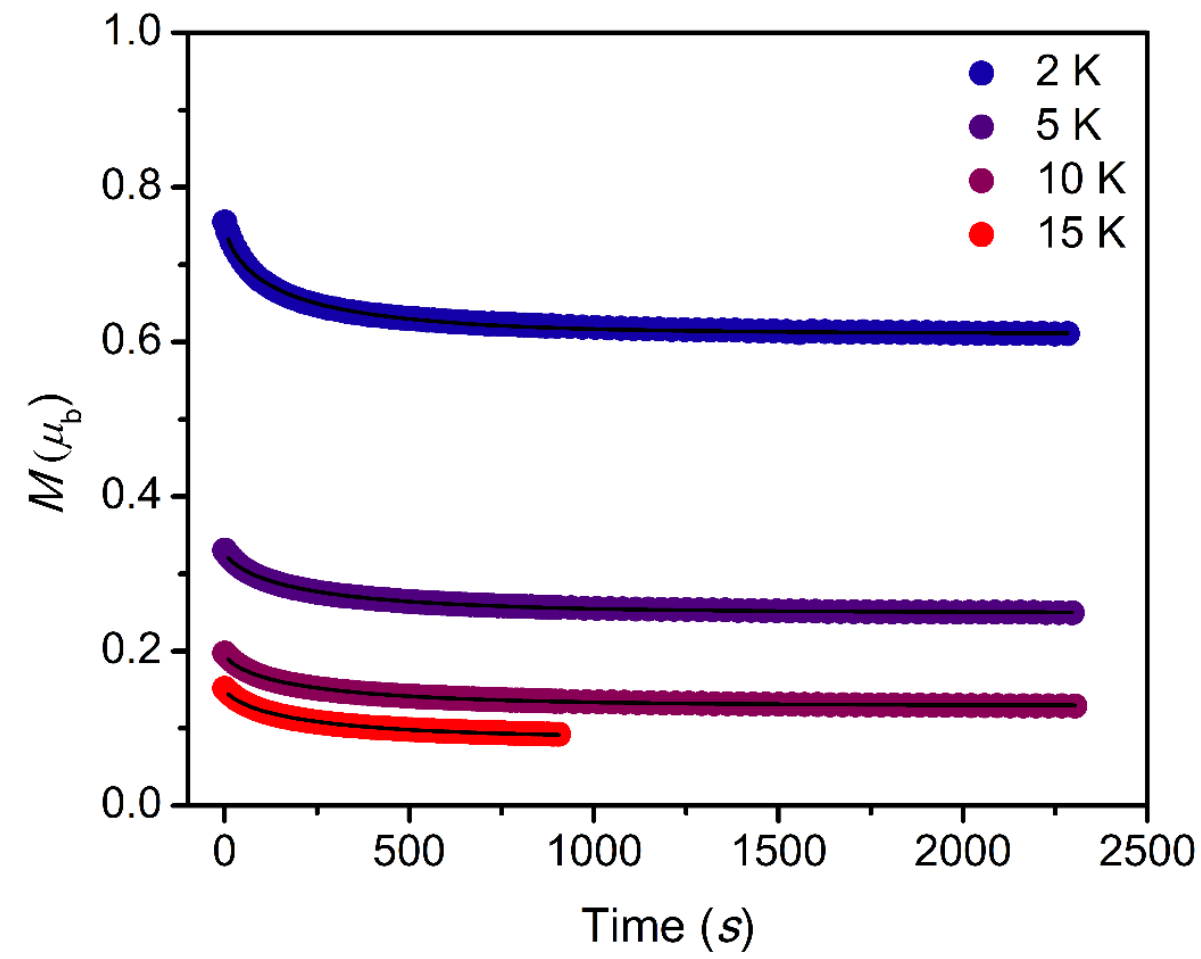

Figure S52. Dc magnetic relaxation data for 3 collected at 2, 5, 10, and $15 \mathrm{~K}$ and under an applied dc field of 500 Oe. Black lines represent the fits to the data using stretched exponential functions, which were used to extract $\tau$. 


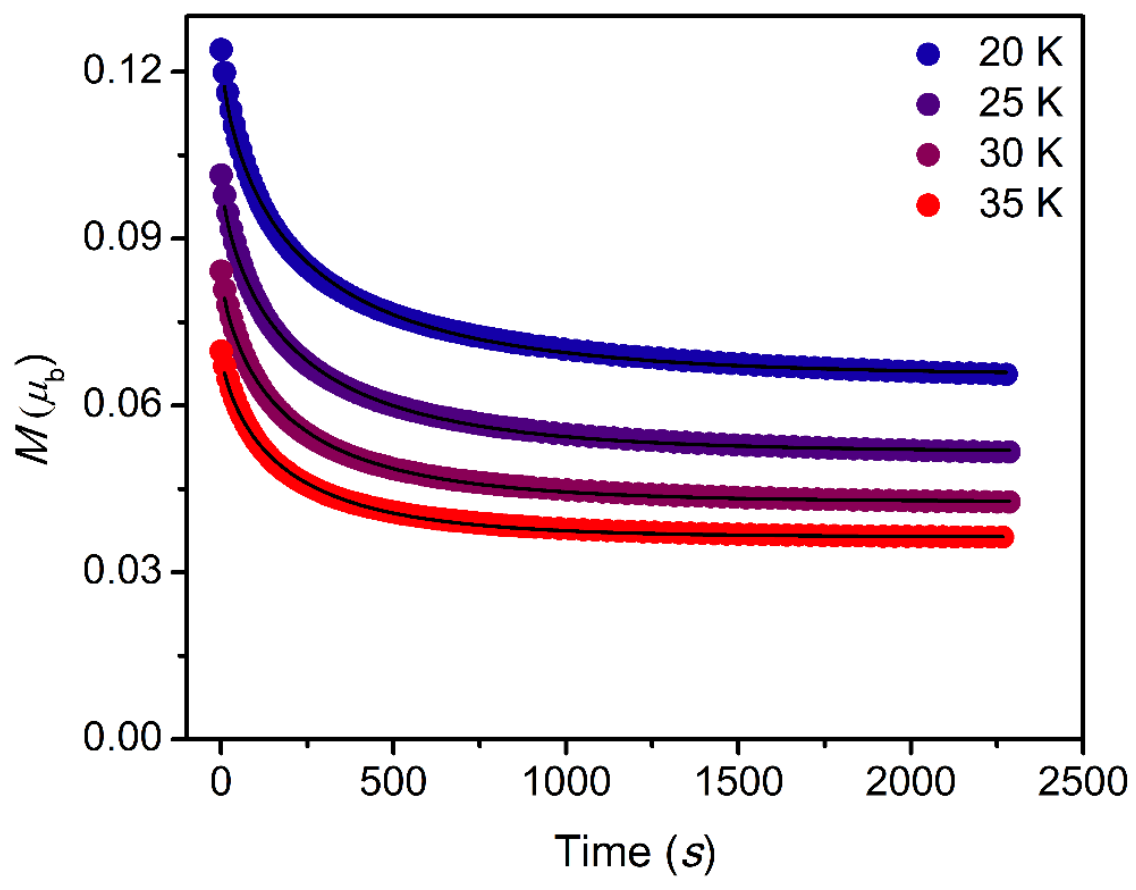

Figure S53. De magnetic relaxation data for 3 collected at 20, 25, 30, and $35 \mathrm{~K}$ and under an applied dc field of 500 Oe. Black lines represent the fits to the data using stretched exponential functions, which were used to extract $\tau$.

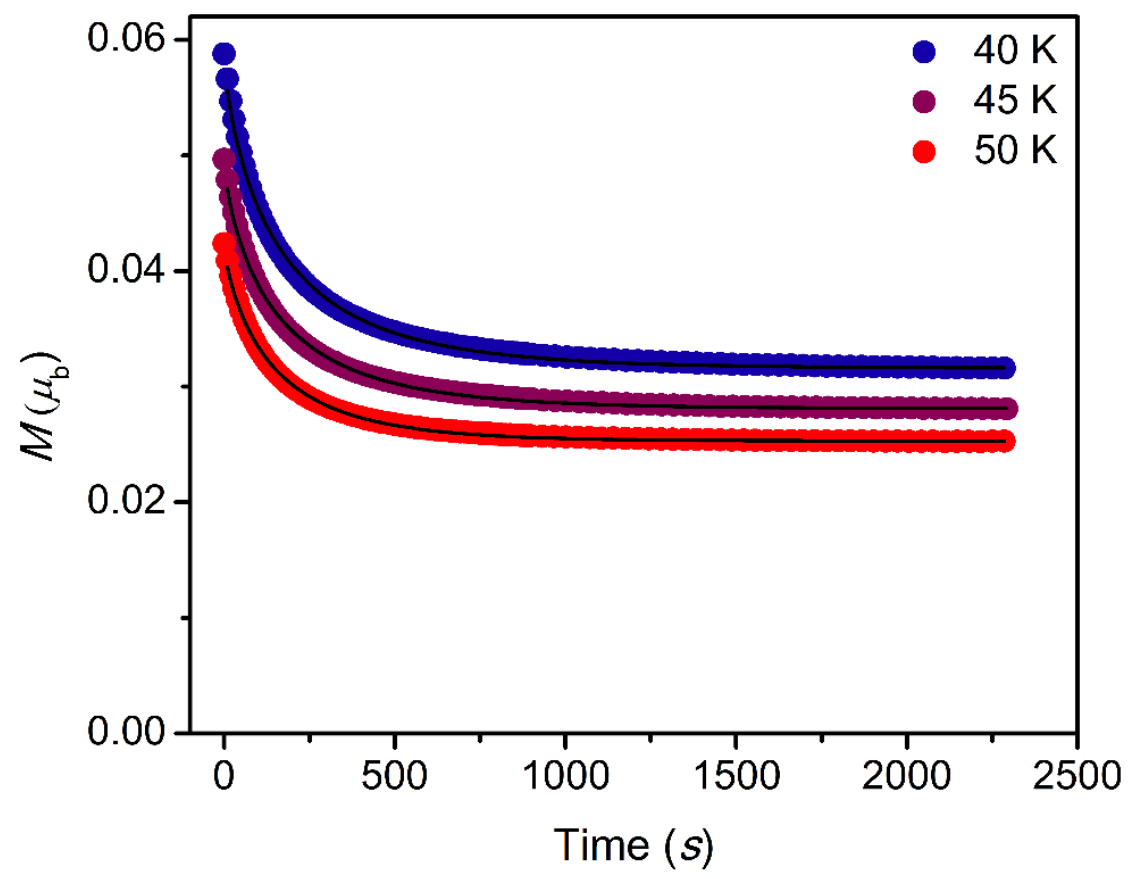

Figure S54. De magnetic relaxation data for 3 collected at 40, 45, and $50 \mathrm{~K}$ and under an applied dc field of 500 Oe. Black lines represent the fits to the data using stretched exponential functions, which were used to extract $\tau$. 
Table S8. Parameters used to fit dc magnetic relaxation data for a $28 \mathrm{mM}$ solution of $\mathbf{3}$ in toluene at zero applied magnetic field and magnetic relaxation times extracted from these fits.

\begin{tabular}{ccccc}
\hline $\boldsymbol{T}(\mathbf{K})$ & $\boldsymbol{M}_{\mathbf{0}}\left(\boldsymbol{\mu}_{\mathbf{b}}\right)$ & $\boldsymbol{M}_{\mathbf{1}}\left(\boldsymbol{\mu}_{\mathbf{b}}\right)$ & $\boldsymbol{n}$ & $\boldsymbol{\tau}\left(\mathbf{s}^{\mathrm{a}}\right.$ \\
\hline 2 & 0.71576 & $0.13849^{*}$ & 0.752 & 1652.206 \\
3 & 0.45533 & $0.06401^{*}$ & 0.806 & 967.568 \\
4 & 0.32806 & 0.04384 & 0.833 & 597.723 \\
5 & 0.27658 & 0.03951 & 0.793 & 405.136
\end{tabular}

${ }^{a}$ Values for $\tau$ are likely only accurate to three significant figures. The full value extracted from the fit is reported for reproducibility.

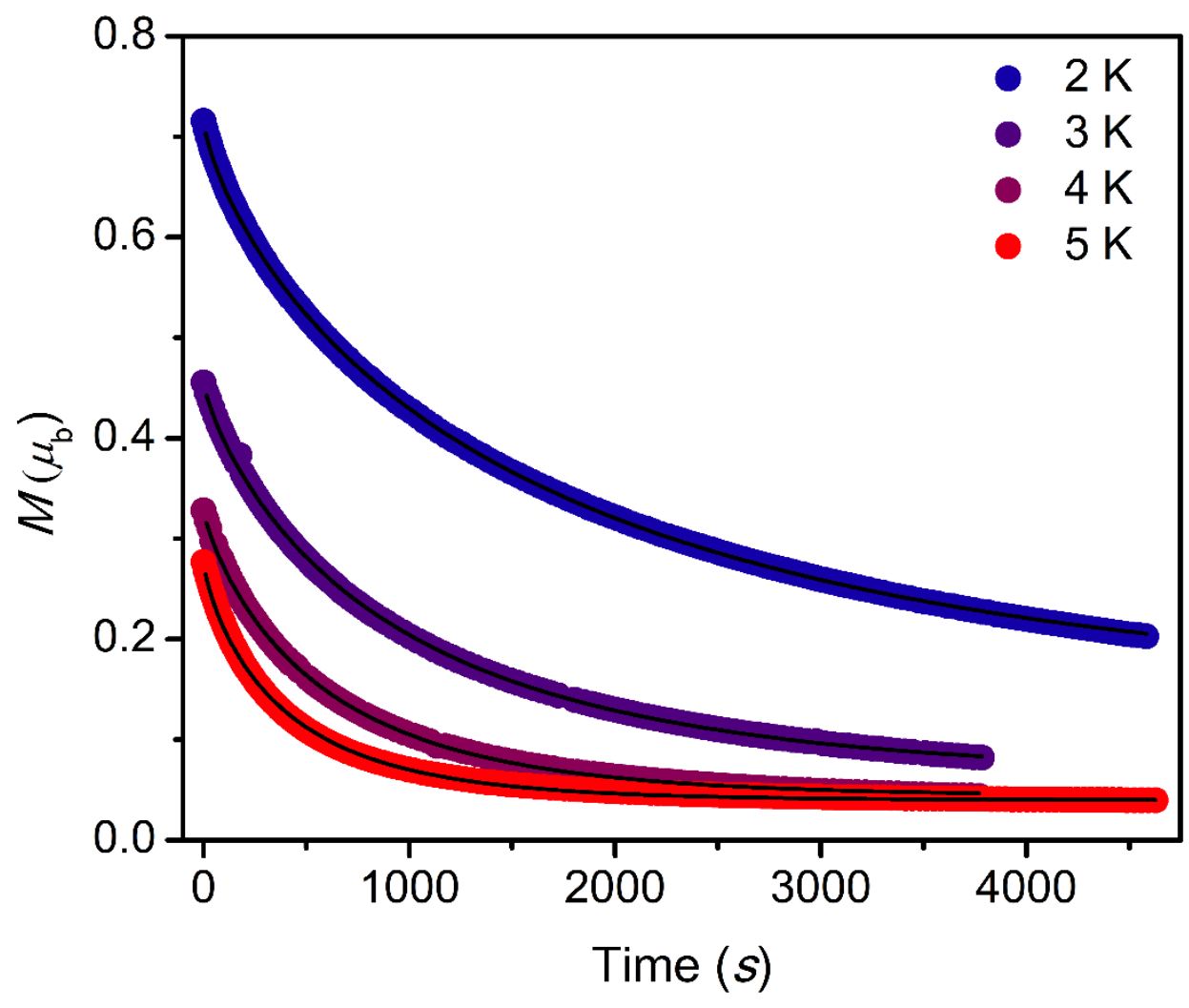

Figure S55. Dc magnetic relaxation data for a $28 \mathrm{mM}$ solution of $\mathbf{3}$ in toluene collected at 2, 3, 4, and $5 \mathrm{~K}$ and under an applied dc field of 0 Oe. Black lines represent the fits to the data using stretched exponential functions, which were used to extract $\tau$. 
Table S9. Parameters used to fit dc magnetic relaxation data for a $28 \mathrm{mM}$ solution of $\mathbf{3}$ in toluene at 500 Oe and magnetic relaxation times extracted from these fits.

\begin{tabular}{ccccc}
\hline $\boldsymbol{T}(\mathbf{K})$ & $\boldsymbol{M}_{\mathbf{0}}\left(\boldsymbol{\mu}_{\mathbf{b}}\right)$ & $\boldsymbol{M}_{\mathbf{1}}\left(\boldsymbol{\mu}_{\mathbf{b}}\right)$ & $\boldsymbol{n}$ & $\boldsymbol{\tau}(\mathbf{s})^{\mathrm{a}}$ \\
\hline 10 & 0.91364 & 0.16895 & 0.729 & 7499.979 \\
15 & 0.62748 & 0.07644 & 0.694 & 3311.353 \\
20 & 0.50794 & 0.05997 & 0.716 & 1872.765 \\
25 & 0.43559 & 0.04977 & 0.720 & 1333.256 \\
30 & 0.30633 & 0.04267 & 0.725 & 694.438 \\
35 & 0.26036 & 0.03719 & 0.745 & 681.890 \\
40 & 0.20410 & 0.03273 & 0.764 & 536.999 \\
45 & 0.15955 & 0.02902 & 0.790 & 433.028 \\
50 & 0.13100 & 0.02591 & 0.810 & 376.474 \\
\hline
\end{tabular}

${ }^{\text {a }}$ alues for $\tau$ are likely only accurate to three significant figures. The full value extracted from the fit is reported for reproducibility.

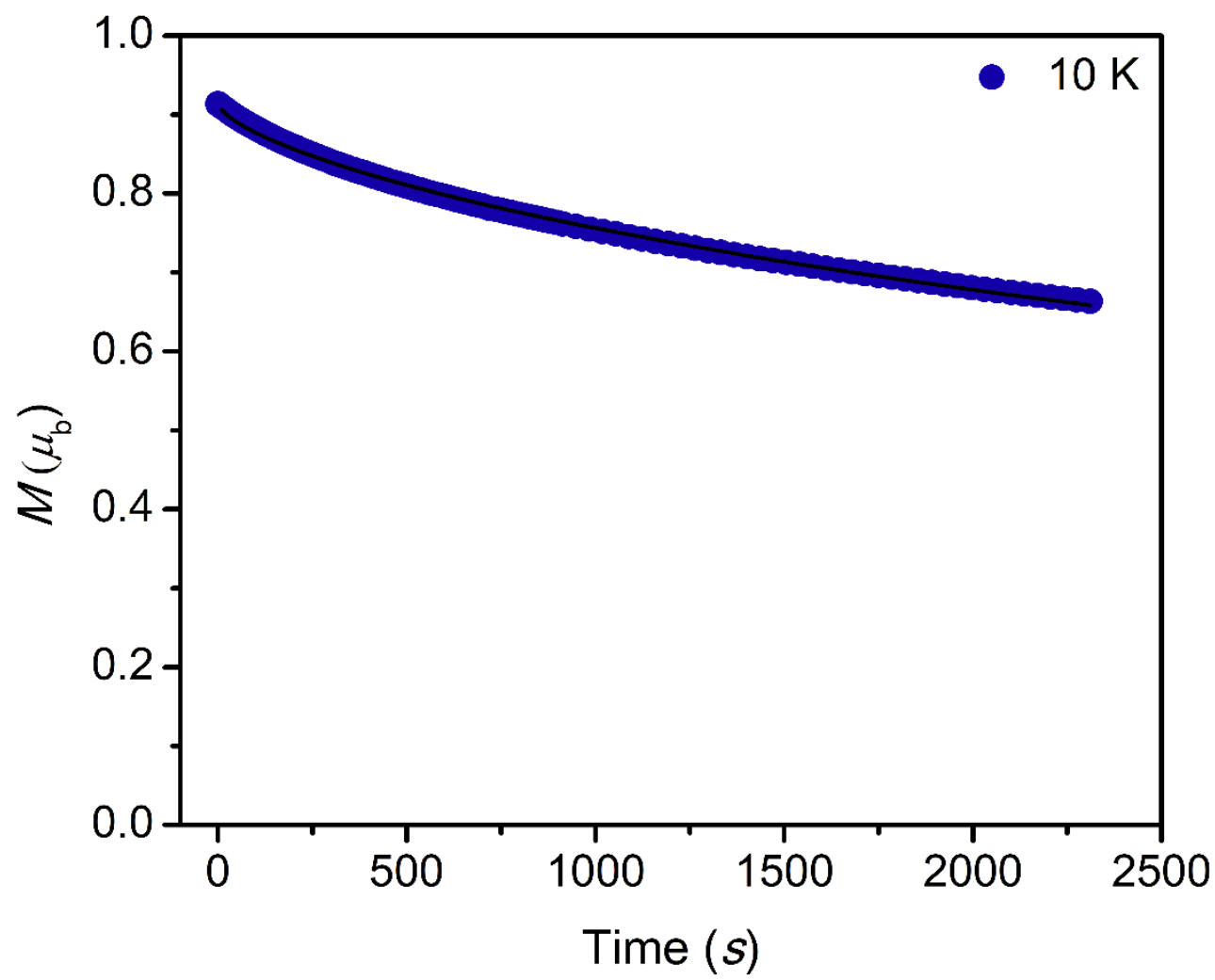

Figure S56. Dc magnetic relaxation data for a $28 \mathrm{mM}$ solution of $\mathbf{3}$ in toluene collected at $10 \mathrm{~K}$ and under an applied dc field of 500 Oe. Black lines represent the fits to the data using stretched exponential functions, which were used to extract $\tau$. 


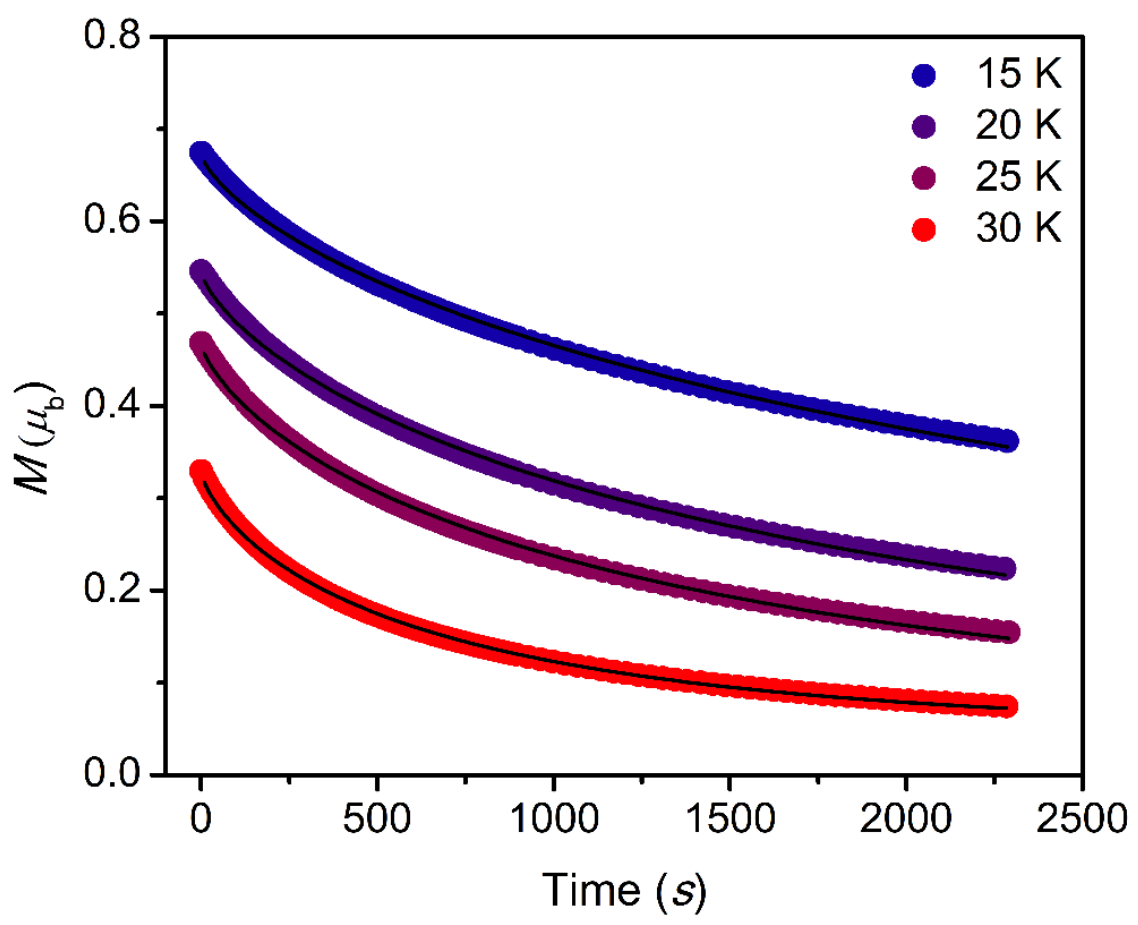

Figure S57. Dc magnetic relaxation data for a $28 \mathrm{mM}$ solution of $\mathbf{3}$ in toluene collected at 15, 20, 25 , and $30 \mathrm{~K}$ and under an applied dc field of $500 \mathrm{Oe}$. Black lines represent the fits to the data using stretched exponential functions, which were used to extract $\tau$.

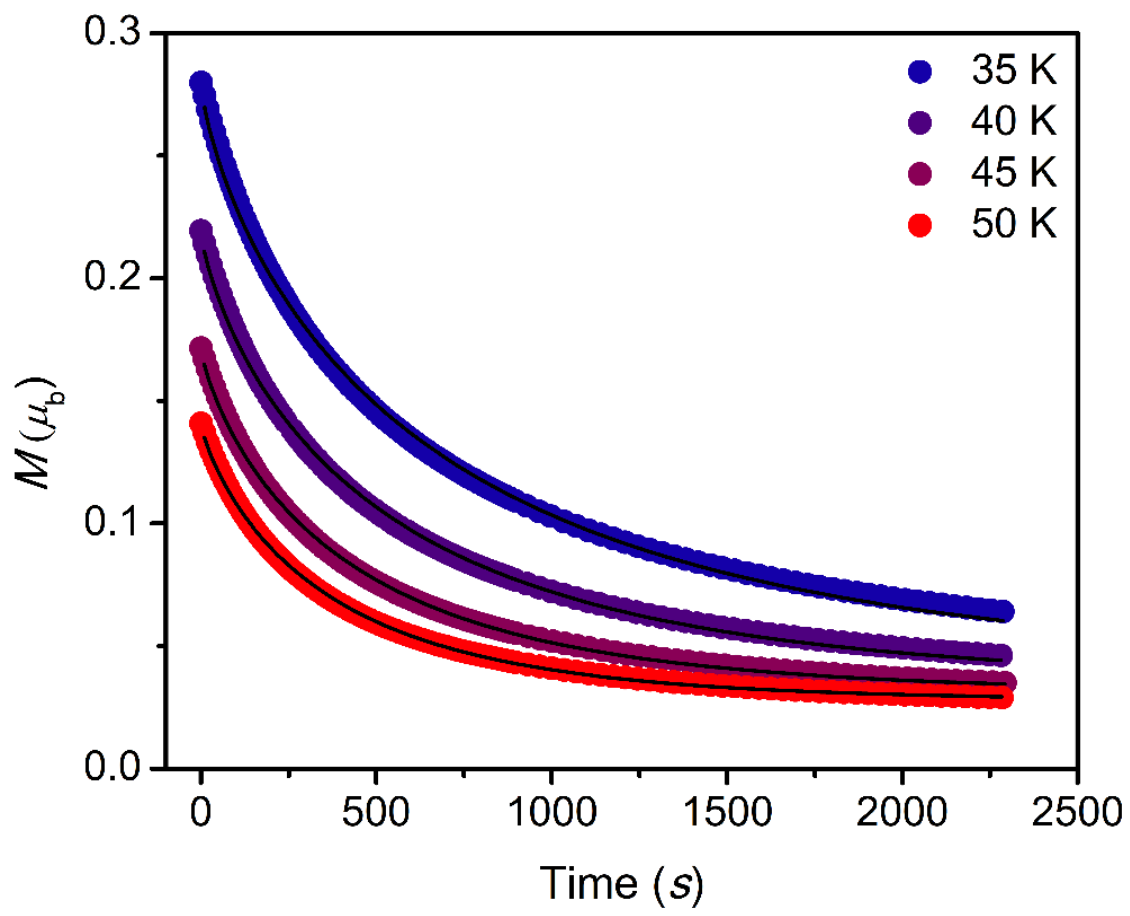

Figure S58. Dc magnetic relaxation data for a $28 \mathrm{mM}$ solution of $\mathbf{3}$ in toluene collected at 35, 40, 45 , and $50 \mathrm{~K}$ and under an applied dc field of 500 Oe. Black lines represent the fits to the data using stretched exponential functions, which were used to extract $\tau$. 
Analysis of Magnetic Relaxation Dynamics. The full range of magnetic relaxation times extracted from ac susceptibility and dc magnetic relaxation experiments of 1-3 showed a complex temperature dependence, indicative of multiple magnetic relaxation pathways. Plots of the log of magnetic relaxation time versus $1 / T$ showed an exponential dependence at intermediate temperatures and flattened at low $T$, suggesting the presence of Raman relaxation and/or quantum tunneling of the magnetization. Accordingly, the data for 1-4 were fit to the equation:

$$
\tau^{-1}=\tau_{0}^{-1} e^{-U_{e f f} / k_{b} T}+\tau_{\text {tunnel }}^{-1}+C T^{n}
$$

where $\tau_{\text {tunnel }}$ is the relaxation time for quantum tunneling of the magnetization and $C$ and $n$ are free variables that describe Raman relaxation. In the following tables, "n/a" represents data for which magnetic relaxation times could not be extracted (signals in the out-of-phase susceptibility were observed but the peak was beyond the detection limit of our magnetometer), while "not observed" corresponds to a lack of signals in ac magnetic susceptibility or dc magnetic relaxation measurements.

Table S10. Parameters used to fit the Arrhenius plots of $\mathbf{1 .}$

\begin{tabular}{|c|c|}
\hline & $\begin{array}{c}\text { Polycrystalline 1 } \\
H_{\mathrm{dc}}=0 \mathrm{Oe}\end{array}$ \\
\hline$U_{\text {eff }}\left(\mathrm{cm}^{-1}\right)$ & - \\
\hline$\tau_{0}(\mathrm{~s})$ & - \\
\hline$\tau_{\text {tunnel }}(\mathrm{s})$ & $5.72 \times 10^{-3}$ \\
\hline$C$ & 2.04 \\
\hline$n$ & 2.00 \\
\hline
\end{tabular}

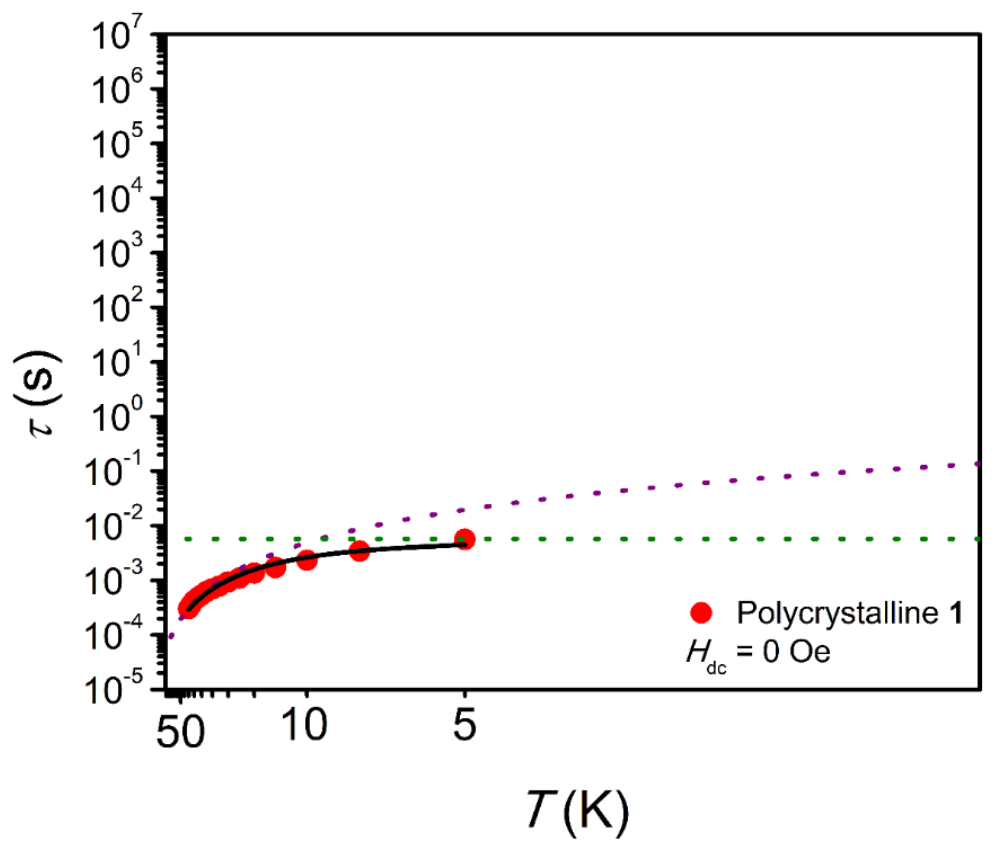

Figure S59. Plot of magnetic relaxation time versus temperature for 1. Green and purple lines represent fits to quantum tunneling and Raman relaxation processes, respectively. Black line represents the total fit to the data. 
Table S11. Parameters used to fit the Arrhenius plots of 2.

\begin{tabular}{|c|c|c|c|c|}
\hline $\begin{array}{c}\text { Fast } \\
\text { Process }\end{array}$ & $\begin{array}{c}\text { Polycrystalline 2 } \\
H_{\mathrm{dc}}=0 \mathrm{Oe}\end{array}$ & $\begin{array}{c}\text { Polycrystalline 2 } \\
H_{\mathrm{dc}}=500 \mathrm{Oe}\end{array}$ & $\begin{array}{c}19 \mathrm{mM} \text { Solution 2 } \\
H_{\mathrm{dc}}=0 \text { Oe }\end{array}$ & $\begin{array}{c}19 \mathrm{mM} \text { Solution 2 } \\
H_{\mathrm{dc}}=500 \text { Oe }\end{array}$ \\
\hline$U_{\mathrm{eff}}\left(\mathrm{cm}^{-1}\right)$ & - & - & $\mathrm{n} / \mathrm{a}$ & $\mathrm{n} / \mathrm{a}$ \\
\hline$\tau_{0}(\mathrm{~s})$ & - & - & $\mathrm{n} / \mathrm{a}$ & $\mathrm{n} / \mathrm{a}$ \\
\hline$\tau_{\text {tunnel }}(\mathrm{s})$ & $7.92 \times 10^{-1}$ & $7.92 \times 10^{-1}$ & $\mathrm{n} / \mathrm{a}$ & $\mathrm{n} / \mathrm{a}$ \\
\hline$C$ & - & - & $\mathrm{n} / \mathrm{a}$ & $\mathrm{n} / \mathrm{a}$ \\
\hline$n$ & - & - & $\mathrm{n} / \mathrm{a}$ & $\mathrm{n} / \mathrm{a}$ \\
\hline
\end{tabular}

\begin{tabular}{|c|c|c|c|c|}
\hline $\begin{array}{c}\text { Slow } \\
\text { Process }\end{array}$ & $\begin{array}{c}\text { Polycrystalline 2 } \\
H_{\mathrm{dc}}=0 \mathrm{Oe}\end{array}$ & $\begin{array}{c}\text { Polycrystalline 2 } \\
H_{\mathrm{dc}}=500 \mathrm{Oe}\end{array}$ & $\begin{array}{c}19 \mathrm{mM} \text { Solution 2 } \\
H_{\mathrm{dc}}=0 \mathrm{Oe}\end{array}$ & $\begin{array}{c}19 \mathrm{mM} \text { Solution 2 } \\
H_{\mathrm{dc}}=500 \mathrm{Oe}\end{array}$ \\
\hline$U_{\text {eff }}\left(\mathrm{cm}^{-1}\right)$ & 1205 & - & - & - \\
\hline$\tau_{0}(\mathrm{~s})$ & $2.46 \times 10^{-11}$ & - & - & - \\
\hline$\tau_{\text {tunnel }}(\mathrm{s})$ & $6.42 \times 10^{2}$ & $7.95 \times 10^{-2}$ & $2.54 \times 10^{2}$ & $2.99 \times 10^{6}$ \\
\hline$C$ & $9.69 \times 10^{-18}$ & $1.00 \times 10^{-13}$ & $9.69 \times 10^{-18}$ & $2.07 \times 10^{-10}$ \\
\hline$n$ & 8.69 & 6.31 & 8.69 & 4.22 \\
\hline
\end{tabular}

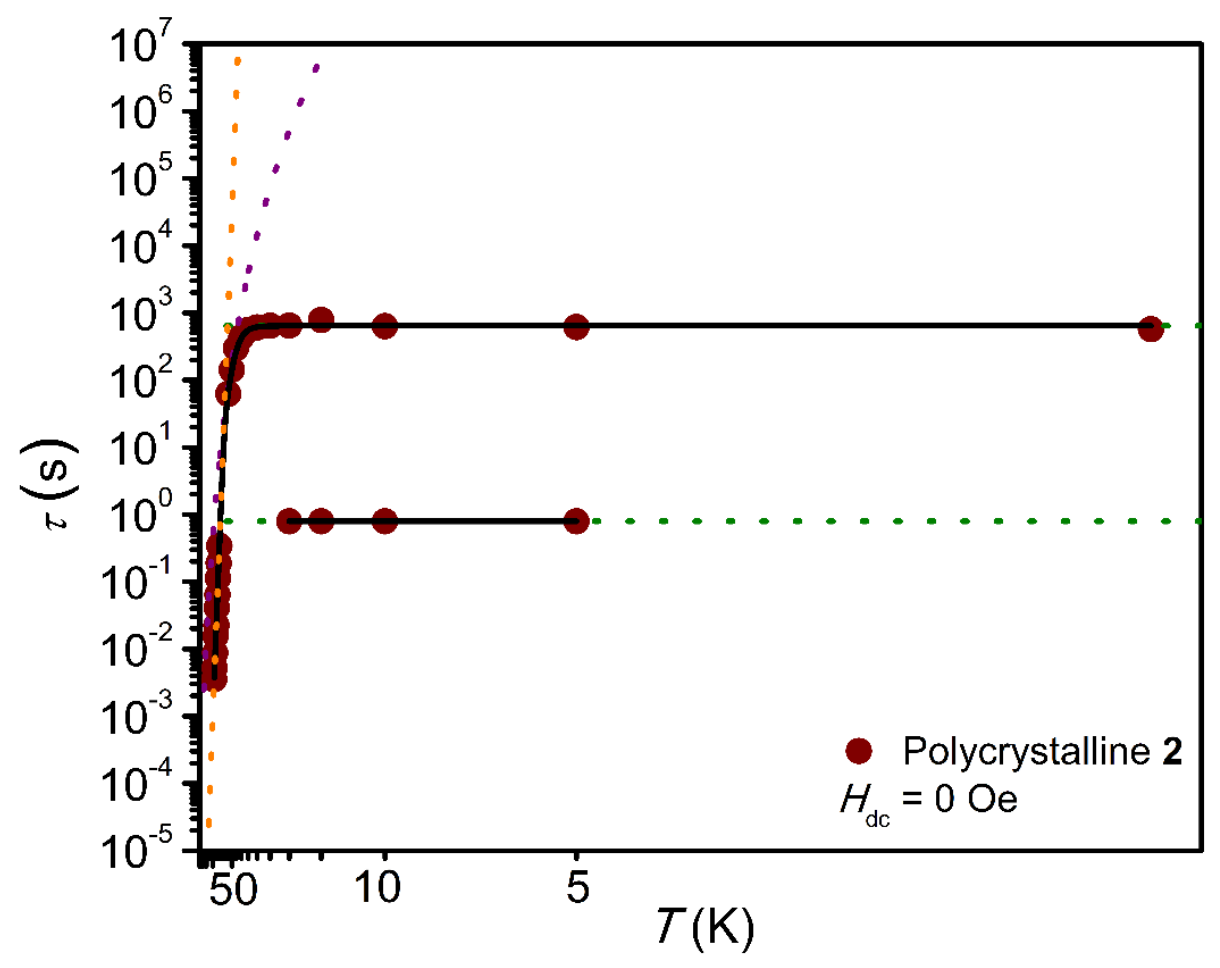

Figure S60. Plot of magnetic relaxation time versus temperature for a polycrystalline sample of 2 under zero applied dc field. Green, purple, and orange lines represent fits to quantum tunneling, Raman relaxation, and Orbach processes, respectively. Black line represents the total fit to the data. 


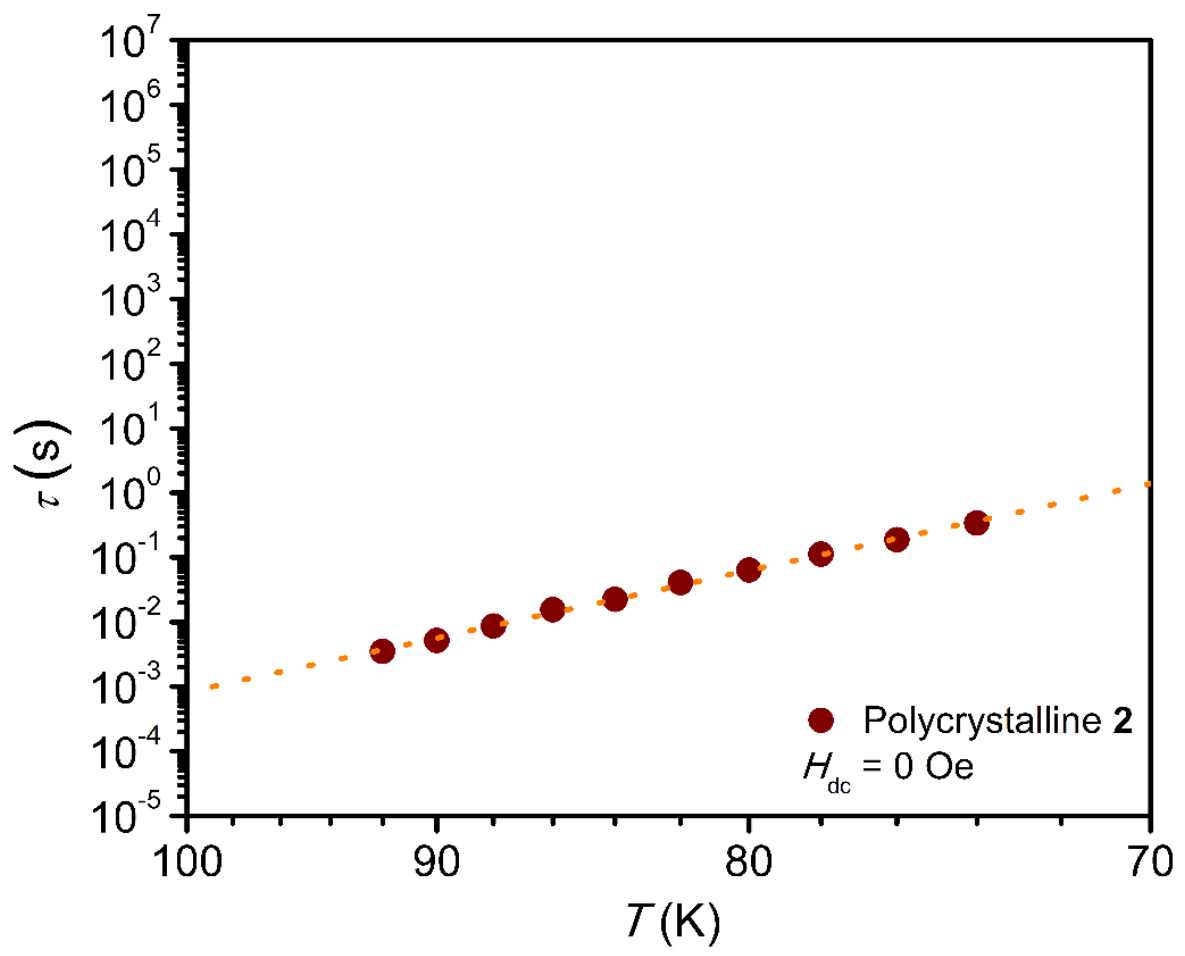

Figure S61. Plot of magnetic relaxation time versus temperature for a polycrystalline sample of 2 under zero applied dc field from 70 to $100 \mathrm{~K}$. Orange line represent fits to an Orbach processes.

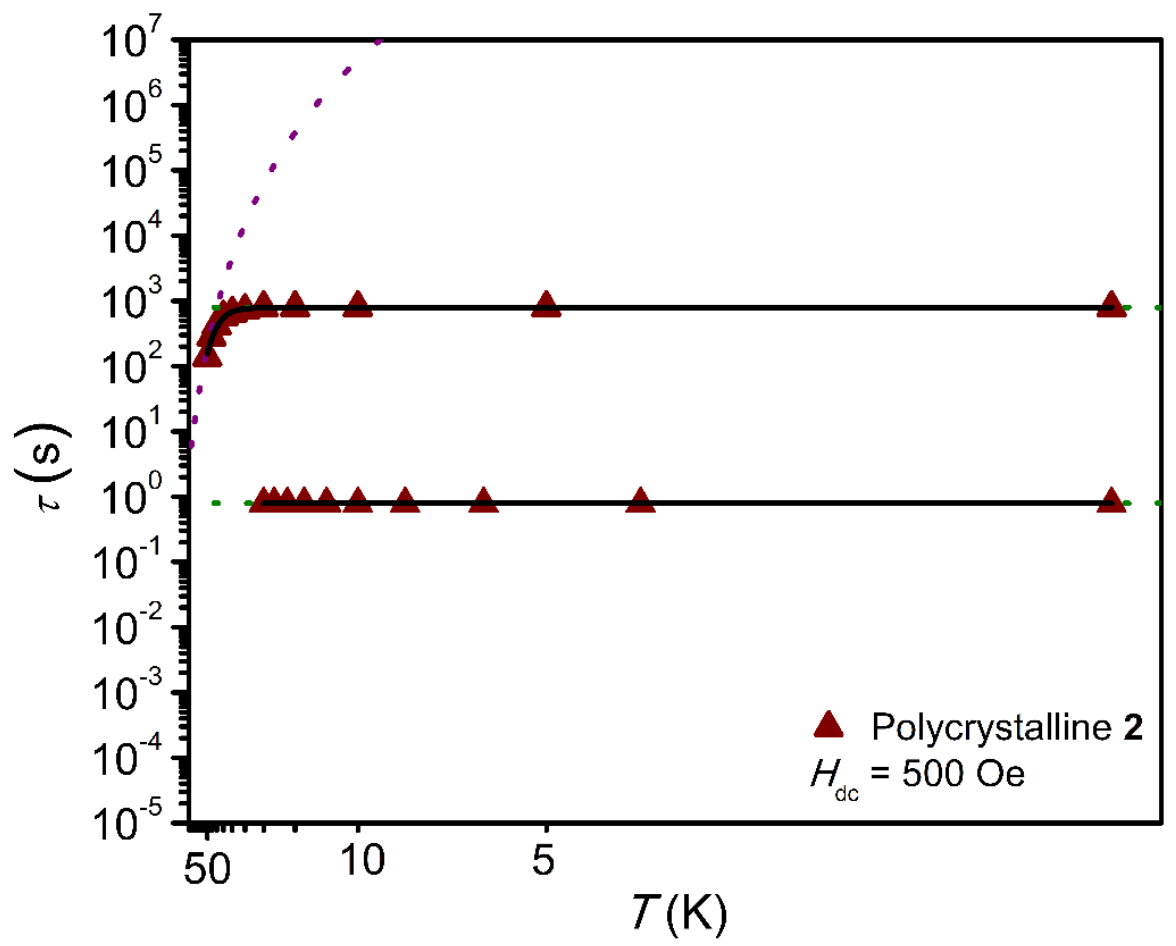

Figure S62. Plot of magnetic relaxation time versus temperature for a polycrystalline sample of 2 under an applied dc field of 500 Oe. Green and purple lines represent fits to quantum tunneling and Raman relaxation processes, respectively. Black line represents the total fit to the data 


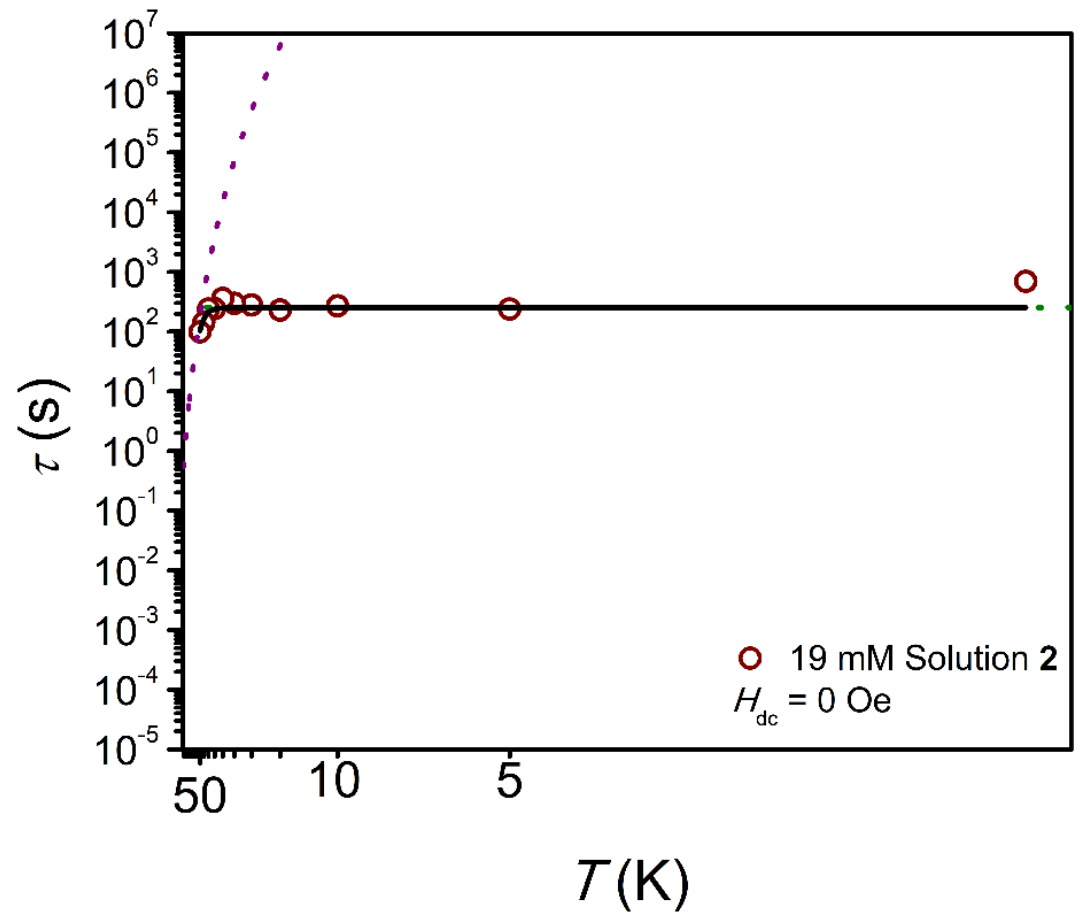

Figure S63. Plot of magnetic relaxation time versus temperature for a $19 \mathrm{mM}$ solution of 2 in toluene under zero applied dc field. Green and purple lines represent fits to quantum tunneling and Raman relaxation processes, respectively. Black line represents the total fit to the data.

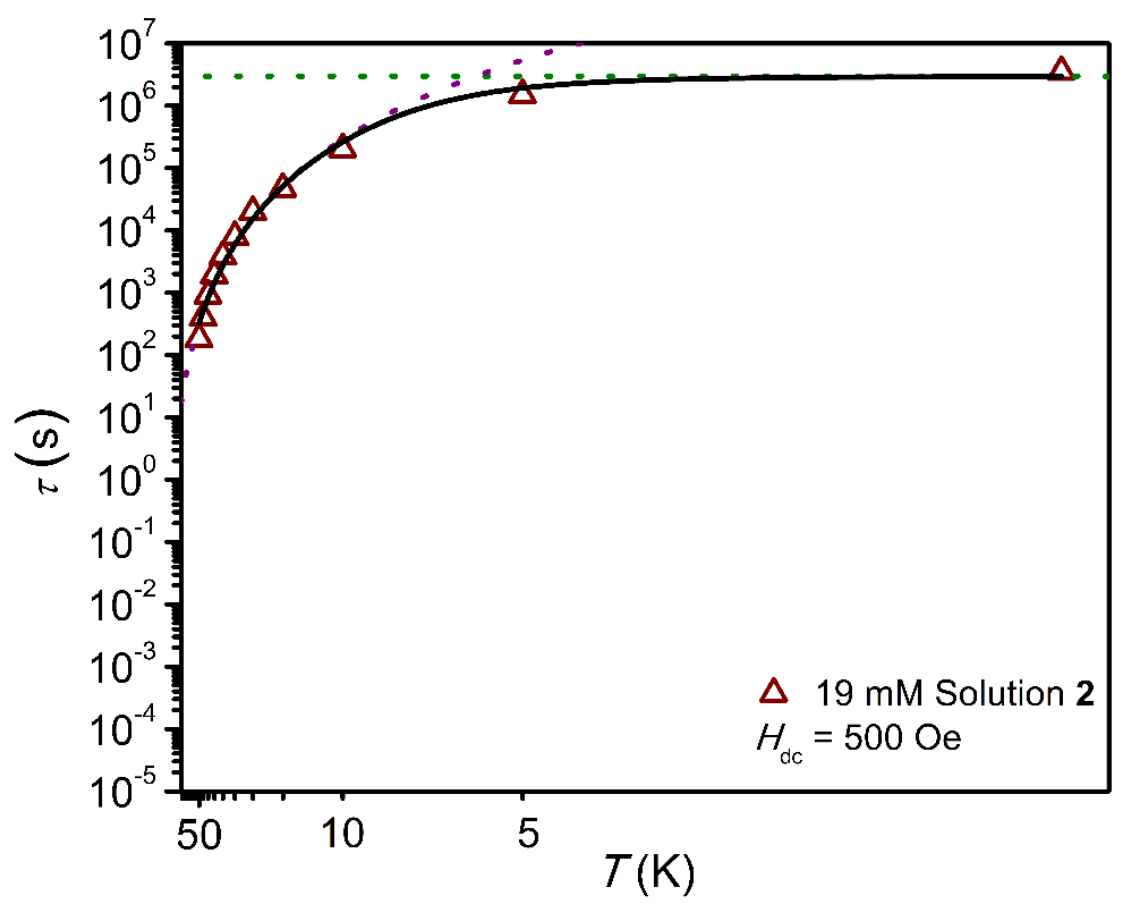

Figure S64. Plot of magnetic relaxation time versus temperature for a $19 \mathrm{mM}$ solution of 2 in toluene under an applied dc field of $500 \mathrm{Oe}$. Green and purple lines represent fits to quantum tunneling and Raman relaxation processes, respectively. Black line represents the total fit to the data. 


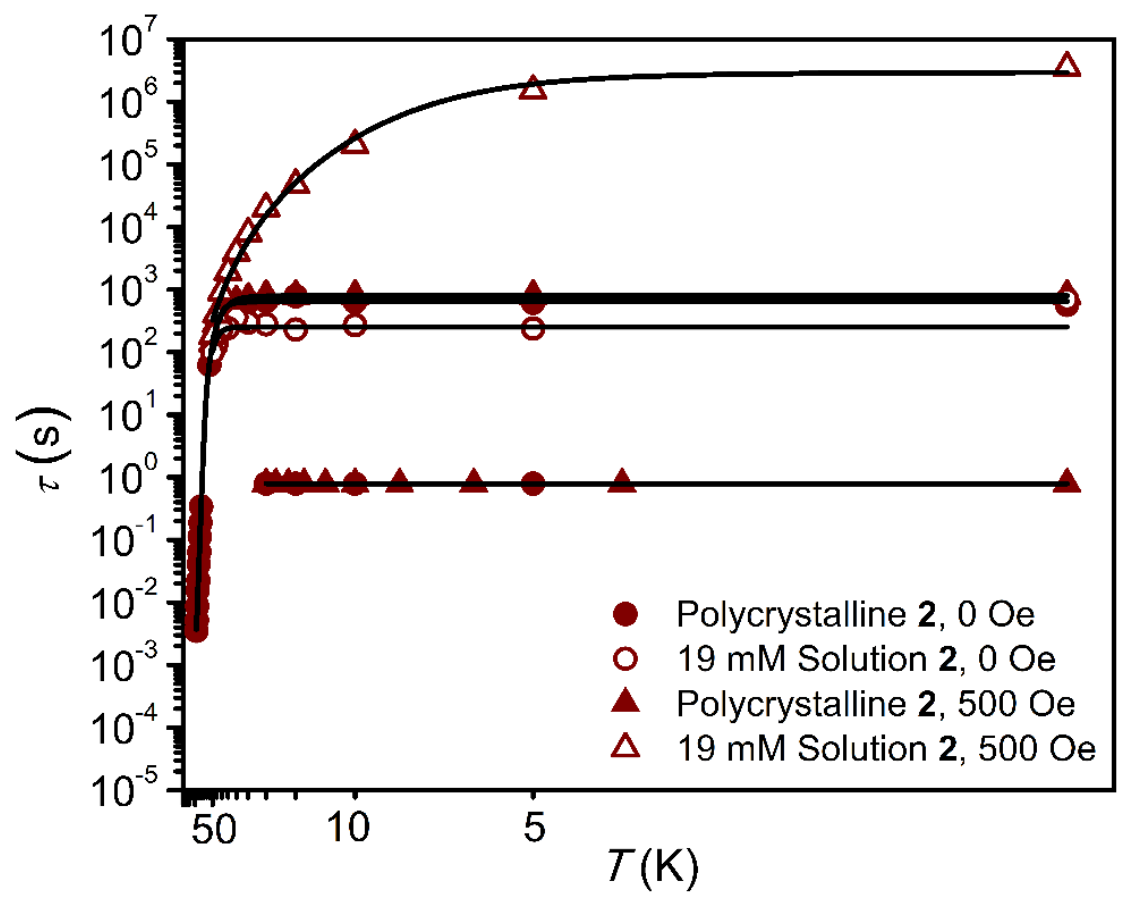

Figure S65. Plot of magnetic relaxation time versus temperature for a polycrystalline sample and a $19 \mathrm{mM}$ solution of $\mathbf{2}$ under zero applied dc field and an applied dc field of 500 Oe. Black lines represent overall fits to the data. The fast tunneling process disappears upon dilution, suggesting that it may be due to dipolar interactions. The slow process overlays at high temperatures for both zero and applied fields, as well as in polycrystalline and solution samples, suggesting that it is molecular in origin. The rate of tunneling in this slow process decreases upon application on an external field, as expected. 
Table S12. Parameters used to fit the Arrhenius plots of $\mathbf{3}$.

\begin{tabular}{|c|c|c|c|c|}
\hline $\begin{array}{c}\text { Fast } \\
\text { Process }\end{array}$ & $\begin{array}{c}\text { Polycrystalline 3 } \\
H_{\mathrm{dc}}=0 \mathrm{Oe}\end{array}$ & $\begin{array}{c}\text { Polycrystalline 3 } \\
H_{\mathrm{dc}}=500 \mathrm{Oe}\end{array}$ & $\begin{array}{c}28 \mathrm{mM} \text { Solution 3 } \\
H_{\mathrm{dc}}=0 \text { Oe }\end{array}$ & $\begin{array}{c}28 \mathrm{mM} \text { Solution 3 } \\
H_{\mathrm{dc}}=500 \text { Oe }\end{array}$ \\
\hline$U_{\mathrm{eff}}\left(\mathrm{cm}^{-1}\right)$ & - & 37.4 & 37.4 & 37.4 \\
\hline$\tau_{0}(\mathrm{~s})$ & - & $9.57 \times 10^{-6}$ & $9.57 \times 10^{-6}$ & $1.30 \times 10^{-5}$ \\
\hline$\tau_{\text {tunnel }}(\mathrm{s})$ & $1.54 \times 10^{-4}$ & - & - & - \\
& $2.00 \times 10^{-1}$ & & & $1.43 \times 10^{-2}$ \\
\hline$C$ & - & $5.63 \times 10^{-3}$ & $9.33 \times 10^{-1}$ & 3.65 \\
\hline$n$ & - & 4.34 & 2.00 & \\
\hline
\end{tabular}

\begin{tabular}{|c|c|c|c|c|}
\hline $\begin{array}{c}\text { Slow } \\
\text { Process }\end{array}$ & $\begin{array}{c}\text { Polycrystalline 3 } \\
H_{\mathrm{dc}}=0 \mathrm{Oe}\end{array}$ & $\begin{array}{c}\text { Polycrystalline 3 } \\
H_{\mathrm{dc}}=500 \mathrm{Oe}\end{array}$ & $\begin{array}{c}28 \mathrm{mM} \text { Solution } \mathbf{3} \\
H_{\mathrm{dc}}=0 \mathrm{Oe}\end{array}$ & $\begin{array}{c}28 \mathrm{mM} \text { Solution 3 } \\
H_{\mathrm{dc}}=500 \mathrm{Oe}\end{array}$ \\
\hline$U_{\text {eff }}\left(\mathrm{cm}^{-1}\right)$ & Not observed & - & - & - \\
\hline$\tau_{0}(\mathrm{~s})$ & Not observed & - & - & - \\
\hline$\tau_{\text {tunnel }}(\mathrm{s})$ & Not observed & $2.24 \times 10^{2}$ & $1.65 \times 10^{3}$ & $1.49 \times 10^{4}$ \\
\hline$C$ & Not observed & $2.87 \times 10^{-8}$ & $1.29 \times 10^{-5}$ & $4.94 \times 10^{-7}$ \\
\hline$n$ & Not observed & 2.89 & 3.11 & 2.22 \\
\hline
\end{tabular}

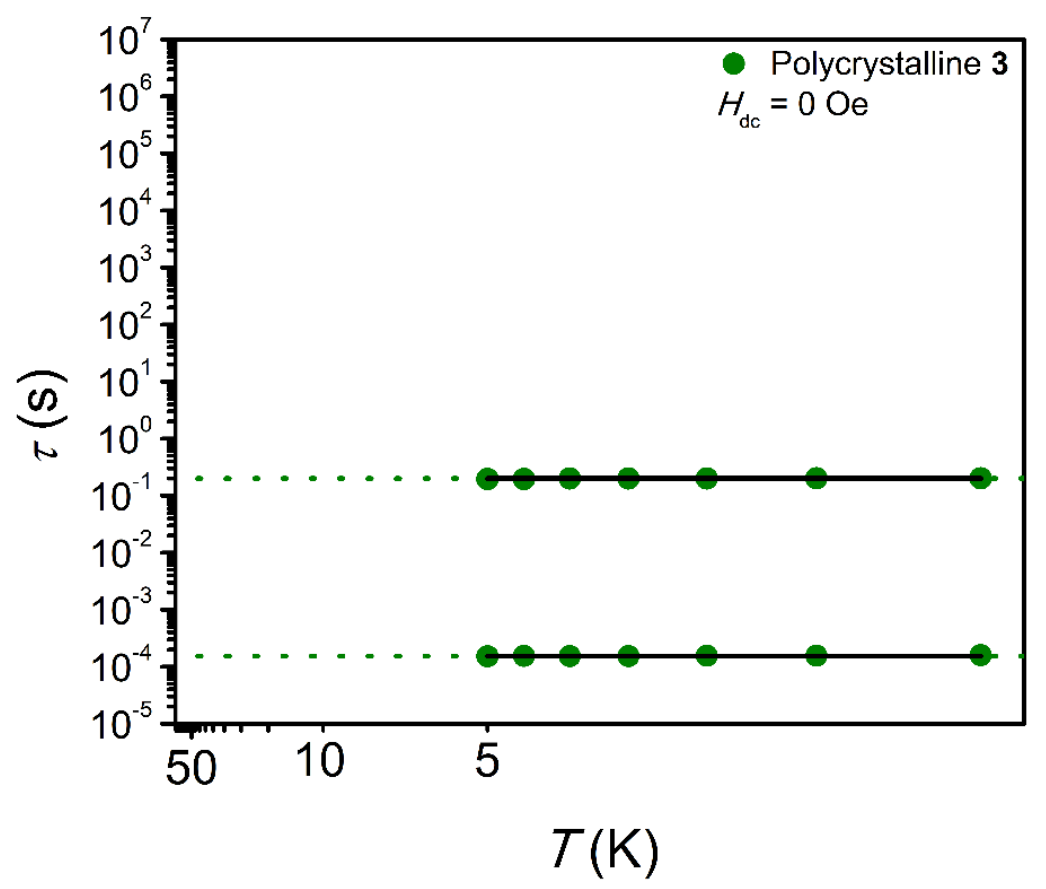

Figure S66. Plot of magnetic relaxation time versus temperature for a polycrystalline sample of $\mathbf{3}$ under zero applied dc field. Green lines represent fits to quantum tunneling processes. Black line represents the total fit to the data. Two relaxation processes were observed in the ac susceptibility measurements. 


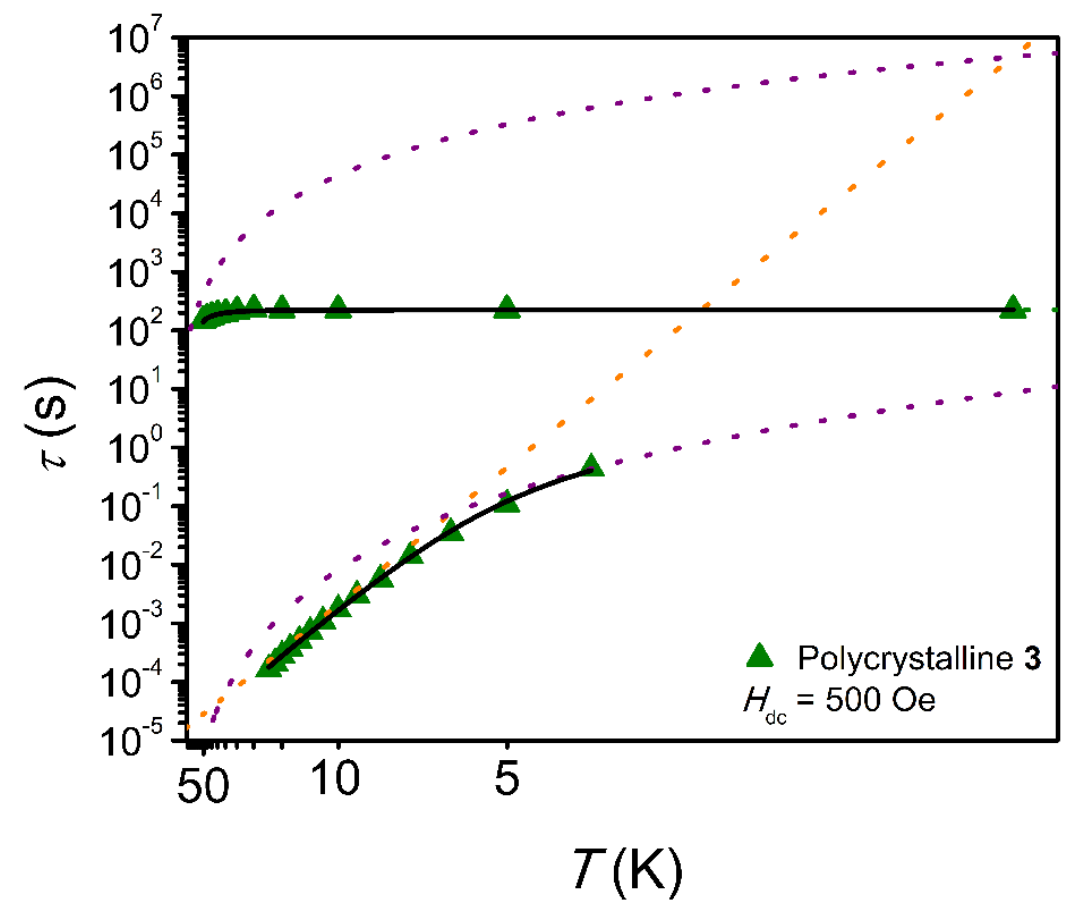

Figure S67. Plot of magnetic relaxation time versus temperature for a polycrystalline sample of $\mathbf{3}$ under an applied dc field of 500 Oe. Green, purple, and orange lines represent fits to quantum tunneling, Raman relaxation, and Orbach processes, respectively. Black line represents the total fit to the data.

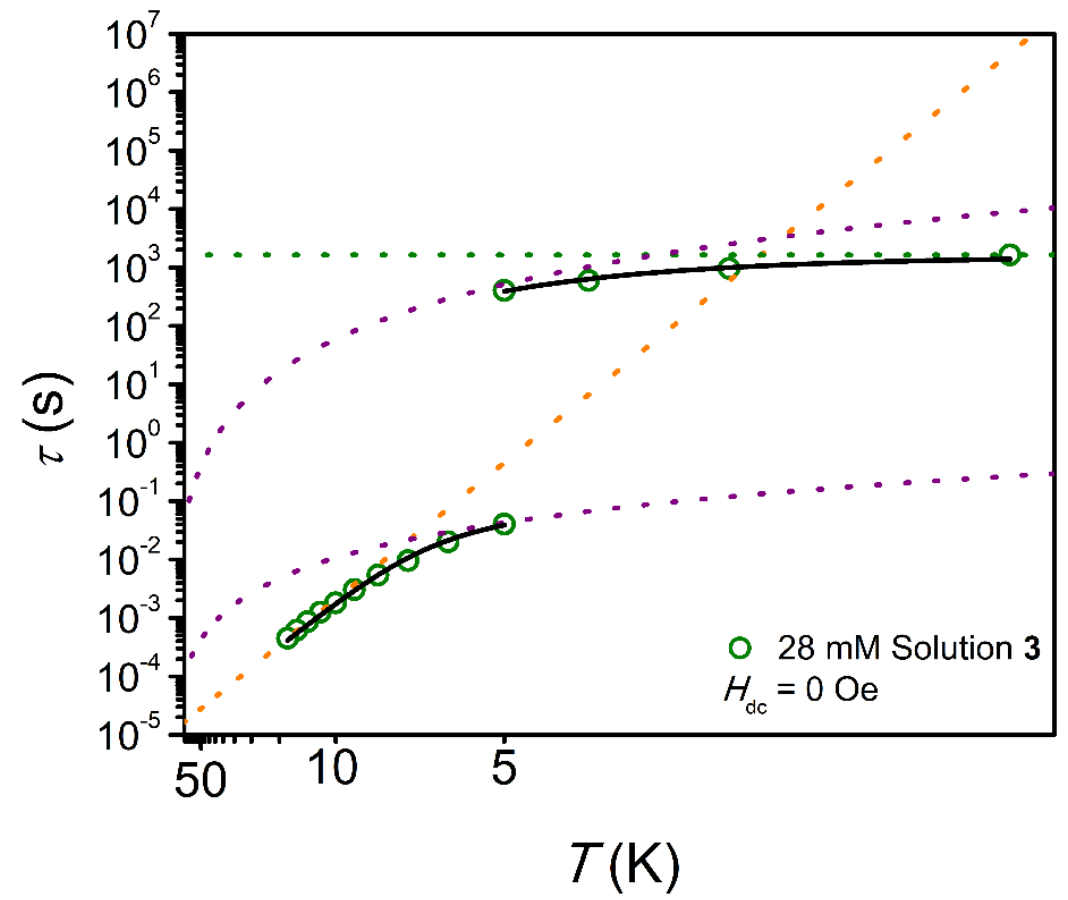

Figure S68. Plot of magnetic relaxation time versus temperature for a $28 \mathrm{mM}$ solution of $\mathbf{3}$ in toluene under zero applied dc field. Green, purple, and orange lines represent fits to quantum tunneling, Raman relaxation, and Orbach processes, respectively. Black line represents the total fit to the data. 


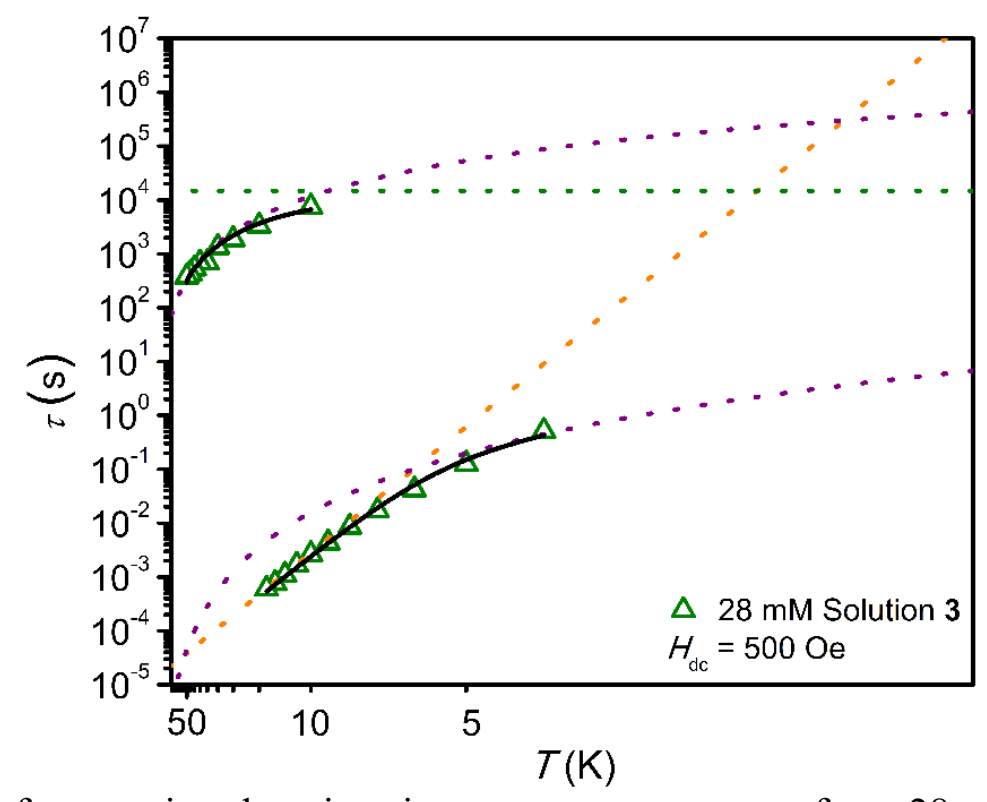

Figure S69. Plot of magnetic relaxation time versus temperature for a $28 \mathrm{mM}$ solution of $\mathbf{3}$ in toluene under an applied dc field of 500 Oe. Green, purple, and orange lines represent fits to quantum tunneling, Raman relaxation, and Orbach processes, respectively. Black line represents the total fit to the data.

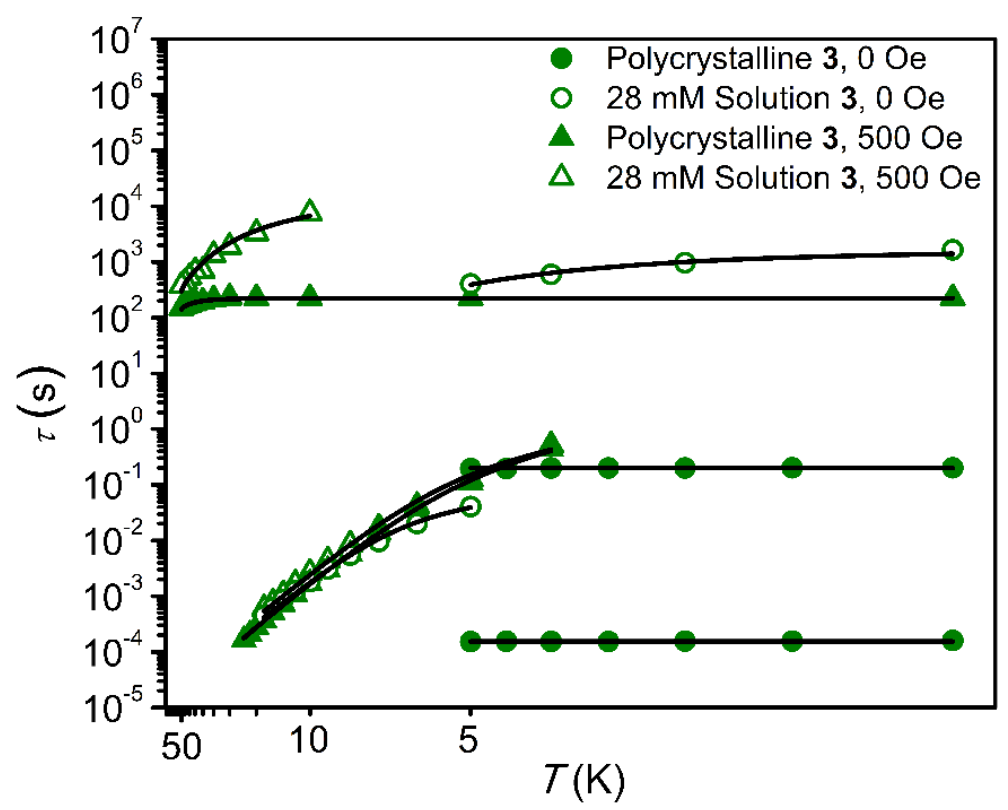

Figure S70. Plot of magnetic relaxation time versus temperature for a polycrystalline sample and a $28 \mathrm{mM}$ solution of $\mathbf{3}$ under zero applied field and an applied dc field of 500 Oe. Black lines represent overall fits to the data. The slow process overlays at high temperatures for polycrystalline and solution samples, suggesting that it is molecular in origin. The fast process with a linear dependence on temperature overlays for both zero and applied fields, as well as in polycrystalline and solution samples, suggesting that it is molecular in origin. The fast tunneling processes observed for polycrystalline $\mathbf{3}$ under zero applied field disappear in solution, suggesting that these relaxation processes may be dipolar in origin. 
Magnetic Hysteresis Measurements. Magnetic hysteresis measurements were collected on 1-3 at a sweep rate of 10.3(7) $\mathrm{mT} / \mathrm{s}$ for 1 and at sweep rates of 14.7(1) $\mathrm{mT} / \mathrm{s}$ for $H>2 \mathrm{~T}$ and 3.9(2) $\mathrm{mT} / \mathrm{s}$ for $H<2 \mathrm{~T}$ for 2 and 3. Magnetic hysteresis measurements were also conducted on a $19 \mathrm{mM}$ solution of $\mathbf{2}$ in toluene and on a $28 \mathrm{mM}$ solution of $\mathbf{3}$ in toluene in order to confirm that the magnetic behavior was molecular in origin. Coercivity decreased slightly in $\mathbf{2}$ which may be due to a change in molecular symmetry or metal-ligand vibrational modes upon dissolution. A decrease in coercivity was also reported for frozen solution measurements of $\left[\mathrm{Dy}\left(\mathrm{Cp}^{\mathrm{ttt}}\right)_{2}\right]\left[\mathrm{B}\left(\mathrm{C}_{6} \mathrm{~F}_{5}\right)_{4}\right]$ $\left(\mathrm{Cp}^{\mathrm{ttt}}=1,2,4\right.$-tri(tert-butyl)cyclopentadienyl). While hysteresis loops are closed at zero field for polycrystalline samples of $\mathbf{3}$, they open up to $10 \mathrm{~K}$ for frozen solution measurements. This is likely due to a decrease in dipolar interactions upon dilution. Such dipolar interactions often facilitate fast magnetic relaxation and thus removing them allows the molecular slow magnetic relaxation to be observed.

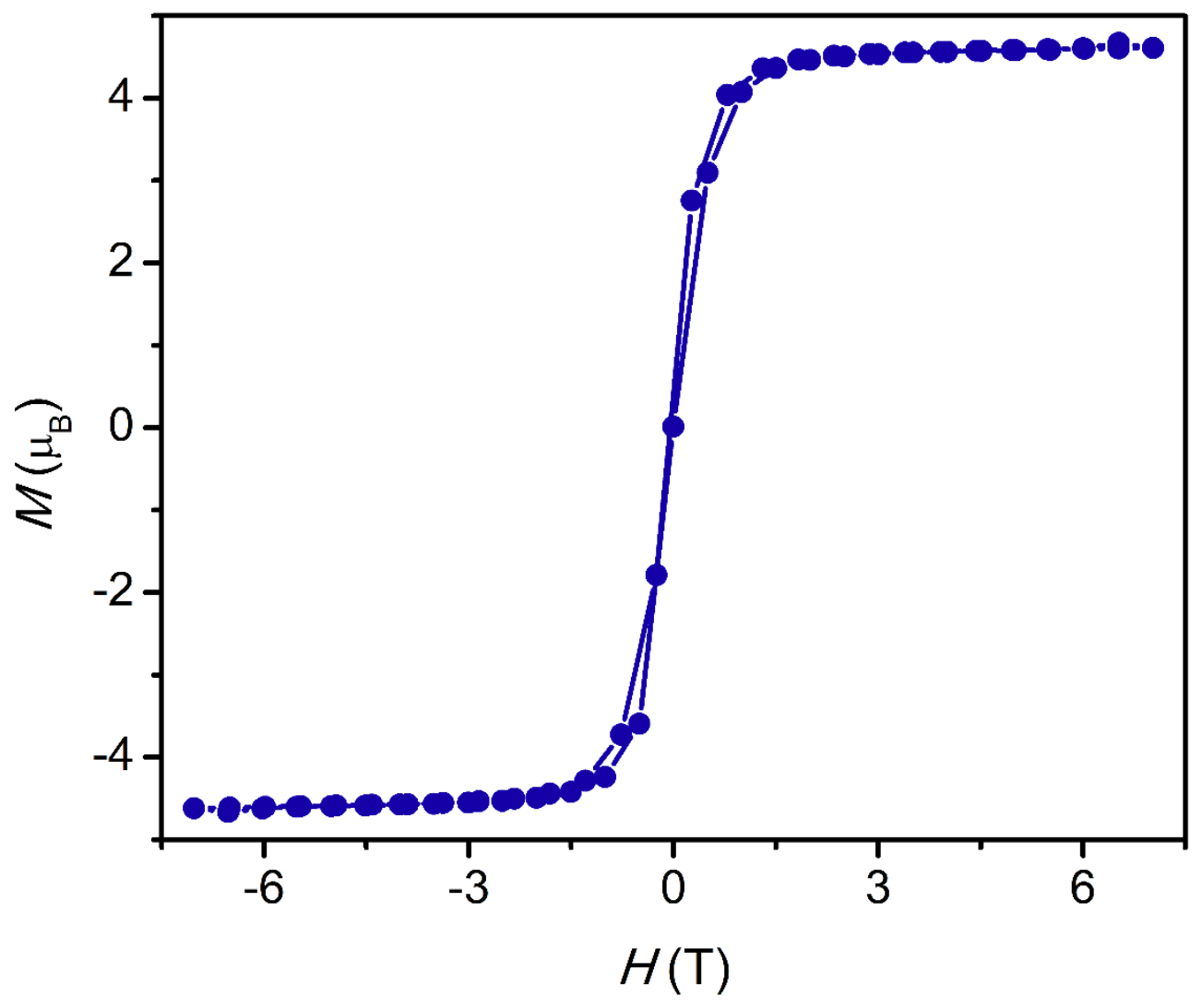

Figure S71. Magnetic hysteresis measurements of 1 at $2 \mathrm{~K}$ at a sweep rate of 10.3(7) mT/s. 


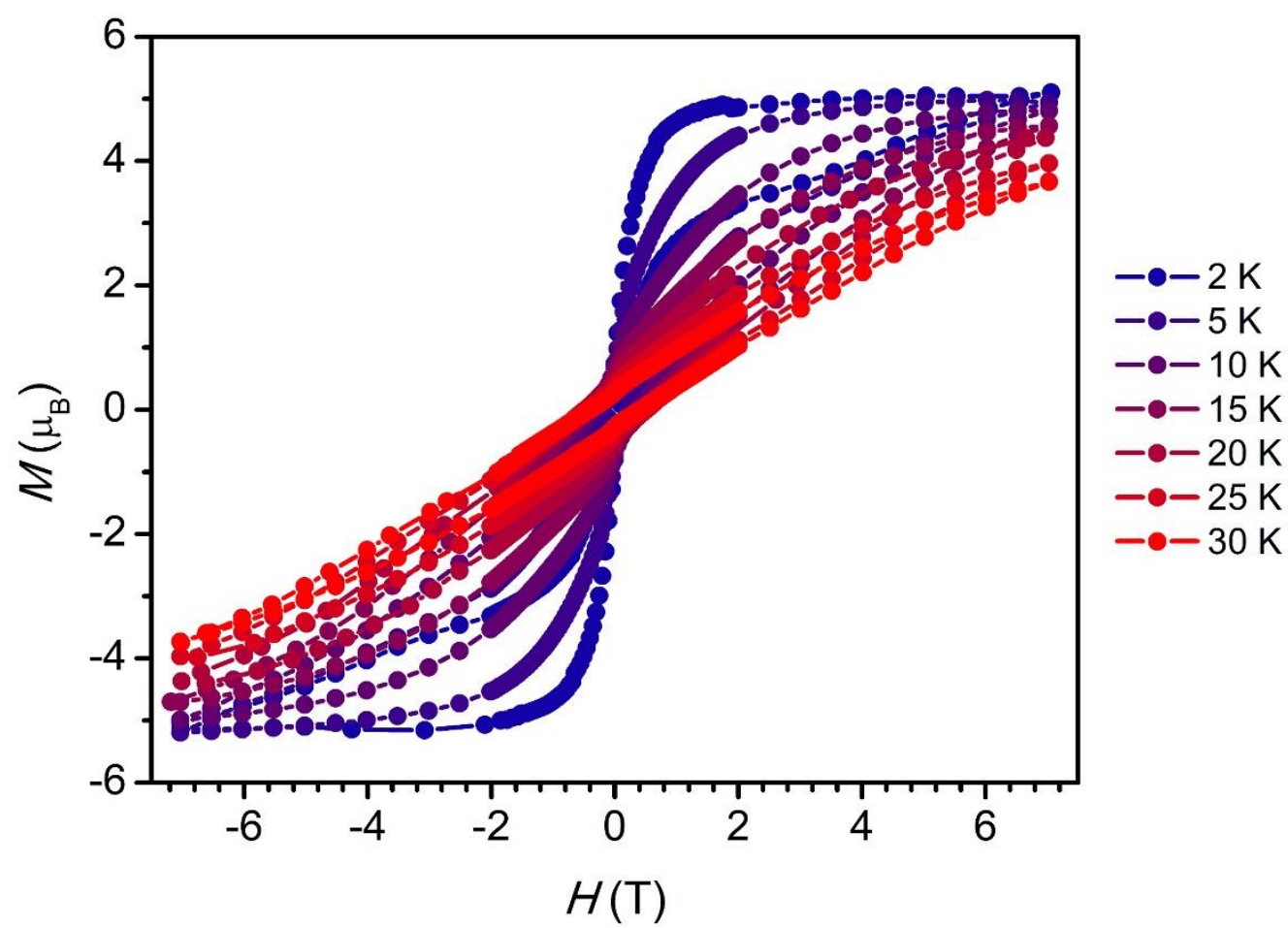

Figure S72. Magnetic hysteresis measurements of polycrystalline 2 from 2 to $30 \mathrm{~K}$ at a sweep rate of 14.7(1) mT/s for $H>2 \mathrm{~T}$ and 3.9(2) mT/s for $H<2 \mathrm{~T}$.

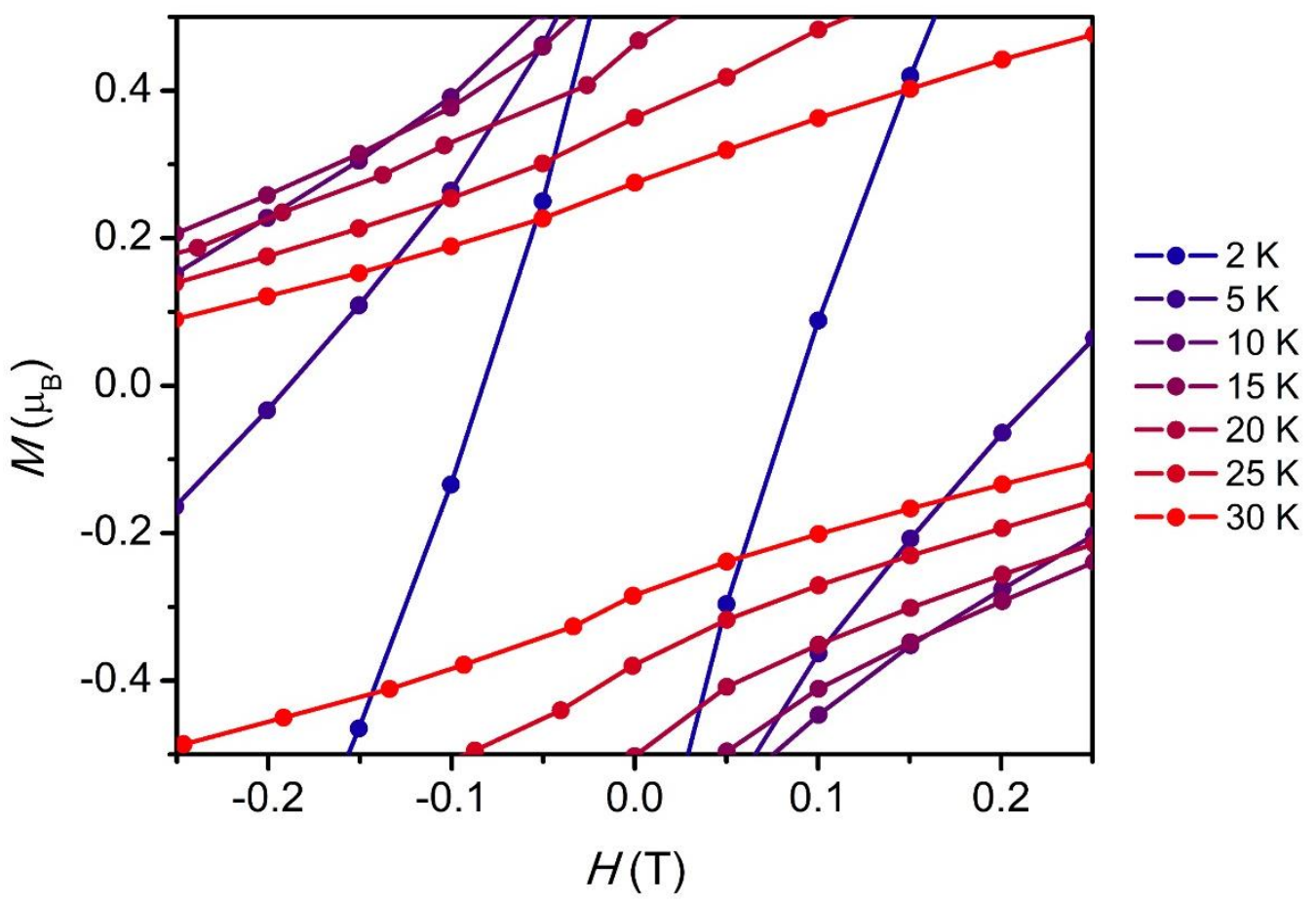

Figure S73. Magnetic hysteresis measurements of polycrystalline 2 from 2 to $30 \mathrm{~K}$ at a sweep rate of 14.7(1) mT/s for $H>2 \mathrm{~T}$ and 3.9(2) $\mathrm{mT} / \mathrm{s}$ for $H<2 \mathrm{~T}$. 


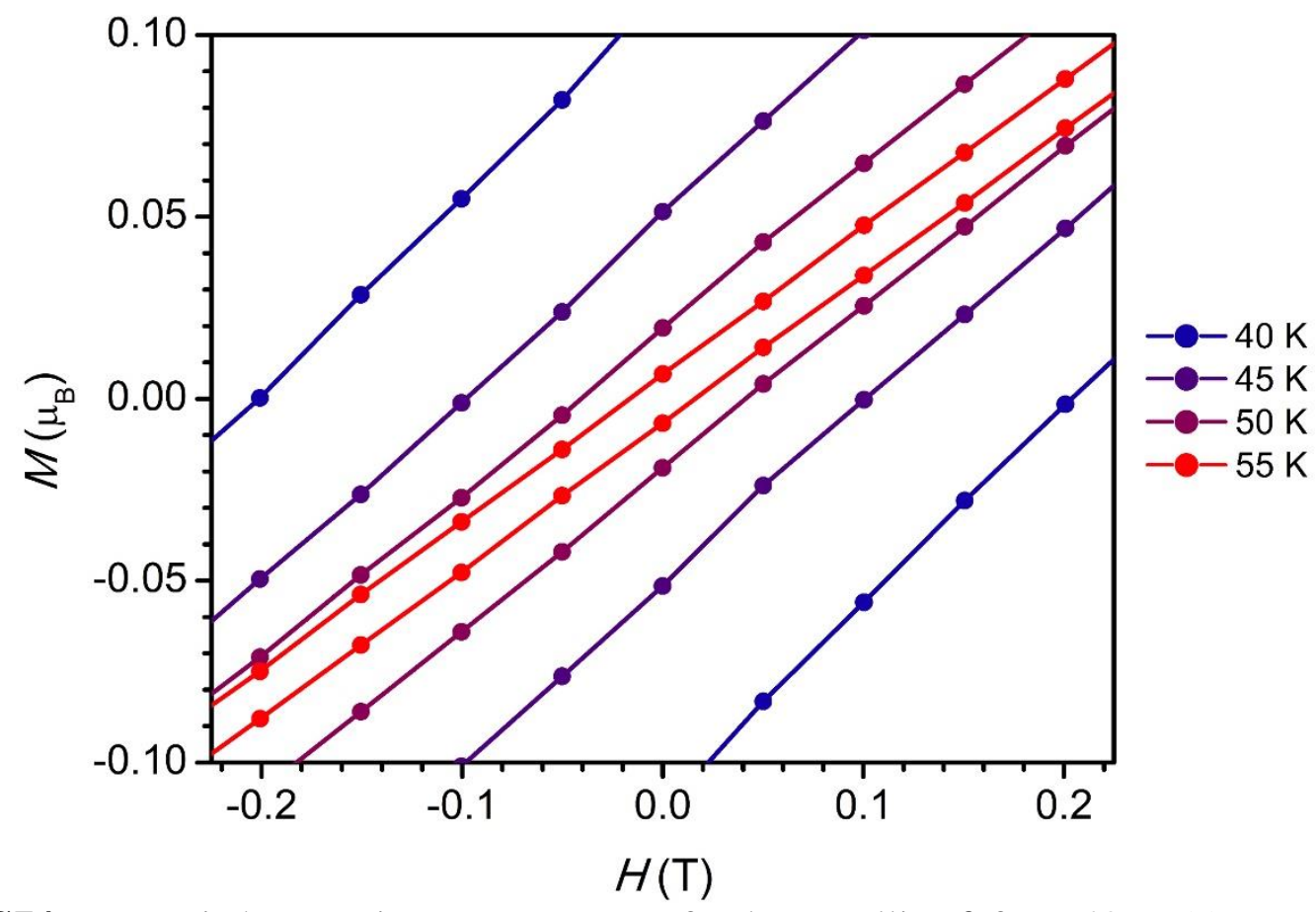

Figure S74. Magnetic hysteresis measurements of polycrystalline 2 from 40 to $55 \mathrm{~K}$ at a sweep rate of 14.7(1) mT/s for $H>2 \mathrm{~T}$ and 3.9(2) $\mathrm{mT} / \mathrm{s}$ for $H<2 \mathrm{~T}$.

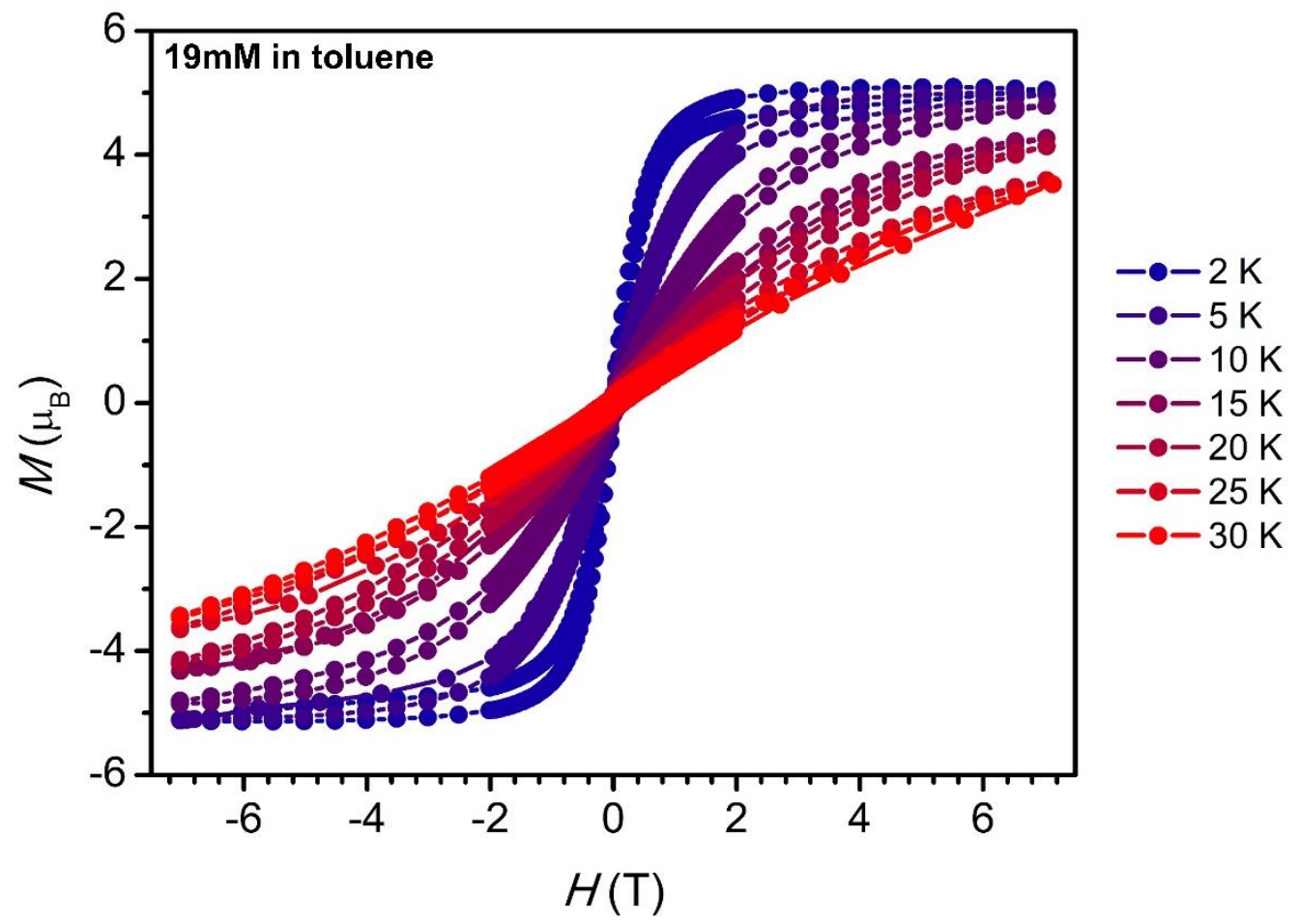

Figure S75. Magnetic hysteresis measurements of a $19 \mathrm{mM}$ solution of 2 in toluene from 2 to 30 $\mathrm{K}$ at a sweep rate of $14.7(1) \mathrm{mT} / \mathrm{s}$ for $H>2 \mathrm{~T}$ and 3.9(2) $\mathrm{mT} / \mathrm{s}$ for $H<2 \mathrm{~T}$. 


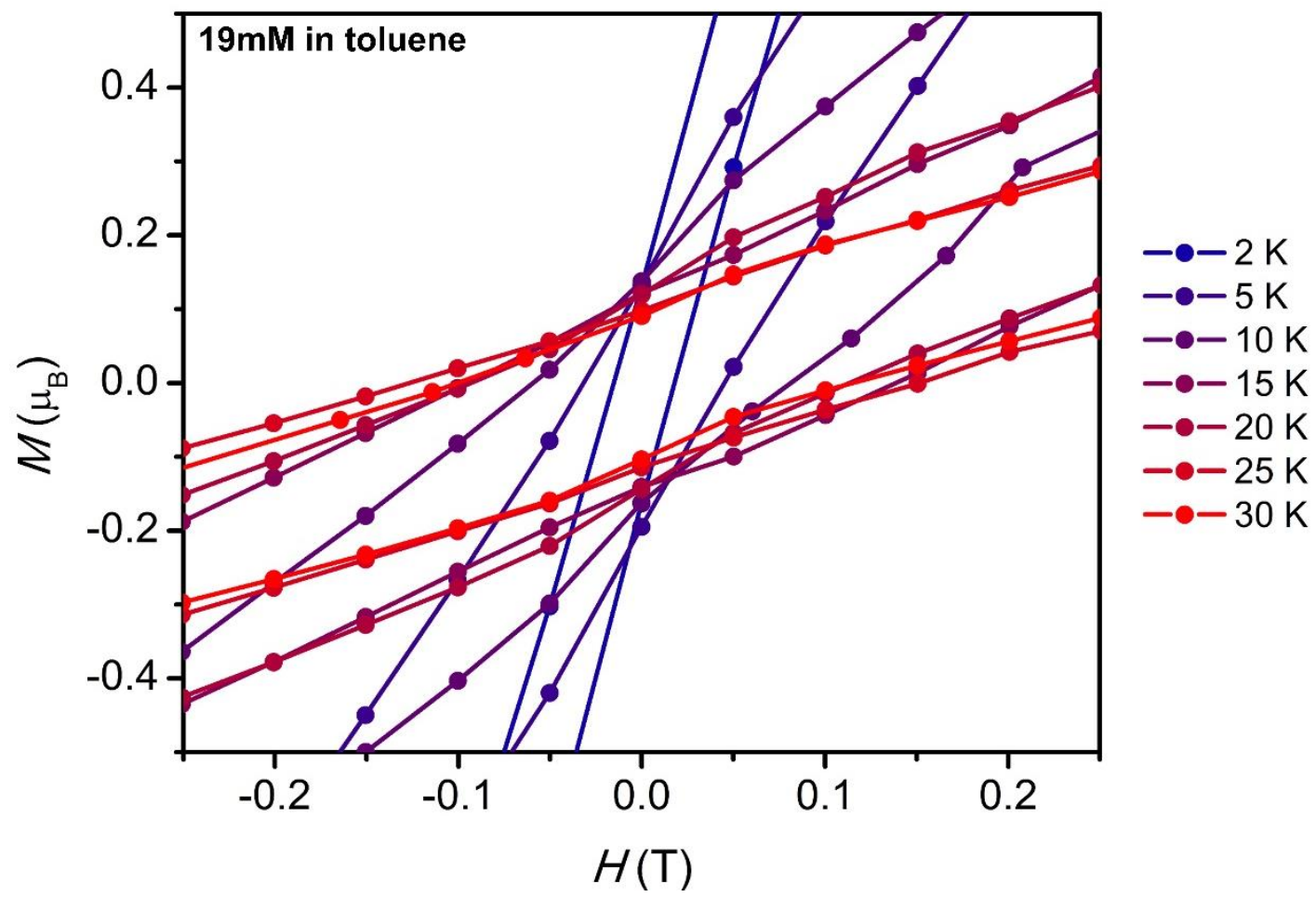

Figure S76. Magnetic hysteresis measurements of a $19 \mathrm{mM}$ solution of $\mathbf{2}$ in toluene from 2 to 30 $\mathrm{K}$ at a sweep rate of $14.7(1) \mathrm{mT} / \mathrm{s}$ for $H>2 \mathrm{~T}$ and 3.9(2) $\mathrm{mT} / \mathrm{s}$ for $H<2 \mathrm{~T}$.

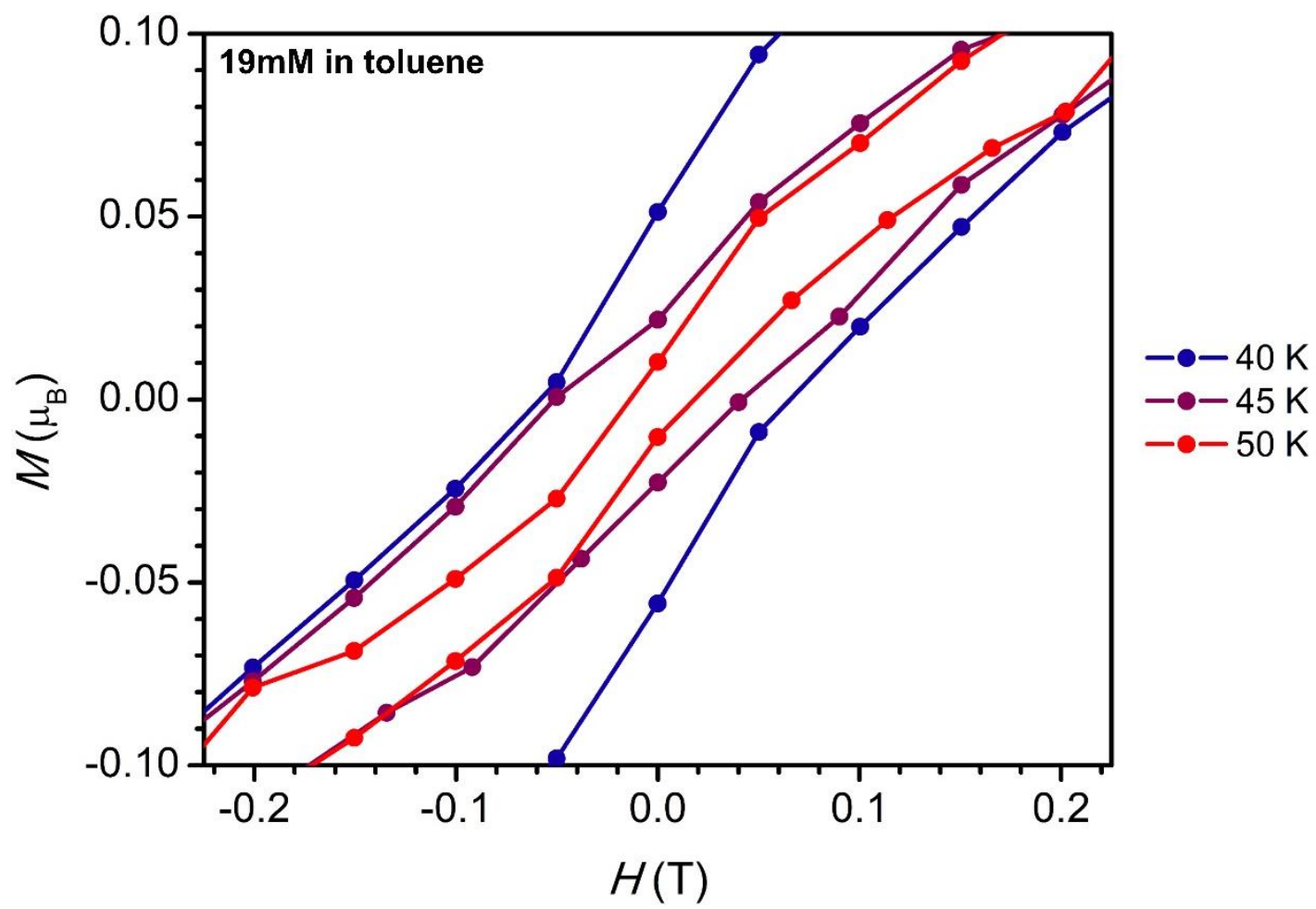

Figure S77. Magnetic hysteresis measurements of a $19 \mathrm{mM}$ solution of 2 in toluene from 40 to $50 \mathrm{~K}(5 \mathrm{~K}$ steps) at a sweep rate of $14.7(1) \mathrm{mT} / \mathrm{s}$ for $H>2 \mathrm{~T}$ and $3.9(2) \mathrm{mT} / \mathrm{s}$ for $H<2 \mathrm{~T}$. 


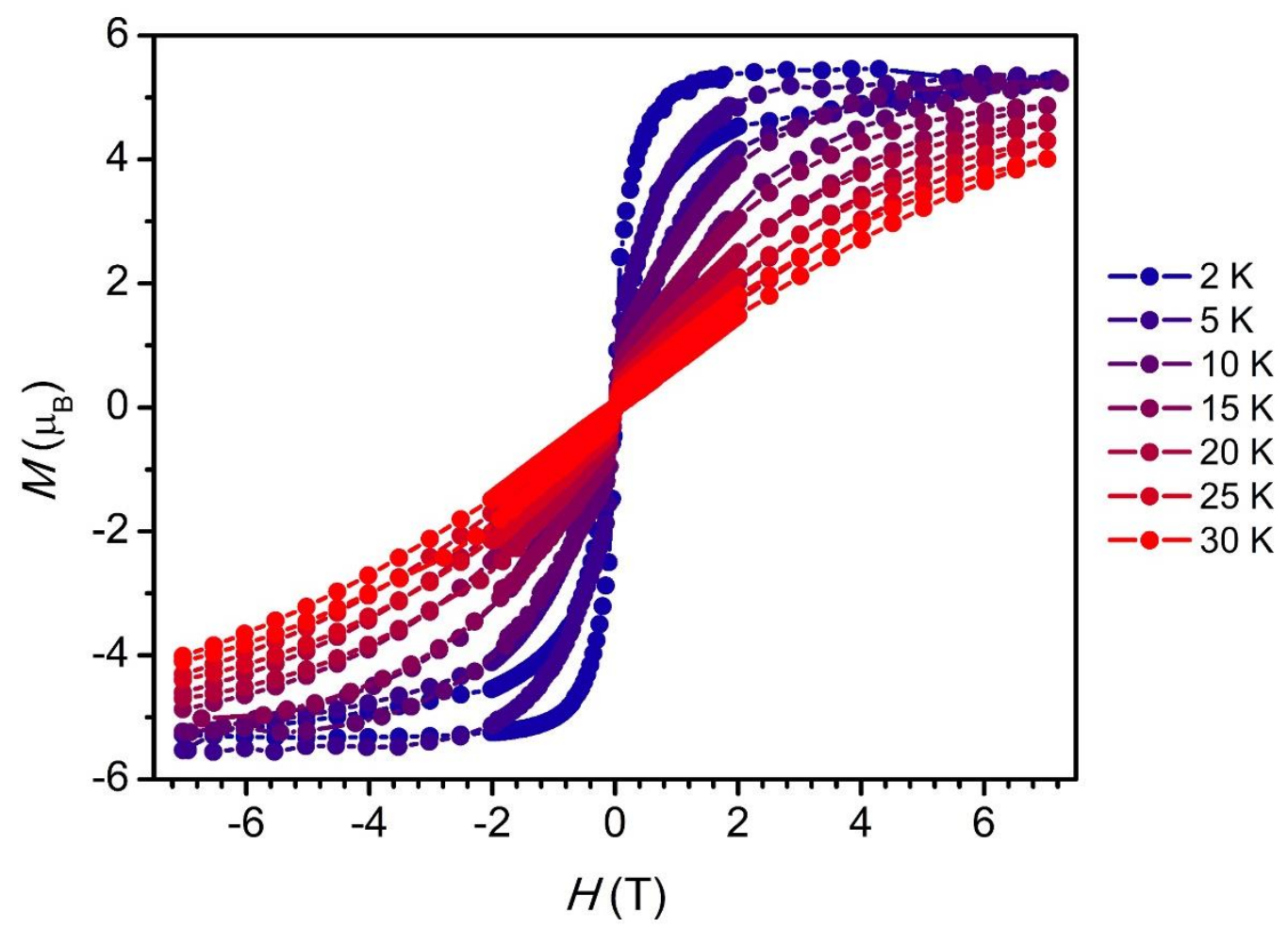

Figure S78. Magnetic hysteresis measurements of polycrystalline $\mathbf{3}$ from 2 to $30 \mathrm{~K}$ at a sweep rate of 14.7(1) $\mathrm{mT} / \mathrm{s}$ for $H>2 \mathrm{~T}$ and 3.9(2) $\mathrm{mT} / \mathrm{s}$ for $H<2 \mathrm{~T}$.

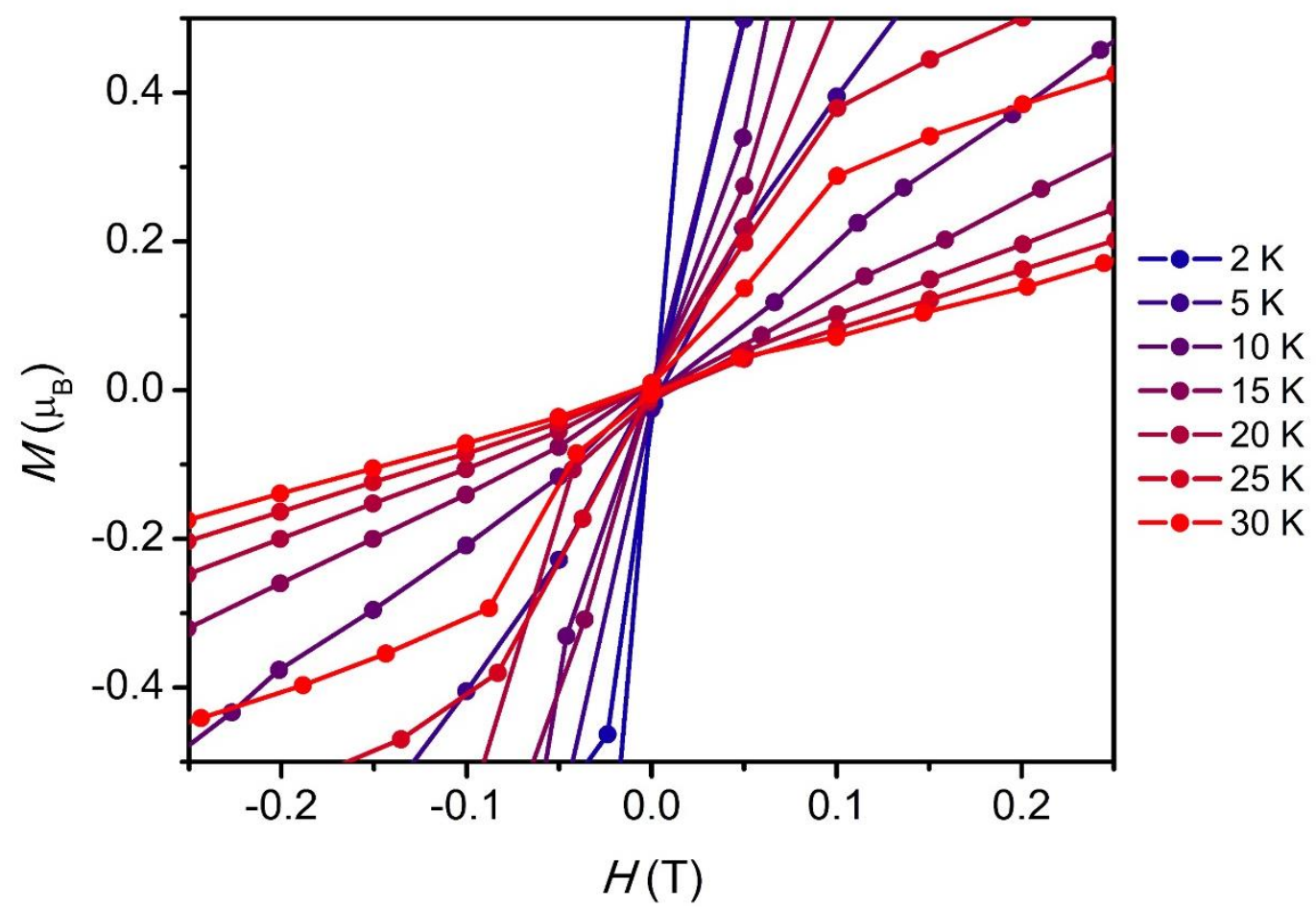

Figure S79. Magnetic hysteresis measurements of polycrystalline 3 from 2 to $30 \mathrm{~K}$ at a sweep rate of 14.7(1) mT/s for $H>2 \mathrm{~T}$ and 3.9(2) $\mathrm{mT} / \mathrm{s}$ for $H<2 \mathrm{~T}$. 


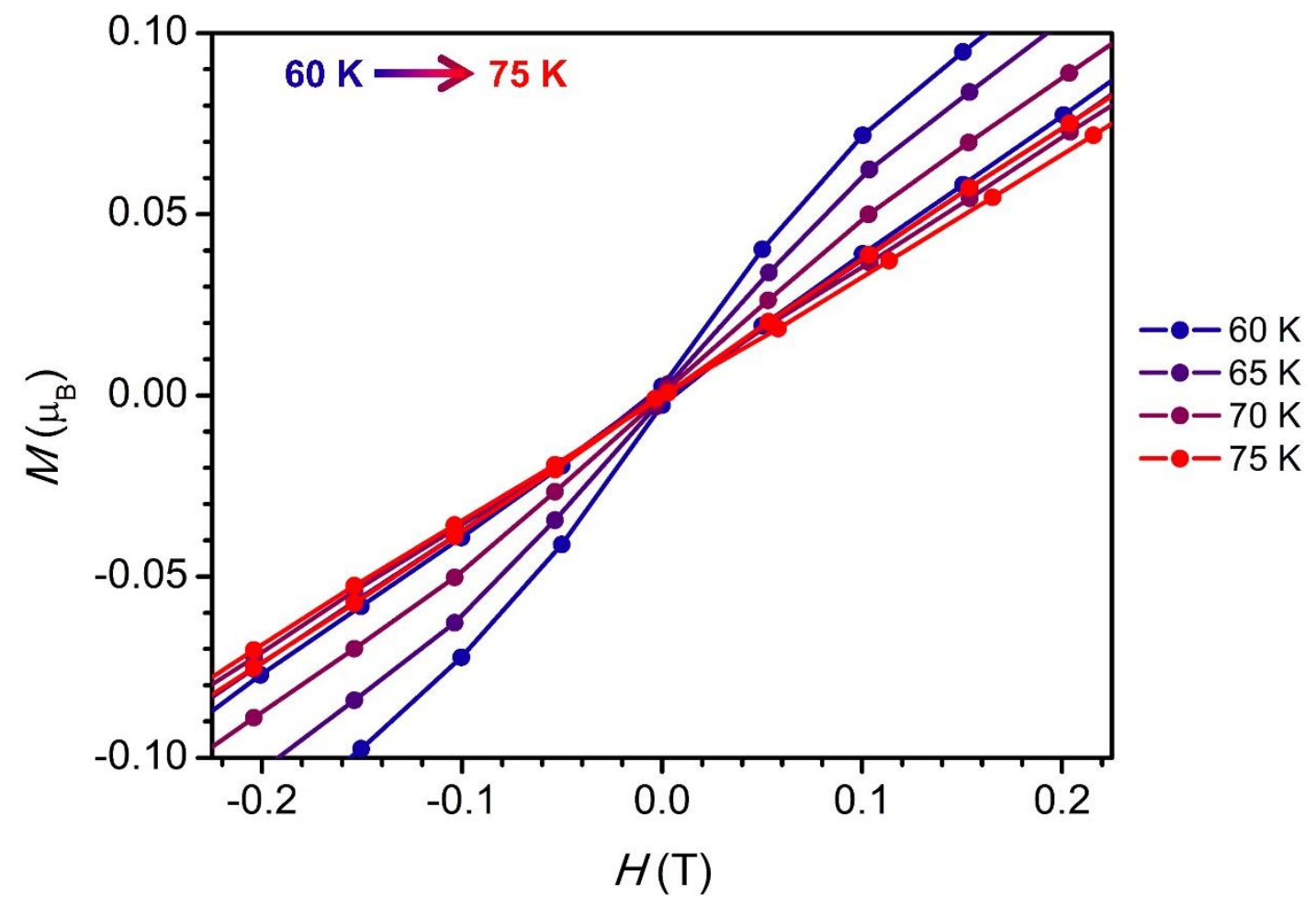

Figure S80. Magnetic hysteresis measurements of polycrystalline 3 from 60 to $75 \mathrm{~K}$ ( $5 \mathrm{~K}$ steps) at a sweep rate of 14.7(1) $\mathrm{mT} / \mathrm{s}$ for $H>2 \mathrm{~T}$ and 3.9(2) $\mathrm{mT} / \mathrm{s}$ for $H<2 \mathrm{~T}$.

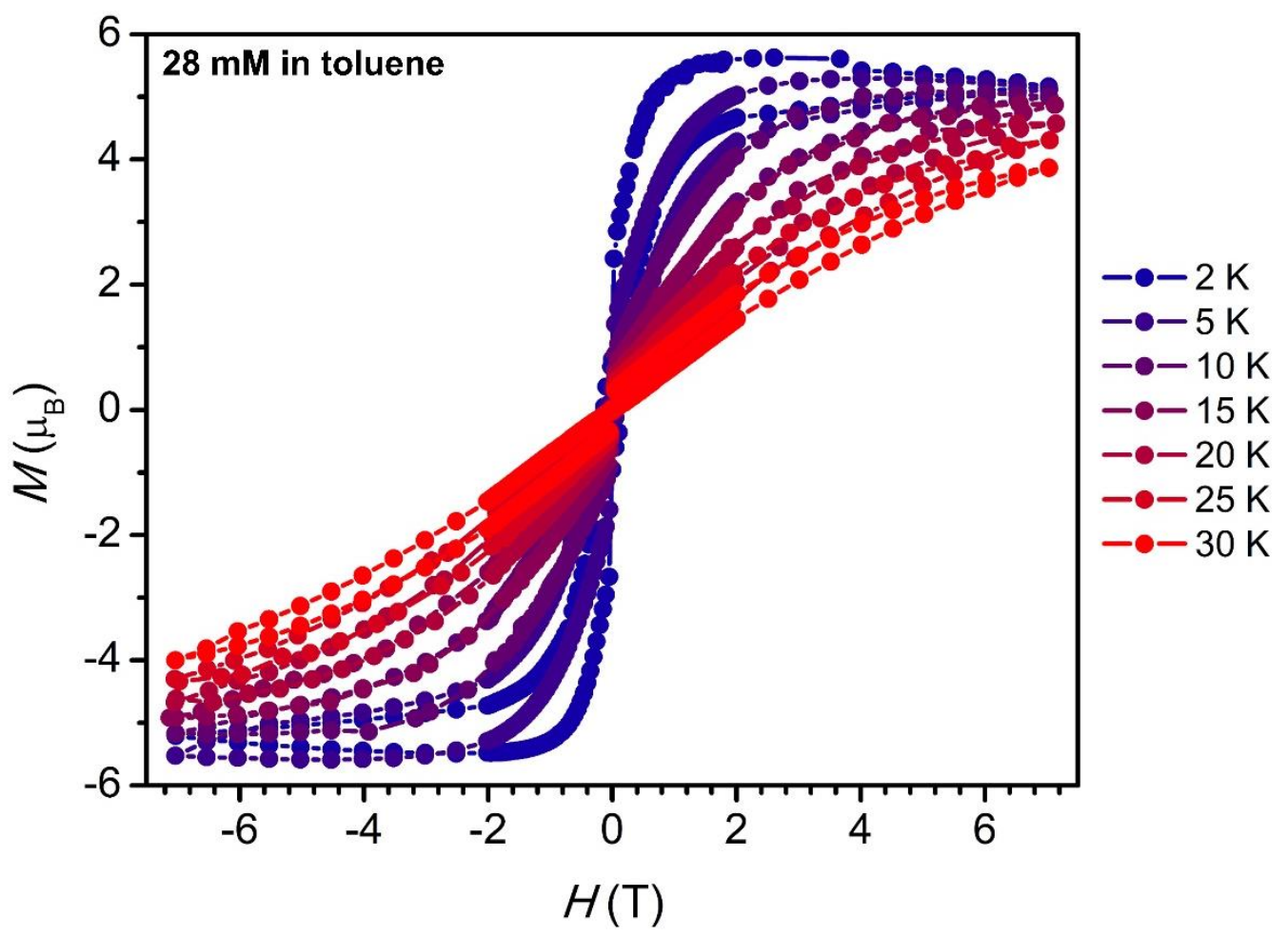

Figure S81. Magnetic hysteresis measurements of a $28 \mathrm{mM}$ solution of $\mathbf{3}$ in toluene from 2 to 30 $\mathrm{K}$ at a sweep rate of 14.7(1) $\mathrm{mT} / \mathrm{s}$ for $H>2 \mathrm{~T}$ and 3.9(2) $\mathrm{mT} / \mathrm{s}$ for $H<2 \mathrm{~T}$. 


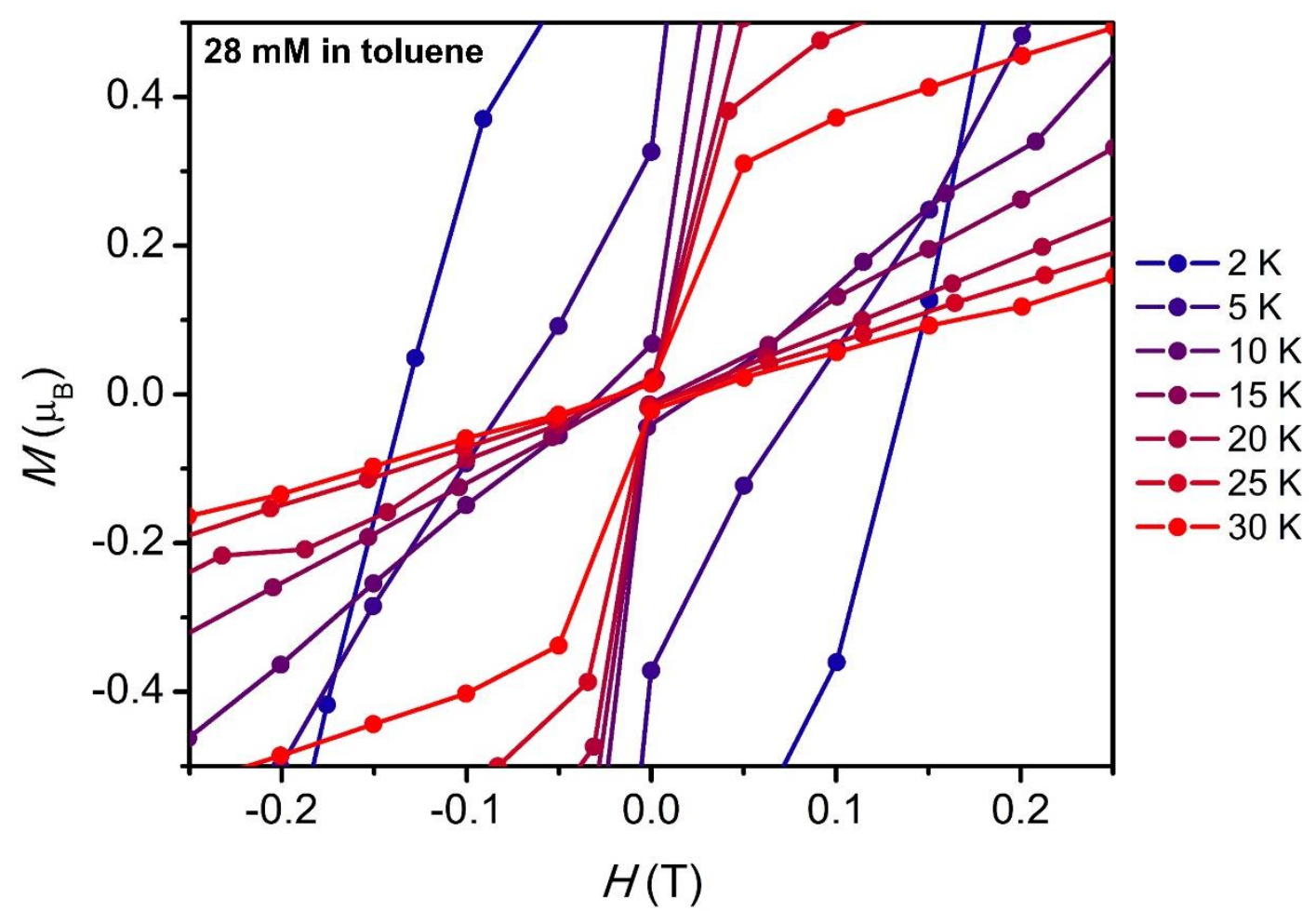

Figure S82. Magnetic hysteresis measurements of a $28 \mathrm{mM}$ solution of $\mathbf{3}$ in toluene from 2 to 30 $\mathrm{K}$ at a sweep rate of 14.7(1) $\mathrm{mT} / \mathrm{s}$ for $H>2 \mathrm{~T}$ and 3.9(2) $\mathrm{mT} / \mathrm{s}$ for $H<2 \mathrm{~T}$.

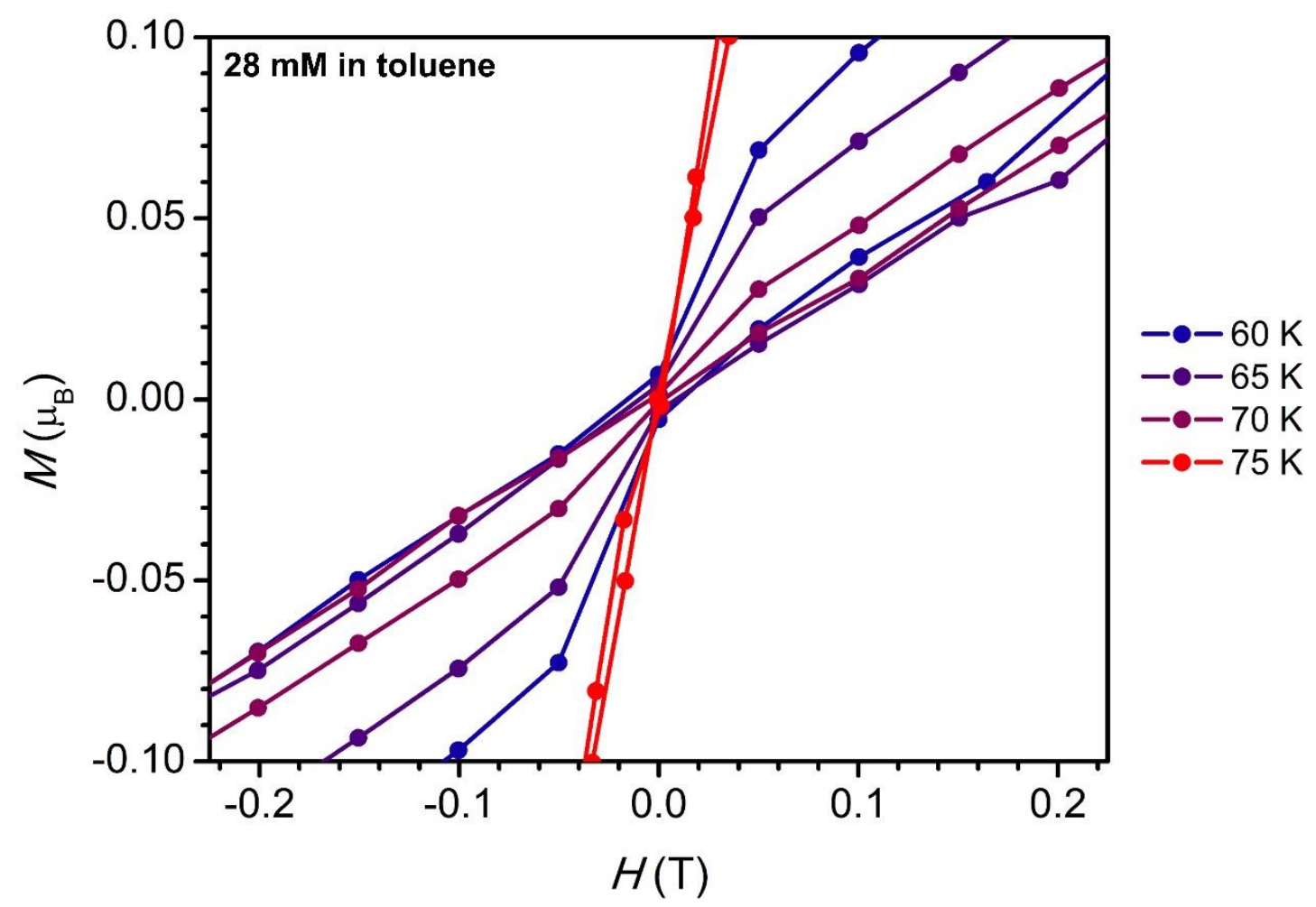

Figure S83. Magnetic hysteresis measurements of a $28 \mathrm{mM}$ solution of 3 in toluene from 60 to $75 \mathrm{~K}$ (5 K steps) at a sweep rate of $14.7(1) \mathrm{mT} / \mathrm{s}$ for $H>2 \mathrm{~T}$ and $3.9(2) \mathrm{mT} / \mathrm{s}$ for $H<2 \mathrm{~T}$. 


\section{Density Functional Theory Study}

Structures were taken from $\mathrm{x}$-ray crystallographic data of $\mathrm{Tb}\left(\mathrm{Cp}^{\mathrm{iPr} 5}\right)_{2}$ as a starting point for the computational studies of $\operatorname{Ln}\left(\mathrm{Cp}^{\mathrm{iPr} 5}\right)_{2}(\mathrm{Ln}=\mathrm{Dy}, \mathrm{Tb})$. These structures were optimized via Density Functional Theory (DFT) calculations to a geometry convergence tolerance of $10^{-4}$ a.u. and an electron density convergence tolerance of $10^{-7}$. Quadrature grids of size 4 were used for integral evaluation. The TPSS meta-generalized gradient approximation (meta-GGA) density functional was used for structural optimizations. ${ }^{8}$ Grimme's D3 dispersion correction and the resolution of the identity (RI-J) approximation were also used for computations. ${ }^{9,10}$ In all cases, structural convergence was verified via vibrational analysis. ${ }^{11}$

For $\operatorname{Ln}\left(\mathrm{Cp}^{\mathrm{iPr} 5}\right)_{2}(\mathrm{Ln}=\mathrm{Dy}, \mathrm{Tb})$ with $4 \mathrm{f}^{\mathrm{n}} 5 \mathrm{~d}^{1}$ valence configurations, both the double- $\zeta$ quality split valence basis set with polarization functions (def2-SV(P)) and triple- $\zeta$ quality basis set (def2TZVP) was used. ${ }^{12,13}$ In conjunction, an out-of-core quasi-relativistic def 2 effective core potential (ECP) for the Ln atoms was also used. ${ }^{14}$

To obtain the relative energy differences between $4 \mathrm{f}^{\mathrm{n}} 5 \mathrm{~d}^{1}$ valence configurations where the $\mathrm{d}$ electron is aligned or not aligned with unpaired f electrons, Fermi smearing was performed with a starting temperature between 2000-3000 K and ending temperature of 100-200 K with an annealing factor of 0.85 to obtain ground state occupations. The occupation numbers were constrained during Fermi smearing to ensure the desired spin multiplicity.

For study of the $4 \mathrm{f}^{\mathrm{n}+1}$ valence configurations, an $\mathrm{f}$-in-core quasi-relativistic ECP was used with the corresponding basis set (SCecp-mwb) on metal to ensure that the $\mathrm{Ln}=\mathrm{Dy}, \mathrm{Tb}$ atom housed all valence electrons in the $4 \mathrm{f}$ orbital. ${ }^{15,16}$ This ECP and basis was available from the StuttgartCologne basis set and pseudopotential library.

Files with optimized atomic coordinates for each configuration of $\operatorname{Ln}\left(\mathrm{Cp}^{\mathrm{iPr} 5}\right)_{2}(\mathrm{Ln}=\mathrm{Dy}, \mathrm{Tb})$ are attached to this SI, as well as the corresponding list of occupation numbers. Relative energies and metal-ligand distance are reported below.

Time-dependent DFT (TDDFT) calculations were performed for each electronic configuration to obtain excitation energies for $\operatorname{Ln}\left(\mathrm{Cp}^{\mathrm{iPr} 5}\right)_{2}(\mathrm{Ln}=\mathrm{Dy}, \mathrm{Tb}) .{ }^{17,18,19}$ These were done using the TPSS hybrid meta-GGA functional and the same choice of basis mentioned above. A convergence tolerance of $10^{-5}$ was used for the self-consistent procedure. Generation of visible absorption spectra was conducted with the lowest 10 excitations for each IR-active vibrational mode, or the lowest 55 if $\mathrm{C}_{1}$ symmetry was adopted. Visualizing $\mathrm{UV}-\mathrm{Vis}$ spectra was performed with a root mean square (RMS) width of $0.15 \mathrm{eV}$, a blue shift of $0.15 \mathrm{eV}$, and a vertical scaling of $0.5 \mathrm{eV}$. The blue-shift value is based on empirical observation of the performance of the TPSS functional.

Orbital visualization was performed with the VMD program with a contour value of $0.03 .{ }^{20}$ All calculations in this study were performed with the TURBOMOLE 7.3 quantum chemistry package. $^{21}$ 


\section{Effective Core Potential information}

Table S13. Number of electrons treated by f-out-of-core and f-in-core ECPs used with basis set.

\begin{tabular}{ccc}
\hline Ln & $\begin{array}{c}\text { f-out-of-core } \\
\boldsymbol{d e f 2 - S V ( P ) / T Z V P}\end{array}$ & $\begin{array}{c}\boldsymbol{f} \text {-in-core } \\
\text { SCecp-mwb }\end{array}$ \\
\hline $\mathrm{Dy}$ & 28 & 55 \\
\hline $\mathrm{Tb}$ & 28 & 56 \\
\hline
\end{tabular}

\section{Computed properties of $\mathrm{Dy}\left(\mathrm{Cp}^{\mathrm{iPr} 5}\right)_{2}$}

Optimized structures for $\mathrm{Dy}\left(\mathrm{Cp}^{\mathrm{iPr} 5}\right)_{2}$ were found to adopt both $\mathrm{D}_{5}$ and $\mathrm{S}_{10}$ point group symmetry. Comparison between the structures with both symmetries are included below. For $4 \mathrm{f}^{\mathrm{n}} 5 \mathrm{~d}^{1}$ configurations, further comparison was made between septet and quintet spin multiplicities which were obtained by flipping the spin of the $5 \mathrm{~d}^{1}$ electron. Term symbols denoting the symmetry of the ground state configuration state function were determined by subduction of the direct product representation of the molecular point groups (S10 and C1) spanned by all occupied molecular orbitals.

Table S14. Structural and electronic properties of $\mathrm{Dy}\left(\mathrm{Cp}^{\mathrm{iPr} 5}\right)_{2}$ calculated with def2-SV(P) basis set for $4 \mathrm{f}^{\mathrm{n}} 5 \mathrm{~d}^{1}$ configurations. The SCecp-mwb basis set on Dy is used for $4 \mathrm{f}^{\mathrm{n}+1}$ configurations.

\begin{tabular}{ccccc}
\hline Point Group & Term Symbol & Main Config. & Relative Energy $(\boldsymbol{e V})$ & Dy-Cp distance $(\mathbf{\AA})$ \\
\hline $\mathrm{S}_{10}$ & ${ }^{7} \mathrm{~A}_{\mathrm{g}}$ & $4 \mathrm{f}^{9} 5 \mathrm{~d}^{1}$ & 0 & 2.482 \\
\hline $\mathrm{S}_{10}$ & ${ }^{5} \mathrm{~A}_{\mathrm{g}}$ & $4 \mathrm{f}^{9} 5 \mathrm{~d}^{1}$ & 0.15 & 2.482 \\
\hline $\mathrm{S}_{10}$ & ${ }^{5} \mathrm{~A}_{\mathrm{u}}$ & {$\left[4 \mathrm{f}^{10}\right]^{*}$} & $-{ }^{*}$ & 2.574 \\
\hline $\mathrm{D}_{5}$ & ${ }^{7} \mathrm{~A}_{1}$ & $4 \mathrm{f}^{9} 5 \mathrm{~d}^{1}$ & 0.01 & 2.481 \\
\hline $\mathrm{D}_{5}$ & ${ }^{5} \mathrm{~A}_{1}$ & $4 \mathrm{f}^{9} 5 \mathrm{~d}^{1}$ & 0.22 & 2.493 \\
\hline $\mathrm{D}_{5}$ & ${ }^{5} \mathrm{~A}_{2}$ & {$\left[4 \mathrm{f}^{10}\right]$} & - & 2.547 \\
\hline
\end{tabular}

${ }^{\circ}$ Brackets around the electron configuration indicate that the electrons corresponding to the orbital are located inside the core of the ECP.

${ }^{* *}$ Relative energy is not compared for the $4 \mathrm{f}^{\mathrm{n}+1}$ case as the number of explicitly included electrons, and thus the computed energy systematically differs.

Table S15. Structural and electronic properties of $\mathrm{Dy}\left(\mathrm{Cp}^{\mathrm{iPr} 5}\right)_{2}$ with def2-TZVP basis set for $4 \mathrm{f}^{\mathrm{n}}$ $5 \mathrm{~d}^{1}$ configurations. The SCecp-mwb basis set on Dy is used for $4 \mathrm{f}^{\mathrm{n}+1}$ configurations.

\begin{tabular}{ccccc}
\hline Point Group & Term Symbol & Main Config. & Relative Energy $(\boldsymbol{e V})$ & Dy-Cp distance $(\mathbf{A})$ \\
\hline $\mathrm{S}_{10}$ & ${ }^{7} \mathrm{~A}_{\mathrm{g}}$ & $4 \mathrm{f}^{9} 5 \mathrm{~d}^{1}$ & 0 & 2.487 \\
\hline $\mathrm{S}_{10}$ & ${ }^{5} \mathrm{~A}_{\mathrm{g}}$ & $4 \mathrm{f}^{9} 5 \mathrm{~d}^{1}$ & 0.13 & 2.488 \\
\hline $\mathrm{S}_{10}$ & ${ }^{5} \mathrm{~A}_{\mathrm{u}}$ & {$\left[4 \mathrm{f}^{10}\right]$} & - & 2.561 \\
\hline $\mathrm{D}_{5}$ & ${ }^{7} \mathrm{~A}_{1}$ & $4 \mathrm{f}^{9} 5 \mathrm{~d}^{1}$ & 0.01 & 2.489 \\
\hline $\mathrm{D}_{5}$ & ${ }^{5} \mathrm{~A}_{1}$ & $4 \mathrm{f}^{9} 5 \mathrm{~d}^{1}$ & 0.15 & 2.484 \\
\hline $\mathrm{D}_{5}$ & ${ }^{5} \mathrm{~A}_{2}$ & {$\left[4 \mathrm{f}^{10}\right]$} & - & 2.560 \\
\hline
\end{tabular}


Comparison of Dy-Cp distances from DFT optimized Dy $\left(C \mathrm{p}^{\mathrm{iPr} 5}\right)_{2}$ structures between the $4 \mathrm{f}^{\mathrm{n}} 5 \mathrm{~d}^{1}$ and $4 \mathrm{f}^{\mathrm{n}+1}$ electronic configuration support the experimentally observed lengthening on the order of $5.0 \times 10^{-2} \AA$. These results are in line with previous studies and reinforces the observed $4 \mathrm{f}^{\mathrm{n}} 5 \mathrm{~d}^{1}$ configuration (see full text). ${ }^{22}$

Energy differences between the septet and quintet spin multiplicities of the $4 \mathrm{f}^{\mathrm{n}} 5 \mathrm{~d}^{1}$ electron configurations were found to be roughly $0.15 \mathrm{eV}$, which may indicate a preference towards the high-spin state in the scalar-relativistic picture. Energy differences between $\mathrm{Dy}\left(\mathrm{Cp}{ }^{\mathrm{iPr} 5}\right)_{2}$ in the $\mathrm{D}_{5}$ and $\mathrm{S}_{10}$ point group fall within $0.01 \mathrm{eV}$, and thus are considered roughly isoenergetic.

Table S16. Natural population analysis conducted for the ${ }^{7} \mathrm{~A}_{1}$ term symbol for $\mathrm{Dy}\left(\mathrm{C} \mathrm{p}^{\mathrm{iPr} 5}\right)_{2}{ }^{23}$

\begin{tabular}{ccccc}
\hline Atom & $\boldsymbol{n}(\boldsymbol{s})$ & $\boldsymbol{n}(\boldsymbol{p})$ & $\boldsymbol{n}\left(\boldsymbol{d} \boldsymbol{z}^{2}\right)$ & $\boldsymbol{n}(\boldsymbol{d} \text { rest })^{*}$ \\
\hline Dy & 0.6947 & 0 & 0.1816 & 0 \\
\hline $\mathrm{C}^{* *}$ & 0.0068 & 0.0528 & 0 & 0
\end{tabular}

${ }^{*}$ Includes natural occupancies from all other d orbitals besides $\mathrm{z}^{2}$

** Represents summed contribution from the 10 Carbon atoms located on the two Cp rings.

Calculation of natural orbital populations for the HOMO revealed significant $6 \mathrm{~s}$ mixing and small contributions originating from ligand $\mathrm{p}$ orbitals. Although the ratio of $\mathrm{s}$ to $\mathrm{d}$ character seems high, population analysis schemes often largely overestimate the relative s contribution. Mixing from ligand orbitals lend to stabilization of the $5 \mathrm{dz}^{2}$ orbital as the HOMO, and the ordering of $5 \mathrm{~d}$ energies thus differs from the traditional textbook picture. The present results are however supported both from photoionization profiles and more recent computational studies of ferrocene. ${ }^{24,25}$

Table S17. Comparison of significant electronic excitations (> 0.0015 osc str.) for S10 term symbols computed with TDDFT and the TPSS functional. Blue shifted results are also included in parentheses. For these calculations, a triple- $\zeta$ quality basis set (def2-TZVP) was used for the metal and a double- $\zeta$ quality basis set (def2-SV(P)) for the ligand atoms.

\begin{tabular}{|c|c|c|c|c|c|}
\hline $\begin{array}{c}\text { Term } \\
\text { Symbol }\end{array}$ & $\begin{array}{l}\text { Wavelength } \\
\text { (nm) }\end{array}$ & $\begin{array}{l}\text { Oscillator } \\
\text { Str. }\end{array}$ & $\begin{array}{c}\text { Occ-virt orbital } \\
\text { transition }\end{array}$ & $\begin{array}{c}\text { Occupied } \\
\text { (virtual) } \\
\text { energy }(e V)\end{array}$ & $\begin{array}{c}\text { Type of } \\
\text { excitation }\end{array}$ \\
\hline \multirow[t]{7}{*}{${ }^{7} \mathrm{~A}_{\mathrm{g}}$} & $739.0(678.4)$ & 0.00641 & $32 \mathrm{e}_{2 \mathrm{u}} \rightarrow 34 \mathrm{e}_{1 \mathrm{~g}}$ & $-2.43(-0.65)$ & $4 f \rightarrow 5 d$ \\
\hline & $403.8(385.0)$ & 0.01505 & $38 \mathrm{e}_{1 \mathrm{u}} \rightarrow 34 \mathrm{e}_{1 \mathrm{~g}}$ & $-4.41(-1.32)$ & LMCT $^{*}$ \\
\hline & $370.6(354.7)$ & 0.00539 & $34 \mathrm{e}_{2 \mathrm{u}} \rightarrow 34 \mathrm{e}_{1 \mathrm{~g}}$ & $-4.80(-1.32)$ & $4 \mathrm{f} \rightarrow 5 \mathrm{~d}$ \\
\hline & $366.9(351.3)$ & 0.00387 & $36 \mathrm{e}_{1 \mathrm{u}} \rightarrow 20 \mathrm{a}_{\mathrm{g}}$ & $-4.47(-1.03)$ & LMCT \\
\hline & $327.5(315.0)$ & 0.00256 & $38 \mathrm{e}_{1 \mathrm{u}} \rightarrow 21 \mathrm{a}_{\mathrm{g}}$ & $-4.77(-0.08)$ & LMCT \\
\hline & $325.1(312.8)$ & 0.00855 & $34 \mathrm{e}_{2 \mathrm{~g}} \rightarrow 38 \mathrm{e}_{1 \mathrm{u}}$ & $-4.85(-1.01)$ & LMCT \\
\hline & $318.5(306.7)$ & 0.04980 & & $-4.47(-0.65)$ & LMCT \\
\hline
\end{tabular}




\begin{tabular}{|c|c|c|c|c|c|}
\hline & 294.0 & 0.01806 & $\begin{array}{c}36 \mathrm{e}_{1 \mathrm{u}} \rightarrow 34 \mathrm{e}_{1 \mathrm{~g}} \\
(46 \%) \\
38 \mathrm{e}_{1 \mathrm{u}} \rightarrow 21 \mathrm{ag}_{\mathrm{g}} \\
(50 \%)\end{array}$ & $-4.41(-0.54)$ & MLCT $^{* *}$ \\
\hline & $292.2(282.2)$ & 0.00480 & $\begin{array}{l}20 \mathrm{a}_{\mathrm{g}} \rightarrow 40 \mathrm{e}_{1 \mathrm{u}} \\
32 \mathrm{e}_{2 \mathrm{u}} \rightarrow 38 \mathrm{e}_{1 \mathrm{~g}}\end{array}$ & $-2.43(1.85)$ & LMCT \\
\hline \multirow[t]{6}{*}{${ }^{5} \mathrm{Ag}_{\mathrm{g}}$} & $937.9(842.3)$ & 0.00575 & $32 \mathrm{e}_{2 \mathrm{u}} \rightarrow 34 \mathrm{e}_{1 \mathrm{~g}}$ & $-3.48(-0.72)$ & $4 \mathrm{f} \rightarrow 5 \mathrm{~d}$ \\
\hline & $419.3(399.1)$ & 0.00285 & $\begin{array}{r}38 \mathrm{e}_{1 \mathrm{u}} \rightarrow 20 \mathrm{a}_{\mathrm{g}} \\
\quad(44 \%) \\
34 \mathrm{e}_{2 \mathrm{~g}} \rightarrow 19 \mathrm{a}_{\mathrm{u}} \\
(45 \%)\end{array}$ & $\begin{array}{l}-4.39(-1.39) \\
-4.88(-1.92)\end{array}$ & LMCT \\
\hline & $\begin{array}{c}345.8 \\
(331.9)\end{array}$ & 0.05037 & $\begin{array}{c}38 \mathrm{e}_{1 \mathrm{u}} \rightarrow 34 \mathrm{e}_{1 \mathrm{~g}} \\
(42 \%) \\
36 \mathrm{e}_{1 \mathrm{u}} \rightarrow 34 \mathrm{e}_{1 \mathrm{~g}} \\
(41 \%)\end{array}$ & $\begin{array}{l}-4.39(-0.94) \\
-4.49(-1.07)\end{array}$ & LMCT \\
\hline & $334.0(321.0)$ & 0.01010 & $34 \mathrm{e}_{2 \mathrm{~g}} \rightarrow 38 \mathrm{e}_{1 \mathrm{u}}$ & $-4.88(-1.13)$ & LMCT \\
\hline & $312.7(301.3)$ & 0.02200 & $20 \mathrm{a}_{\mathrm{g}} \rightarrow 40 \mathrm{e}_{1 \mathrm{u}}$ & $-3.66(0.32)$ & MLCT \\
\hline & $294.1(284.0)$ & 0.09671 & $20 \mathrm{a}_{\mathrm{g}} \rightarrow 20 \mathrm{a}_{\mathrm{u}}$ & $-3.66(0.55)$ & MLCT \\
\hline \multirow[t]{2}{*}{${ }^{5} \mathrm{~A}_{\mathrm{u}}$} & $383.5(368.3)$ & 0.00829 & $34 \mathrm{e}_{1 \mathrm{u}} \rightarrow 18 \mathrm{a}_{\mathrm{g}}$ & $-4.28(-1.02)$ & LMCT \\
\hline & $313.7(302.2)$ & 0.06953 & $34 \mathrm{e}_{1 \mathrm{u}} \rightarrow 32 \mathrm{e}_{1 \mathrm{~g}}$ & $-4.28(-0.49)$ & LMCT \\
\hline
\end{tabular}

*Ligand to metal charge transition

** Metal to ligand charge transition 


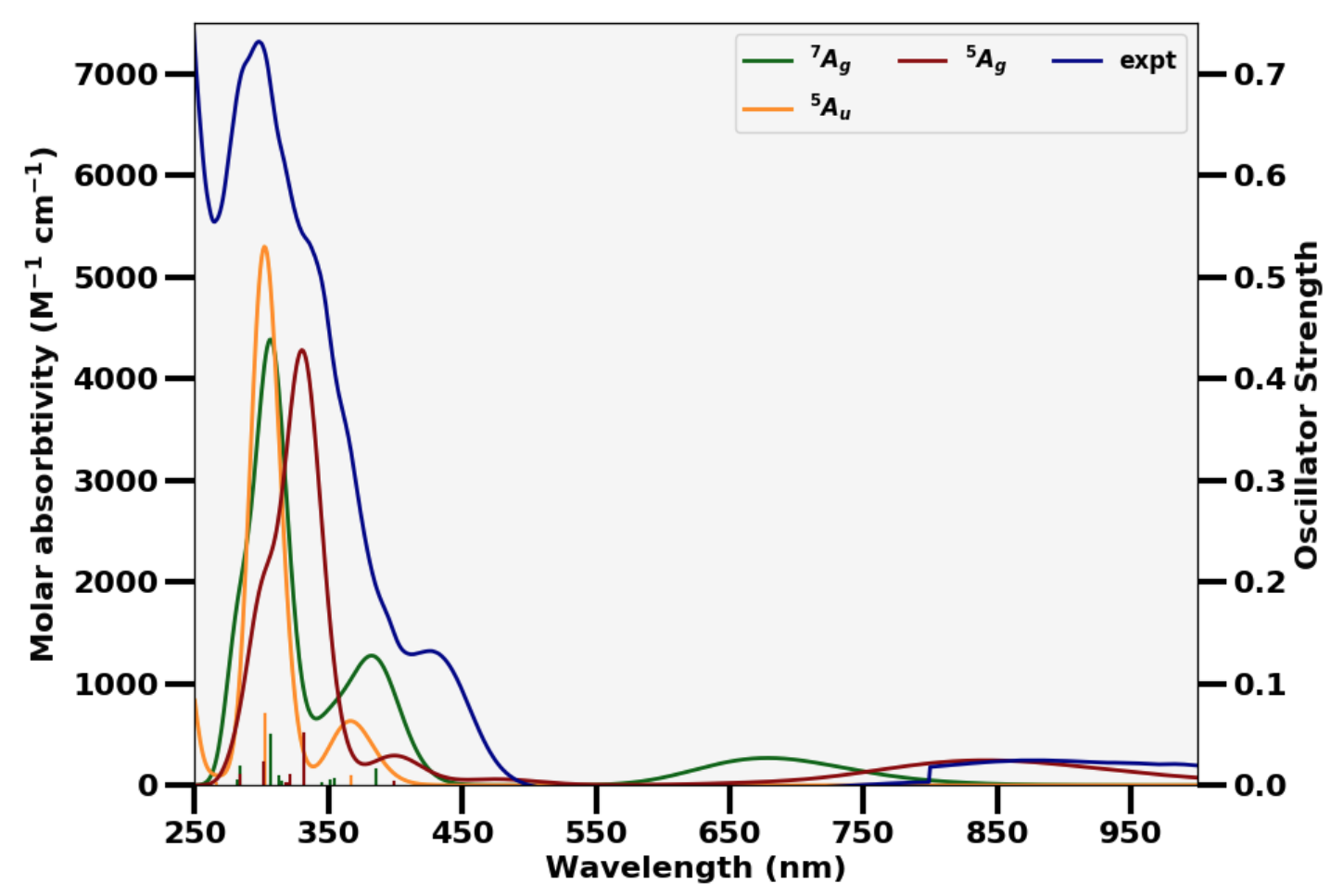

Figure S84. Computed UV-Vis spectra for $\mathrm{Dy}\left(\mathrm{Cp}^{\mathrm{iPr} 5}\right)_{2}$ with corresponding term symbols in the $\mathrm{S}_{10}$ point group. A RMS width of $0.15 \mathrm{eV}$, a blue shift of $0.15 \mathrm{eV}$, and a vertical scaling of 0.5 $\mathrm{eV}$ was used. Information regarding individual excitations can be found in Table S17.

Intense peaks found at around $430 \mathrm{~nm}$ and $296 \mathrm{~nm}$ in the experimental spectra were predicted as blue-shifted ligand-metal charge transfer excitations through DFT. Between predicted spectra for the ${ }^{7} \mathrm{~A}_{\mathrm{g}},{ }^{5} \mathrm{~A}_{\mathrm{g}}$, and ${ }^{5} \mathrm{~A}_{\mathrm{u}}$ term symbols, the two aforementioned peaks occurred in roughly the same locations (although the ${ }^{5} \mathrm{Ag}_{\mathrm{g}}$ spectra was blue-shifted away from the other two). Broad peaks near the red end of the visible spectrum were found around $680 \mathrm{~nm}$ and $840 \mathrm{~nm}$ for the ${ }^{7} \mathrm{Ag}_{\mathrm{g}}$ and ${ }^{5} \mathrm{Ag}_{\mathrm{g}}$ terms, which were predicted to be due to a $4 \mathrm{f}$ to $5 \mathrm{~d}$ excitation. This peak is notably absent in the ${ }^{5} \mathrm{~A}_{\mathrm{u}}$ term, possibly due to the $5 \mathrm{dz}^{2}$ orbital sitting further above the $4 \mathrm{f}$ orbitals in energy for the $4 \mathrm{f}^{\mathrm{n}+1}$ configuration. These peaks may correspond to that located around $850 \mathrm{~nm}$ from the experimentally obtained spectra, and could thus lend further credibility to identifying the suspected $4 \mathrm{f}^{\mathrm{n}} 5 \mathrm{~d}^{1}$ configuration. 
Table S18. Computed metal-centered $4 \mathrm{f}$ and $5 \mathrm{~d}$ molecular orbitals and energies (in $\mathrm{eV}$ ) for term symbol ${ }^{7} \mathrm{~A}_{1}$ with def2-TZVP basis. The other $4 \mathrm{f}^{\mathrm{n}} 5 \mathrm{~d}^{1}$ terms in both $\mathrm{S} 10$ and D5 yield nearly identical MOs.

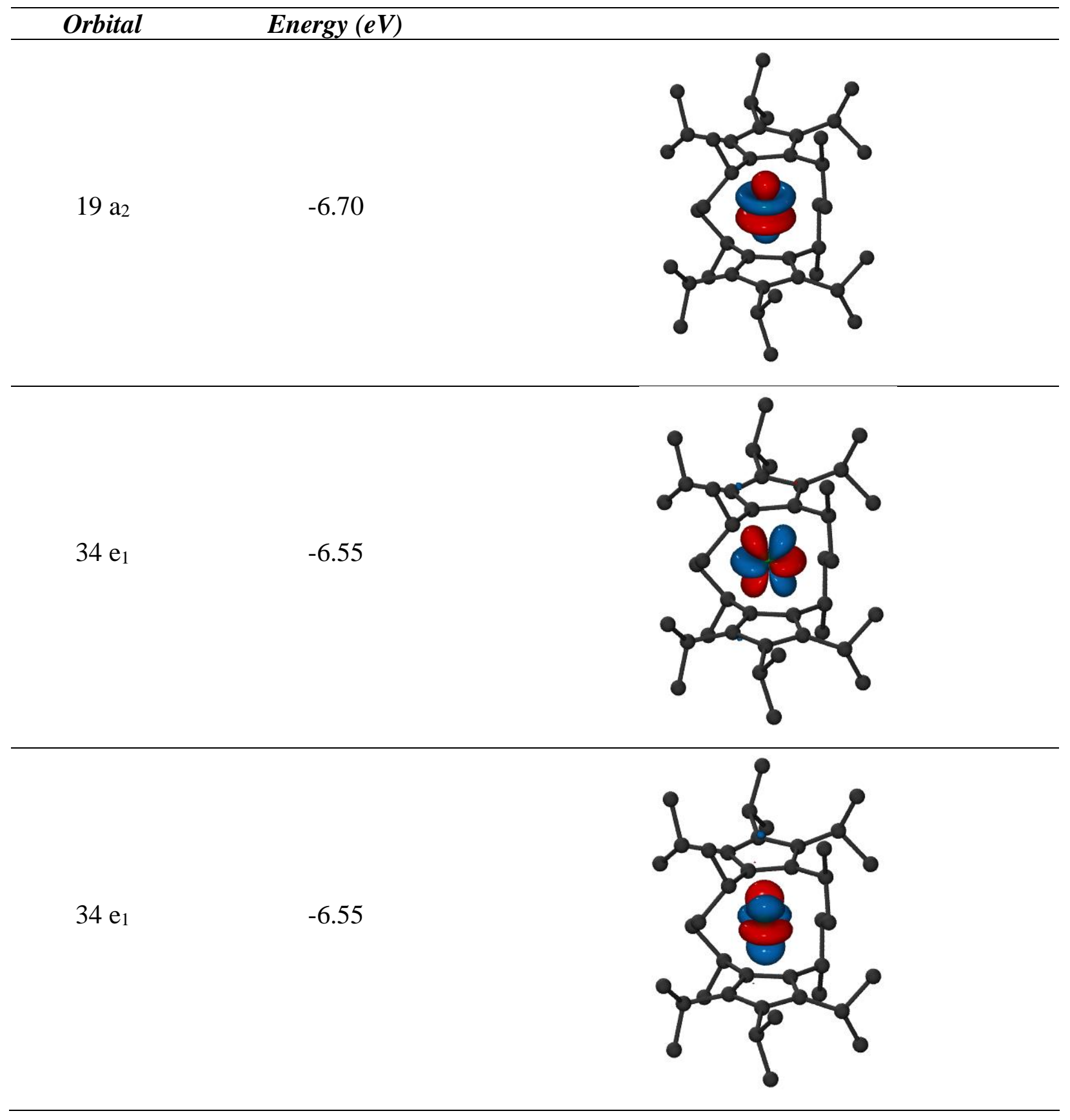




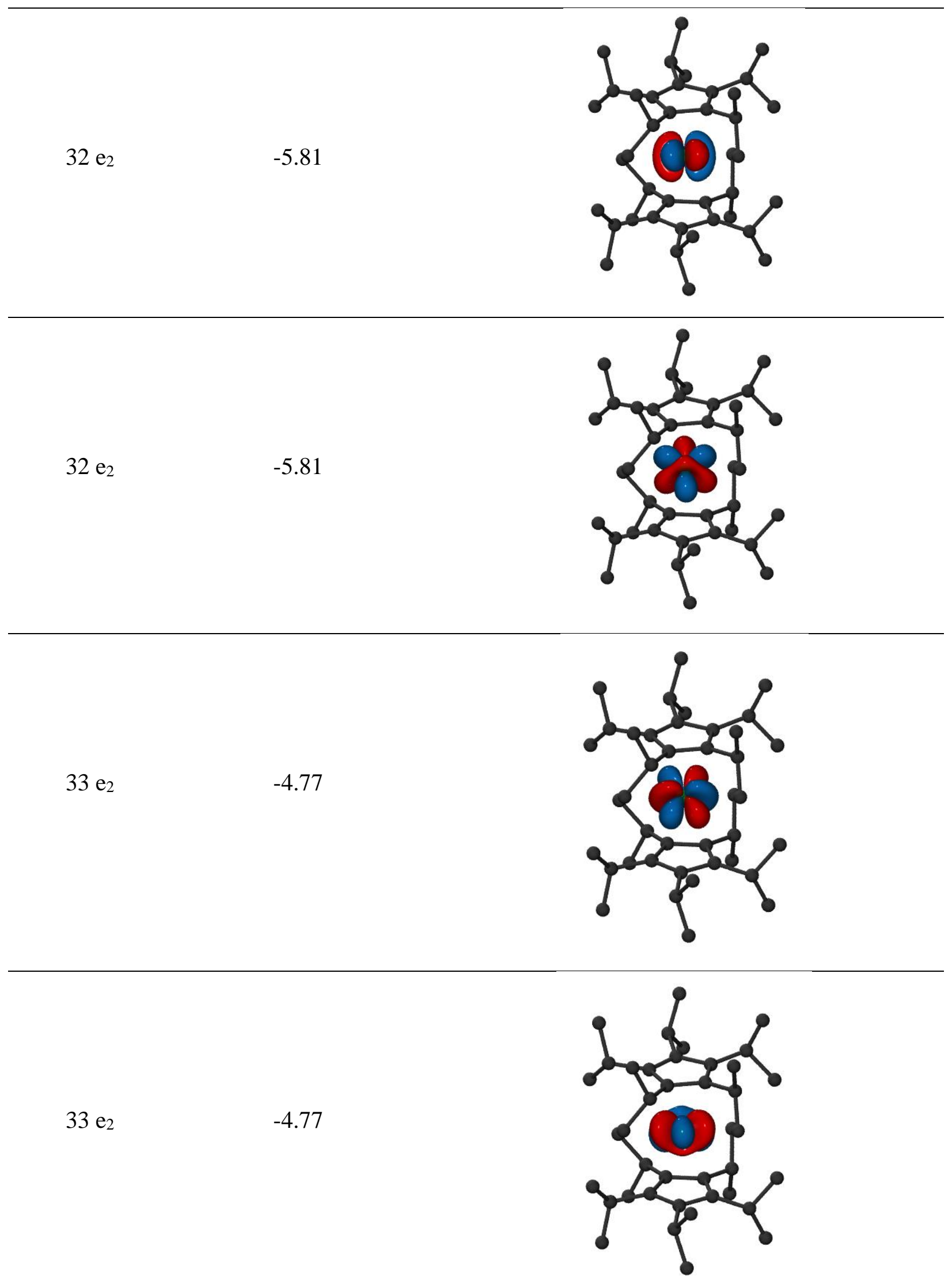




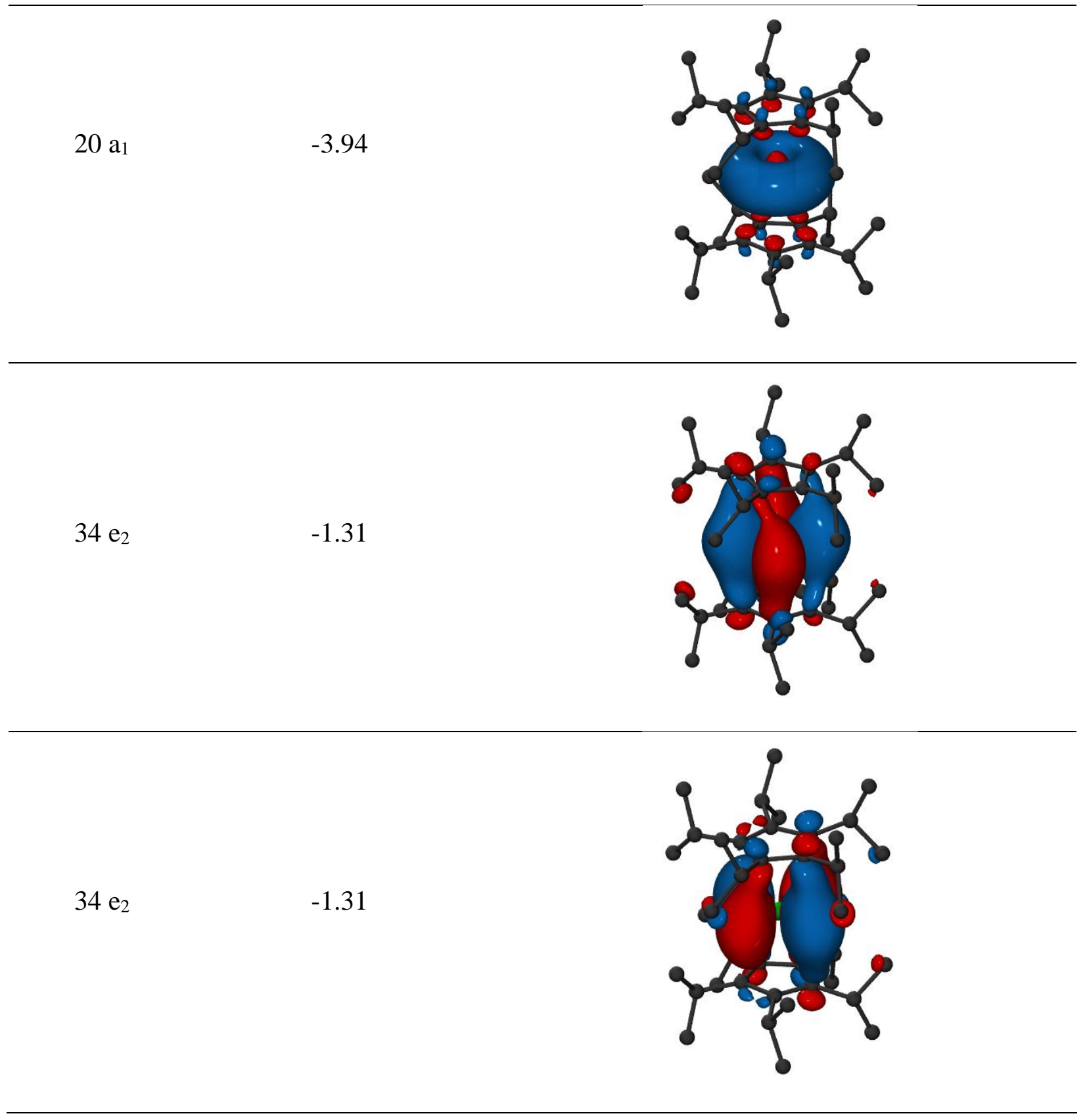


$40 \mathrm{e}_{1}$

1.60

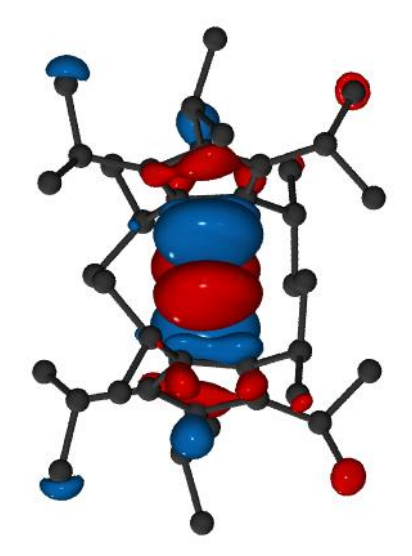

$40 \mathrm{e}_{1}$

1.60

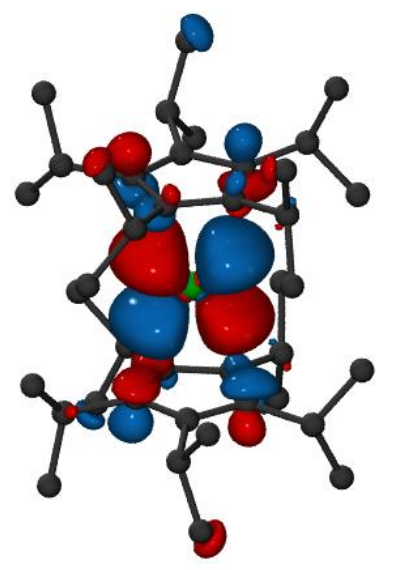




\section{Computed properties of $\mathrm{Tb}\left(\mathrm{Cp}^{\mathrm{iPr} 5}\right)_{2}$}

Optimized geometries for $\mathrm{Tb}\left(\mathrm{Cp}^{\mathrm{iPr} 5}\right)_{2}$ were found to have $\mathrm{C}_{1}$ symmetry, which differs from the $\mathrm{Dy}\left(\mathrm{Cp}^{\mathrm{iPr} 5}\right)_{2}$ complex above. This is possibly due to the observance of Jahn-Teller effects from partial occupation of degenerate e orbitals in the S10 and D5 symmetries.

Table S19. Structural and electronic properties of $\mathrm{Tb}\left(\mathrm{Cp}^{\mathrm{iPr} 5}\right)_{2}$ with def2-SV(P) basis set for $4 \mathrm{f}^{\mathrm{n}}$ $5 \mathrm{~d}^{1}$ configurations and SCecp-mwb basis set for $4 \mathrm{f}^{\mathrm{n}+1}$ configurations.

\begin{tabular}{ccccc}
\hline Point Group & Term Symbol & Main Config. & Relative Energy $(\boldsymbol{e V})$ & Tb-Cp distance $(\AA)$ \\
\hline $\mathrm{C}_{1}$ & ${ }^{8} \mathrm{~A}$ & $4 \mathrm{f}^{8} 5 \mathrm{~d}^{1}$ & 0 & 2.492 \\
\hline $\mathrm{C}_{1}$ & ${ }^{6} \mathrm{~A}$ & $4 \mathrm{f}^{8} 5 \mathrm{~d}^{1}$ & 0.15 & 2.476 \\
\hline $\mathrm{C}_{1}$ & ${ }^{6} \mathrm{~A}$ & {$\left[4 \mathrm{f}^{9}\right]^{*}$} & - & 2.600
\end{tabular}

*As before, brackets around the electron configuration indicate that the electrons corresponding to the orbital are located inside the core of the ECP.

Table S20. Structural and electronic properties of $\mathrm{Tb}\left(\mathrm{Cp}^{\mathrm{iPr} 5}\right)_{2}$ with def2-TZVP basis set for $4 \mathrm{f}^{\mathrm{n}}$ $5 \mathrm{~d}^{1}$ configurations and SCecp-mwb basis set for $4 \mathrm{f}^{\mathrm{n}+1}$ configurations.

\begin{tabular}{ccccc}
\hline Point Group & Term Symbol & Main Config. & Relative Energy $(e V)$ & Tb-Cp distance $(\AA)$ \\
\hline $\mathrm{C}_{1}$ & ${ }^{8} \mathrm{~A}$ & $4 \mathrm{f}^{8} 5 \mathrm{~d}^{1}$ & 0 & 2.520 \\
\hline $\mathrm{C}_{1}$ & ${ }^{6} \mathrm{~A}$ & $4 \mathrm{f}^{8} 5 \mathrm{~d}^{1}$ & 0.11 & 2.493 \\
\hline $\mathrm{C}_{1}$ & ${ }^{6} \mathrm{~A}$ & {$\left[4 \mathrm{f}^{9}\right]$} & - & 2.588 \\
\hline
\end{tabular}

Relative energy differences between ${ }^{8} \mathrm{~A}$ and ${ }^{6} \mathrm{~A}$ term symbols show similar preference for spin multiplicities in the $4 \mathrm{f}^{8} 5 \mathrm{~d}^{1}$ configuration. However, the difference is again small (on the order of $2 \mathrm{kcal} / \mathrm{mol}$ ), and within the accuracy range that DFT typically performs. Comparison of Tb-Cp distances between the $4 \mathrm{f}^{8} 5 \mathrm{~d}^{1}$ and $4 \mathrm{f}^{9}$ electronic configurations again support experimentally observed lengthening, although the computed change is closer to $0.1 \AA$. These results suggest adoption of the $4 \mathrm{f}^{9} 5 \mathrm{~d}^{1}$ configuration.

Table S21. Natural population analysis conducted for the ${ }^{8} \mathrm{~A}$ term symbol of $\mathrm{Tb}\left(\mathrm{Cp} \mathrm{p}^{\mathrm{iPr} 5}\right)_{2}$.

\begin{tabular}{ccccc}
\hline Atom & $\boldsymbol{n}(\boldsymbol{s})$ & $\boldsymbol{n}(\boldsymbol{p})$ & $\boldsymbol{n}\left(\boldsymbol{d z ^ { 2 }}\right)$ & $\boldsymbol{n}(\boldsymbol{d} \text { rest })^{*}$ \\
\hline $\mathrm{Tb}$ & 0.7261 & 0 & 0.1555 & 0 \\
\hline $\mathrm{C}^{* *}$ & 0.0073 & 0.0394 & 0 & 0 \\
\hline
\end{tabular}

* Includes natural occupancies from all other d orbitals

${ }^{* *}$ Represents summed contribution from the 10 Carbon atoms located on the two $\mathrm{Cp}$ rings.

Similar results are observed as from the population analysis as that for $\mathrm{Dy}\left(\mathrm{Cp}^{\mathrm{iPr} 5}\right)_{2}$, with the HOMO $\mathrm{s}$ orbital stabilization of the $5 \mathrm{~d} \mathrm{z}^{2}$ HOMO. P orbital contributions from the ligand serve to further stabilize the orbital relative to the other $5 \mathrm{~d}$ orbitals. While the ratio of $\mathrm{s}$ to $\mathrm{d}$ character is again uncharacteristically high, we attribute this to overestimation of s contributions in the natural population analysis method. 
Table S22. Comparison of significant electronic excitations (> 0.0015) from the first 55 for $\mathrm{C}_{1}$, term symbols computed with TDDFT using the TPSS functional. Blue shifted results are also included in parentheses. A double- $\zeta$ quality basis set (def2-SV(P)) was used for these response calculations due to reference instabilities with the triple- $\zeta$ quality basis.

\begin{tabular}{|c|c|c|c|c|c|}
\hline $\begin{array}{c}\text { Term } \\
\text { Symbol }\end{array}$ & $\begin{array}{l}\text { Wavelength } \\
\text { (nm) }\end{array}$ & $\begin{array}{l}\text { Oscillator } \\
\text { Str. }\end{array}$ & $\begin{array}{c}\text { Occ-virt orbital } \\
\text { transition }\end{array}$ & $\begin{array}{c}\text { Occupied } \\
\text { (virtual) } \\
\text { energy }(e V)\end{array}$ & $\begin{array}{c}\text { Type of } \\
\text { excitation }\end{array}$ \\
\hline \multirow[t]{16}{*}{${ }^{8} \mathrm{~A}$} & $450.2(426.9)$ & 0.00297 & $176 \mathrm{a} \rightarrow 178 \mathrm{a}$ & $-4.29(-1.48)$ & LMCT \\
\hline & $447.6(424.6)$ & 0.00243 & $175 \mathrm{a} \rightarrow 178 \mathrm{a}$ & $-4.31(-1.48)$ & LMCT \\
\hline & $430.5(409.2)$ & 0.01223 & $176 \mathrm{a} \rightarrow 179 \mathrm{a}$ & $-4.29(-1.38)$ & LMCT \\
\hline & $429.6(408.4)$ & 0.00886 & $175 \mathrm{a} \rightarrow 179 \mathrm{a}$ & $-4.31(-1.38)$ & LMCT \\
\hline & $420.8(400.4)$ & 0.00744 & $170 \mathrm{a} \rightarrow 189 \mathrm{a}$ & $-1.27(1.70)$ & MLCT \\
\hline & 418.5 (398.3) & 0.00175 & $170 \mathrm{a} \rightarrow 190 \mathrm{a}$ & $-1.27(1.70)$ & MLCT \\
\hline & 416.7 (396.7) & 0.00192 & $174 \mathrm{a} \rightarrow 178 \mathrm{a}$ & $-4.56(-1.48)$ & $4 f \rightarrow 5 d$ \\
\hline & $401.6(383.0)$ & 0.00314 & $174 \mathrm{a} \rightarrow 179 \mathrm{a}$ & $-4.56(-1.38)$ & $4 f \rightarrow 5 d$ \\
\hline & $371.0(355.1)$ & 0.00430 & $170 \mathrm{a} \rightarrow 196 \mathrm{a}$ & $-1.27(2.10)$ & $4 f \rightarrow 5 d$ \\
\hline & $349.6(335.4)$ & 0.00467 & $169 \mathrm{a} \rightarrow 171 \mathrm{a}$ & $-4.42(-0.82)$ & LMCT \\
\hline & $349.5(335.3)$ & 0.00442 & $168 \mathrm{a} \rightarrow 171 \mathrm{a}$ & $-4.42(-0.82)$ & LMCT \\
\hline & $329.3(316.6)$ & 0.00622 & $\begin{array}{c}169 \mathrm{a} \rightarrow 173 \mathrm{a} \\
(46 \%)\end{array}$ & $-4.42(-0.61)$ & LMCT \\
\hline & & & $\begin{array}{c}176 \mathrm{a} \rightarrow 180 \mathrm{a} \\
(30 \%)\end{array}$ & $-4.29(-0.44)$ & LMCT \\
\hline & $329.2(316.6)$ & 0.00768 & $168 \mathrm{a} \rightarrow 173 \mathrm{a}$ & $-4.42(-0.61)$ & LMCT \\
\hline & 320.7 (308.7) & 0.03444 & $176 \mathrm{a} \rightarrow 180 \mathrm{a}$ & $-4.29(-0.44)$ & LMCT \\
\hline & $319.4(307.5)$ & 0.03442 & $175 \mathrm{a} \rightarrow 180 \mathrm{a}$ & $-4.31(-0.44)$ & LMCT \\
\hline \multirow[t]{3}{*}{${ }^{6} \mathrm{~A} 4 \mathrm{f}^{\mathrm{n}} 5 \mathrm{~d}^{1}$} & 415.8 (395.9) & 0.00290 & $175 \mathrm{a} \rightarrow 177 \mathrm{a}$ & $-4.29(-1.25)$ & LMCT \\
\hline & $416.6(396.6)$ & 0.00291 & $176 \mathrm{a} \rightarrow 177 \mathrm{a}$ & $-4.29(-1.25)$ & LMCT \\
\hline & $370.1(354.2)$ & 0.00176 & $\begin{array}{c}169 \mathrm{a} \rightarrow 172 \mathrm{a} \\
(28 \%)\end{array}$ & $-4.44(-1.08)$ & LMCT \\
\hline
\end{tabular}




\begin{tabular}{|c|c|c|c|c|c|}
\hline & \multirow[b]{2}{*}{$370.0(354.1)$} & \multirow[b]{2}{*}{0.00174} & $\begin{array}{c}168 \mathrm{a} \rightarrow 172 \mathrm{a} \\
(19 \%) \\
168 \mathrm{a} \rightarrow 173 \mathrm{a} \\
(18 \%)\end{array}$ & $\begin{array}{l}-4.44(-1.08) \\
-4.44(-1.00)\end{array}$ & $\begin{array}{l}\text { LMCT } \\
\text { LMCT }\end{array}$ \\
\hline & & & $\begin{array}{c}168 \mathrm{a} \rightarrow 172 \mathrm{a} \\
(29 \%) \\
169 \mathrm{a} \rightarrow 172 \mathrm{a} \\
(19 \%) \\
169 \mathrm{a} \rightarrow 173 \mathrm{a} \\
(18 \%)\end{array}$ & $\begin{array}{l}-4.44(-1.08) \\
-4.44(-1.08) \\
-4.44(-1.00)\end{array}$ & $\begin{array}{l}\text { LMCT } \\
\text { LMCT } \\
\text { LMCT }\end{array}$ \\
\hline & $360.0(345.0)$ & 0.00774 & $167 \mathrm{a} \rightarrow 171 \mathrm{a}$ & $-4.85(-1.37)$ & LMCT \\
\hline & $360.0(345.0)$ & 0.00854 & $166 \mathrm{a} \rightarrow 171 \mathrm{a}$ & $-4.85(-1.36)$ & LMCT \\
\hline & $354.7(340.1)$ & 0.02083 & $173 \mathrm{a} \rightarrow 177 \mathrm{a}$ & $-4.86(-1.25)$ & LMCT \\
\hline & $354.5(340.0)$ & 0.01968 & $172 \mathrm{a} \rightarrow 177 \mathrm{a}$ & $-4.86(-1.25)$ & LMCT \\
\hline & $354.3(339.7)$ & 0.01770 & $173 \mathrm{a} \rightarrow 177 \mathrm{a}$ & $-4.86(-1.25)$ & LMCT \\
\hline & $354.1(339.6)$ & 0.00679 & $172 \mathrm{a} \rightarrow 177 \mathrm{a}$ & $-4.86(-1.25)$ & LMCT \\
\hline & $353.6(339.1)$ & 0.00524 & $174 \mathrm{a} \rightarrow 179 \mathrm{a}$ & $-4.62(-1.04)$ & $4 f \rightarrow 5 d$ \\
\hline & $340.1(326.7)$ & 0.00407 & $170 \mathrm{a} \rightarrow 178 \mathrm{a}$ & $-3.56(0.17)$ & $5 d \rightarrow 4 f$ \\
\hline & 320.9 (308.9) & 0.01209 & $170 \mathrm{a} \rightarrow 179 \mathrm{a}$ & $-3.56(0.40)$ & $5 d \rightarrow 4 f$ \\
\hline & 317.7 (305.9) & 0.00976 & $170 \mathrm{a} \rightarrow 180 \mathrm{a}$ & $-3.56(0.44)$ & $5 d \rightarrow 4 f$ \\
\hline & $291.3(281.4)$ & 0.01350 & $170 \mathrm{a} \rightarrow 181 \mathrm{a}$ & $\begin{array}{l}-3.56(0.73) \\
-3.56(0.74)\end{array}$ & $\begin{array}{l}\text { MLCT } \\
\text { MLCT }\end{array}$ \\
\hline & $291.0(281.1)$ & 0.01358 & $170 \mathrm{a} \rightarrow 182 \mathrm{a}$ & $-4.44(-0.09)$ & LMCT \\
\hline & $286.6(277.0)$ & 0.00885 & $169 \mathrm{a} \rightarrow 177 \mathrm{a}$ & $-4.44(-0.09)$ & LMCT \\
\hline & $286.5(276.4)$ & 0.00891 & $168 \mathrm{a} \rightarrow 177 \mathrm{a}$ & $-5.71(-1.25)$ & $4 f \rightarrow 5 d$ \\
\hline & $282.9(273.5)$ & 0.00301 & $170 \mathrm{a} \rightarrow 177 \mathrm{a}$ & & \\
\hline${ }^{6} \mathrm{~A} 4 \mathrm{f}^{\mathrm{n}+1}$ & $386.6(369.3)$ & 0.0070 & $160 \mathrm{a} \rightarrow 161 \mathrm{a}$ & $-4.25(-1.03)$ & LMCT \\
\hline & $320.3(308.4)$ & 0.0602 & $160 \mathrm{a} \rightarrow 163 \mathrm{a}$ & $-4.25(-0.52)$ & LMCT \\
\hline
\end{tabular}




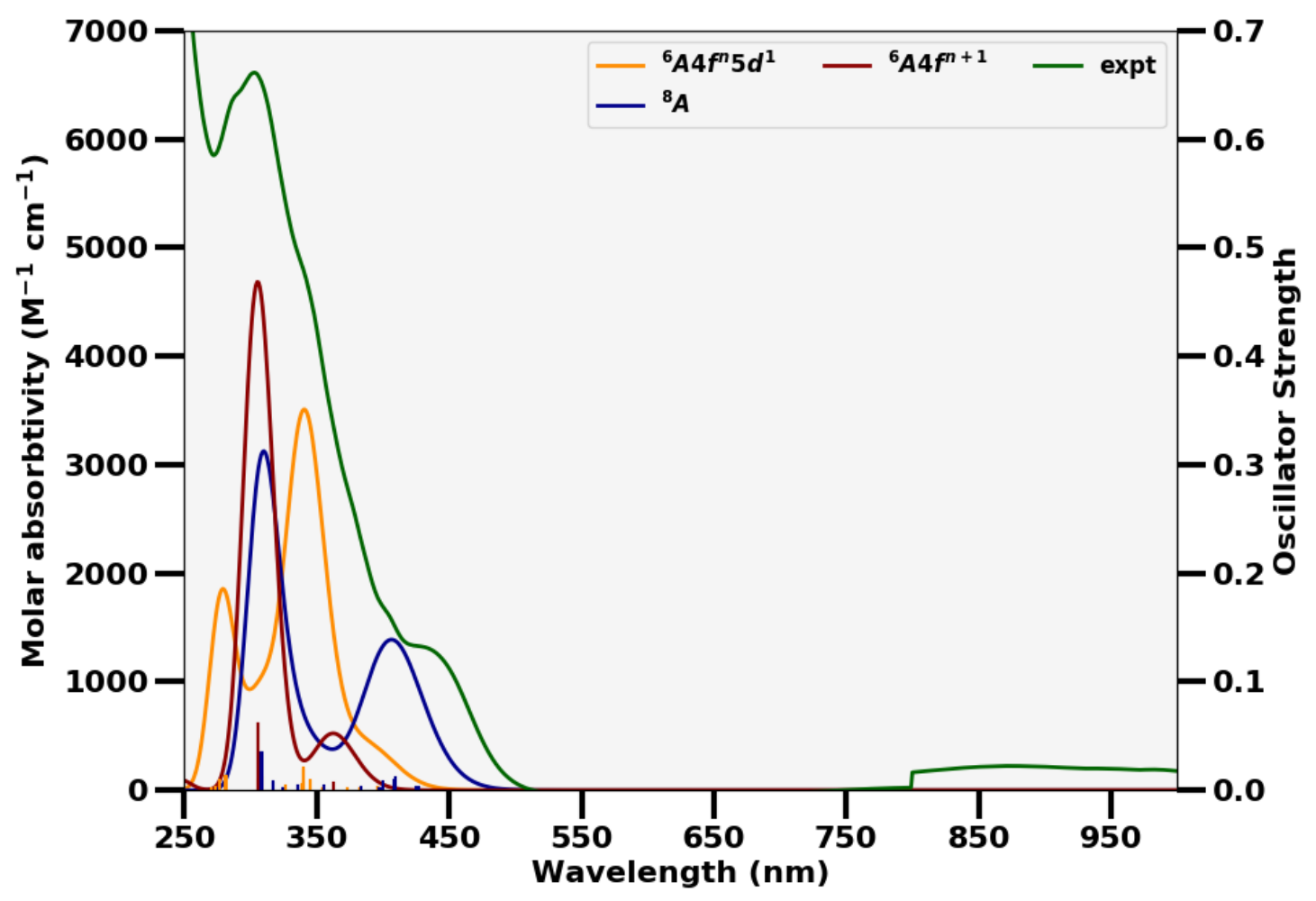

Figure S85. Computed UV-Vis spectra for $\mathrm{Tb}\left(\mathrm{Cp}^{\mathrm{iPr} 5}\right)_{2}$ with corresponding term symbols in the $\mathrm{C}_{1}$ point group. A RMS width of $0.15 \mathrm{eV}$, a blue shift of $0.15 \mathrm{eV}$, and a vertical scaling of $0.5 \mathrm{eV}$ was used. Details for each individual excitation can be found in Table S22.

Due to instabilities in the TD-DFT routine, electronic excitations were unable to be computed with a triple- $\zeta$ quality basis set on the metal atom, so the def2-SV(P) basis was used. The obtained experimental UV-Vis spectrum is similar to that of $\mathrm{Dy}\left(\mathrm{Cp}^{\mathrm{iPr} 5}\right)_{2}$, with two primary peaks predicted to correspond to ligand-metal charge transfer excitations. The spectra predicted with DFT for the ${ }^{8} \mathrm{~A}$ and ${ }^{6} \mathrm{~A}$ terms also correspond well with the Dy case despite being limited to a smaller basis. The ${ }^{6} \mathrm{~A} 4 \mathrm{f}^{\mathrm{n}} 5 \mathrm{~d}^{1}$ spectrum is again red-shifted from the other two predicted spectra. The ligand-metal excitation is however much weaker than that of the others. There is also an absence of metal-metal excitations with longer wavelengths, as seen in the $\mathrm{Dy}\left(\mathrm{Cp} \mathrm{p}^{\mathrm{iPr} 5}\right)_{2}$ case. This is likely due to the smaller basis set used for the lanthanide atom. The ${ }^{8} \mathrm{~A} 4 \mathrm{f}^{\mathrm{n}} 5 \mathrm{~d}^{1}$ again qualitatively matches the experimental spectrum better than that of the $4 \mathrm{f}^{\mathrm{n}+1}$ configuration. 
Table S23. Computed metal-centered $4 \mathrm{f}$ and $5 \mathrm{~d}$ molecular orbitals and energies (in $\mathrm{eV}$ ) for term symbol ${ }^{8} \mathrm{~A}$ with def2-TZVP basis. The other $4 \mathrm{f}^{\mathrm{n}} 5 \mathrm{~d}^{1}$ term yields nearly identical MOs. Note that orbital numbering does not necessarily correspond to other term symbols.

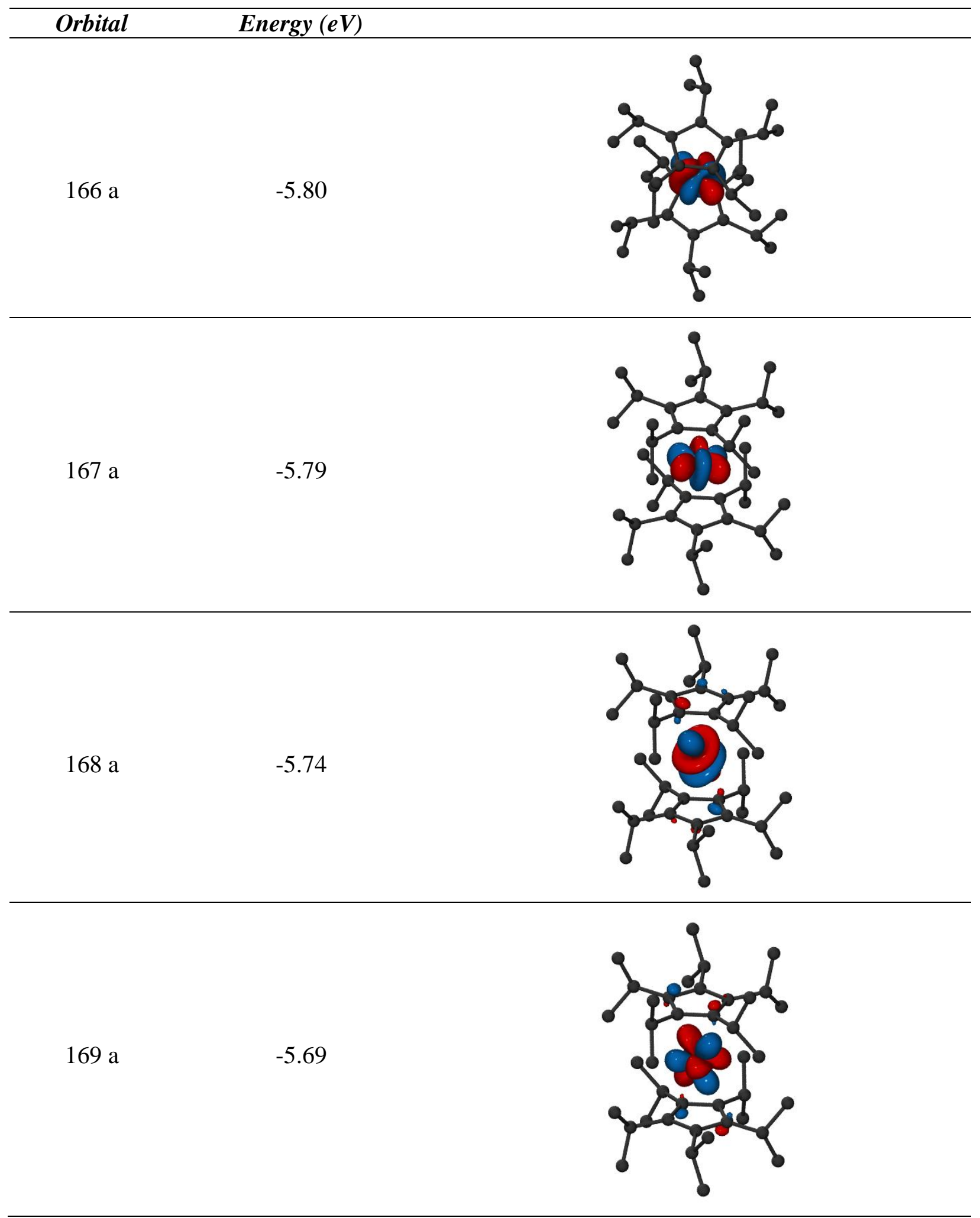




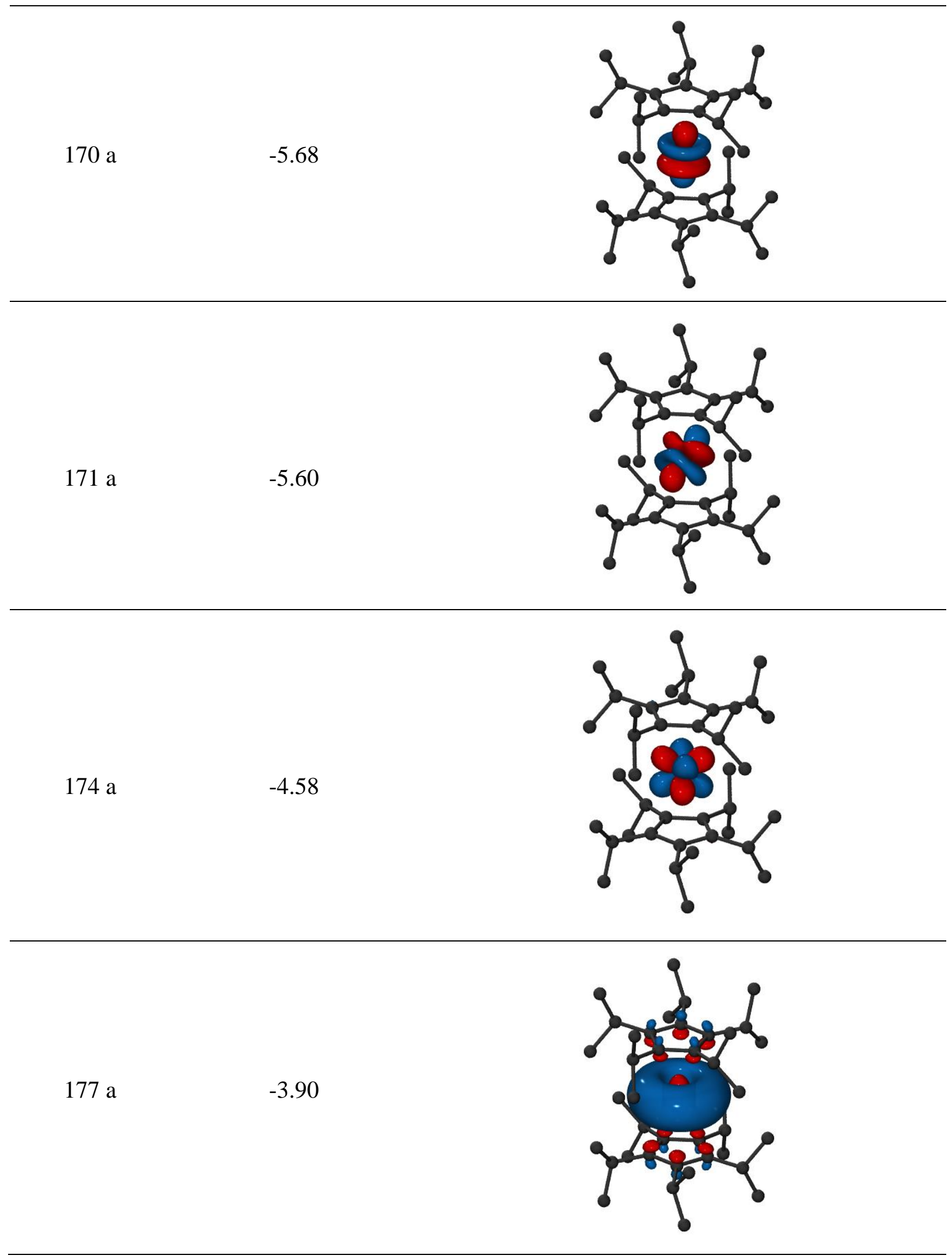




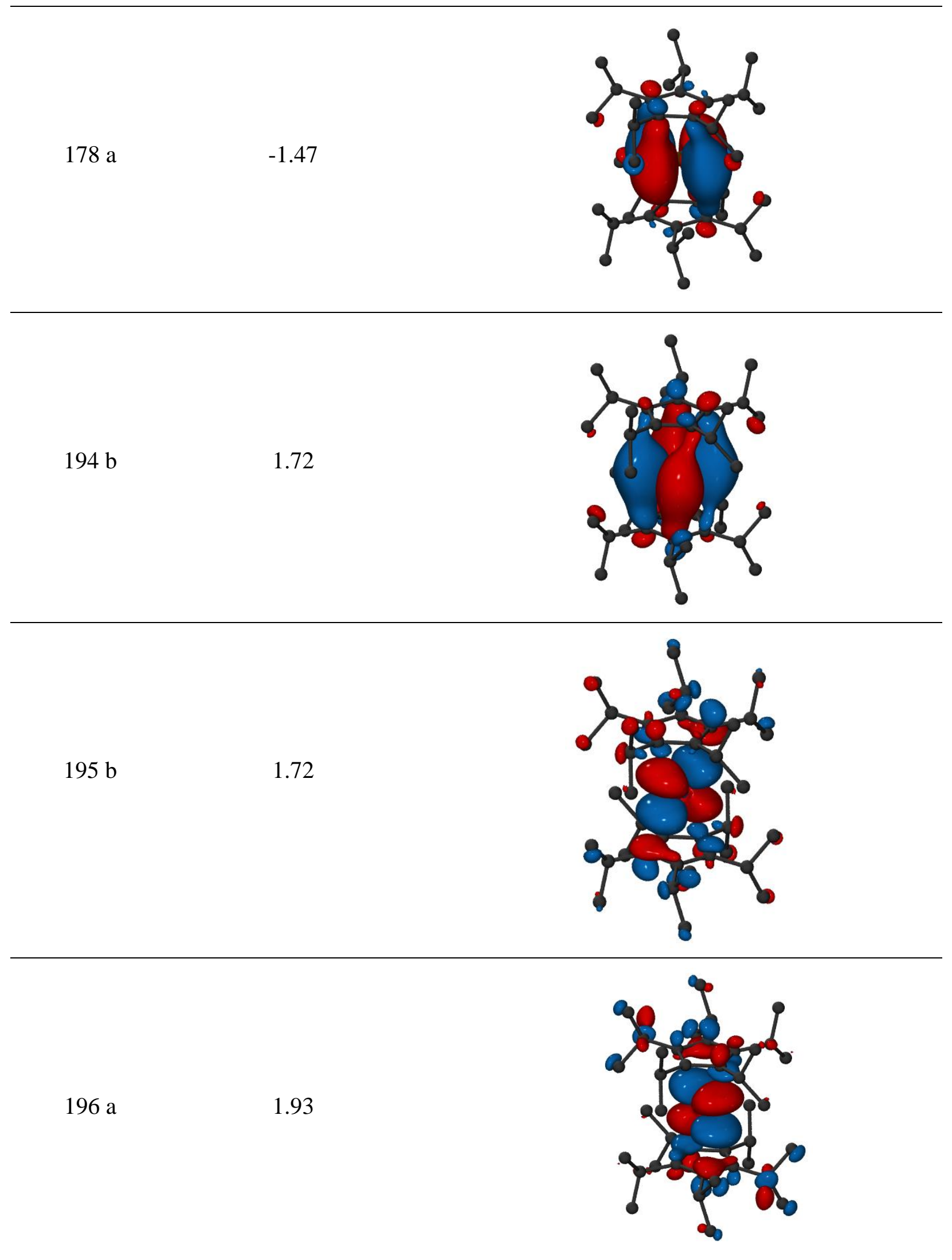




\section{References}

(1) Connelly, S. J.; Kaminsky, W.; Heinekey, D. M. Structure and Solution Reactivity of (Triethylsilylium)triethylsilane Cations. Organometallics 2013, 32, 7478.

(2) McClain, K. R.; Gould, C. A.; Chakarawet, K.; Teat, S. J.; Groshens, T. J.; Long, J. R.; Harvey, B. G. High-temperature magnetic blocking and magneto-structural correlations in a series of dysprosium(III) metallocenium single-molecule magnets. Chem. Sci. 2018, 9, 84928503.

(3) APEX3, v. 2009; Bruker Analytical X-Ray Systems, Inc: Madison, WI, 2009.

(4) SAINT and APEX 2 Software for CCD Diffractometers, Bruker Analytical X-ray Systems Inc., Madison, WI, USA, 2014.

(5) Rigaku Oxford Diffraction, (2015), CrysAlisPro Software system, version 1.171.39.7a, Rigaku Corporation, Oxford, UK.

(6) Sheldrick, G. M. SHELXT, University of Göttingen, Germany, 2015

(7) Sheldrick, G. M. SHELXL, University of Göttingen, Germany, 2015

(8) Staroverov, V. N.; Scuseria, G.E.; Tao, J.; Perdew, J. P. Comparative assessment of a new nonempirical density functional: Molecules and hydrogen-bonded complexes. J. Chem Phys. 2003, 119, 12129-121237

(9) Neese, F.; Olbrich, G. Efficient use of the resolution of the identity approximation in timedependent density functional calculations with hybrid density functionals. Chem. Phys. Lett. 2002, 362(1-2), 170-178.

(10) Grimme, S.; Antony, J.; Ehrlich, S.; Krieg, H. A consistent and accurate ab initio parametrization of density functional dispersion correction (DFT-D) for the 94 elements H-Pu. $J$. Chem. Phys. 2010, 132(15), 154104.

(11) Deglmann, P.; Furche, F. Efficient characterization of stationary points on potential energy surfaces. J. Chem. Phys. 2002, 117(21), 9535-9538.

(12) Weigend, F.; Ahlrichs, R. Balanced basis sets of split valence, triple zeta valence and quadruple zeta valence quality for $\mathrm{H}$ to $\mathrm{Rn}$ : Design and assessment of accuracy. Phys. Chem. Chem. Phys. 2005, 7(18), 3297-3305

(13) Gulde, R. ; Pollak, P. ; Weigend F. Error-balanced segmented contracted basis sets of Double$\zeta$ to Quadruple- $\zeta$ valence quality for lanthanides. J. Chem. Theory Comput. 2012, 8 , 11, 40624068

(14) Dolg, M.; Stoll, H.; Preuss, H. Energy-adjusted ab initio pseudopotentials for rare earth elements. J. Chem. Phys. 1989, 90, $1730-1734$.

(15) Cao, X.; \& Dolg, M. Valence basis sets for relativistic energy-consistent small-core lanthanide pseudopotentials. J. Chem. Phys. 2001, 115(16), 7348-7355.

(16) Hülsen, M.; Weigand, A.; Dolg, M. Quasirelativistic energy-consistent 4f-in-core pseudopotentials for tetravalent lanthanide elements. Theo. Chem. Acc. 2009, 122(1-2), 23-29.

(17) Furche, F.; Ahlrichs, R. Adiabatic time-dependent density functional methods for excited state properties. J. Chem. Phys. (2002), 117(16), 7433-7447.

(18) Bauernschmitt, R.; Ahlrichs, R. Treatment of electronic excitations within the adiabatic approximation of time dependent density functional theory. Chem. Phys. Lett. 1996, 256, 454-464. (19) Bates J.; Furche F. Harnessing the meta-generalized gradient approximation for timedependent density functional theory. J. Chem. Phys. 2012, 137, 164105/1-164105/10.

(20) Humphrey, W.; Dalke, A.; Schulten, K. VMD: visual molecular dynamics. J. Mol. Graph. 1996, 14(1), 33-38. 
(21) Furche, F.; Ahlrichs, R.; Hättig, C.; Klopper, W.; Sierka, M.; Weigend, F. Turbomole. Wiley Interdiscip. Rev. Comput. Mol. Sci. 2014, 4(2), 91-100

(22) Fieser, M. E.; MacDonald, M. R.; Krull, B. T.; Bates, J. E.; Ziller, J. W.; Furche, F.; Evans, W. J. Structural, spectroscopic, and theoretical comparison of traditional vs recently discovered $\mathrm{Ln}^{2+}$ ions in the $[\mathrm{K}(2.2 .2$-cryptand $)]\left[\left(\mathrm{C}_{5} \mathrm{H}_{4} \mathrm{SiMe}_{3}\right)_{3} \mathrm{Ln}\right]$ complexes: the variable nature of $\mathrm{Dy}^{2+}$ and $\mathrm{Nd}^{2+}$. J. Am. Chem. Soc. 2014, 137(1), 369-382.

(23) Reed, A. E.; Weinstock, R. B.; Weinhold, F. Natural population analysis. J. Chem. Phys. 1985, 83(2), 735-746.

(24) Fronzoni, G.; Colavita, P.; Stener, M.; De Alti, G.; Decleva, P. Theoretical study of photoionization processes in $\mathrm{Fe}\left(\mathrm{C}_{5} \mathrm{H}_{5}\right)_{2}$. J. Phys. Chem. A 2001, 105(42), 9800-9812.

(25) Bean, D. E.; Fowler, P. W.;Morris, M. J. Aromaticity and ring currents in ferrocene and two isomeric sandwich complexes. J. Organomet. Chem. 2011, 696(10), 2093-2100. 